\title{
\#USGS
}

A Conceptual Model for Site-Level Ecology of the Giant Gartersnake (Thamnophis gigas) in the Sacramento Valley, California

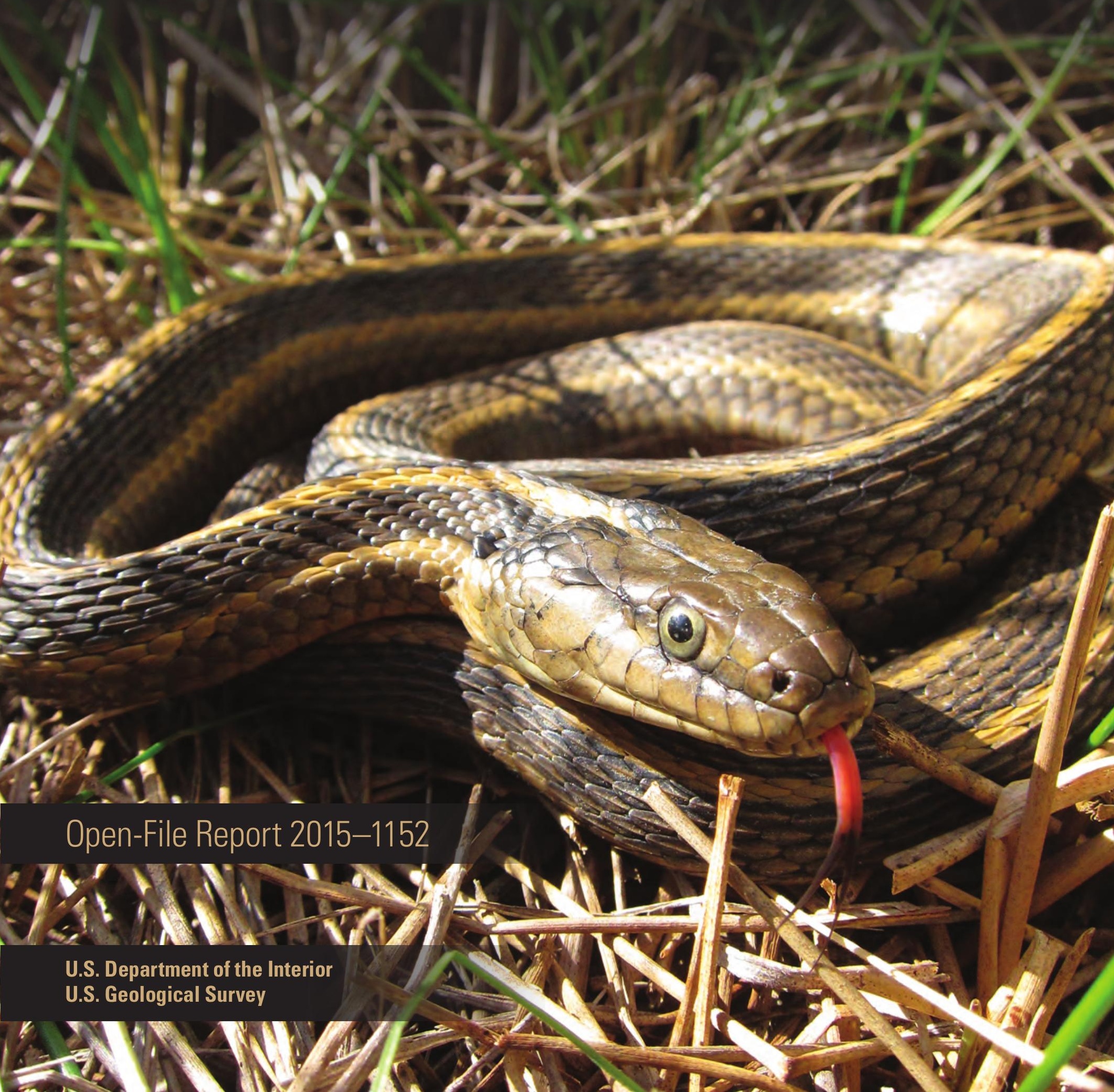


Cover: An adult giant gartersnake (Thamnophis gigas). Photograph by Matt Meshriy, U.S. Geological Survey, 2011. 


\section{A Conceptual Model for Site-Level Ecology of the Giant Gartersnake (Thamnophis gigas) in the Sacramento Valley, California}

By Brian J. Halstead, Glenn D. Wylie, Michael L. Casazza, Eric C. Hansen, Rick D. Scherer, and Laura C. Patterson

Prepared in cooperation with the California Department of Water Resources

Open-File Report 2015-1152

U.S. Department of the Interior

U.S. Geological Survey 


\section{U.S. Department of the Interior \\ SALLY JEWELL, Secretary}

\section{U.S. Geological Survey \\ Suzette M. Kimball, Acting Director}

U.S. Geological Survey, Reston, Virginia: 2015

For more information on the USGS—-the Federal source for science about the Earth,

its natural and living resources, natural hazards, and the environment-visit

http://www.usgs.gov or call 1-888-ASK-USGS

For an overview of USGS information products, including maps, imagery, and publications, visit http://www.usgs.gov/pubprod

Any use of trade, product, or firm names is for descriptive purposes only and does not imply endorsement by the U.S. Government.

Although this information product, for the most part, is in the public domain, it also may contain copyrighted materials as noted in the text. Permission to reproduce copyrighted items must be secured from the copyright owner.

Halstead, B.J., Wylie, G.D., Casazza, M.L., Hansen, E.C., Scherer, R.D., and Patterson, L.C., 2015, A conceptual model for site-level ecology of the giant gartersnake (Thamnophis gigas) in the Sacramento Valley, California: U.S. Geological Survey Open-File Report 2015-1152, 152 p., http://dx.doi.org/10.3133/ofr20151152.

ISSN 2331-1258 (online) 


\section{Contents}

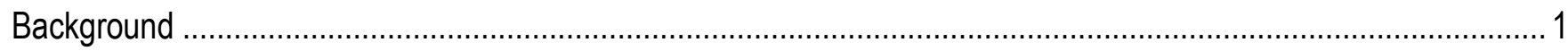

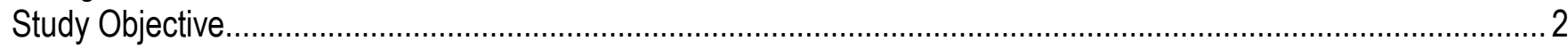

Methods

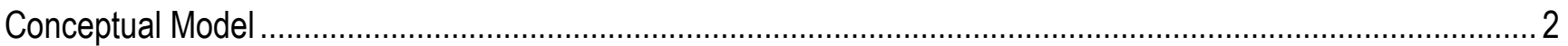

Populating Conditional Probability Tables .............................................................................................. 4

Model Review and Revision .........................................................................................................

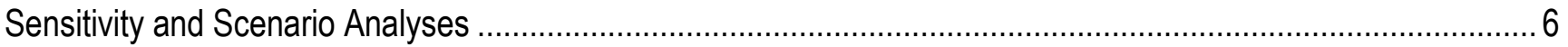

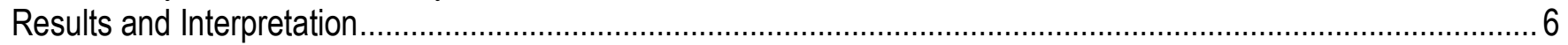

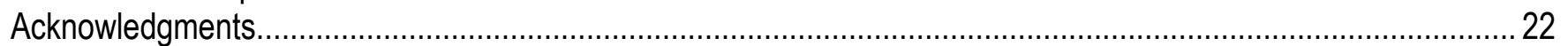

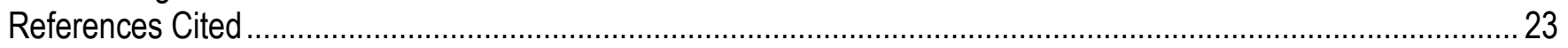

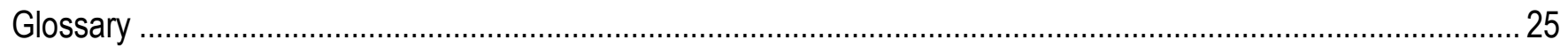

Appendix A. Narrative Description of Nodes, and Logic and Assumptions Underlying Conditional

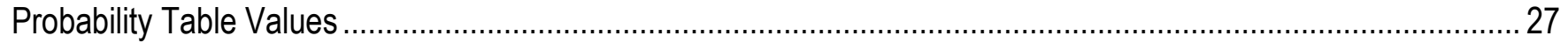

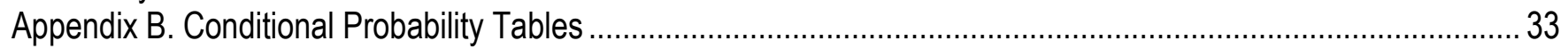

\section{Figures}

Figure 1. Version 0.10a of the Bayesian network model for the giant gartersnake (Thamnophis gigas) in the Sacramento Valley, California.

Figure 2. Version 1.00b of the Bayesian network model for the giant gartersnake (Thamnophis gigas), in the Sacramento Valley, California.

Figure 3. Most likely states of all nodes in Bayesian network model given a finding of increasing population growth of giant gartersnakes (Thamnophis gigas) in the Sacramento Valley, California .....

Figure 4. Most likely states of all nodes in Bayesian network model given a finding of decreasing population growth of giant gartersnakes (Thamnophis gigas) in the Sacramento Valley, California

Figure 5. Most likely outcomes of the Bayesian network model for the giant gartersnake (Thamnophis gigas)

in the Sacramento Valley, California, with ephemeral water-management conditions.

Figure 6. Most likely outcomes of the Bayesian network model for the giant gartersnake (Thamnophis gigas) in the Sacramento Valley, California, with winter water-management conditions typical for wintering waterfowl management.

Figure 7. Most likely outcomes of the Bayesian network model for the giant gartersnake (Thamnophis gigas) in the Sacramento Valley, California, with groundwater substitution water-management conditions (assuming groundwater applied during the summer as for summer water management).

Figure 8. Most likely outcomes of the Bayesian network model for the giant gartersnake (Thamnophis gigas) in the Sacramento Valley, California, with summer water-management conditions (that is, water only available during the summer as typical of rice agriculture)

Figure 9. Most likely outcomes of the Bayesian network model for the giant gartersnake (Thamnophis gigas) in the Sacramento Valley, California, with permanent water-management conditions (that is, water in marshes all year)......

Figure 10. Best-case scenario in Bayesian network model for increasing population growth rate of giant gartersnake (Thamnophis gigas), in the Sacramento Valley, California..

Figure 11. Worst-case scenario in Bayesian network model for decreasing population growth rate of giant gartersnake (Thamnophis gigas), in the Sacramento Valley, California.. 


\section{Tables}

Table 1. Sensitivity analysis diagnostics applied to the Bayesian network model for population growth of giant gartersnakes (Thamnophis gigas) in the Sacramento Valley, California, with uninformative (uniform) priors on all parent nodes

Table 2. Sensitivity analysis diagnostics applied to the Bayesian network model for adult survival of giant gartersnakes (Thamnophis gigas) in the Sacramento Valley, California, with uninformative (uniform) priors on all parent nodes

Table 3. Sensitivity analysis diagnostics applied to the Bayesian network model for first-year survival of giant gartersnakes (Thamnophis gigas) in the Sacramento Valley, California, with uninformative (uniform) priors on all parent nodes

Table 4. Sensitivity analysis diagnostics applied to the Bayesian network model for fecundity of giant gartersnakes (Thamnophis gigas) in the Sacramento Valley, California, with uninformative (uniform) priors on all parent nodes

Table 5. Sensitivity analysis diagnostics applied to the Bayesian network model for habitat quality of giant gartersnakes (Thamnophis gigas) in the Sacramento Valley, California, with uninformative (uniform) priors on all parent nodes 


\section{A Conceptual Model for Site-Level Ecology of the Giant Gartersnake (Thamnophis gigas) in the Sacramento Valley, California}

By Brian J. Halstead ${ }^{1}$, Glenn D. Wylie'1, Michael L. Casazza1', Eric C. Hansen², Rick D. Scherer ${ }^{3}$, and Laura C. Patterson 4

\section{Background}

Giant gartersnakes (Thamnophis gigas) comprise a species of semi-aquatic snakes precinctive to marshes in the Central Valley of California (Hansen and Brode, 1980; Rossman and others, 1996). Because more than 90 percent of their historical wetland habitat has been converted to other uses (Frayer and others, 1989; Garone, 2007), giant gartersnakes have been listed as threatened by the State of California (California Department of Fish and Game Commission, 1971) and the United States (U.S. Fish and Wildlife Service, 1993). Giant gartersnakes currently occur in a highly modified landscape, with most extant populations occurring in the rice-growing regions of the Sacramento Valley, especially near areas that historically were tule marsh habitat (Halstead and others, 2010, 2014).

In ricelands and managed marshes, many operational decisions likely affect the health and viability of giant gartersnake populations. Land-use decisions, including the management of water, aquatic vegetation, terrestrial vegetation, and co-occurring species, have the potential to affect giant gartersnakes. Little is known, however, about the effects of these types of decisions on the viability of giant gartersnake populations.

Bayesian network models are a useful tool to help guide decisions with uncertain outcomes. These models require the articulation of what experts think they know about a system, and facilitate learning about the hypothesized relations (Marcot and others, 2001; Uusitalo, 2007). Bayesian networks further provide a clear visual display of the model that facilitates understanding among various stakeholders (Marcot and others, 2001; Uusitalo, 2007). Empirical data and expert judgment can be combined, as continuous or categorical variables, to update knowledge about the system (Marcot and others, 2001; Uusitalo, 2007). Importantly, Bayesian network models allow inference from causes to consequences, but also from consequences to

\footnotetext{
${ }^{1}$ U.S. Geological Survey.

${ }^{2}$ Eric C. Hansen Consulting.

${ }^{3}$ Department of Fish, Wildlife, and Conservation Biology, Colorado State University.

${ }^{4}$ California Department of Fish and Wildlife.
} 
causes, so that data can inform the states of nodes (values of different random variables) in either direction (Marcot and others, 2001; Uusitalo, 2007). Because they can incorporate both decision nodes that represent management actions and utility nodes that quantify the costs and benefits of outcomes, Bayesian networks are ideally suited to risk analysis and adaptive management (Nyberg and others, 2006; Howes and others, 2010). Thus, Bayesian network models are useful in situations where empirical data are not available, such as questions concerning the responses of giant gartersnakes to management.

\section{Study Objective}

The objective of this project was to develop a conceptual model of site-specific ecology of the giant gartersnake in the Sacramento Valley of California. We chose to develop the model at a site-specific scale because that is the scale at which most management decisions are made and at which giant gartersnake responses can be quantified. Because we used a Bayesian network model, we also quantified uncertainty associated with different nodes affecting ecology of the giant gartersnake, and the strength of influence of different variables on population growth rates of giant gartersnakes. We view this as a preliminary step in an ongoing process to clarify and quantify the effects of management actions on giant gartersnake populations.

\section{Methods}

\section{Conceptual Model}

The first step in developing our Bayesian network model was to construct a conceptual model for ecology of the giant gartersnake at the site level for populations in the Sacramento Valley of California. We chose the site level because it focused on variables that could be determined through measurements in the field and manipulated by resource managers. We attempted to follow structural guidelines of Marcot and others (2006) to the extent possiblenamely, keeping the number of parent nodes (variables that exert an influence on the variable of interest) to three or fewer, keeping the number of states (different values each variable can take) per node to five or fewer, and having no more than four levels in the model. Exceptions to these guidelines were that we used five parent nodes for habitat quality and four for prey availability; these exceptions were believed to be necessary to represent the complexity of suitable habitat and factors affecting prey availability for giant gartersnakes (fig. 1). Most nodes without parents (hereafter referred to as "parentless nodes;" variables that are not influenced by other variables in the model) were selected to be measurable in the field at a site. Exceptions to this guideline were competitor effects, which likely are complex for giant gartersnakes (for example, much intraguild predation [predators preying upon each other] occurs, and the community of competitors likely varies with snake size), and winter refuge availability, which is difficult to quantify in the field (fig. 1). This version of the model (Version 0.10a following the conventions of Marcot and others [2001]), was used as a starting point for initiating discussion and expert review. We used Netica ${ }^{\mathrm{TM}} 5.12$ (Norsys Software Corporation, Vancouver, British Columbia, Canada) to construct and analyze the Bayesian network models. 


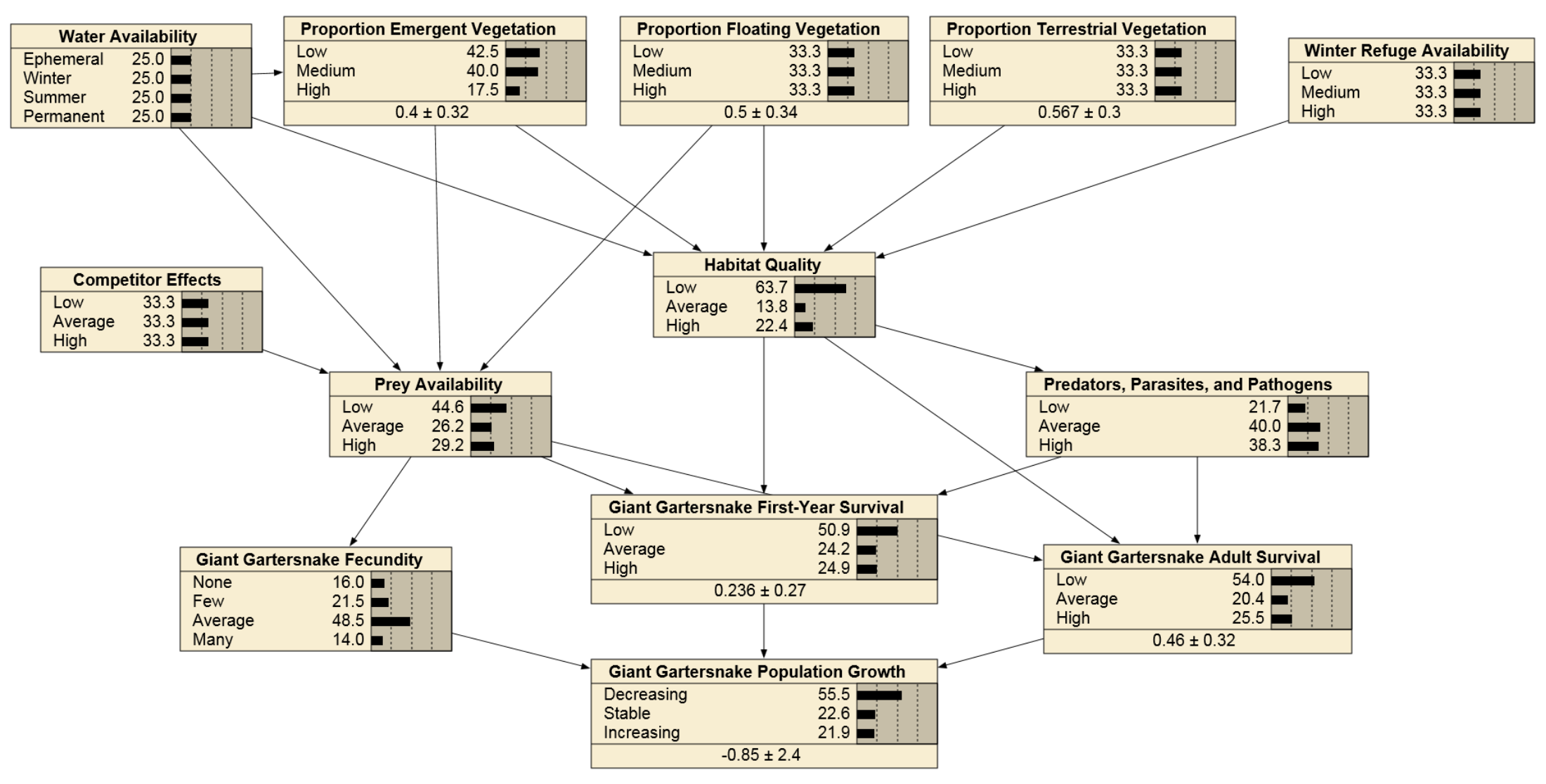

Figure 1. Version 0.10a of the Bayesian network model for the giant gartersnake (Thamnophis gigas) in the Sacramento Valley, California. The model is shown here with uniform priors on all parentless nodes. 


\section{Populating Conditional Probability Tables}

Conditional probability tables (CPTs) govern the relations between parent nodes and their children (Marcot and others, 2001; Nyberg and others, 2006; Uusitalo, 2007). Because few data on the relations among nodes in the conceptual model exist, we largely relied on in-house expert opinion to populate CPTs. Where data existed in the literature, we attempted to include these effects in the CPTs. Whenever possible, we used data from the literature or personal experience to discretize continuous nodes into meaningful categories (appendix A). Conditional probability tables are presented in appendix B.

\section{Model Review and Revision}

We submitted Version 0.10a of the Bayesian network model for the giant gartersnakeincluding model structure, CPTs, and a narrative description of the definitions of nodes and assumptions about relations indicated in the CPTs - to a technical advisory committee of giant gartersnake experts from regulatory agencies and consultants assembled by the California Department of Water Resources. This committee reviewed the structure and logic of the models, and suggested the inclusion of additional nodes and states, as well as the modification of some relations. Changes to Version 0.10a included (1) the addition of several nodes (water quality, submerged vegetation, and other sources of mortality), (2) the addition of another state to the water availability node (groundwater), and (3) strengthening the influence of predation on firstyear survival (see appendixes A and B). This revised model (Version 1.00b; fig. 2) then was analyzed for sensitivity of nodes of interest (demographic rates and habitat quality) to the values of other nodes and to examine most likely scenarios for positive and negative outcomes for giant gartersnake populations. 


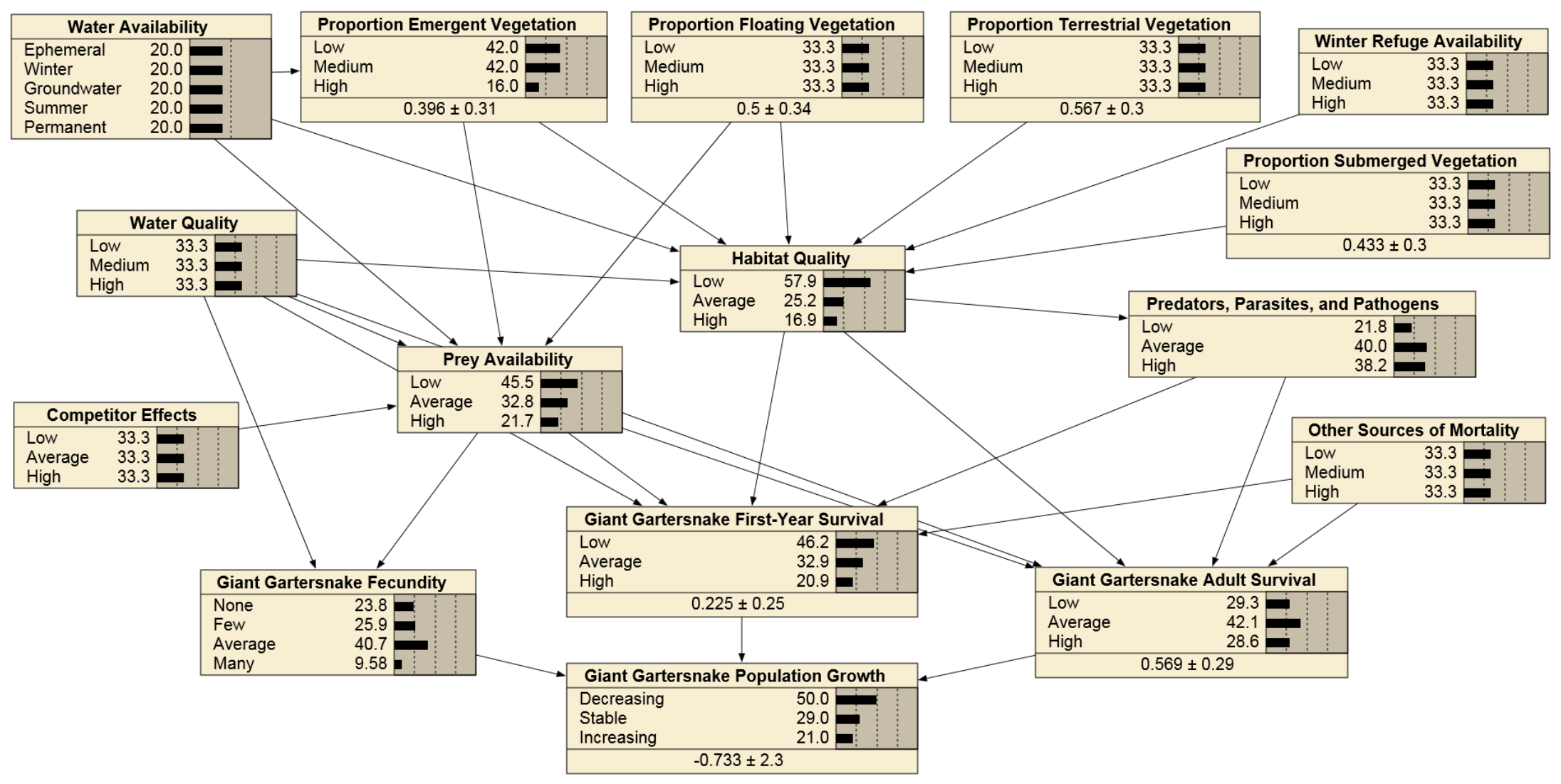

Figure 2. Version 1.00b of the Bayesian network model for the giant gartersnake (Thamnophis gigas), in the Sacramento Valley, California. This version of the model was subjected to sensitivity and scenario analyses. The model is shown here with uniform priors on all parentless nodes. 


\section{Sensitivity and Scenario Analyses}

We used two built-in sensitivity analyses provided by Netica ${ }^{\mathrm{TM}}$ to examine the influence of each node on several nodes of interest: variance reduction and mutual information (also referred to as “entropy reduction”). Variance reduction examines the reduction in the uncertainty of a node, given that the value of another node is known; the greater the variance reduction, the more the nodes affect each other. Mutual information is a measure of the amount of information shared by two nodes; like variance reduction, the greater the mutual information contained in two nodes, the stronger their effects on one another. We were particularly interested in how findings at each of the nodes in the network affected beliefs about the nodes for demographic parameters (population growth rate, adult survival, first-year survival, and fecundity) and habitat quality. For all sensitivity analyses, we assumed uniform (uninformative) priors on all parentless nodes. We also used scenario analysis for several scenarios. In two scenarios, we examined the most likely values of parent nodes that produced decreasing or increasing populations of giant gartersnakes. In five scenarios, we examined the influence of different water-management scenarios (that is, ephemeral [water available only briefly, regardless of season], winter water, groundwater, summer water [May through August, as typically applied to rice fields], or permanent water) on giant gartersnake population growth while maintaining uniform (uninformative) priors on all other parentless nodes. Finally, we adjusted all parentless nodes to maximize the likelihood of increasing or decreasing giant gartersnake population growth in two additional scenarios.

\section{Results and Interpretation}

The scenario and sensitivity analysis provided several insights into giant gartersnake population growth. When we set population growth to increasing, the most likely states for other demographic parameters were high adult and first-year survival and average fecundity (fig. 3). States within most other nodes remained relatively uncertain, except that high prevalence of predators, parasites, and pathogens was unlikely; medium emergent vegetation was likely; and water availability was likely to be summer or permanent. Water quality also was likely to be high (fig. 3). In contrast, when we set population growth to decreasing, adult and first-year survival were likely to be low, and no recruitment of neonates was the most likely finding for fecundity (fig. 4). Habitat quality, prey availability, and proportion emergent vegetation were all likely to be low, but the prevalence of predators, parasites, and pathogens was likely to be high (fig. 4). The sensitivity analysis for the population growth of giant gartersnakes indicated that adult survival had the largest influence on population growth, followed by fecundity, first-year survival, and habitat quality (table 1). Of the remaining variables, only prey availability; predators, parasites, and pathogens; and water availability had appreciable influence on giant gartersnake population growth, although water quality and proportion emergent vegetation had much more influence than the remaining nodes (table 1). 


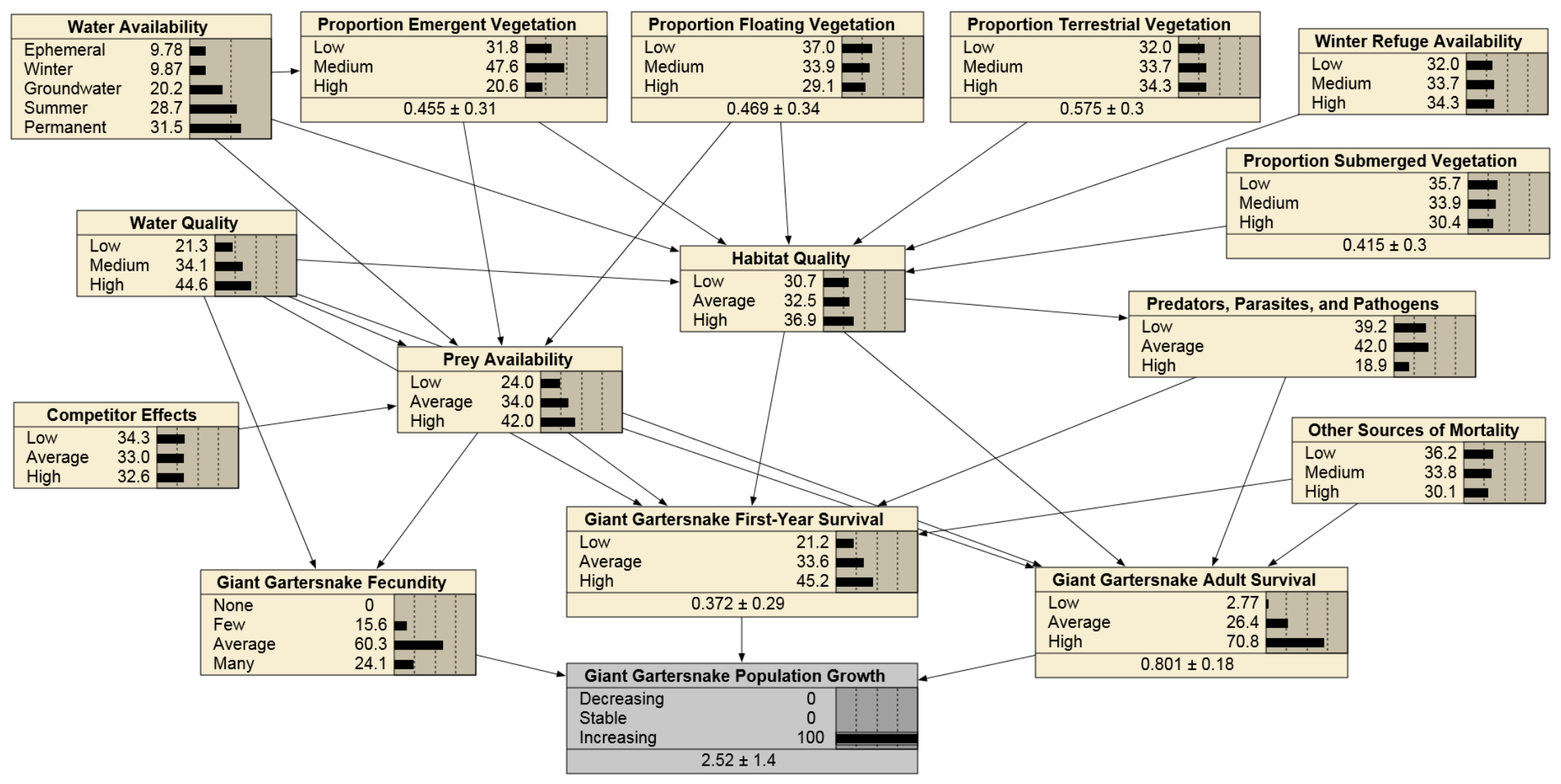

Figure 3. Most likely states of all nodes in Bayesian network model given a finding of increasing population growth of giant gartersnakes (Thamnophis gigas) in the Sacramento Valley, California. All parentless nodes were given uninformative (uniform) priors. Values in gray nodes were set at a fixed value to examine their influence on the remaining nodes in the network. 


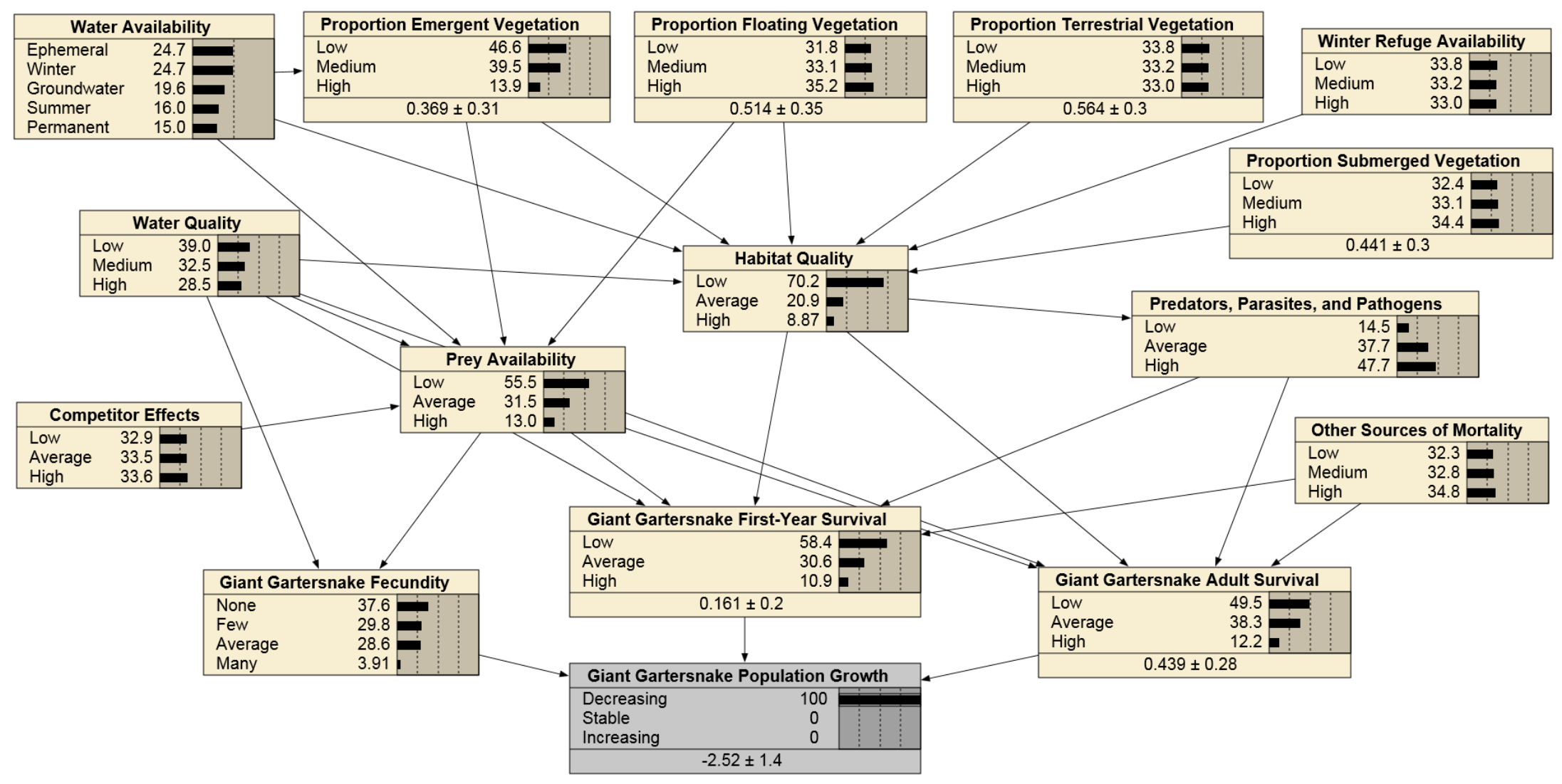

Figure 4. Most likely states of all nodes in Bayesian network model given a finding of decreasing population growth of giant gartersnakes (Thamnophis gigas) in the Sacramento Valley, California. All parentless nodes were given uninformative (uniform) priors. Values in gray nodes were set at a fixed value to examine their influence on the remaining nodes in the network. 
Table 1. Sensitivity analysis diagnostics applied to the Bayesian network model for population growth of giant gartersnakes (Thamnophis gigas) in the Sacramento Valley, California, with uninformative (uniform) priors on all parent nodes.

[Nodes are listed in order of decreasing influence on population growth. For all measures, greater values represent greater influence on population growth. Population growth is included for reference, as it represents the maximum variance reduction (VR) and mutual information (MI). <, less than]

\begin{tabular}{|c|c|c|c|c|c|}
\hline Node & $\begin{array}{l}\text { Variance } \\
\text { reduction }\end{array}$ & Percent VR & $\begin{array}{c}\text { Mutual } \\
\text { information }\end{array}$ & Percent MI & $\begin{array}{c}\text { Variance of } \\
\text { beliefs }\end{array}$ \\
\hline $\begin{array}{l}\text { Giant gartersnake population } \\
\text { growth }\end{array}$ & 5.435 & 100.00 & 1.491 & 100.00 & 0.402 \\
\hline Giant gartersnake adult survival & 1.184 & 21.80 & 0.260 & 17.40 & 0.053 \\
\hline Giant gartersnake fecundity & 0.812 & 14.90 & 0.178 & 11.90 & 0.029 \\
\hline $\begin{array}{l}\text { Giant gartersnake first-year } \\
\text { survival }\end{array}$ & 0.491 & 9.04 & 0.091 & 6.11 & 0.015 \\
\hline Habitat quality & 0.442 & 8.14 & 0.082 & 5.48 & 0.013 \\
\hline Prey availability & 0.347 & 6.38 & 0.064 & 4.28 & 0.010 \\
\hline $\begin{array}{l}\text { Predators, parasites, and } \\
\text { pathogens }\end{array}$ & 0.289 & 5.32 & 0.054 & 3.64 & 0.009 \\
\hline Water availability & 0.252 & 4.63 & 0.048 & 3.25 & 0.007 \\
\hline Water quality & 0.100 & 1.84 & 0.019 & 1.27 & 0.003 \\
\hline Proportion emergent vegetation & 0.055 & 1.01 & 0.010 & 0.69 & 0.002 \\
\hline Proportion floating vegetation & 0.011 & 0.20 & 0.002 & 0.14 & $<0.001$ \\
\hline Other sources of mortality & 0.007 & 0.12 & 0.001 & 0.09 & $<0.001$ \\
\hline Proportion submerged vegetation & 0.004 & 0.08 & 0.001 & 0.06 & $<0.001$ \\
\hline Winter refuge availability & 0.001 & 0.02 & $<0.001$ & 0.01 & $<0.001$ \\
\hline Proportion terrestrial vegetation & 0.001 & 0.02 & $<0.001$ & 0.01 & $<0.001$ \\
\hline Competitor effects & 0.001 & 0.01 & $<0.001$ & 0.01 & $<0.001$ \\
\hline
\end{tabular}

Because of the influence of demographic parameters on the population growth of giant gartersnakes, we also examined the sensitivity of these parameters to other variables. For adult survival, habitat quality was most influential, followed by predators, parasites, and pathogens (table 2). Water and prey availability also were influential for adult survival (table 2). First-year survival followed similar qualitative patterns, but the influence of other variables was greater than for adult survival. In particular, habitat quality and predators, parasites, and pathogens strongly influenced first-year survival, with water and prey availability also exerting an influence on this node (table 3). In contrast to survival, prey availability was the most influential node for determining giant gartersnake fecundity (table 4). Of the remaining variables, only water quality and water availability influenced fecundity (table 4). 
Table 2. Sensitivity analysis diagnostics applied to the Bayesian network model for adult survival of giant gartersnakes (Thamnophis gigas) in the Sacramento Valley, California, with uninformative (uniform) priors on all parent nodes.

[Nodes are listed in order of decreasing influence on adult survival. For all measures, greater values represent greater influence on adult survival. Adult survival is included for reference, as it represents the maximum variance reduction (VR) and mutual information (MI). <, less than]

\begin{tabular}{|c|c|c|c|c|c|}
\hline Node & $\begin{array}{l}\text { Variance } \\
\text { reduction }\end{array}$ & Percent VR & $\begin{array}{c}\text { Mutual } \\
\text { information }\end{array}$ & Percent MI & $\begin{array}{l}\text { Variance of } \\
\text { beliefs }\end{array}$ \\
\hline Giant gartersnake adult survival & 0.082 & 100.00 & 1.561 & 100.00 & 0.433 \\
\hline $\begin{array}{l}\text { Giant gartersnake population } \\
\text { growth }\end{array}$ & 0.021 & 25.20 & 0.260 & 16.60 & 0.047 \\
\hline Habitat quality & 0.013 & 15.40 & 0.165 & 10.60 & 0.027 \\
\hline $\begin{array}{l}\text { Predators, parasites, and } \\
\text { pathogens }\end{array}$ & 0.010 & 11.80 & 0.123 & 7.90 & 0.017 \\
\hline $\begin{array}{l}\text { Giant gartersnake first-year } \\
\text { survival }\end{array}$ & 0.007 & 8.19 & 0.084 & 5.36 & 0.014 \\
\hline Water availability & 0.006 & 7.31 & 0.081 & 5.20 & 0.009 \\
\hline Prey availability & 0.003 & 3.84 & 0.041 & 2.62 & 0.006 \\
\hline Proportion emergent vegetation & 0.001 & 1.65 & 0.018 & 1.13 & 0.002 \\
\hline Water quality & 0.001 & 1.42 & 0.017 & 1.12 & 0.002 \\
\hline Giant gartersnake fecundity & 0.001 & 0.84 & 0.010 & 0.62 & 0.001 \\
\hline Other sources of mortality & $<0.001$ & 0.39 & 0.005 & 0.34 & 0.001 \\
\hline Proportion submerged vegetation & $<0.001$ & 0.21 & 0.002 & 0.16 & $<0.001$ \\
\hline Proportion floating vegetation & $<0.001$ & 0.13 & 0.002 & 0.10 & $<0.001$ \\
\hline Winter refuge availability & $<0.001$ & 0.04 & $<0.001$ & 0.03 & $<0.001$ \\
\hline Proportion terrestrial vegetation & $<0.001$ & 0.04 & $<0.001$ & 0.03 & $<0.001$ \\
\hline Competitor effects & $<0.001$ & $<0.01$ & $<0.001$ & $<0.01$ & $<0.001$ \\
\hline
\end{tabular}


Table 3. Sensitivity analysis diagnostics applied to the Bayesian network model for first-year survival of giant gartersnakes (Thamnophis gigas) in the Sacramento Valley, California, with uninformative (uniform) priors on all parent nodes.

[Nodes are listed in order of decreasing influence on first-year survival. For all measures, greater values represent greater influence on first-year survival. First-year survival is included for reference, as it represents the maximum variance reduction (VR) and mutual information (MI). <, less than]

\begin{tabular}{|c|c|c|c|c|c|}
\hline Node & $\begin{array}{l}\text { Variance } \\
\text { reduction }\end{array}$ & Percent VR & $\begin{array}{c}\text { Mutual } \\
\text { information }\end{array}$ & Percent MI & $\begin{array}{c}\text { Variance of } \\
\text { beliefs }\end{array}$ \\
\hline $\begin{array}{l}\text { Giant gartersnake first-year } \\
\text { survival }\end{array}$ & 0.062 & 100.00 & 1.514 & 100.00 & 0.413 \\
\hline Habitat quality & 0.015 & 23.70 & 0.206 & 13.60 & 0.032 \\
\hline $\begin{array}{l}\text { Predators, parasites, and } \\
\text { pathogens }\end{array}$ & 0.012 & 20.10 & 0.200 & 13.20 & 0.036 \\
\hline Water availability & 0.007 & 11.40 & 0.110 & 7.25 & 0.013 \\
\hline $\begin{array}{l}\text { Giant gartersnake population } \\
\text { growth }\end{array}$ & 0.007 & 10.50 & 0.091 & 6.01 & 0.014 \\
\hline Giant gartersnake adult survival & 0.006 & 10.00 & 0.084 & 5.53 & 0.012 \\
\hline Prey availability & 0.005 & 8.13 & 0.066 & 4.36 & 0.009 \\
\hline Proportion emergent vegetation & 0.002 & 2.61 & 0.023 & 1.52 & 0.003 \\
\hline Water quality & 0.001 & 1.82 & 0.017 & 1.11 & 0.002 \\
\hline Giant gartersnake fecundity & 0.001 & 1.74 & 0.015 & 0.97 & 0.002 \\
\hline Other sources of mortality & $<0.001$ & 0.36 & 0.004 & 0.24 & $<0.001$ \\
\hline Proportion submerged vegetation & $<0.001$ & 0.35 & 0.003 & 0.20 & $<0.001$ \\
\hline Proportion floating vegetation & $<0.001$ & 0.30 & 0.003 & 0.18 & $<0.001$ \\
\hline Winter refuge availability & $<0.001$ & 0.07 & 0.001 & 0.04 & $<0.001$ \\
\hline Proportion terrestrial vegetation & $<0.001$ & 0.07 & 0.001 & 0.04 & $<0.001$ \\
\hline Competitor effects & $<0.001$ & 0.01 & $<0.001$ & $<0.01$ & $<0.001$ \\
\hline
\end{tabular}


Table 4. Sensitivity analysis diagnostics applied to the Bayesian network model for fecundity of giant gartersnakes (Thamnophis gigas) in the Sacramento Valley, California, with uninformative (uniform) priors on all parent nodes.

[Nodes are listed in order of decreasing influence on fecundity. For all measures, greater values represent greater influence on fecundity. Fecundity is included for reference, as it represents the maximum mutual information (MI). $<$, less than]

\begin{tabular}{lccc}
\hline \multicolumn{1}{c}{ Node } & Mutual information & Percent MI & Variance of beliefs \\
\hline Giant gartersnake fecundity & 1.850 & 100.00 & 0.502 \\
\hline Giant gartersnake population growth & 0.178 & 9.63 & 0.013 \\
\hline Prey availability & 0.157 & 8.48 & 0.008 \\
\hline Water quality & 0.033 & 1.77 & 0.003 \\
\hline Water availability & 0.024 & 1.28 & 0.001 \\
\hline Habitat quality & 0.016 & 0.87 & 0.001 \\
\hline Giant gartersnake first-year survival & 0.015 & 0.79 & 0.001 \\
\hline Giant gartersnake adult survival & 0.010 & 0.52 & 0.001 \\
\hline Proportion emergent vegetation & 0.004 & 0.22 & $<0.001$ \\
\hline Proportion floating vegetation & 0.003 & 0.18 & $<0.001$ \\
\hline Predators, parasites, and pathogens & 0.003 & 0.15 & $<0.001$ \\
\hline Competitor effects & $<0.001$ & 0.02 & $<0.001$ \\
\hline Other sources of mortality & $<0.001$ & $<0.01$ & $<0.001$ \\
\hline Proportion submerged vegetation & $<0.001$ & $<0.01$ & $<0.001$ \\
\hline Winter refuge availability & $<0.001$ & $<0.01$ & $<0.001$ \\
\hline Proportion terrestrial vegetation & $<0.001$ & $<0.01$ & $<0.001$ \\
\hline
\end{tabular}

We also examined the sensitivity of habitat quality because habitat quality had a strong influence on survival and presumably is the variable under the most direct control of resource managers. Water availability exerted the greatest influence on habitat quality, and was more than six times as influential as proportion emergent vegetation, the next most influential parent node for habitat quality (table 5). Proportion emergent vegetation, in turn, was more than 3.5 times more influential than water quality and proportion submerged vegetation, which were similar to each other in their influence on habitat quality (table 5). High sensitivities of the children of habitat quality were indicative of the central location and important role of habitat quality in the network (table 5). 
Table 5. Sensitivity analysis diagnostics applied to the Bayesian network model for habitat quality of giant gartersnakes (Thamnophis gigas) in the Sacramento Valley, California, with uninformative (uniform) priors on all parent nodes.

[Nodes are listed in order of decreasing influence on habitat quality. For all measures, greater values represent greater influence on habitat quality. Habitat quality is included for reference, as it represents the maximum mutual information (MI). <, less than]

\begin{tabular}{lccc}
\hline \multicolumn{1}{c}{ Node } & Mutual information & Percent MI & Variance of beliefs \\
\hline Habitat quality & 1.391 & 100.00 & 0.360 \\
\hline Water availability & 0.483 & 34.70 & 0.099 \\
\hline Giant gartersnake first-year survival & 0.206 & 14.80 & 0.032 \\
\hline Giant gartersnake adult survival & 0.165 & 11.90 & 0.027 \\
\hline Predators, parasites, and pathogens & 0.124 & 8.94 & 0.020 \\
\hline Prey availability & 0.090 & 6.46 & 0.015 \\
\hline Giant gartersnake population growth & 0.082 & 5.87 & 0.014 \\
\hline Proportion emergent vegetation & 0.078 & 5.63 & 0.016 \\
\hline Water quality & 0.022 & 1.59 & 0.002 \\
\hline Proportion submerged vegetation & 0.022 & 1.57 & 0.002 \\
\hline Giant gartersnake fecundity & 0.016 & 1.16 & 0.003 \\
\hline Winter refuge availability & 0.006 & 0.40 & $<0.001$ \\
\hline Proportion terrestrial vegetation & 0.006 & 0.40 & $<0.001$ \\
\hline Proportion floating vegetation & 0.005 & 0.38 & 0.001 \\
\hline Other sources of mortality & $<0.001$ & $<0.01$ & $<0.001$ \\
\hline Competitor effects & $<0.001$ & $<0.01$ & $<0.001$ \\
\hline
\end{tabular}

Different water-management scenarios produced divergent results. Ephemeral and winter water produced similar results, with deterministically low habitat quality and a high likelihood of decreasing giant gartersnake population growth (figs. 5 and 6). Groundwater only, which was assumed to be applied during the giant gartersnake active season (similar to summer water), improved habitat quality compared to ephemeral and winter water, resulting in average habitat quality but still likely resulting in decreasing population growth (fig. 7). Summer water only also resulted in average habitat quality being the most likely outcome (fig. 8). In this scenario, decreasing population growth was still the most likely outcome, but an increase in the likelihood of increasing population growth was evident relative to the groundwater only scenario (fig. 8). A permanent water scenario resulted in high habitat quality being the most likely outcome for this node (fig. 9). Although decreasing population growth was still the most likely outcome, stable and increasing populations were nearly as likely under this scenario (fig. 9). 


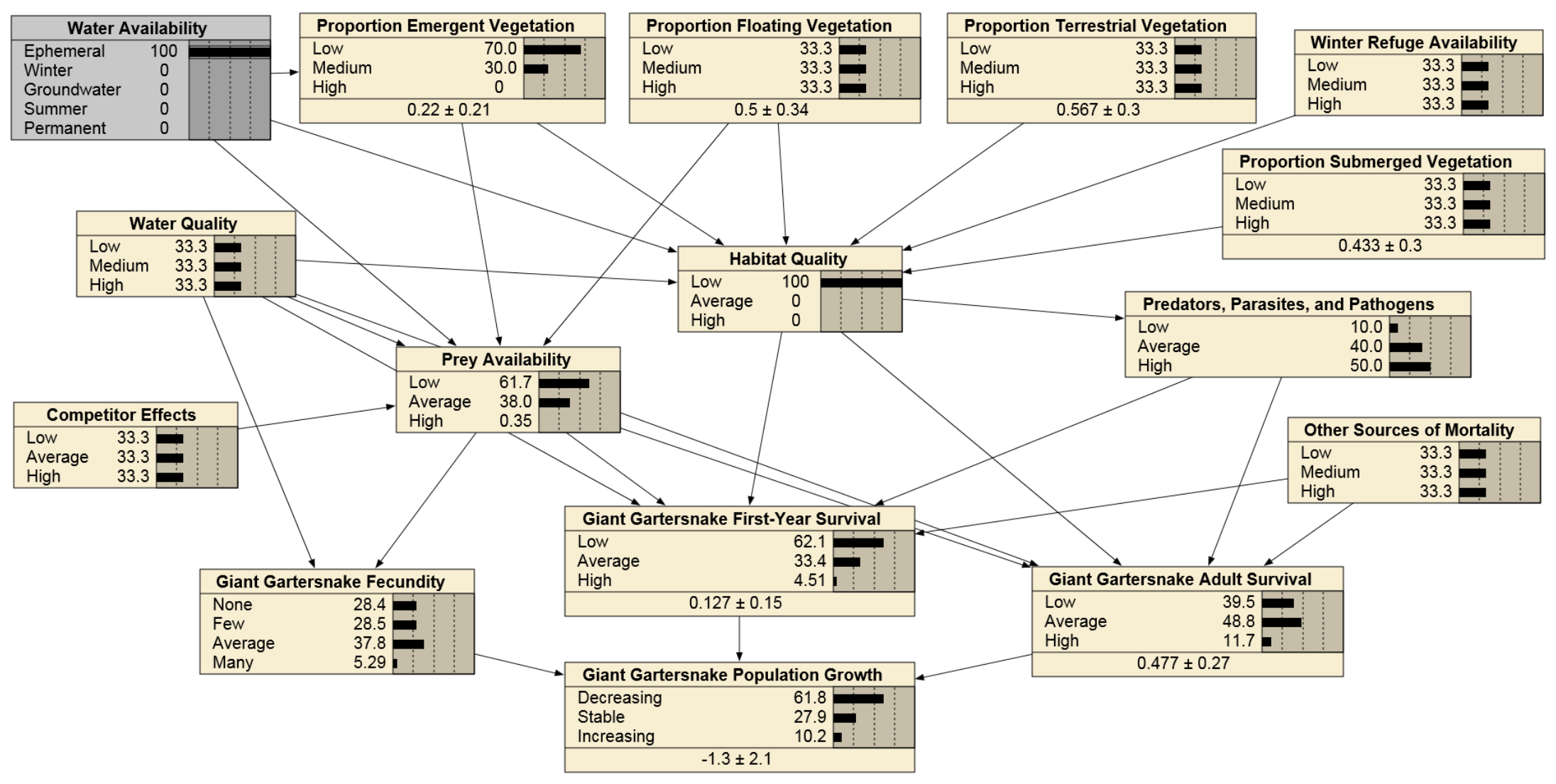

Figure 5. Most likely outcomes of the Bayesian network model for the giant gartersnake (Thamnophis gigas) in the Sacramento Valley, California, with ephemeral water-management conditions. All other parentless nodes were given uninformative (uniform) priors. Values in gray nodes were set at a fixed value to examine their influence on the remaining nodes in the network. 


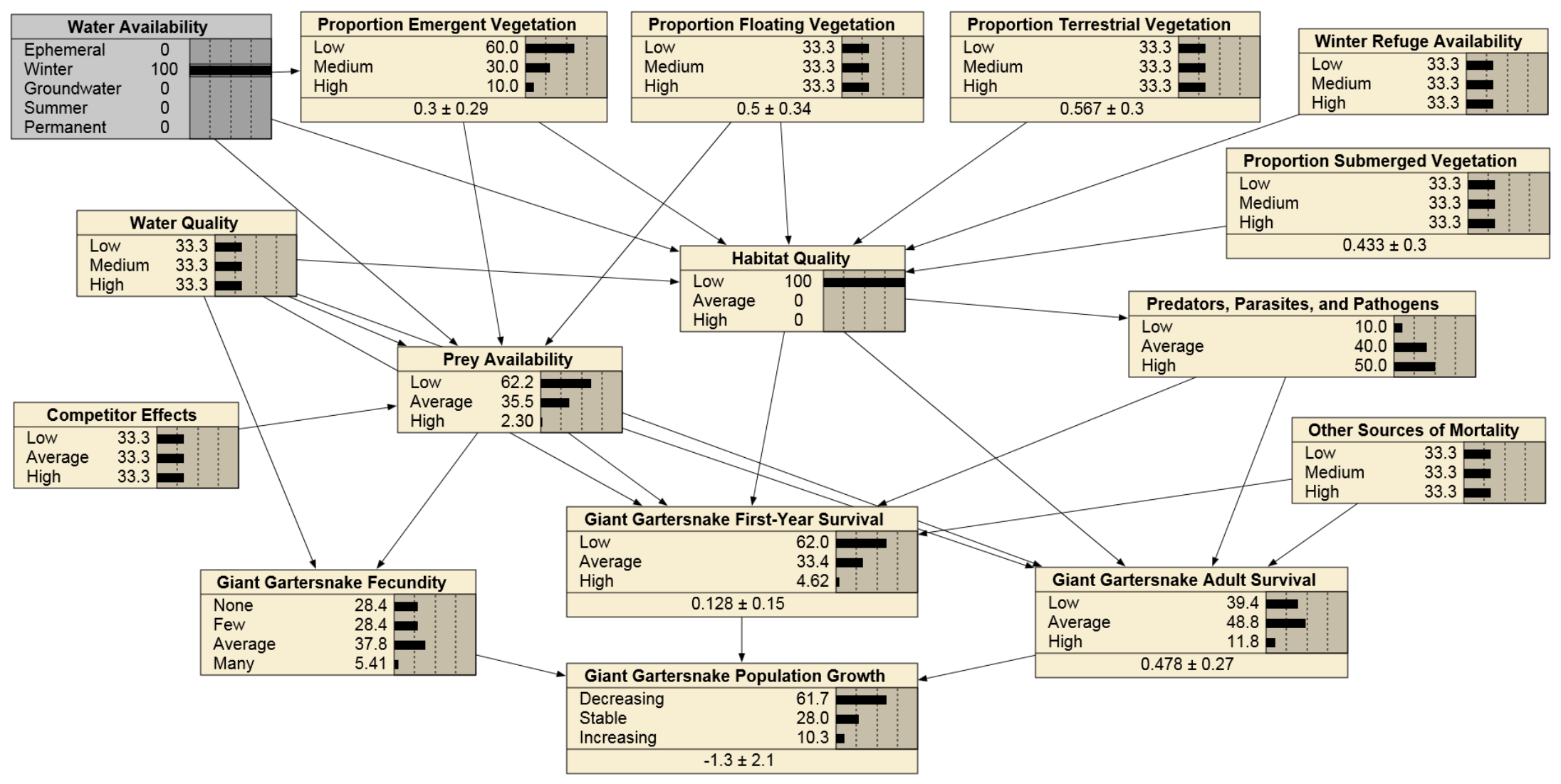

Figure 6. Most likely outcomes of the Bayesian network model for the giant gartersnake (Thamnophis gigas) in the Sacramento Valley, California, with winter water-management conditions typical for wintering waterfowl management. All other parentless nodes were given uninformative (uniform) priors. Values in gray nodes were set at a fixed value to examine their influence on the remaining nodes in the network. 


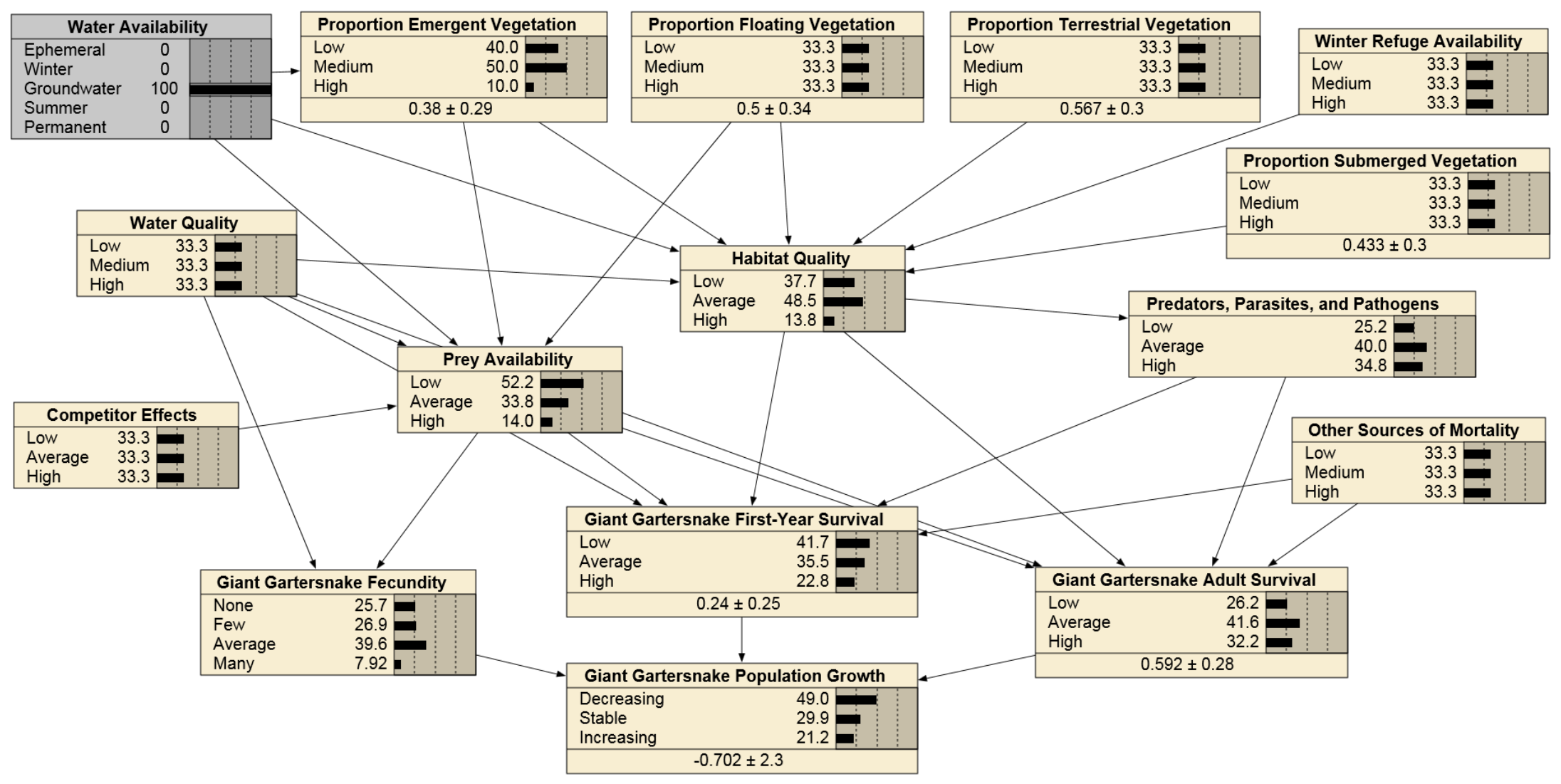

Figure 7. Most likely outcomes of the Bayesian network model for the giant gartersnake (Thamnophis gigas) in the Sacramento Valley, California, with groundwater substitution water-management conditions (assuming groundwater applied during the summer as for summer water management). All other parentless nodes were given uninformative (uniform) priors. Values in gray nodes were set at a fixed value to examine their influence on the remaining nodes in the network. 


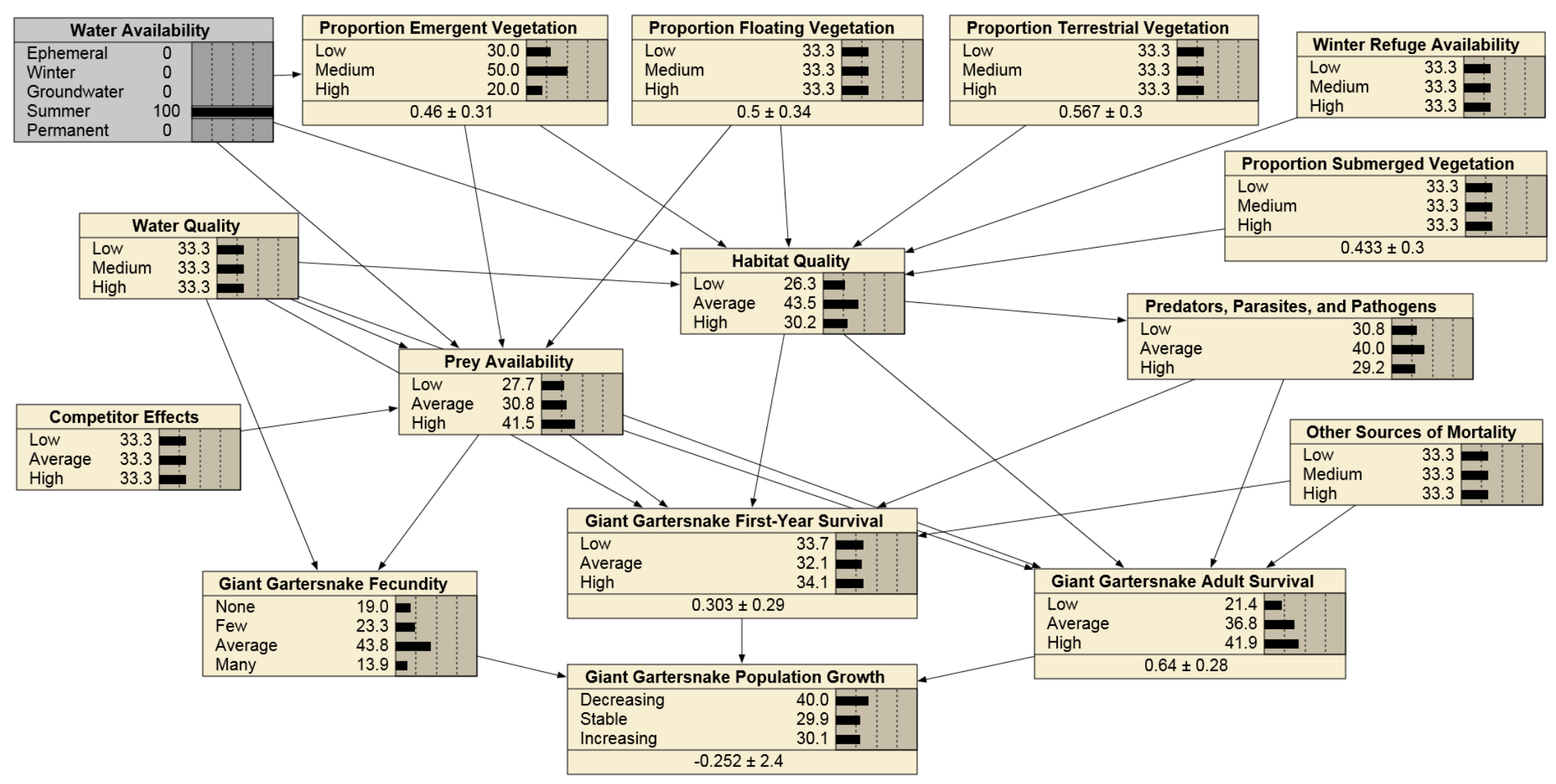

Figure 8. Most likely outcomes of the Bayesian network model for the giant gartersnake (Thamnophis gigas) in the Sacramento Valley, California, with summer water-management conditions (that is, water only available during the summer as typical of rice agriculture). All other parentless nodes were given uninformative (uniform) priors. Values in gray nodes were set at a fixed value to examine their influence on the remaining nodes in the network. 


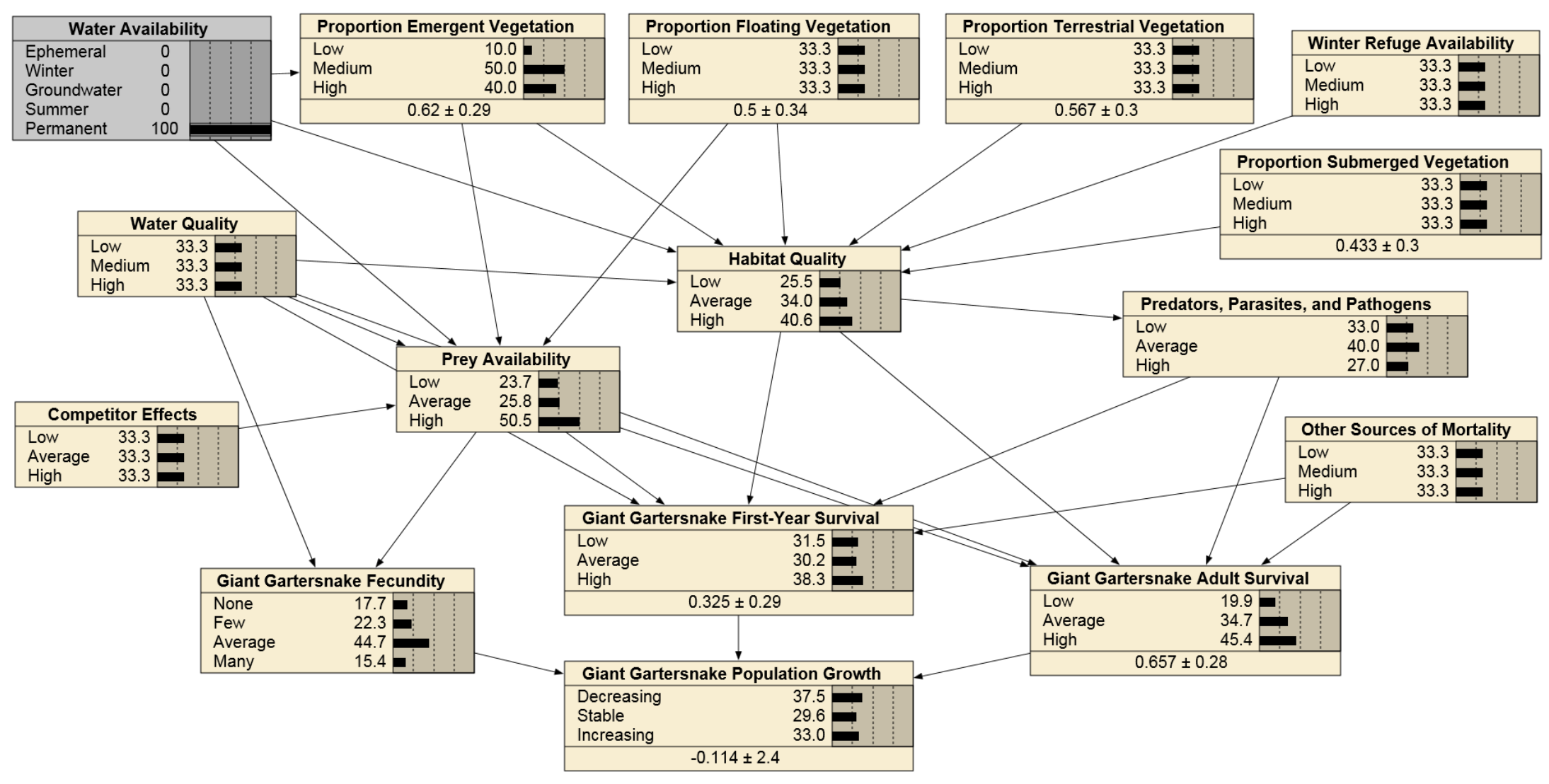

Figure 9. Most likely outcomes of the Bayesian network model for the giant gartersnake (Thamnophis gigas) in the Sacramento Valley, California, with permanent water-management conditions (that is, water in marshes all year). All other parentless nodes were given uninformative (uniform) priors. Values in gray nodes were set at a fixed value to examine their influence on the remaining nodes in the network. 
Manipulating the parentless nodes to achieve the best possible outcome for giant gartersnakes indicated the importance of high survival, high prey availability, and high habitat quality for increasing population growth (fig. 10). These conditions were fostered by permanent, high-quality water, low proportion of submerged and floating vegetation, low competitor effects and other sources of mortality, and high terrestrial vegetation and winter refuge availability (fig. 10). The worst possible outcome for giant gartersnakes, decreasing the population growth rate, was exemplified by the opposite conditions, with water only available during the winter (fig. 11).

Population growth of the giant gartersnake was most influenced by demographic parameters, especially adult survival. Directly managing for increased survival or fecundity, however, generally is not feasible. Habitat quality, which itself was strongly influenced by water availability and proportion emergent vegetation, can be managed and had a strong influence on both adult and first-year survival. Additional research into the effects of specific habitat attributes on giant gartersnake fitness is needed to better quantify the qualitative relations hypothesized in the Bayesian network model. In this regard, habitat quality; predator, parasite, and pathogen effects; and prey availability (particularly as it affects fecundity) all would be productive avenues for future research efforts.

Alternatively, research could focus on those nodes for which the least information exists. For example, the scenario analysis indicated that changing giant gartersnake population growth from increasing to decreasing resulted in little change in many nodes. Competitor effects, other sources of mortality, and nearly all parents of habitat quality were changed little under increasing and decreasing population growth scenarios. This indicates that these variables either (1) are truly unimportant for determining giant gartersnake population growth, or (2) uncertainty in the strength of these relations precludes drawing conclusions about how these variables affect population growth of the giant gartersnake. The prudent course of action would be to conduct research into the effects of these variables on giant gartersnakes to determine which of these alternatives is correct. 


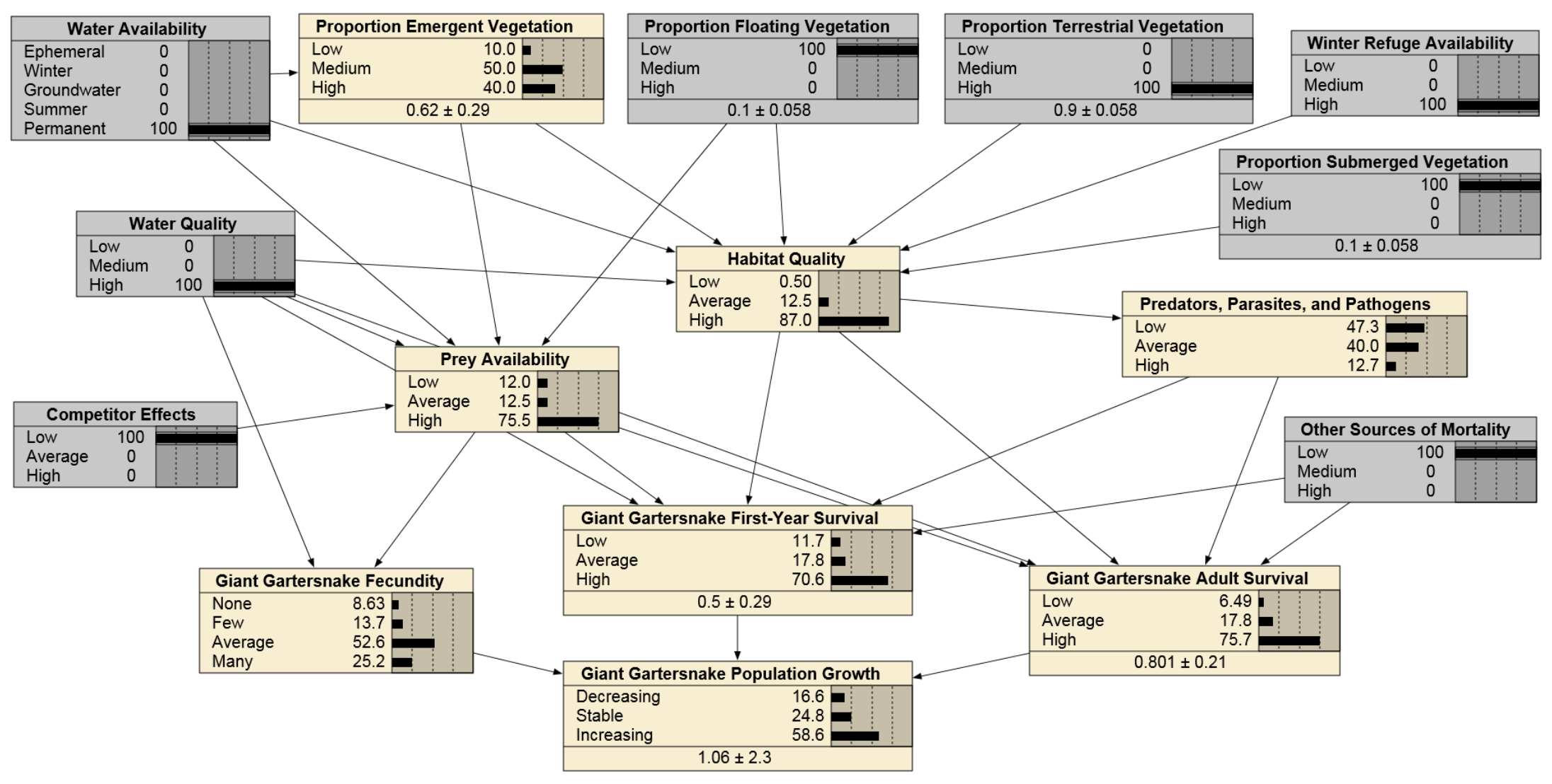

Figure 10. Best-case scenario in the Bayesian network model for increasing population growth rate of giant gartersnakes (Thamnophis gigas), in the Sacramento Valley, California. All parentless nodes were adjusted to achieve the highest possible probability of an increasing population. Values in gray nodes were set at a fixed value to examine their influence on the remaining nodes in the network. 


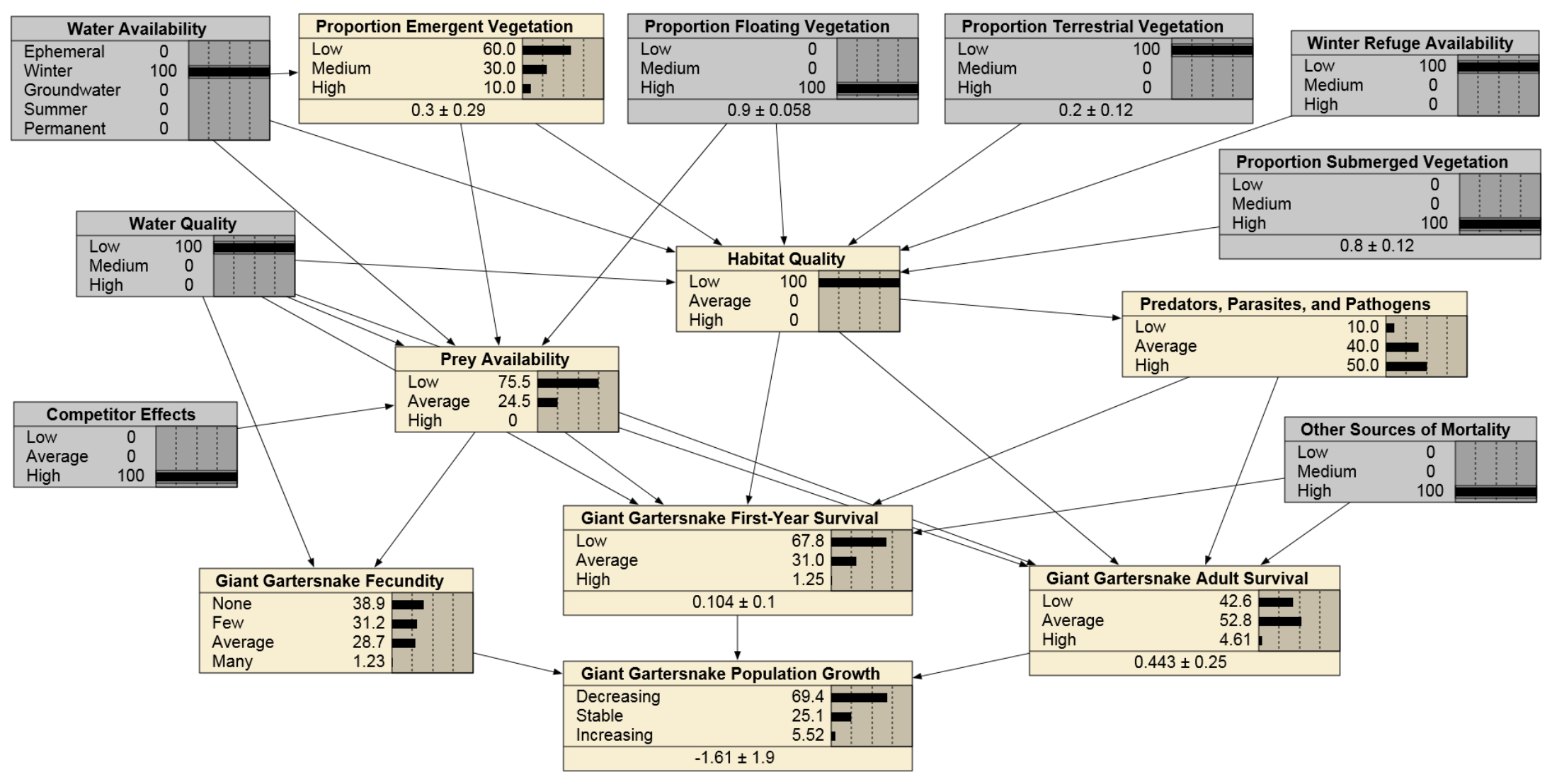

Figure 11. Worst-case scenario in the Bayesian network model for decreasing population growth rate of giant gartersnakes (Thamnophis gigas), in the Sacramento Valley, California. All parentless nodes were adjusted to achieve the highest possible probability of a decreasing population. Values in gray nodes were set at a fixed value to examine their influence on the remaining nodes in the network. 
Examining specific relations hypothesized in the model in an adaptive management (Wilhere, 2002; McCarthy and Possingham, 2007) framework perhaps is the best use of this Bayesian network model (Nyberg and others, 2006; Howes and others, 2010). The Bayesian paradigm is based on formulating a model, incorporating what is known about the parameters of the model, and updating beliefs about the model structure and values of parameters with additional information (Wade, 2000; McCarthy, 2007; Kéry, 2010; Link and Barker, 2010). Bayesian networks, therefore, are ideally suited to adaptive management, and most Bayesian network software is built to allow models to be updated with additional information. As experiments are conducted or data accumulate under varying field conditions (Shrader-Frechette and McCoy, 1993), the Bayesian network model for the giant gartersnake can be updated to inform resource managers about the best course of action to take for managing populations of giant gartersnakes. Adding decision nodes for different management actions and utility nodes to indicate the costs (in terms of water lost for other uses, habitat management costs, etc.) and benefits of specific management decisions would further enhance the model's utility for adaptive management (Marcot and others, 2001; Nyberg and others, 2006).

Despite the utility of Bayesian network models for understanding ecological relations and facilitating adaptive management, use of such models has important limitations. The ease with which expert opinion can be used to construct Bayesian network models can allow personal biases to creep into the models. This can be avoided by having independent experts review the structure and relations in the model (Marcot and others, 2006), and this was done in the present case. Another limitation of Bayesian network models is that they do not allow for feedback loops (Marcot and others, 2001; Nyberg and others, 2006; Uusitalo, 2007). For the Bayesian network model for the giant gartersnake, this means that we could not directly incorporate density dependence into the model. Although temporal cycles can be included in Bayesian networks at the cost of much complexity (Nyberg and others, 2006; Uusitalo, 2007), little evidence for density dependence exists for giant gartersnakes (Wylie and others, 2010). Such questions about model structure also should be evaluated by future research efforts.

The intent of this Bayesian network model of site-specific giant gartersnake ecology in the Sacramento Valley was to guide management efforts using the current state of knowledge, and to prioritize research into the variables most likely to affect giant gartersnake population growth rates. The model was used successfully to evaluate hypotheses about the manner in which different variables affect giant gartersnake populations, and to indicate where we need to collect additional data to test these assumptions. Thus, the model serves as a launching pad for future research, and should direct research in productive directions. The model is not a substitute for rigorous population analysis using stochastic matrix population models (Caswell, 2006) or integral projection models (Ellner and Rees, 2006; Coulson, 2012), but rather a stepping stone toward this goal.

\section{Acknowledgments}

Shannon Skalos (U.S. Geological Survey) and David Kelly (U.S. Fish and Wildlife Service) provided thoughtful reviews that improved the quality of this report. 


\section{References Cited}

California Department of Fish and Game Commission, 1971, Animals of California declared to be endangered or threatened: California Code of Federal Regulations, Title 14, Section 670.5.

Caswell, H., 2006, Matrix population models (2nd ed.):, Sunderland, Massachusetts, Sinauer Associates, Inc .

Coulson, T., 2012, Integral projections models, their construction and use in posing hypotheses in ecology: Oikos, v. 121, p. 1,337-1,350.

Ellner, S.P., and Rees, M., 2006, Integral projection models for species with complex demography: The American Naturalist, v. 167, p. 410-428.

Frayer, W.E., Peters, D.D., and Pywell, H.R., 1989, Wetlands of the California Central ValleyStatus and trends, 1939-mid-1980s: U.S. Fish and Wildlife Service, Region 1, Portland, Oregon.

Garone, P., 2007, The Fall and rise of the Wetlands of California's Great Central Valley-A historical andeEcological study of an endangered resource of the Pacific Flyway: Berkeley, University of California Press.

Halstead, B.J., Wylie, G.D., and Casazza, M.L., 2010, Habitat suitability and conservation of the giant gartersnake (Thamnophis gigas) in the Sacramento Valley of California: Copeia, v. 2010, no. 4, p. 591-599.

Halstead, B.J., Wylie, G.D., and Casazza, M.L., 2014, Ghost of habitat past-Historic habitat affects the contemporary distribution of giant garter snakes in a modified landscape: Animal Conservation, v. 17, p. 144-153.

Halstead, B.J., Wylie, G.D., Coates, P.S., Valcarcel, P., and Casazza, M.L., 2012, Bayesian shared frailty models for regional inference about wildlife survival: Animal Conservation, v. 15 , p. $117-124$.

Hansen, G.E., and Brode, J.M., 1980, Status of the giant garter snake Thamnophis couchii gigas (Fitch): Sacramento, California Department of Fish and Game, Inland Fisheries Endangered Species Program Special Publication Report Number 80-5, 14 p.

Howes, A.L., Maron, M., and Mcalpine, CA., 2010, Bayesian networks and adaptive management of wildlife habitat: Conservation Biology, v. 24, p. 974-983.

Kéry, M., 2010, Introduction to WinBUGS for ecologists-A Bayesian approach to regression, ANOVA, mixed models and related analyses: Burlington, Massachusetts, Academic Press.

Link, W.A., and Barker, R.J., 2010, Bayesian Inference: With Ecological Applications: London, Academic Press.

Marcot, B.G., Holthausen, R.S., Raphael, M.G., Rowland, M.M., and Wisdom, M.J., 2001, Using Bayesian belief networks to evaluate fish and wildlife population viability under land management alternatives from an environmental impact statement: Forest Ecology and Management, v. 153, p. 29-42. 
Marcot, B.G., Steventon, J.D., Sutherland, G.D., and McCann, R.K, 2006, Guidelines for developing and updating Bayesian belief networks applied to ecological modeling and conservation: Canadian Journal of Forest Research, v. 36, p. 3,063-3,074.

McCarthy, M.A., 2007, Bayesian methods for ecology: Cambridge, United Kingdom, Cambridge University Press.

McCarthy, M.A., and Possingham, H.P., 2007, Active adaptive management for conservation: Conservation Biology, v. 21, p. 956-963.

Nyberg, J.B., Marcot, B.G., andSulyma, R., 2006, Using Bayesian belief networks in adaptive management: Canadian Journal of Forest Research, v. 36, p. 3,104-3,116.

Rossman, D.A., Ford, N.B., and Seigel, R.A., 1996, The garter snakes-Evolution and ecology: Norman, University of Oklahoma Press.

Shrader-Frechette, K.S., and McCoy, E.D., 1993, Method in ecology-Strategies for conservation: Cambridge, United Kingdom, Cambridge University Press.

U.S. Fish and Wildlife Service, 1993, Endangered and threatened wildlife and plantsDetermination of threatened status for the giant garter snake: Federal Register , v. 58, p. 54,053-54,066.

Uusitalo, L., 2007, Advantages and challenges of Bayesian networks in environmental modelling: Ecological Modelling, v. 203, p. 312-318.

Wade, P.R., 2000, Bayesian methods in conservation biology: Conservation Biology, v. 14, p. 1,308-1,316.

Wilhere, G.F., 2002, Adaptive management in habitat conservation plans: Conservation Biology, v. 16, p. 20-29.

Wylie, G.D., Casazza, M.L., Gregory, C.J., and Halstead, B.J., 2010, Abundance and sexual size dimorphism of the Giant Gartersnake (Thamnophis gigas) in the Sacramento Valley of California: Journal of Herpetology, v. 44, p. 94-103. 


\section{Glossary}

Adaptive management: A structured, iterative process of decision making in the face of uncertainty, with the goal of reducing uncertainty over time by monitoring outcomes.

Bayesian network model: A probabilistic graphical model that represents a set of variables and their relations in a directed acyclic graph.

Child node (children of parent nodes): A node at the end of an edge (arrow) in a Bayesian network model.

Conceptual model: A diagram that shows a set of relations between variables believed to affect a target condition; a diagram that defines theoretical entities, objects, or conditions of a system and the relations between them.

Conditional probability table: A table of probabilities linking discrete, non-independent random variables to represent the marginal probability of states of a single variable with respect to the states of the others. Conditional probability tables control the relations between nodes in a Bayesian network model.

Decision node: A node in a Bayesian network model or influence diagram that represents a decision to be made. Decision nodes usually are represented as rectangles in Bayesian network models.

Demographic parameter: Any variable that can directly affect the growth rate of a population. These may be defined for specific life stages. Examples include birth and death rates, immigration, and emigration.

Deterministic: Of or relating to a process in which no randomness or uncertainty is involved in predicting the state of one variable, given knowledge of the state of another variable.

Discretize: To convert a continuous space into an equivalent discrete space for the purposes of easier calculation.

Edge: Connections between nodes in a Bayesian network model that represent dependence between variables. Edges are represented as arrows in a Bayesian network model.

Ephemeral: Short-lived.

Integral projection model: A population model that uses information on how an individual's state, including continuous states such as size or age, influences its vital rates (survival, fecundity, and so on).

Intraguild predation: The killing and eating of potential competitors. Intraguild predation represents a combination of predation and competition, because both species feed on the same prey resources and benefit from eating one another.

Matrix population model: A specific type of population model that uses matrix algebra to model the dynamics of populations.

Mutual information: A measure of the information that two random variables share. Mutual information can be thought of as measuring how much knowing one of the variables reduces uncertainty about the other. Mutual information is sometimes referred to as "entropy reduction." 
Node: A random variable in a Bayesian network model. Nodes can be observable quantities, latent variables, unknown parameters, or hypotheses. Nodes (except decision and utility nodes) usually are represented as circles or ovals in a Bayesian network model, but they are displayed in graphical form in this report to reveal the states of each node.

Parent node: A node at the beginning of an edge (arrow) in a Bayesian network model.

Parentless node: A node that is not a child of any other node in a Bayesian network model. Precinctive: A species (or smaller taxonomic unit) that is restricted to a defined geographical area.

Risk analysis: The process of defining and analyzing dangers posed to a specified outcome by potential events.

Sensitivity analysis: The study of how the uncertainty in the output of a model can be apportioned to different sources of uncertainty in its inputs.

Scenario analysis: The process of analyzing possible events by considering alternative possible outcomes, including the paths that lead to the alternative outcomes.

State: The possible values that a node can show. In most Bayesian networks, states are discrete values that a node can take.

Stochastic: Of or relating to a process involving randomness or uncertainty in observations, which are considered as a sample of one element from a probability distribution.

Utility node: A node representing the values of all potential outcomes of the parents of that node. Utility nodes also are referred to as "value nodes," and are represented in Bayesian networks as octagons, diamonds, or hexagons.

Variance reduction: The expected reduction in uncertainty in the expected real value of a variable, given the knowledge of another variable. 


\section{Appendix A. Narrative Description of Nodes, and Logic and Assumptions Underlying Conditional Probability Table Values}

1. Population Growth Rate

A. Parent Nodes

I. Adult Survival: Adult survival for most reptiles and amphibians is the life stage with the greatest effect on population growth rate. For this reason, adult survival is weighted higher than the other variables. At low adult survival (0.0-0.4 annual survival probability), population growth rate always is more likely to be decreasing than stable or increasing. Medium adult survival (0.4-0.8 annual survival probability) results in a $20 \%$ decrease in the probability of a decreasing population growth rate. At high adult survival (0.8-1.0 annual survival probability), there is a further $10 \%$ decrease in the probability of a decreasing population growth rate.

II. Juvenile Survival: Juvenile survival has less effect on population growth rate than adult survival. What constitutes low, average, and high values of juvenile survival is currently unknown. Holding all other parent nodes equal, high juvenile survival (0.3-1.0 annual survival probability) results in a 5\% increase in the probability of a stable or increasing population (and a 5\% decrease in the probability of a decreasing population) greater than that with average juvenile survival (0.1-0.3 annual survival probability). Low juvenile survival (0.0-0.1 annual survival probability) results in a further $5 \%$ decrease in the probability of stable or increasing population growth rate.

III. Fecundity: Fecundity has a large effect on population growth rate because the population cannot increase without recruitment. Regardless of survival, if fecundity is none ( 0 offspring produced), the population cannot be increasing. It can, however, be stable in the short term if survival rates are high. If all other parent nodes are held constant, many offspring results in a $20 \%$ increase in the probability that the population growth is increasing at a rate greater than that achieved with an average number of offspring. An average number of offspring, in turn, results in a $20 \%$ increase in the probability that the population growth is increasing at a rate greater than that achieved with few offspring. It remains to be determined how many offspring constitute few, average, or many.

B. Discretization
I. Decreasing $=-5.0 \leq r<-0.05$
II. Stable $=-0.05 \leq r \leq 0.05$
III. Increasing $=0.0<r \leq 5.0$ 


\section{Fecundity}

\section{A. Parent Nodes}

I. Prey Availability: Prey availability is assumed to be positively correlated to litter size with the assumption that giant gartersnakes, like other natricine snakes, are capital breeders. The probability of no young is equivalent to the probability that a female will skip reproduction; in this case, low prey availability would result in greater probability of skipping reproduction. Average prey availability is most likely to produce average litter sizes, and high prey availability is likely to increase the probability of average or many young by $10 \%$ each.

II. Water Quality: Water quality is assumed to be positively correlated to litter size (that is, relatively warm water with low concentrations of agrochemicals and heavy metals will allow females to produce larger, healthier litters) with the assumption that many chemical contaminants cause reproductive harm. Under constant prey availability, a decrease in water quality decreases the likelihood of greater categories for litter size by $5-10 \%$.

B. No discretization applied, but guidelines are as follows:
I. None $=0$
II. Few $=1-15$
III. Average $=15-20$
IV. Many $=>20$

3. Adult Survival

\section{A. Parent Nodes}

I. Prey Availability: Prey availability effects on adult survival are assumed to be less than the effects of habitat quality and similar to the effects of predators. As prey quality increases from low to average, the probability of adult survival being high increases by approximately 5-10\%. A similar increase occurs as prey availability increases from average to high. Decreases in the probability of low adult survival as prey availability increases are similar to the increases in the probability of high adult survival.

II. Predator Effects: Predator effects on adult survival are assumed to be less than the effects of habitat quality and similar to the effects of prey availability. As predator effects increase from low to average, the probability of adult survival being high decreases by approximately $5-10 \%$. A similar decrease occurs as predator effects increase from average to high. Increases in the probability of low adult survival as predator effects increase are similar to the decreases in the probability of high adult survival.

III. Habitat Quality: Direct effects of habitat quality are assumed to have the greatest effect on adult survival. As habitat quality decreases from high to average, with all other parent nodes held constant, the probability of high adult survival decreases by $45-50 \%$. Further decreases from average to low habitat quality result in an additional decrease in the probability of high adult survival of 15-25\%. Increases in the probability of low adult survival as habitat quality decreases are similar to the decreases in the probability of high adult survival. 
IV. Water Quality: Based upon the findings of Hopkins and others (1999, 2002) and Santos and others (1999), it is assumed that giant gartersnakes are less sensitive to chemical contamination than most mammals, birds, and amphibians. This category also includes water temperature and productivity, which can affect the thermal quality of the habitat and prey availability (although these actions are assumed to act indirectly through the habitat quality node). With all other nodes being equal, decreased water quality decreases the likelihood of high adult survival by $5 \%$.

V. Other Sources of Mortality: This node covers sources of mortality not dealt with elsewhere; for example, direct human killing, roadkill, mortality during routine agricultural or habitat management activity, and so on. It is assumed that these other sources of mortality are low, but in some situations they could be more important than hypothesized in the model. With all other factors being equal, increasing other sources of mortality decreases the likelihood of high adult survival by $5 \%$.

B. Discretization: Discretization is based on Halstead and others (2012).

I. Low $=0.0-0.4$ annual probability of survival

II. Average $=0.4-0.8$ annual probability of survival

III. High=0.8-1.0 annual probability of survival

4. First-Year Survival

\section{A. Parent Nodes}

I. Prey Availability: Prey availability effects on juvenile survival are assumed to be less than the effects of habitat quality and predators. As prey quality increases from low to average, the probability of juvenile survival being high increases by approximately 5-10\%. A similar increase occurs as prey availability increases from average to high. Decreases in the probability of low juvenile survival as prey availability increases are similar to the increases in the probability of high juvenile survival.

II. Predator Effects: Predator effects on juvenile survival are assumed to be stronger than the effects of predators on adults because of the smaller size and greater vulnerability of small snakes to predators. As predator effects increase from low to average, the probability of juvenile survival being low increases by approximately 20-30\%. A similar increase occurs as predator effects increase from average to high. Decreases in the probability of high adult survival as predator effects increase are smaller than the increases in the probability of low adult survival.

III. Habitat Quality: Direct effects of habitat quality are assumed to have as great an effect on juvenile survival as predator effects. As habitat quality decreases from high to average, with other parent nodes held constant, the probability of high juvenile survival decreases by 20-30\%. Further decreases from average to low habitat quality result in an additional decrease in the probability of high juvenile survival of 15-25\%. Increases in the probability of low juvenile survival as habitat quality decreases are similar to the decreases in the probability of high juvenile survival. 
IV. Water Quality: Based on the findings of Hopkins and others (1999, 2002) and Santos and others (1999), it is assumed that giant gartersnakes are less sensitive to chemical contamination than most mammals, birds, and amphibians. This category also includes water temperature and productivity, which can affect the thermal quality of the habitat and prey availability (though these actions are assumed to act indirectly through the habitat quality node). With all other nodes being equal, decreased water quality decreases the likelihood of high first-year survival by $5 \%$.

V. Other Sources of Mortality: This node covers sources of mortality not dealt with elsewhere; for example, direct human killing, roadkill, mortality during routine agricultural or habitat management activity, and so on. It is assumed that these other sources of mortality are low, but in some situations they could be more important than hypothesized in the model. All else being equal, increasing other sources of mortality decreases the likelihood of high first-year survival by $5 \%$.

B. Discretization: Discretization is a best guess.

I. Low $=0.0-0.1$ annual probability of survival

II. Average $=0.1-0.3$ annual probability of survival

III. High=0.3-1.0 annual probability of survival

5. Predators (including parasites and disease)

\section{A. Parent Nodes}

I. Habitat Quality: Habitat quality mediates predator effects through the abundance of predators (for example, perches for raptors) and the efficiency with which predators can prey on giant gartersnakes (less cover/more exposure and simple geometries such as canals that can increase predator efficiency). Low habitat quality increases the probability of severe predator effects ( $40 \%$ chance of average predator effects, $50 \%$ of high predator effects), and high habitat quality decreases the probability of predator effects (40\% probability of average predator effects, $50 \%$ probability of low predator effects). Because predator effects are not strictly linked to habitat quality, there always is some probability of low or high predator effects.

B. Discretization: None applied.

6. Prey

\section{A. Parent Nodes}

I. Competitors: Competitors are expected to have an inverse relation with prey availability, but the relation is expected to be weak. Thus, competitors, such as bullfrogs, fishes, wading birds, and other gartersnakes, are not expected to have a large influence on prey availability. Low abundance of competitors is expected to result in an approximately 10\% higher prey availability than high abundance of competitors, with all other parent nodes being equal.

II. Water Availability/Seasonality: Water availability has the greatest effect on prey availability. Permanent water provides the greatest probability of having abundant prey. Summer water prey availability is assumed to be about one-half of permanent water prey availability. Winter and ephemeral water result further decreases in prey availability. 
III. Percent Cover of Emergent Vegetation: Percent cover of emergent vegetation is expected to have a small, positive effect on prey availability because of the refuge it provides for small prey items. Increasing emergent vegetation cover from low to medium and medium to high increases the probability of average and high prey availability by $5 \%$ at each step, and decreases the probability of low prey availability by $10 \%$ at each step.

IV. Percent Cover of Floating Vegetation: Percent cover of floating vegetation is expected to have a small, negative effect on prey availability because of the potential negative alterations to aquatic habitats (reduced light penetration, etc.). Increasing floating vegetation cover from low to medium and medium to high decreases the probability of average and high prey availability by $5 \%$ at each step, and increases the probability of low prey availability by $10 \%$ at each step.

V. Water Quality: Water quality is assumed to affect prey communities based upon temperature and productivity (that is, warm, productive surface water compared to cold groundwater with few prey), as well as chemical contamination reducing or eliminating prey populations. With all other nodes being equal, a unit decrease in water quality decreases the likelihood of high prey availability by $5-10 \%$.

B. Discretization: None Applied.

7. Habitat Quality

\section{A. Parent Nodes}

I. Water Availability/Seasonality: Ephemeral water availability, or water that is only available in the winter, results in low habitat quality, regardless of the values of other parent nodes. With all other parent nodes being equal, summer water results in lower habitat quality than permanent water, but only by about 10 percent (that is, permanent water generally results in a $10 \%$ higher probability of high-quality habitat and a $10 \%$ lower probability of low quality habitat than summer water with the other parent nodes fixed).

II. Water Quality: Water quality affects habitat quality as a primary driver of the thermal quality of habitat. It also facilitates or inhibits the growth of different types of vegetation, depending upon the timing and levels of agricultural runoff (herbicides, fertilizers, and so on). With all other nodes being equal, a unit increase in water quality increases habitat quality by $10-20 \%$.

III. Percent Cover of Emergent Vegetation: Medium (20-80\%) cover of emergent vegetation results in the highest habitat quality, with high (80-100\%) cover resulting in a slight decrease in the probability of habitat being of high quality. Because of the importance of emergent vegetation as cover for giant gartersnakes, low (0-20\%) cover results in a decrease of $40-60 \%$ in the probability of habitat being of high quality relative to medium cover, and similar increases in the probability of habitat being of low quality.

IV. Percent Cover of Floating Vegetation: Floating vegetation (algae, Lemna spp., and Azolla spp.) has a negative effect on the probability of occurrence of giant gartersnakes, and radio telemetered individuals avoid floating vegetation (U.S. Geological Survey, unpub. data, 2012). Low (0-20\%) cover of floating vegetation results in the greatest probability of high-quality habitat, with medium (20-80\%) cover resulting in a decrease of $10 \%$ in the probability of habitat being of high 
quality and high (80-100 percent) cover resulting in an additional 20\% decrease in the probability of habitat being of high quality. Increases in the probability of habitat being of low quality with increasing percent cover of floating vegetation were of less magnitude.

V. Percent Cover of Terrestrial Vegetation: Because it provides cover for giant gartersnakes while they are in terrestrial habitats, high (80-100\%) cover of terrestrial vegetation results in the highest habitat quality. Medium (40-80\%) cover of terrestrial vegetation results in a decrease of $10 \%$ in the probability of habitat being of high quality and low (0- $40 \%$ ) cover results in an additional $20 \%$ decrease in the probability of habitat being of high quality. Increases in the probability of habitat being of low quality with decreasing percent cover of terrestrial vegetation were of less magnitude.

VI. Percent Cover of Submerged Vegetation: Submerged vegetation has a negative effect on the probability of occurrence of giant gartersnakes (U.S. Geological Survey, unpublished data, 2013; Eric Hansen, oral commun., 2014). Low (0$20 \%$ ) cover of submerged vegetation results in the greatest probability of highquality habitat, with medium (20-80\%) cover resulting in a decrease of $5-10 \%$ in the probability of habitat being of high quality and high (80-100\%) cover resulting in an additional 5-10\% decrease in the probability of habitat being of high quality. Increases in the probability of habitat being of low quality with increasing percent cover of submerged vegetation were of less magnitude.

VII. Availability of Refugia/Hibernacula: Because of the necessity of refugia (such as mammal and crayfish burrows, dense clumps of vegetation, riprap, or piles of debris, for brumation and protection from predators and environmental extremes), high availability of refugia results in the highest habitat quality. Medium availability of refugia results in a decrease of $10 \%$ in the probability of habitat being of high quality and low availability of refugia results in an additional $20 \%$ decrease in the probability of habitat being of high quality. Increases in the probability of habitat being of low quality with decreasing availability of refugia were of less magnitude.

B. Discretization: None Applied.

8. Emergent Vegetation

\section{A. Parent Nodes}

I. Water Availability/Seasonality: The potential for emergent vegetation to become established and persist increases with increasing hydroperiod. Standing water is viewed as a necessary, but not sufficient, condition for the growth of emergent vegetation; thus, the presence of standing water for longer periods of time increases the probability that emergent vegetation will have a greater percent cover.

\section{B. Discretization}
I. $\quad$ Low $=0-20 \%$ cover
II. Medium $=20-80 \%$ cover
III. High $=80-100 \%$ cover 


\section{Appendix B. Conditional Probability Tables}

Table B1. Conditional probability table (CPT) for the emergent vegetation node.

[Numbers in the table represent probabilities as percentages (that is, $70=70 \%$ or 0.70 ) that the response node will take the value in the column (low, medium, high), given the value of the parent node (water availability)]

\begin{tabular}{lccc}
\hline \multirow{2}{*}{ Water availability } & \multicolumn{2}{c}{ Proportion emergent } \\
\cline { 2 - 4 } & Low & Medium & High \\
\hline Ephemeral & 70 & 30 & 10 \\
\hline Winter & 60 & 30 & 10 \\
\hline Groundwater & 40 & 50 & 20 \\
\hline Summer & 30 & 50 & 40 \\
\hline Permanent & 10 & 50 & \\
\hline
\end{tabular}


Table B2. Conditional probability table (CPT) for the prey availability node.

[Numbers in the table represent probabilities as percentages (that is, $55=55 \%$ or 0.55 ) that the response node will take the value in the column (low, average, high), given the value of the parent nodes (competitor effects, water availability, proportion emergent vegetation, proportion floating vegetation, and water quality)]

\begin{tabular}{|c|c|c|c|c|c|c|c|}
\hline \multicolumn{5}{|c|}{ Inputs } & \multicolumn{3}{|c|}{ Prey availability } \\
\hline Competitors & $\begin{array}{c}\text { Water } \\
\text { availability }\end{array}$ & Emergent & Floating & Water quality & Low & Average & High \\
\hline Low & Ephemeral & Low & Low & Low & 55 & 45 & 0 \\
\hline Low & Ephemeral & Low & Low & Medium & 50 & 50 & 0 \\
\hline Low & Ephemeral & Low & Low & High & 50 & 45 & 5 \\
\hline Low & Ephemeral & Low & Medium & Low & 60 & 40 & 0 \\
\hline Low & Ephemeral & Low & Medium & Medium & 55 & 45 & 0 \\
\hline Low & Ephemeral & Low & Medium & High & 55 & 45 & 0 \\
\hline Low & Ephemeral & Low & High & Low & 70 & 30 & 0 \\
\hline Low & Ephemeral & Low & High & Medium & 65 & 35 & 0 \\
\hline Low & Ephemeral & Low & High & High & 65 & 35 & 0 \\
\hline Low & Ephemeral & Medium & Low & Low & 55 & 45 & 0 \\
\hline Low & Ephemeral & Medium & Low & Medium & 50 & 45 & 5 \\
\hline Low & Ephemeral & Medium & Low & High & 50 & 40 & 10 \\
\hline Low & Ephemeral & Medium & Medium & Low & 60 & 40 & 0 \\
\hline Low & Ephemeral & Medium & Medium & Medium & 55 & 45 & 0 \\
\hline Low & Ephemeral & Medium & Medium & High & 55 & 45 & 0 \\
\hline Low & Ephemeral & Medium & High & Low & 70 & 30 & 0 \\
\hline Low & Ephemeral & Medium & High & Medium & 65 & 35 & 0 \\
\hline Low & Ephemeral & Medium & High & High & 65 & 35 & 0 \\
\hline Low & Ephemeral & High & Low & Low & 60 & 40 & 0 \\
\hline Low & Ephemeral & High & Low & Medium & 55 & 45 & 0 \\
\hline Low & Ephemeral & High & Low & High & 55 & 45 & 0 \\
\hline Low & Ephemeral & High & Medium & Low & 65 & 35 & 0 \\
\hline Low & Ephemeral & High & Medium & Medium & 60 & 40 & 0 \\
\hline Low & Ephemeral & High & Medium & High & 60 & 40 & 0 \\
\hline Low & Ephemeral & High & High & Low & 75 & 25 & 0 \\
\hline Low & Ephemeral & High & High & Medium & 70 & 30 & 0 \\
\hline Low & Ephemeral & High & High & High & 70 & 30 & 0 \\
\hline Low & Winter & Low & Low & Low & 55 & 45 & 0 \\
\hline Low & Winter & Low & Low & Medium & 50 & 40 & 10 \\
\hline Low & Winter & Low & Low & High & 50 & 35 & 15 \\
\hline Low & Winter & Low & Medium & Low & 60 & 40 & 0 \\
\hline Low & Winter & Low & Medium & Medium & 55 & 45 & 0 \\
\hline Low & Winter & Low & Medium & High & 55 & 40 & 5 \\
\hline Low & Winter & Low & High & Low & 70 & 30 & 0 \\
\hline
\end{tabular}




\begin{tabular}{|c|c|c|c|c|c|c|c|}
\hline \multicolumn{5}{|c|}{ Inputs } & \multicolumn{3}{|c|}{ Prey availability } \\
\hline Competitors & $\begin{array}{c}\text { Water } \\
\text { availability }\end{array}$ & Emergent & Floating & Water quality & Low & Average & High \\
\hline Low & Winter & Low & High & Medium & 65 & 35 & 0 \\
\hline Low & Winter & Low & High & High & 65 & 35 & 0 \\
\hline Low & Winter & Medium & Low & Low & 55 & 40 & 5 \\
\hline Low & Winter & Medium & Low & Medium & 50 & 35 & 15 \\
\hline Low & Winter & Medium & Low & High & 50 & 30 & 20 \\
\hline Low & Winter & Medium & Medium & Low & 60 & 40 & 0 \\
\hline Low & Winter & Medium & Medium & Medium & 55 & 40 & 5 \\
\hline Low & Winter & Medium & Medium & High & 55 & 35 & 10 \\
\hline Low & Winter & Medium & High & Low & 70 & 30 & 0 \\
\hline Low & Winter & Medium & High & Medium & 65 & 35 & 0 \\
\hline Low & Winter & Medium & High & High & 65 & 35 & 0 \\
\hline Low & Winter & High & Low & Low & 60 & 40 & 0 \\
\hline Low & Winter & High & Low & Medium & 55 & 40 & 5 \\
\hline Low & Winter & High & Low & High & 55 & 35 & 10 \\
\hline Low & Winter & High & Medium & Low & 65 & 35 & 0 \\
\hline Low & Winter & High & Medium & Medium & 60 & 40 & 0 \\
\hline Low & Winter & High & Medium & High & 60 & 40 & 0 \\
\hline Low & Winter & High & High & Low & 75 & 25 & 0 \\
\hline Low & Winter & High & High & Medium & 70 & 30 & 0 \\
\hline Low & Winter & High & High & High & 70 & 30 & 0 \\
\hline Low & Groundwater & Low & Low & Low & 45 & 35 & 20 \\
\hline Low & Groundwater & Low & Low & Medium & 40 & 30 & 30 \\
\hline Low & Groundwater & Low & Low & High & 40 & 25 & 35 \\
\hline Low & Groundwater & Low & Medium & Low & 50 & 40 & 10 \\
\hline Low & Groundwater & Low & Medium & Medium & 45 & 35 & 20 \\
\hline Low & Groundwater & Low & Medium & High & 45 & 30 & 25 \\
\hline Low & Groundwater & Low & High & Low & 60 & 40 & 0 \\
\hline Low & Groundwater & Low & High & Medium & 55 & 45 & 0 \\
\hline Low & Groundwater & Low & High & High & 55 & 40 & 5 \\
\hline Low & Groundwater & Medium & Low & Low & 45 & 30 & 25 \\
\hline Low & Groundwater & Medium & Low & Medium & 40 & 25 & 35 \\
\hline Low & Groundwater & Medium & Low & High & 40 & 20 & 40 \\
\hline Low & Groundwater & Medium & Medium & Low & 50 & 35 & 15 \\
\hline Low & Groundwater & Medium & Medium & Medium & 45 & 30 & 25 \\
\hline Low & Groundwater & Medium & Medium & High & 45 & 25 & 30 \\
\hline Low & Groundwater & Medium & High & Low & 60 & 40 & 0 \\
\hline Low & Groundwater & Medium & High & Medium & 55 & 40 & 5 \\
\hline Low & Groundwater & Medium & High & High & 55 & 35 & 10 \\
\hline Low & Groundwater & High & Low & Low & 50 & 35 & 15 \\
\hline
\end{tabular}




\begin{tabular}{|c|c|c|c|c|c|c|c|}
\hline \multicolumn{5}{|c|}{ Inputs } & \multicolumn{3}{|c|}{ Prey availability } \\
\hline Competitors & $\begin{array}{c}\text { Water } \\
\text { availability }\end{array}$ & Emergent & Floating & Water quality & Low & Average & High \\
\hline Low & Groundwater & High & Low & Medium & 45 & 30 & 25 \\
\hline Low & Groundwater & High & Low & High & 45 & 25 & 30 \\
\hline Low & Groundwater & High & Medium & Low & 55 & 40 & 5 \\
\hline Low & Groundwater & High & Medium & Medium & 50 & 35 & 15 \\
\hline Low & Groundwater & High & Medium & High & 50 & 30 & 20 \\
\hline Low & Groundwater & High & High & Low & 65 & 35 & 0 \\
\hline Low & Groundwater & High & High & Medium & 60 & 40 & 0 \\
\hline Low & Groundwater & High & High & High & 60 & 40 & 0 \\
\hline Low & Summer & Low & Low & Low & 20 & 30 & 50 \\
\hline Low & Summer & Low & Low & Medium & 15 & 25 & 60 \\
\hline Low & Summer & Low & Low & High & 15 & 20 & 65 \\
\hline Low & Summer & Low & Medium & Low & 25 & 35 & 40 \\
\hline Low & Summer & Low & Medium & Medium & 20 & 30 & 50 \\
\hline Low & Summer & Low & Medium & High & 20 & 25 & 55 \\
\hline Low & Summer & Low & High & Low & 35 & 45 & 20 \\
\hline Low & Summer & Low & High & Medium & 30 & 40 & 30 \\
\hline Low & Summer & Low & High & High & 30 & 35 & 35 \\
\hline Low & Summer & Medium & Low & Low & 20 & 25 & 55 \\
\hline Low & Summer & Medium & Low & Medium & 15 & 20 & 65 \\
\hline Low & Summer & Medium & Low & High & 15 & 15 & 70 \\
\hline Low & Summer & Medium & Medium & Low & 25 & 30 & 45 \\
\hline Low & Summer & Medium & Medium & Medium & 20 & 25 & 55 \\
\hline Low & Summer & Medium & Medium & High & 20 & 20 & 60 \\
\hline Low & Summer & Medium & High & Low & 35 & 40 & 25 \\
\hline Low & Summer & Medium & High & Medium & 30 & 35 & 35 \\
\hline Low & Summer & Medium & High & High & 30 & 30 & 40 \\
\hline Low & Summer & High & Low & Low & 25 & 30 & 45 \\
\hline Low & Summer & High & Low & Medium & 20 & 25 & 55 \\
\hline Low & Summer & High & Low & High & 20 & 20 & 60 \\
\hline Low & Summer & High & Medium & Low & 30 & 35 & 35 \\
\hline Low & Summer & High & Medium & Medium & 25 & 30 & 45 \\
\hline Low & Summer & High & Medium & High & 25 & 25 & 50 \\
\hline Low & Summer & High & High & Low & 40 & 45 & 15 \\
\hline Low & Summer & High & High & Medium & 35 & 40 & 25 \\
\hline Low & Summer & High & High & High & 35 & 35 & 30 \\
\hline Low & Permanent & Low & Low & Low & 15 & 25 & 60 \\
\hline Low & Permanent & Low & Low & Medium & 10 & 20 & 70 \\
\hline Low & Permanent & Low & Low & High & 10 & 15 & 75 \\
\hline Low & Permanent & Low & Medium & Low & 20 & 30 & 50 \\
\hline
\end{tabular}




\begin{tabular}{|c|c|c|c|c|c|c|c|}
\hline \multicolumn{5}{|c|}{ Inputs } & \multicolumn{3}{|c|}{ Prey availability } \\
\hline Competitors & $\begin{array}{c}\text { Water } \\
\text { availability }\end{array}$ & Emergent & Floating & Water quality & Low & Average & High \\
\hline Low & Permanent & Low & Medium & Medium & 15 & 25 & 60 \\
\hline Low & Permanent & Low & Medium & High & 15 & 20 & 65 \\
\hline Low & Permanent & Low & High & Low & 30 & 40 & 30 \\
\hline Low & Permanent & Low & High & Medium & 25 & 35 & 40 \\
\hline Low & Permanent & Low & High & High & 25 & 30 & 45 \\
\hline Low & Permanent & Medium & Low & Low & 15 & 20 & 65 \\
\hline Low & Permanent & Medium & Low & Medium & 10 & 15 & 75 \\
\hline Low & Permanent & Medium & Low & High & 10 & 10 & 80 \\
\hline Low & Permanent & Medium & Medium & Low & 20 & 25 & 55 \\
\hline Low & Permanent & Medium & Medium & Medium & 15 & 20 & 65 \\
\hline Low & Permanent & Medium & Medium & High & 15 & 15 & 70 \\
\hline Low & Permanent & Medium & High & Low & 30 & 35 & 35 \\
\hline Low & Permanent & Medium & High & Medium & 25 & 30 & 45 \\
\hline Low & Permanent & Medium & High & High & 25 & 25 & 50 \\
\hline Low & Permanent & High & Low & Low & 20 & 25 & 55 \\
\hline Low & Permanent & High & Low & Medium & 15 & 20 & 65 \\
\hline Low & Permanent & High & Low & High & 15 & 15 & 70 \\
\hline Low & Permanent & High & Medium & Low & 25 & 30 & 45 \\
\hline Low & Permanent & High & Medium & Medium & 20 & 25 & 55 \\
\hline Low & Permanent & High & Medium & High & 20 & 20 & 60 \\
\hline Low & Permanent & High & High & Low & 35 & 40 & 25 \\
\hline Low & Permanent & High & High & Medium & 30 & 35 & 35 \\
\hline Low & Permanent & High & High & High & 30 & 30 & 40 \\
\hline Average & Ephemeral & Low & Low & Low & 60 & 40 & 0 \\
\hline Average & Ephemeral & Low & Low & Medium & 55 & 45 & 0 \\
\hline Average & Ephemeral & Low & Low & High & 55 & 45 & 0 \\
\hline Average & Ephemeral & Low & Medium & Low & 65 & 35 & 0 \\
\hline Average & Ephemeral & Low & Medium & Medium & 60 & 40 & 0 \\
\hline Average & Ephemeral & Low & Medium & High & 60 & 40 & 0 \\
\hline Average & Ephemeral & Low & High & Low & 75 & 25 & 0 \\
\hline Average & Ephemeral & Low & High & Medium & 70 & 30 & 0 \\
\hline Average & Ephemeral & Low & High & High & 70 & 30 & 0 \\
\hline Average & Ephemeral & Medium & Low & Low & 60 & 40 & 0 \\
\hline Average & Ephemeral & Medium & Low & Medium & 55 & 45 & 0 \\
\hline Average & Ephemeral & Medium & Low & High & 55 & 40 & 5 \\
\hline Average & Ephemeral & Medium & Medium & Low & 65 & 35 & 0 \\
\hline Average & Ephemeral & Medium & Medium & Medium & 60 & 40 & 0 \\
\hline Average & Ephemeral & Medium & Medium & High & 60 & 40 & 0 \\
\hline Average & Ephemeral & Medium & High & Low & 75 & 25 & 0 \\
\hline
\end{tabular}




\begin{tabular}{|c|c|c|c|c|c|c|c|}
\hline \multicolumn{5}{|c|}{ Inputs } & \multicolumn{3}{|c|}{ Prey availability } \\
\hline Competitors & $\begin{array}{c}\text { Water } \\
\text { availability }\end{array}$ & Emergent & Floating & Water quality & Low & Average & High \\
\hline Average & Ephemeral & Medium & High & Medium & 70 & 30 & 0 \\
\hline Average & Ephemeral & Medium & High & High & 70 & 30 & 0 \\
\hline Average & Ephemeral & High & Low & Low & 65 & 35 & 0 \\
\hline Average & Ephemeral & High & Low & Medium & 60 & 40 & 0 \\
\hline Average & Ephemeral & High & Low & High & 60 & 40 & 0 \\
\hline Average & Ephemeral & High & Medium & Low & 70 & 30 & 0 \\
\hline Average & Ephemeral & High & Medium & Medium & 65 & 35 & 0 \\
\hline Average & Ephemeral & High & Medium & High & 65 & 35 & 0 \\
\hline Average & Ephemeral & High & High & Low & 80 & 20 & 0 \\
\hline Average & Ephemeral & High & High & Medium & 75 & 25 & 0 \\
\hline Average & Ephemeral & High & High & High & 75 & 25 & 0 \\
\hline Average & Winter & Low & Low & Low & 60 & 40 & 0 \\
\hline Average & Winter & Low & Low & Medium & 55 & 40 & 5 \\
\hline Average & Winter & Low & Low & High & 55 & 35 & 10 \\
\hline Average & Winter & Low & Medium & Low & 65 & 35 & 0 \\
\hline Average & Winter & Low & Medium & Medium & 60 & 40 & 0 \\
\hline Average & Winter & Low & Medium & High & 60 & 40 & 0 \\
\hline Average & Winter & Low & High & Low & 75 & 25 & 0 \\
\hline Average & Winter & Low & High & Medium & 70 & 30 & 0 \\
\hline Average & Winter & Low & High & High & 70 & 30 & 0 \\
\hline Average & Winter & Medium & Low & Low & 60 & 40 & 0 \\
\hline Average & Winter & Medium & Low & Medium & 55 & 35 & 10 \\
\hline Average & Winter & Medium & Low & High & 55 & 30 & 15 \\
\hline Average & Winter & Medium & Medium & Low & 65 & 35 & 0 \\
\hline Average & Winter & Medium & Medium & Medium & 60 & 40 & 0 \\
\hline Average & Winter & Medium & Medium & High & 60 & 35 & 5 \\
\hline Average & Winter & Medium & High & Low & 75 & 25 & 0 \\
\hline Average & Winter & Medium & High & Medium & 70 & 30 & 0 \\
\hline Average & Winter & Medium & High & High & 70 & 30 & 0 \\
\hline Average & Winter & High & Low & Low & 65 & 35 & 0 \\
\hline Average & Winter & High & Low & Medium & 60 & 40 & 0 \\
\hline Average & Winter & High & Low & High & 60 & 35 & 5 \\
\hline Average & Winter & High & Medium & Low & 70 & 30 & 0 \\
\hline Average & Winter & High & Medium & Medium & 65 & 35 & 0 \\
\hline Average & Winter & High & Medium & High & 65 & 35 & 0 \\
\hline Average & Winter & High & High & Low & 80 & 20 & 0 \\
\hline Average & Winter & High & High & Medium & 75 & 25 & 0 \\
\hline Average & Winter & High & High & High & 75 & 25 & 0 \\
\hline Average & Groundwater & Low & Low & Low & 50 & 35 & 15 \\
\hline
\end{tabular}




\begin{tabular}{|c|c|c|c|c|c|c|c|}
\hline \multicolumn{5}{|c|}{ Inputs } & \multicolumn{3}{|c|}{ Prey availability } \\
\hline Competitors & $\begin{array}{c}\text { Water } \\
\text { availability }\end{array}$ & Emergent & Floating & Water quality & Low & Average & High \\
\hline Average & Groundwater & Low & Low & Medium & 45 & 30 & 25 \\
\hline Average & Groundwater & Low & Low & High & 45 & 25 & 30 \\
\hline Average & Groundwater & Low & Medium & Low & 55 & 40 & 5 \\
\hline Average & Groundwater & Low & Medium & Medium & 50 & 35 & 15 \\
\hline Average & Groundwater & Low & Medium & High & 50 & 30 & 20 \\
\hline Average & Groundwater & Low & High & Low & 65 & 35 & 0 \\
\hline Average & Groundwater & Low & High & Medium & 60 & 40 & 0 \\
\hline Average & Groundwater & Low & High & High & 60 & 40 & 0 \\
\hline Average & Groundwater & Medium & Low & Low & 50 & 30 & 20 \\
\hline Average & Groundwater & Medium & Low & Medium & 45 & 25 & 30 \\
\hline Average & Groundwater & Medium & Low & High & 45 & 20 & 35 \\
\hline Average & Groundwater & Medium & Medium & Low & 55 & 35 & 10 \\
\hline Average & Groundwater & Medium & Medium & Medium & 50 & 30 & 20 \\
\hline Average & Groundwater & Medium & Medium & High & 50 & 25 & 25 \\
\hline Average & Groundwater & Medium & High & Low & 65 & 35 & 0 \\
\hline Average & Groundwater & Medium & High & Medium & 60 & 40 & 0 \\
\hline Average & Groundwater & Medium & High & High & 60 & 35 & 5 \\
\hline Average & Groundwater & High & Low & Low & 55 & 35 & 10 \\
\hline Average & Groundwater & High & Low & Medium & 50 & 30 & 20 \\
\hline Average & Groundwater & High & Low & High & 50 & 25 & 25 \\
\hline Average & Groundwater & High & Medium & Low & 60 & 40 & 0 \\
\hline Average & Groundwater & High & Medium & Medium & 55 & 35 & 10 \\
\hline Average & Groundwater & High & Medium & High & 55 & 30 & 15 \\
\hline Average & Groundwater & High & High & Low & 70 & 30 & 0 \\
\hline Average & Groundwater & High & High & Medium & 65 & 35 & 0 \\
\hline Average & Groundwater & High & High & High & 65 & 35 & 0 \\
\hline Average & Summer & Low & Low & Low & 25 & 30 & 45 \\
\hline Average & Summer & Low & Low & Medium & 20 & 25 & 55 \\
\hline Average & Summer & Low & Low & High & 20 & 20 & 60 \\
\hline Average & Summer & Low & Medium & Low & 30 & 35 & 35 \\
\hline Average & Summer & Low & Medium & Medium & 25 & 30 & 45 \\
\hline Average & Summer & Low & Medium & High & 25 & 25 & 50 \\
\hline Average & Summer & Low & High & Low & 40 & 45 & 15 \\
\hline Average & Summer & Low & High & Medium & 35 & 40 & 25 \\
\hline Average & Summer & Low & High & High & 35 & 35 & 30 \\
\hline Average & Summer & Medium & Low & Low & 25 & 25 & 50 \\
\hline Average & Summer & Medium & Low & Medium & 20 & 20 & 60 \\
\hline Average & Summer & Medium & Low & High & 20 & 15 & 65 \\
\hline Average & Summer & Medium & Medium & Low & 30 & 30 & 40 \\
\hline
\end{tabular}




\begin{tabular}{|c|c|c|c|c|c|c|c|}
\hline \multicolumn{5}{|c|}{ Inputs } & \multicolumn{3}{|c|}{ Prey availability } \\
\hline Competitors & $\begin{array}{c}\text { Water } \\
\text { availability }\end{array}$ & Emergent & Floating & Water quality & Low & Average & High \\
\hline Average & Summer & Medium & Medium & Medium & 25 & 25 & 50 \\
\hline Average & Summer & Medium & Medium & High & 25 & 20 & 55 \\
\hline Average & Summer & Medium & High & Low & 40 & 40 & 20 \\
\hline Average & Summer & Medium & High & Medium & 35 & 35 & 30 \\
\hline Average & Summer & Medium & High & High & 35 & 30 & 35 \\
\hline Average & Summer & High & Low & Low & 30 & 30 & 40 \\
\hline Average & Summer & High & Low & Medium & 25 & 25 & 50 \\
\hline Average & Summer & High & Low & High & 25 & 20 & 55 \\
\hline Average & Summer & High & Medium & Low & 35 & 35 & 30 \\
\hline Average & Summer & High & Medium & Medium & 30 & 30 & 40 \\
\hline Average & Summer & High & Medium & High & 30 & 25 & 45 \\
\hline Average & Summer & High & High & Low & 45 & 45 & 10 \\
\hline Average & Summer & High & High & Medium & 40 & 40 & 20 \\
\hline Average & Summer & High & High & High & 40 & 35 & 25 \\
\hline Average & Permanent & Low & Low & Low & 20 & 25 & 55 \\
\hline Average & Permanent & Low & Low & Medium & 15 & 20 & 65 \\
\hline Average & Permanent & Low & Low & High & 15 & 15 & 70 \\
\hline Average & Permanent & Low & Medium & Low & 25 & 30 & 45 \\
\hline Average & Permanent & Low & Medium & Medium & 20 & 25 & 55 \\
\hline Average & Permanent & Low & Medium & High & 20 & 20 & 60 \\
\hline Average & Permanent & Low & High & Low & 35 & 40 & 25 \\
\hline Average & Permanent & Low & High & Medium & 30 & 35 & 35 \\
\hline Average & Permanent & Low & High & High & 30 & 30 & 40 \\
\hline Average & Permanent & Medium & Low & Low & 20 & 20 & 60 \\
\hline Average & Permanent & Medium & Low & Medium & 15 & 15 & 70 \\
\hline Average & Permanent & Medium & Low & High & 15 & 10 & 75 \\
\hline Average & Permanent & Medium & Medium & Low & 25 & 25 & 50 \\
\hline Average & Permanent & Medium & Medium & Medium & 20 & 20 & 60 \\
\hline Average & Permanent & Medium & Medium & High & 20 & 15 & 65 \\
\hline Average & Permanent & Medium & High & Low & 35 & 35 & 30 \\
\hline Average & Permanent & Medium & High & Medium & 30 & 30 & 40 \\
\hline Average & Permanent & Medium & High & High & 30 & 25 & 45 \\
\hline Average & Permanent & High & Low & Low & 25 & 25 & 50 \\
\hline Average & Permanent & High & Low & Medium & 20 & 20 & 60 \\
\hline Average & Permanent & High & Low & High & 20 & 15 & 65 \\
\hline Average & Permanent & High & Medium & Low & 30 & 30 & 40 \\
\hline Average & Permanent & High & Medium & Medium & 25 & 25 & 50 \\
\hline Average & Permanent & High & Medium & High & 25 & 20 & 55 \\
\hline Average & Permanent & High & High & Low & 40 & 40 & 20 \\
\hline
\end{tabular}




\begin{tabular}{|c|c|c|c|c|c|c|c|}
\hline \multicolumn{5}{|c|}{ Inputs } & \multicolumn{3}{|c|}{ Prey availability } \\
\hline Competitors & $\begin{array}{c}\text { Water } \\
\text { availability }\end{array}$ & Emergent & Floating & Water quality & Low & Average & High \\
\hline Average & Permanent & High & High & Medium & 35 & 35 & 30 \\
\hline Average & Permanent & High & High & High & 35 & 30 & 35 \\
\hline High & Ephemeral & Low & Low & Low & 60 & 40 & 0 \\
\hline High & Ephemeral & Low & Low & Medium & 55 & 45 & 0 \\
\hline High & Ephemeral & Low & Low & High & 55 & 45 & 0 \\
\hline High & Ephemeral & Low & Medium & Low & 65 & 35 & 0 \\
\hline High & Ephemeral & Low & Medium & Medium & 60 & 40 & 0 \\
\hline High & Ephemeral & Low & Medium & High & 60 & 40 & 0 \\
\hline High & Ephemeral & Low & High & Low & 75 & 25 & 0 \\
\hline High & Ephemeral & Low & High & Medium & 70 & 30 & 0 \\
\hline High & Ephemeral & Low & High & High & 70 & 30 & 0 \\
\hline High & Ephemeral & Medium & Low & Low & 60 & 40 & 0 \\
\hline High & Ephemeral & Medium & Low & Medium & 55 & 45 & 0 \\
\hline High & Ephemeral & Medium & Low & High & 55 & 45 & 0 \\
\hline High & Ephemeral & Medium & Medium & Low & 65 & 35 & 0 \\
\hline High & Ephemeral & Medium & Medium & Medium & 60 & 40 & 0 \\
\hline High & Ephemeral & Medium & Medium & High & 60 & 40 & 0 \\
\hline High & Ephemeral & Medium & High & Low & 75 & 25 & 0 \\
\hline High & Ephemeral & Medium & High & Medium & 70 & 30 & 0 \\
\hline High & Ephemeral & Medium & High & High & 70 & 30 & 0 \\
\hline High & Ephemeral & High & Low & Low & 65 & 35 & 0 \\
\hline High & Ephemeral & High & Low & Medium & 60 & 40 & 0 \\
\hline High & Ephemeral & High & Low & High & 60 & 40 & 0 \\
\hline High & Ephemeral & High & Medium & Low & 70 & 30 & 0 \\
\hline High & Ephemeral & High & Medium & Medium & 65 & 35 & 0 \\
\hline High & Ephemeral & High & Medium & High & 65 & 35 & 0 \\
\hline High & Ephemeral & High & High & Low & 80 & 20 & 0 \\
\hline High & Ephemeral & High & High & Medium & 75 & 25 & 0 \\
\hline High & Ephemeral & High & High & High & 75 & 25 & 0 \\
\hline High & Winter & Low & Low & Low & 60 & 40 & 0 \\
\hline High & Winter & Low & Low & Medium & 55 & 45 & 0 \\
\hline High & Winter & Low & Low & High & 55 & 40 & 5 \\
\hline High & Winter & Low & Medium & Low & 65 & 35 & 0 \\
\hline High & Winter & Low & Medium & Medium & 60 & 40 & 0 \\
\hline High & Winter & Low & Medium & High & 60 & 40 & 0 \\
\hline High & Winter & Low & High & Low & 75 & 25 & 0 \\
\hline High & Winter & Low & High & Medium & 70 & 30 & 0 \\
\hline High & Winter & Low & High & High & 70 & 30 & 0 \\
\hline High & Winter & Medium & Low & Low & 60 & 40 & 0 \\
\hline
\end{tabular}




\begin{tabular}{|c|c|c|c|c|c|c|c|}
\hline \multicolumn{5}{|c|}{ Inputs } & \multicolumn{3}{|c|}{ Prey availability } \\
\hline Competitors & $\begin{array}{c}\text { Water } \\
\text { availability }\end{array}$ & Emergent & Floating & Water quality & Low & Average & High \\
\hline High & Winter & Medium & Low & Medium & 55 & 40 & 5 \\
\hline High & Winter & Medium & Low & High & 55 & 35 & 10 \\
\hline High & Winter & Medium & Medium & Low & 65 & 35 & 0 \\
\hline High & Winter & Medium & Medium & Medium & 60 & 40 & 0 \\
\hline High & Winter & Medium & Medium & High & 60 & 40 & 0 \\
\hline High & Winter & Medium & High & Low & 75 & 25 & 0 \\
\hline High & Winter & Medium & High & Medium & 70 & 30 & 0 \\
\hline High & Winter & Medium & High & High & 70 & 30 & 0 \\
\hline High & Winter & High & Low & Low & 65 & 35 & 0 \\
\hline High & Winter & High & Low & Medium & 60 & 40 & 0 \\
\hline High & Winter & High & Low & High & 60 & 40 & 0 \\
\hline High & Winter & High & Medium & Low & 70 & 30 & 0 \\
\hline High & Winter & High & Medium & Medium & 65 & 35 & 0 \\
\hline High & Winter & High & Medium & High & 65 & 35 & 0 \\
\hline High & Winter & High & High & Low & 80 & 20 & 0 \\
\hline High & Winter & High & High & Medium & 75 & 25 & 0 \\
\hline High & Winter & High & High & High & 75 & 25 & 0 \\
\hline High & Groundwater & Low & Low & Low & 50 & 40 & 10 \\
\hline High & Groundwater & Low & Low & Medium & 45 & 35 & 20 \\
\hline High & Groundwater & Low & Low & High & 45 & 30 & 25 \\
\hline High & Groundwater & Low & Medium & Low & 55 & 45 & 0 \\
\hline High & Groundwater & Low & Medium & Medium & 50 & 40 & 10 \\
\hline High & Groundwater & Low & Medium & High & 50 & 35 & 15 \\
\hline High & Groundwater & Low & High & Low & 65 & 35 & 0 \\
\hline High & Groundwater & Low & High & Medium & 60 & 40 & 0 \\
\hline High & Groundwater & Low & High & High & 60 & 40 & 0 \\
\hline High & Groundwater & Medium & Low & Low & 50 & 35 & 15 \\
\hline High & Groundwater & Medium & Low & Medium & 45 & 30 & 25 \\
\hline High & Groundwater & Medium & Low & High & 45 & 25 & 30 \\
\hline High & Groundwater & Medium & Medium & Low & 55 & 40 & 5 \\
\hline High & Groundwater & Medium & Medium & Medium & 50 & 35 & 15 \\
\hline High & Groundwater & Medium & Medium & High & 50 & 30 & 20 \\
\hline High & Groundwater & Medium & High & Low & 65 & 35 & 0 \\
\hline High & Groundwater & Medium & High & Medium & 60 & 40 & 0 \\
\hline High & Groundwater & Medium & High & High & 60 & 40 & 0 \\
\hline High & Groundwater & High & Low & Low & 55 & 40 & 5 \\
\hline High & Groundwater & High & Low & Medium & 50 & 35 & 15 \\
\hline High & Groundwater & High & Low & High & 50 & 30 & 20 \\
\hline High & Groundwater & High & Medium & Low & 60 & 40 & 0 \\
\hline
\end{tabular}




\begin{tabular}{|c|c|c|c|c|c|c|c|}
\hline \multicolumn{5}{|c|}{ Inputs } & \multicolumn{3}{|c|}{ Prey availability } \\
\hline Competitors & $\begin{array}{c}\text { Water } \\
\text { availability }\end{array}$ & Emergent & Floating & Water quality & Low & Average & High \\
\hline High & Groundwater & High & Medium & Medium & 55 & 40 & 5 \\
\hline High & Groundwater & High & Medium & High & 55 & 35 & 10 \\
\hline High & Groundwater & High & High & Low & 70 & 30 & 0 \\
\hline High & Groundwater & High & High & Medium & 65 & 35 & 0 \\
\hline High & Groundwater & High & High & High & 65 & 35 & 0 \\
\hline High & Summer & Low & Low & Low & 25 & 35 & 40 \\
\hline High & Summer & Low & Low & Medium & 20 & 30 & 50 \\
\hline High & Summer & Low & Low & High & 20 & 25 & 55 \\
\hline High & Summer & Low & Medium & Low & 30 & 40 & 30 \\
\hline High & Summer & Low & Medium & Medium & 25 & 35 & 40 \\
\hline High & Summer & Low & Medium & High & 25 & 30 & 45 \\
\hline High & Summer & Low & High & Low & 40 & 50 & 10 \\
\hline High & Summer & Low & High & Medium & 35 & 45 & 20 \\
\hline High & Summer & Low & High & High & 35 & 40 & 25 \\
\hline High & Summer & Medium & Low & Low & 25 & 30 & 45 \\
\hline High & Summer & Medium & Low & Medium & 20 & 25 & 55 \\
\hline High & Summer & Medium & Low & High & 20 & 20 & 60 \\
\hline High & Summer & Medium & Medium & Low & 30 & 35 & 35 \\
\hline High & Summer & Medium & Medium & Medium & 25 & 30 & 45 \\
\hline High & Summer & Medium & Medium & High & 25 & 25 & 50 \\
\hline High & Summer & Medium & High & Low & 40 & 45 & 15 \\
\hline High & Summer & Medium & High & Medium & 35 & 40 & 25 \\
\hline High & Summer & Medium & High & High & 35 & 35 & 30 \\
\hline High & Summer & High & Low & Low & 30 & 35 & 35 \\
\hline High & Summer & High & Low & Medium & 25 & 30 & 45 \\
\hline High & Summer & High & Low & High & 25 & 25 & 50 \\
\hline High & Summer & High & Medium & Low & 35 & 40 & 25 \\
\hline High & Summer & High & Medium & Medium & 30 & 35 & 35 \\
\hline High & Summer & High & Medium & High & 30 & 30 & 40 \\
\hline High & Summer & High & High & Low & 45 & 50 & 5 \\
\hline High & Summer & High & High & Medium & 40 & 45 & 15 \\
\hline High & Summer & High & High & High & 40 & 40 & 20 \\
\hline High & Permanent & Low & Low & Low & 20 & 30 & 50 \\
\hline High & Permanent & Low & Low & Medium & 15 & 25 & 60 \\
\hline High & Permanent & Low & Low & High & 15 & 20 & 65 \\
\hline High & Permanent & Low & Medium & Low & 25 & 35 & 40 \\
\hline High & Permanent & Low & Medium & Medium & 20 & 30 & 50 \\
\hline High & Permanent & Low & Medium & High & 20 & 25 & 55 \\
\hline High & Permanent & Low & High & Low & 35 & 45 & 20 \\
\hline
\end{tabular}




\begin{tabular}{|c|c|c|c|c|c|c|c|}
\hline \multicolumn{5}{|c|}{ Inputs } & \multicolumn{3}{|c|}{ Prey availability } \\
\hline Competitors & $\begin{array}{c}\text { Water } \\
\text { availability }\end{array}$ & Emergent & Floating & Water quality & Low & Average & High \\
\hline High & Permanent & Low & High & Medium & 30 & 40 & 30 \\
\hline High & Permanent & Low & High & High & 30 & 35 & 35 \\
\hline High & Permanent & Medium & Low & Low & 20 & 25 & 55 \\
\hline High & Permanent & Medium & Low & Medium & 15 & 20 & 65 \\
\hline High & Permanent & Medium & Low & High & 15 & 15 & 70 \\
\hline High & Permanent & Medium & Medium & Low & 25 & 30 & 45 \\
\hline High & Permanent & Medium & Medium & Medium & 20 & 25 & 55 \\
\hline High & Permanent & Medium & Medium & High & 20 & 20 & 60 \\
\hline High & Permanent & Medium & High & Low & 35 & 40 & 25 \\
\hline High & Permanent & Medium & High & Medium & 30 & 35 & 35 \\
\hline High & Permanent & Medium & High & High & 30 & 30 & 40 \\
\hline High & Permanent & High & Low & Low & 25 & 30 & 45 \\
\hline High & Permanent & High & Low & Medium & 20 & 25 & 55 \\
\hline High & Permanent & High & Low & High & 20 & 20 & 60 \\
\hline High & Permanent & High & Medium & Low & 30 & 35 & 35 \\
\hline High & Permanent & High & Medium & Medium & 25 & 30 & 45 \\
\hline High & Permanent & High & Medium & High & 25 & 25 & 50 \\
\hline High & Permanent & High & High & Low & 40 & 45 & 15 \\
\hline High & Permanent & High & High & Medium & 35 & 40 & 25 \\
\hline High & Permanent & High & High & High & 35 & 35 & 30 \\
\hline
\end{tabular}


Table B3. Conditional probability table (CPT) for the habitat quality node.

[Numbers in the table represent probabilities as percentages (that is, $100=100 \%$ or 1.00 ) that the response node will take the value in the column (low, average, high), given the value of the parent nodes (water availability, proportion emergent vegetation, proportion floating vegetation, proportion terrestrial vegetation, winter refuge availability, proportion submerged vegetation, and water quality)]

\begin{tabular}{|c|c|c|c|c|c|c|c|c|c|}
\hline \multicolumn{7}{|c|}{ Inputs } & \multicolumn{3}{|c|}{ Habitat quality } \\
\hline $\begin{array}{c}\text { Water } \\
\text { availability }\end{array}$ & Emergent & Floating & Terrestrial & $\begin{array}{l}\text { Winter } \\
\text { refuge }\end{array}$ & Submerged & $\begin{array}{l}\text { Water } \\
\text { quality }\end{array}$ & Low & Average & High \\
\hline Ephemeral & Low & Low & Low & Low & Low & Low & 100 & 0 & 0 \\
\hline Ephemeral & Low & Low & Low & Low & Low & Medium & 100 & 0 & 0 \\
\hline Ephemeral & Low & Low & Low & Low & Low & High & 100 & 0 & 0 \\
\hline Ephemeral & Low & Low & Low & Low & Medium & Low & 100 & 0 & 0 \\
\hline Ephemeral & Low & Low & Low & Low & Medium & Medium & 100 & 0 & 0 \\
\hline Ephemeral & Low & Low & Low & Low & Medium & High & 100 & 0 & 0 \\
\hline Ephemeral & Low & Low & Low & Low & High & Low & 100 & 0 & 0 \\
\hline Ephemeral & Low & Low & Low & Low & High & Medium & 100 & 0 & 0 \\
\hline Ephemeral & Low & Low & Low & Low & High & High & 100 & 0 & 0 \\
\hline Ephemeral & Low & Low & Low & Medium & Low & Low & 100 & 0 & 0 \\
\hline Ephemeral & Low & Low & Low & Medium & Low & Medium & 100 & 0 & 0 \\
\hline Ephemeral & Low & Low & Low & Medium & Low & High & 100 & 0 & 0 \\
\hline Ephemeral & Low & Low & Low & Medium & Medium & Low & 100 & 0 & 0 \\
\hline Ephemeral & Low & Low & Low & Medium & Medium & Medium & 100 & 0 & 0 \\
\hline Ephemeral & Low & Low & Low & Medium & Medium & High & 100 & 0 & 0 \\
\hline Ephemeral & Low & Low & Low & Medium & High & Low & 100 & 0 & 0 \\
\hline Ephemeral & Low & Low & Low & Medium & High & Medium & 100 & 0 & 0 \\
\hline Ephemeral & Low & Low & Low & Medium & High & High & 100 & 0 & 0 \\
\hline Ephemeral & Low & Low & Low & High & Low & Low & 100 & 0 & 0 \\
\hline Ephemeral & Low & Low & Low & High & Low & Medium & 100 & 0 & 0 \\
\hline Ephemeral & Low & Low & Low & High & Low & High & 100 & 0 & 0 \\
\hline Ephemeral & Low & Low & Low & High & Medium & Low & 100 & 0 & 0 \\
\hline Ephemeral & Low & Low & Low & High & Medium & Medium & 100 & 0 & 0 \\
\hline Ephemeral & Low & Low & Low & High & Medium & High & 100 & 0 & 0 \\
\hline Ephemeral & Low & Low & Low & High & High & Low & 100 & 0 & 0 \\
\hline Ephemeral & Low & Low & Low & High & High & Medium & 100 & 0 & 0 \\
\hline Ephemeral & Low & Low & Low & High & High & High & 100 & 0 & 0 \\
\hline Ephemeral & Low & Low & Medium & Low & Low & Low & 100 & 0 & 0 \\
\hline Ephemeral & Low & Low & Medium & Low & Low & Medium & 100 & 0 & 0 \\
\hline Ephemeral & Low & Low & Medium & Low & Low & High & 100 & 0 & 0 \\
\hline Ephemeral & Low & Low & Medium & Low & Medium & Low & 100 & 0 & 0 \\
\hline Ephemeral & Low & Low & Medium & Low & Medium & Medium & 100 & 0 & 0 \\
\hline Ephemeral & Low & Low & Medium & Low & Medium & High & 100 & 0 & 0 \\
\hline Ephemeral & Low & Low & Medium & Low & High & Low & 100 & 0 & 0 \\
\hline
\end{tabular}




\begin{tabular}{|c|c|c|c|c|c|c|c|c|c|}
\hline \multicolumn{7}{|c|}{ Inputs } & \multicolumn{3}{|c|}{ Habitat quality } \\
\hline $\begin{array}{c}\text { Water } \\
\text { availability }\end{array}$ & Emergent & Floating & Terrestrial & $\begin{array}{l}\text { Winter } \\
\text { refuge }\end{array}$ & Submerged & $\begin{array}{l}\text { Water } \\
\text { quality }\end{array}$ & Low & Average & High \\
\hline Ephemeral & Low & Low & Medium & Low & High & Medium & 100 & 0 & 0 \\
\hline Ephemeral & Low & Low & Medium & Low & High & High & 100 & 0 & 0 \\
\hline Ephemeral & Low & Low & Medium & Medium & Low & Low & 100 & 0 & 0 \\
\hline Ephemeral & Low & Low & Medium & Medium & Low & Medium & 100 & 0 & 0 \\
\hline Ephemeral & Low & Low & Medium & Medium & Low & High & 100 & 0 & 0 \\
\hline Ephemeral & Low & Low & Medium & Medium & Medium & Low & 100 & 0 & 0 \\
\hline Ephemeral & Low & Low & Medium & Medium & Medium & Medium & 100 & 0 & 0 \\
\hline Ephemeral & Low & Low & Medium & Medium & Medium & High & 100 & 0 & 0 \\
\hline Ephemeral & Low & Low & Medium & Medium & High & Low & 100 & 0 & 0 \\
\hline Ephemeral & Low & Low & Medium & Medium & High & Medium & 100 & 0 & 0 \\
\hline Ephemeral & Low & Low & Medium & Medium & High & High & 100 & 0 & 0 \\
\hline Ephemeral & Low & Low & Medium & High & Low & Low & 100 & 0 & 0 \\
\hline Ephemeral & Low & Low & Medium & High & Low & Medium & 100 & 0 & 0 \\
\hline Ephemeral & Low & Low & Medium & High & Low & High & 100 & 0 & 0 \\
\hline Ephemeral & Low & Low & Medium & High & Medium & Low & 100 & 0 & 0 \\
\hline Ephemeral & Low & Low & Medium & High & Medium & Medium & 100 & 0 & 0 \\
\hline Ephemeral & Low & Low & Medium & High & Medium & High & 100 & 0 & 0 \\
\hline Ephemeral & Low & Low & Medium & High & High & Low & 100 & 0 & 0 \\
\hline Ephemeral & Low & Low & Medium & High & High & Medium & 100 & 0 & 0 \\
\hline Ephemeral & Low & Low & Medium & High & High & High & 100 & 0 & 0 \\
\hline Ephemeral & Low & Low & High & Low & Low & Low & 100 & 0 & 0 \\
\hline Ephemeral & Low & Low & High & Low & Low & Medium & 100 & 0 & 0 \\
\hline Ephemeral & Low & Low & High & Low & Low & High & 100 & 0 & 0 \\
\hline Ephemeral & Low & Low & High & Low & Medium & Low & 100 & 0 & 0 \\
\hline Ephemeral & Low & Low & High & Low & Medium & Medium & 100 & 0 & 0 \\
\hline Ephemeral & Low & Low & High & Low & Medium & High & 100 & 0 & 0 \\
\hline Ephemeral & Low & Low & High & Low & High & Low & 100 & 0 & 0 \\
\hline Ephemeral & Low & Low & High & Low & High & Medium & 100 & 0 & 0 \\
\hline Ephemeral & Low & Low & High & Low & High & High & 100 & 0 & 0 \\
\hline Ephemeral & Low & Low & High & Medium & Low & Low & 100 & 0 & 0 \\
\hline Ephemeral & Low & Low & High & Medium & Low & Medium & 100 & 0 & 0 \\
\hline Ephemeral & Low & Low & High & Medium & Low & High & 100 & 0 & 0 \\
\hline Ephemeral & Low & Low & High & Medium & Medium & Low & 100 & 0 & 0 \\
\hline Ephemeral & Low & Low & High & Medium & Medium & Medium & 100 & 0 & 0 \\
\hline Ephemeral & Low & Low & High & Medium & Medium & High & 100 & 0 & 0 \\
\hline Ephemeral & Low & Low & High & Medium & High & Low & 100 & 0 & 0 \\
\hline Ephemeral & Low & Low & High & Medium & High & Medium & 100 & 0 & 0 \\
\hline Ephemeral & Low & Low & High & Medium & High & High & 100 & 0 & 0 \\
\hline Ephemeral & Low & Low & High & High & Low & Low & 100 & 0 & 0 \\
\hline Ephemeral & Low & Low & High & High & Low & Medium & 100 & 0 & 0 \\
\hline
\end{tabular}




\begin{tabular}{|c|c|c|c|c|c|c|c|c|c|}
\hline \multicolumn{7}{|c|}{ Inputs } & \multicolumn{3}{|c|}{ Habitat quality } \\
\hline $\begin{array}{c}\text { Water } \\
\text { availability }\end{array}$ & Emergent & Floating & Terrestrial & $\begin{array}{l}\text { Winter } \\
\text { refuge }\end{array}$ & Submerged & $\begin{array}{l}\text { Water } \\
\text { quality }\end{array}$ & Low & Average & High \\
\hline Ephemeral & Low & Low & High & High & Low & High & 100 & 0 & 0 \\
\hline Ephemeral & Low & Low & High & High & Medium & Low & 100 & 0 & 0 \\
\hline Ephemeral & Low & Low & High & High & Medium & Medium & 100 & 0 & 0 \\
\hline Ephemeral & Low & Low & High & High & Medium & High & 100 & 0 & 0 \\
\hline Ephemeral & Low & Low & High & High & High & Low & 100 & 0 & 0 \\
\hline Ephemeral & Low & Low & High & High & High & Medium & 100 & 0 & 0 \\
\hline Ephemeral & Low & Low & High & High & High & High & 100 & 0 & 0 \\
\hline Ephemeral & Low & Medium & Low & Low & Low & Low & 100 & 0 & 0 \\
\hline Ephemeral & Low & Medium & Low & Low & Low & Medium & 100 & 0 & 0 \\
\hline Ephemeral & Low & Medium & Low & Low & Low & High & 100 & 0 & 0 \\
\hline Ephemeral & Low & Medium & Low & Low & Medium & Low & 100 & 0 & 0 \\
\hline Ephemeral & Low & Medium & Low & Low & Medium & Medium & 100 & 0 & 0 \\
\hline Ephemeral & Low & Medium & Low & Low & Medium & High & 100 & 0 & 0 \\
\hline Ephemeral & Low & Medium & Low & Low & High & Low & 100 & 0 & 0 \\
\hline Ephemeral & Low & Medium & Low & Low & High & Medium & 100 & 0 & 0 \\
\hline Ephemeral & Low & Medium & Low & Low & High & High & 100 & 0 & 0 \\
\hline Ephemeral & Low & Medium & Low & Medium & Low & Low & 100 & 0 & 0 \\
\hline Ephemeral & Low & Medium & Low & Medium & Low & Medium & 100 & 0 & 0 \\
\hline Ephemeral & Low & Medium & Low & Medium & Low & High & 100 & 0 & 0 \\
\hline Ephemeral & Low & Medium & Low & Medium & Medium & Low & 100 & 0 & 0 \\
\hline Ephemeral & Low & Medium & Low & Medium & Medium & Medium & 100 & 0 & 0 \\
\hline Ephemeral & Low & Medium & Low & Medium & Medium & High & 100 & 0 & 0 \\
\hline Ephemeral & Low & Medium & Low & Medium & High & Low & 100 & 0 & 0 \\
\hline Ephemeral & Low & Medium & Low & Medium & High & Medium & 100 & 0 & 0 \\
\hline Ephemeral & Low & Medium & Low & Medium & High & High & 100 & 0 & 0 \\
\hline Ephemeral & Low & Medium & Low & High & Low & Low & 100 & 0 & 0 \\
\hline Ephemeral & Low & Medium & Low & High & Low & Medium & 100 & 0 & 0 \\
\hline Ephemeral & Low & Medium & Low & High & Low & High & 100 & 0 & 0 \\
\hline Ephemeral & Low & Medium & Low & High & Medium & Low & 100 & 0 & 0 \\
\hline Ephemeral & Low & Medium & Low & High & Medium & Medium & 100 & 0 & 0 \\
\hline Ephemeral & Low & Medium & Low & High & Medium & High & 100 & 0 & 0 \\
\hline Ephemeral & Low & Medium & Low & High & High & Low & 100 & 0 & 0 \\
\hline Ephemeral & Low & Medium & Low & High & High & Medium & 100 & 0 & 0 \\
\hline Ephemeral & Low & Medium & Low & High & High & High & 100 & 0 & 0 \\
\hline Ephemeral & Low & Medium & Medium & Low & Low & Low & 100 & 0 & 0 \\
\hline Ephemeral & Low & Medium & Medium & Low & Low & Medium & 100 & 0 & 0 \\
\hline Ephemeral & Low & Medium & Medium & Low & Low & High & 100 & 0 & 0 \\
\hline Ephemeral & Low & Medium & Medium & Low & Medium & Low & 100 & 0 & 0 \\
\hline Ephemeral & Low & Medium & Medium & Low & Medium & Medium & 100 & 0 & 0 \\
\hline Ephemeral & Low & Medium & Medium & Low & Medium & High & 100 & 0 & 0 \\
\hline
\end{tabular}




\begin{tabular}{|c|c|c|c|c|c|c|c|c|c|}
\hline \multicolumn{7}{|c|}{ Inputs } & \multicolumn{3}{|c|}{ Habitat quality } \\
\hline $\begin{array}{c}\text { Water } \\
\text { availability }\end{array}$ & Emergent & Floating & Terrestrial & $\begin{array}{l}\text { Winter } \\
\text { refuge }\end{array}$ & Submerged & $\begin{array}{l}\text { Water } \\
\text { quality }\end{array}$ & Low & Average & High \\
\hline Ephemeral & Low & Medium & Medium & Low & High & Low & 100 & 0 & 0 \\
\hline Ephemeral & Low & Medium & Medium & Low & High & Medium & 100 & 0 & 0 \\
\hline Ephemeral & Low & Medium & Medium & Low & High & High & 100 & 0 & 0 \\
\hline Ephemeral & Low & Medium & Medium & Medium & Low & Low & 100 & 0 & 0 \\
\hline Ephemeral & Low & Medium & Medium & Medium & Low & Medium & 100 & 0 & 0 \\
\hline Ephemeral & Low & Medium & Medium & Medium & Low & High & 100 & 0 & 0 \\
\hline Ephemeral & Low & Medium & Medium & Medium & Medium & Low & 100 & 0 & 0 \\
\hline Ephemeral & Low & Medium & Medium & Medium & Medium & Medium & 100 & 0 & 0 \\
\hline Ephemeral & Low & Medium & Medium & Medium & Medium & High & 100 & 0 & 0 \\
\hline Ephemeral & Low & Medium & Medium & Medium & High & Low & 100 & 0 & 0 \\
\hline Ephemeral & Low & Medium & Medium & Medium & High & Medium & 100 & 0 & 0 \\
\hline Ephemeral & Low & Medium & Medium & Medium & High & High & 100 & 0 & 0 \\
\hline Ephemeral & Low & Medium & Medium & High & Low & Low & 100 & 0 & 0 \\
\hline Ephemeral & Low & Medium & Medium & High & Low & Medium & 100 & 0 & 0 \\
\hline Ephemeral & Low & Medium & Medium & High & Low & High & 100 & 0 & 0 \\
\hline Ephemeral & Low & Medium & Medium & High & Medium & Low & 100 & 0 & 0 \\
\hline Ephemeral & Low & Medium & Medium & High & Medium & Medium & 100 & 0 & 0 \\
\hline Ephemeral & Low & Medium & Medium & High & Medium & High & 100 & 0 & 0 \\
\hline Ephemeral & Low & Medium & Medium & High & High & Low & 100 & 0 & 0 \\
\hline Ephemeral & Low & Medium & Medium & High & High & Medium & 100 & 0 & 0 \\
\hline Ephemeral & Low & Medium & Medium & High & High & High & 100 & 0 & 0 \\
\hline Ephemeral & Low & Medium & High & Low & Low & Low & 100 & 0 & 0 \\
\hline Ephemeral & Low & Medium & High & Low & Low & Medium & 100 & 0 & 0 \\
\hline Ephemeral & Low & Medium & High & Low & Low & High & 100 & 0 & 0 \\
\hline Ephemeral & Low & Medium & High & Low & Medium & Low & 100 & 0 & 0 \\
\hline Ephemeral & Low & Medium & High & Low & Medium & Medium & 100 & 0 & 0 \\
\hline Ephemeral & Low & Medium & High & Low & Medium & High & 100 & 0 & 0 \\
\hline Ephemeral & Low & Medium & High & Low & High & Low & 100 & 0 & 0 \\
\hline Ephemeral & Low & Medium & High & Low & High & Medium & 100 & 0 & 0 \\
\hline Ephemeral & Low & Medium & High & Low & High & High & 100 & 0 & 0 \\
\hline Ephemeral & Low & Medium & High & Medium & Low & Low & 100 & 0 & 0 \\
\hline Ephemeral & Low & Medium & High & Medium & Low & Medium & 100 & 0 & 0 \\
\hline Ephemeral & Low & Medium & High & Medium & Low & High & 100 & 0 & 0 \\
\hline Ephemeral & Low & Medium & High & Medium & Medium & Low & 100 & 0 & 0 \\
\hline Ephemeral & Low & Medium & High & Medium & Medium & Medium & 100 & 0 & 0 \\
\hline Ephemeral & Low & Medium & High & Medium & Medium & High & 100 & 0 & 0 \\
\hline Ephemeral & Low & Medium & High & Medium & High & Low & 100 & 0 & 0 \\
\hline Ephemeral & Low & Medium & High & Medium & High & Medium & 100 & 0 & 0 \\
\hline Ephemeral & Low & Medium & High & Medium & High & High & 100 & 0 & 0 \\
\hline Ephemeral & Low & Medium & High & High & Low & Low & 100 & 0 & 0 \\
\hline
\end{tabular}




\begin{tabular}{|c|c|c|c|c|c|c|c|c|c|}
\hline \multicolumn{7}{|c|}{ Inputs } & \multicolumn{3}{|c|}{ Habitat quality } \\
\hline $\begin{array}{c}\text { Water } \\
\text { availability }\end{array}$ & Emergent & Floating & Terrestrial & $\begin{array}{l}\text { Winter } \\
\text { refuge }\end{array}$ & Submerged & $\begin{array}{l}\text { Water } \\
\text { quality }\end{array}$ & Low & Average & High \\
\hline Ephemeral & Low & Medium & High & High & Low & Medium & 100 & 0 & 0 \\
\hline Ephemeral & Low & Medium & High & High & Low & High & 100 & 0 & 0 \\
\hline Ephemeral & Low & Medium & High & High & Medium & Low & 100 & 0 & 0 \\
\hline Ephemeral & Low & Medium & High & High & Medium & Medium & 100 & 0 & 0 \\
\hline Ephemeral & Low & Medium & High & High & Medium & High & 100 & 0 & 0 \\
\hline Ephemeral & Low & Medium & High & High & High & Low & 100 & 0 & 0 \\
\hline Ephemeral & Low & Medium & High & High & High & Medium & 100 & 0 & 0 \\
\hline Ephemeral & Low & Medium & High & High & High & High & 100 & 0 & 0 \\
\hline Ephemeral & Low & High & Low & Low & Low & Low & 100 & 0 & 0 \\
\hline Ephemeral & Low & High & Low & Low & Low & Medium & 100 & 0 & 0 \\
\hline Ephemeral & Low & High & Low & Low & Low & High & 100 & 0 & 0 \\
\hline Ephemeral & Low & High & Low & Low & Medium & Low & 100 & 0 & 0 \\
\hline Ephemeral & Low & High & Low & Low & Medium & Medium & 100 & 0 & 0 \\
\hline Ephemeral & Low & High & Low & Low & Medium & High & 100 & 0 & 0 \\
\hline Ephemeral & Low & High & Low & Low & High & Low & 100 & 0 & 0 \\
\hline Ephemeral & Low & High & Low & Low & High & Medium & 100 & 0 & 0 \\
\hline Ephemeral & Low & High & Low & Low & High & High & 100 & 0 & 0 \\
\hline Ephemeral & Low & High & Low & Medium & Low & Low & 100 & 0 & 0 \\
\hline Ephemeral & Low & High & Low & Medium & Low & Medium & 100 & 0 & 0 \\
\hline Ephemeral & Low & High & Low & Medium & Low & High & 100 & 0 & 0 \\
\hline Ephemeral & Low & High & Low & Medium & Medium & Low & 100 & 0 & 0 \\
\hline Ephemeral & Low & High & Low & Medium & Medium & Medium & 100 & 0 & 0 \\
\hline Ephemeral & Low & High & Low & Medium & Medium & High & 100 & 0 & 0 \\
\hline Ephemeral & Low & High & Low & Medium & High & Low & 100 & 0 & 0 \\
\hline Ephemeral & Low & High & Low & Medium & High & Medium & 100 & 0 & 0 \\
\hline Ephemeral & Low & High & Low & Medium & High & High & 100 & 0 & 0 \\
\hline Ephemeral & Low & High & Low & High & Low & Low & 100 & 0 & 0 \\
\hline Ephemeral & Low & High & Low & High & Low & Medium & 100 & 0 & 0 \\
\hline Ephemeral & Low & High & Low & High & Low & High & 100 & 0 & 0 \\
\hline Ephemeral & Low & High & Low & High & Medium & Low & 100 & 0 & 0 \\
\hline Ephemeral & Low & High & Low & High & Medium & Medium & 100 & 0 & 0 \\
\hline Ephemeral & Low & High & Low & High & Medium & High & 100 & 0 & 0 \\
\hline Ephemeral & Low & High & Low & High & High & Low & 100 & 0 & 0 \\
\hline Ephemeral & Low & High & Low & High & High & Medium & 100 & 0 & 0 \\
\hline Ephemeral & Low & High & Low & High & High & High & 100 & 0 & 0 \\
\hline Ephemeral & Low & High & Medium & Low & Low & Low & 100 & 0 & 0 \\
\hline Ephemeral & Low & High & Medium & Low & Low & Medium & 100 & 0 & 0 \\
\hline Ephemeral & Low & High & Medium & Low & Low & High & 100 & 0 & 0 \\
\hline Ephemeral & Low & High & Medium & Low & Medium & Low & 100 & 0 & 0 \\
\hline Ephemeral & Low & High & Medium & Low & Medium & Medium & 100 & 0 & 0 \\
\hline
\end{tabular}




\begin{tabular}{|c|c|c|c|c|c|c|c|c|c|}
\hline \multicolumn{7}{|c|}{ Inputs } & \multicolumn{3}{|c|}{ Habitat quality } \\
\hline $\begin{array}{c}\text { Water } \\
\text { availability }\end{array}$ & Emergent & Floating & Terrestrial & $\begin{array}{l}\text { Winter } \\
\text { refuge }\end{array}$ & Submerged & $\begin{array}{l}\text { Water } \\
\text { quality }\end{array}$ & Low & Average & High \\
\hline Ephemeral & Low & High & Medium & Low & Medium & High & 100 & 0 & 0 \\
\hline Ephemeral & Low & High & Medium & Low & High & Low & 100 & 0 & 0 \\
\hline Ephemeral & Low & High & Medium & Low & High & Medium & 100 & 0 & 0 \\
\hline Ephemeral & Low & High & Medium & Low & High & High & 100 & 0 & 0 \\
\hline Ephemeral & Low & High & Medium & Medium & Low & Low & 100 & 0 & 0 \\
\hline Ephemeral & Low & High & Medium & Medium & Low & Medium & 100 & 0 & 0 \\
\hline Ephemeral & Low & High & Medium & Medium & Low & High & 100 & 0 & 0 \\
\hline Ephemeral & Low & High & Medium & Medium & Medium & Low & 100 & 0 & 0 \\
\hline Ephemeral & Low & High & Medium & Medium & Medium & Medium & 100 & 0 & 0 \\
\hline Ephemeral & Low & High & Medium & Medium & Medium & High & 100 & 0 & 0 \\
\hline Ephemeral & Low & High & Medium & Medium & High & Low & 100 & 0 & 0 \\
\hline Ephemeral & Low & High & Medium & Medium & High & Medium & 100 & 0 & 0 \\
\hline Ephemeral & Low & High & Medium & Medium & High & High & 100 & 0 & 0 \\
\hline Ephemeral & Low & High & Medium & High & Low & Low & 100 & 0 & 0 \\
\hline Ephemeral & Low & High & Medium & High & Low & Medium & 100 & 0 & 0 \\
\hline Ephemeral & Low & High & Medium & High & Low & High & 100 & 0 & 0 \\
\hline Ephemeral & Low & High & Medium & High & Medium & Low & 100 & 0 & 0 \\
\hline Ephemeral & Low & High & Medium & High & Medium & Medium & 100 & 0 & 0 \\
\hline Ephemeral & Low & High & Medium & High & Medium & High & 100 & 0 & 0 \\
\hline Ephemeral & Low & High & Medium & High & High & Low & 100 & 0 & 0 \\
\hline Ephemeral & Low & High & Medium & High & High & Medium & 100 & 0 & 0 \\
\hline Ephemeral & Low & High & Medium & High & High & High & 100 & 0 & 0 \\
\hline Ephemeral & Low & High & High & Low & Low & Low & 100 & 0 & 0 \\
\hline Ephemeral & Low & High & High & Low & Low & Medium & 100 & 0 & 0 \\
\hline Ephemeral & Low & High & High & Low & Low & High & 100 & 0 & 0 \\
\hline Ephemeral & Low & High & High & Low & Medium & Low & 100 & 0 & 0 \\
\hline Ephemeral & Low & High & High & Low & Medium & Medium & 100 & 0 & 0 \\
\hline Ephemeral & Low & High & High & Low & Medium & High & 100 & 0 & 0 \\
\hline Ephemeral & Low & High & High & Low & High & Low & 100 & 0 & 0 \\
\hline Ephemeral & Low & High & High & Low & High & Medium & 100 & 0 & 0 \\
\hline Ephemeral & Low & High & High & Low & High & High & 100 & 0 & 0 \\
\hline Ephemeral & Low & High & High & Medium & Low & Low & 100 & 0 & 0 \\
\hline Ephemeral & Low & High & High & Medium & Low & Medium & 100 & 0 & 0 \\
\hline Ephemeral & Low & High & High & Medium & Low & High & 100 & 0 & 0 \\
\hline Ephemeral & Low & High & High & Medium & Medium & Low & 100 & 0 & 0 \\
\hline Ephemeral & Low & High & High & Medium & Medium & Medium & 100 & 0 & 0 \\
\hline Ephemeral & Low & High & High & Medium & Medium & High & 100 & 0 & 0 \\
\hline Ephemeral & Low & High & High & Medium & High & Low & 100 & 0 & 0 \\
\hline Ephemeral & Low & High & High & Medium & High & Medium & 100 & 0 & 0 \\
\hline Ephemeral & Low & High & High & Medium & High & High & 100 & 0 & 0 \\
\hline
\end{tabular}




\begin{tabular}{|c|c|c|c|c|c|c|c|c|c|}
\hline \multicolumn{7}{|c|}{ Inputs } & \multicolumn{3}{|c|}{ Habitat quality } \\
\hline $\begin{array}{c}\text { Water } \\
\text { availability }\end{array}$ & Emergent & Floating & Terrestrial & $\begin{array}{l}\text { Winter } \\
\text { refuge }\end{array}$ & Submerged & $\begin{array}{l}\text { Water } \\
\text { quality }\end{array}$ & Low & Average & High \\
\hline Ephemeral & Low & High & High & High & Low & Low & 100 & 0 & 0 \\
\hline Ephemeral & Low & High & High & High & Low & Medium & 100 & 0 & 0 \\
\hline Ephemeral & Low & High & High & High & Low & High & 100 & 0 & 0 \\
\hline Ephemeral & Low & High & High & High & Medium & Low & 100 & 0 & 0 \\
\hline Ephemeral & Low & High & High & High & Medium & Medium & 100 & 0 & 0 \\
\hline Ephemeral & Low & High & High & High & Medium & High & 100 & 0 & 0 \\
\hline Ephemeral & Low & High & High & High & High & Low & 100 & 0 & 0 \\
\hline Ephemeral & Low & High & High & High & High & Medium & 100 & 0 & 0 \\
\hline Ephemeral & Low & High & High & High & High & High & 100 & 0 & 0 \\
\hline Ephemeral & Medium & Low & Low & Low & Low & Low & 100 & 0 & 0 \\
\hline Ephemeral & Medium & Low & Low & Low & Low & Medium & 100 & 0 & 0 \\
\hline Ephemeral & Medium & Low & Low & Low & Low & High & 100 & 0 & 0 \\
\hline Ephemeral & Medium & Low & Low & Low & Medium & Low & 100 & 0 & 0 \\
\hline Ephemeral & Medium & Low & Low & Low & Medium & Medium & 100 & 0 & 0 \\
\hline Ephemeral & Medium & Low & Low & Low & Medium & High & 100 & 0 & 0 \\
\hline Ephemeral & Medium & Low & Low & Low & High & Low & 100 & 0 & 0 \\
\hline Ephemeral & Medium & Low & Low & Low & High & Medium & 100 & 0 & 0 \\
\hline Ephemeral & Medium & Low & Low & Low & High & High & 100 & 0 & 0 \\
\hline Ephemeral & Medium & Low & Low & Medium & Low & Low & 100 & 0 & 0 \\
\hline Ephemeral & Medium & Low & Low & Medium & Low & Medium & 100 & 0 & 0 \\
\hline Ephemeral & Medium & Low & Low & Medium & Low & High & 100 & 0 & 0 \\
\hline Ephemeral & Medium & Low & Low & Medium & Medium & Low & 100 & 0 & 0 \\
\hline Ephemeral & Medium & Low & Low & Medium & Medium & Medium & 100 & 0 & 0 \\
\hline Ephemeral & Medium & Low & Low & Medium & Medium & High & 100 & 0 & 0 \\
\hline Ephemeral & Medium & Low & Low & Medium & High & Low & 100 & 0 & 0 \\
\hline Ephemeral & Medium & Low & Low & Medium & High & Medium & 100 & 0 & 0 \\
\hline Ephemeral & Medium & Low & Low & Medium & High & High & 100 & 0 & 0 \\
\hline Ephemeral & Medium & Low & Low & High & Low & Low & 100 & 0 & 0 \\
\hline Ephemeral & Medium & Low & Low & High & Low & Medium & 100 & 0 & 0 \\
\hline Ephemeral & Medium & Low & Low & High & Low & High & 100 & 0 & 0 \\
\hline Ephemeral & Medium & Low & Low & High & Medium & Low & 100 & 0 & 0 \\
\hline Ephemeral & Medium & Low & Low & High & Medium & Medium & 100 & 0 & 0 \\
\hline Ephemeral & Medium & Low & Low & High & Medium & High & 100 & 0 & 0 \\
\hline Ephemeral & Medium & Low & Low & High & High & Low & 100 & 0 & 0 \\
\hline Ephemeral & Medium & Low & Low & High & High & Medium & 100 & 0 & 0 \\
\hline Ephemeral & Medium & Low & Low & High & High & High & 100 & 0 & 0 \\
\hline Ephemeral & Medium & Low & Medium & Low & Low & Low & 100 & 0 & 0 \\
\hline Ephemeral & Medium & Low & Medium & Low & Low & Medium & 100 & 0 & 0 \\
\hline Ephemeral & Medium & Low & Medium & Low & Low & High & 100 & 0 & 0 \\
\hline Ephemeral & Medium & Low & Medium & Low & Medium & Low & 100 & 0 & 0 \\
\hline
\end{tabular}




\begin{tabular}{|c|c|c|c|c|c|c|c|c|c|}
\hline \multicolumn{7}{|c|}{ Inputs } & \multicolumn{3}{|c|}{ Habitat quality } \\
\hline $\begin{array}{c}\text { Water } \\
\text { availability }\end{array}$ & Emergent & Floating & Terrestrial & $\begin{array}{l}\text { Winter } \\
\text { refuge }\end{array}$ & Submerged & $\begin{array}{l}\text { Water } \\
\text { quality }\end{array}$ & Low & Average & High \\
\hline Ephemeral & Medium & Low & Medium & Low & Medium & Medium & 100 & 0 & 0 \\
\hline Ephemeral & Medium & Low & Medium & Low & Medium & High & 100 & 0 & 0 \\
\hline Ephemeral & Medium & Low & Medium & Low & High & Low & 100 & 0 & 0 \\
\hline Ephemeral & Medium & Low & Medium & Low & High & Medium & 100 & 0 & 0 \\
\hline Ephemeral & Medium & Low & Medium & Low & High & High & 100 & 0 & 0 \\
\hline Ephemeral & Medium & Low & Medium & Medium & Low & Low & 100 & 0 & 0 \\
\hline Ephemeral & Medium & Low & Medium & Medium & Low & Medium & 100 & 0 & 0 \\
\hline Ephemeral & Medium & Low & Medium & Medium & Low & High & 100 & 0 & 0 \\
\hline Ephemeral & Medium & Low & Medium & Medium & Medium & Low & 100 & 0 & 0 \\
\hline Ephemeral & Medium & Low & Medium & Medium & Medium & Medium & 100 & 0 & 0 \\
\hline Ephemeral & Medium & Low & Medium & Medium & Medium & High & 100 & 0 & 0 \\
\hline Ephemeral & Medium & Low & Medium & Medium & High & Low & 100 & 0 & 0 \\
\hline Ephemeral & Medium & Low & Medium & Medium & High & Medium & 100 & 0 & 0 \\
\hline Ephemeral & Medium & Low & Medium & Medium & High & High & 100 & 0 & 0 \\
\hline Ephemeral & Medium & Low & Medium & High & Low & Low & 100 & 0 & 0 \\
\hline Ephemeral & Medium & Low & Medium & High & Low & Medium & 100 & 0 & 0 \\
\hline Ephemeral & Medium & Low & Medium & High & Low & High & 100 & 0 & 0 \\
\hline Ephemeral & Medium & Low & Medium & High & Medium & Low & 100 & 0 & 0 \\
\hline Ephemeral & Medium & Low & Medium & High & Medium & Medium & 100 & 0 & 0 \\
\hline Ephemeral & Medium & Low & Medium & High & Medium & High & 100 & 0 & 0 \\
\hline Ephemeral & Medium & Low & Medium & High & High & Low & 100 & 0 & 0 \\
\hline Ephemeral & Medium & Low & Medium & High & High & Medium & 100 & 0 & 0 \\
\hline Ephemeral & Medium & Low & Medium & High & High & High & 100 & 0 & 0 \\
\hline Ephemeral & Medium & Low & High & Low & Low & Low & 100 & 0 & 0 \\
\hline Ephemeral & Medium & Low & High & Low & Low & Medium & 100 & 0 & 0 \\
\hline Ephemeral & Medium & Low & High & Low & Low & High & 100 & 0 & 0 \\
\hline Ephemeral & Medium & Low & High & Low & Medium & Low & 100 & 0 & 0 \\
\hline Ephemeral & Medium & Low & High & Low & Medium & Medium & 100 & 0 & 0 \\
\hline Ephemeral & Medium & Low & High & Low & Medium & High & 100 & 0 & 0 \\
\hline Ephemeral & Medium & Low & High & Low & High & Low & 100 & 0 & 0 \\
\hline Ephemeral & Medium & Low & High & Low & High & Medium & 100 & 0 & 0 \\
\hline Ephemeral & Medium & Low & High & Low & High & High & 100 & 0 & 0 \\
\hline Ephemeral & Medium & Low & High & Medium & Low & Low & 100 & 0 & 0 \\
\hline Ephemeral & Medium & Low & High & Medium & Low & Medium & 100 & 0 & 0 \\
\hline Ephemeral & Medium & Low & High & Medium & Low & High & 100 & 0 & 0 \\
\hline Ephemeral & Medium & Low & High & Medium & Medium & Low & 100 & 0 & 0 \\
\hline Ephemeral & Medium & Low & High & Medium & Medium & Medium & 100 & 0 & 0 \\
\hline Ephemeral & Medium & Low & High & Medium & Medium & High & 100 & 0 & 0 \\
\hline Ephemeral & Medium & Low & High & Medium & High & Low & 100 & 0 & 0 \\
\hline Ephemeral & Medium & Low & High & Medium & High & Medium & 100 & 0 & 0 \\
\hline
\end{tabular}




\begin{tabular}{|c|c|c|c|c|c|c|c|c|c|}
\hline \multicolumn{7}{|c|}{ Inputs } & \multicolumn{3}{|c|}{ Habitat quality } \\
\hline $\begin{array}{c}\text { Water } \\
\text { availability }\end{array}$ & Emergent & Floating & Terrestrial & $\begin{array}{l}\text { Winter } \\
\text { refuge }\end{array}$ & Submerged & $\begin{array}{l}\text { Water } \\
\text { quality }\end{array}$ & Low & Average & High \\
\hline Ephemeral & Medium & Low & High & Medium & High & High & 100 & 0 & 0 \\
\hline Ephemeral & Medium & Low & High & High & Low & Low & 100 & 0 & 0 \\
\hline Ephemeral & Medium & Low & High & High & Low & Medium & 100 & 0 & 0 \\
\hline Ephemeral & Medium & Low & High & High & Low & High & 100 & 0 & 0 \\
\hline Ephemeral & Medium & Low & High & High & Medium & Low & 100 & 0 & 0 \\
\hline Ephemeral & Medium & Low & High & High & Medium & Medium & 100 & 0 & 0 \\
\hline Ephemeral & Medium & Low & High & High & Medium & High & 100 & 0 & 0 \\
\hline Ephemeral & Medium & Low & High & High & High & Low & 100 & 0 & 0 \\
\hline Ephemeral & Medium & Low & High & High & High & Medium & 100 & 0 & 0 \\
\hline Ephemeral & Medium & Low & High & High & High & High & 100 & 0 & 0 \\
\hline Ephemeral & Medium & Medium & Low & Low & Low & Low & 100 & 0 & 0 \\
\hline Ephemeral & Medium & Medium & Low & Low & Low & Medium & 100 & 0 & 0 \\
\hline Ephemeral & Medium & Medium & Low & Low & Low & High & 100 & 0 & 0 \\
\hline Ephemeral & Medium & Medium & Low & Low & Medium & Low & 100 & 0 & 0 \\
\hline Ephemeral & Medium & Medium & Low & Low & Medium & Medium & 100 & 0 & 0 \\
\hline Ephemeral & Medium & Medium & Low & Low & Medium & High & 100 & 0 & 0 \\
\hline Ephemeral & Medium & Medium & Low & Low & High & Low & 100 & 0 & 0 \\
\hline Ephemeral & Medium & Medium & Low & Low & High & Medium & 100 & 0 & 0 \\
\hline Ephemeral & Medium & Medium & Low & Low & High & High & 100 & 0 & 0 \\
\hline Ephemeral & Medium & Medium & Low & Medium & Low & Low & 100 & 0 & 0 \\
\hline Ephemeral & Medium & Medium & Low & Medium & Low & Medium & 100 & 0 & 0 \\
\hline Ephemeral & Medium & Medium & Low & Medium & Low & High & 100 & 0 & 0 \\
\hline Ephemeral & Medium & Medium & Low & Medium & Medium & Low & 100 & 0 & 0 \\
\hline Ephemeral & Medium & Medium & Low & Medium & Medium & Medium & 100 & 0 & 0 \\
\hline Ephemeral & Medium & Medium & Low & Medium & Medium & High & 100 & 0 & 0 \\
\hline Ephemeral & Medium & Medium & Low & Medium & High & Low & 100 & 0 & 0 \\
\hline Ephemeral & Medium & Medium & Low & Medium & High & Medium & 100 & 0 & 0 \\
\hline Ephemeral & Medium & Medium & Low & Medium & High & High & 100 & 0 & 0 \\
\hline Ephemeral & Medium & Medium & Low & High & Low & Low & 100 & 0 & 0 \\
\hline Ephemeral & Medium & Medium & Low & High & Low & Medium & 100 & 0 & 0 \\
\hline Ephemeral & Medium & Medium & Low & High & Low & High & 100 & 0 & 0 \\
\hline Ephemeral & Medium & Medium & Low & High & Medium & Low & 100 & 0 & 0 \\
\hline Ephemeral & Medium & Medium & Low & High & Medium & Medium & 100 & 0 & 0 \\
\hline Ephemeral & Medium & Medium & Low & High & Medium & High & 100 & 0 & 0 \\
\hline Ephemeral & Medium & Medium & Low & High & High & Low & 100 & 0 & 0 \\
\hline Ephemeral & Medium & Medium & Low & High & High & Medium & 100 & 0 & 0 \\
\hline Ephemeral & Medium & Medium & Low & High & High & High & 100 & 0 & 0 \\
\hline Ephemeral & Medium & Medium & Medium & Low & Low & Low & 100 & 0 & 0 \\
\hline Ephemeral & Medium & Medium & Medium & Low & Low & Medium & 100 & 0 & 0 \\
\hline Ephemeral & Medium & Medium & Medium & Low & Low & High & 100 & 0 & 0 \\
\hline
\end{tabular}




\begin{tabular}{|c|c|c|c|c|c|c|c|c|c|}
\hline \multicolumn{7}{|c|}{ Inputs } & \multicolumn{3}{|c|}{ Habitat quality } \\
\hline $\begin{array}{c}\text { Water } \\
\text { availability }\end{array}$ & Emergent & Floating & Terrestrial & $\begin{array}{l}\text { Winter } \\
\text { refuge }\end{array}$ & Submerged & $\begin{array}{l}\text { Water } \\
\text { quality }\end{array}$ & Low & Average & High \\
\hline Ephemeral & Medium & Medium & Medium & Low & Medium & Low & 100 & 0 & 0 \\
\hline Ephemeral & Medium & Medium & Medium & Low & Medium & Medium & 100 & 0 & 0 \\
\hline Ephemeral & Medium & Medium & Medium & Low & Medium & High & 100 & 0 & 0 \\
\hline Ephemeral & Medium & Medium & Medium & Low & High & Low & 100 & 0 & 0 \\
\hline Ephemeral & Medium & Medium & Medium & Low & High & Medium & 100 & 0 & 0 \\
\hline Ephemeral & Medium & Medium & Medium & Low & High & High & 100 & 0 & 0 \\
\hline Ephemeral & Medium & Medium & Medium & Medium & Low & Low & 100 & 0 & 0 \\
\hline Ephemeral & Medium & Medium & Medium & Medium & Low & Medium & 100 & 0 & 0 \\
\hline Ephemeral & Medium & Medium & Medium & Medium & Low & High & 100 & 0 & 0 \\
\hline Ephemeral & Medium & Medium & Medium & Medium & Medium & Low & 100 & 0 & 0 \\
\hline Ephemeral & Medium & Medium & Medium & Medium & Medium & Medium & 100 & 0 & 0 \\
\hline Ephemeral & Medium & Medium & Medium & Medium & Medium & High & 100 & 0 & 0 \\
\hline Ephemeral & Medium & Medium & Medium & Medium & High & Low & 100 & 0 & 0 \\
\hline Ephemeral & Medium & Medium & Medium & Medium & High & Medium & 100 & 0 & 0 \\
\hline Ephemeral & Medium & Medium & Medium & Medium & High & High & 100 & 0 & 0 \\
\hline Ephemeral & Medium & Medium & Medium & High & Low & Low & 100 & 0 & 0 \\
\hline Ephemeral & Medium & Medium & Medium & High & Low & Medium & 100 & 0 & 0 \\
\hline Ephemeral & Medium & Medium & Medium & High & Low & High & 100 & 0 & 0 \\
\hline Ephemeral & Medium & Medium & Medium & High & Medium & Low & 100 & 0 & 0 \\
\hline Ephemeral & Medium & Medium & Medium & High & Medium & Medium & 100 & 0 & 0 \\
\hline Ephemeral & Medium & Medium & Medium & High & Medium & High & 100 & 0 & 0 \\
\hline Ephemeral & Medium & Medium & Medium & High & High & Low & 100 & 0 & 0 \\
\hline Ephemeral & Medium & Medium & Medium & High & High & Medium & 100 & 0 & 0 \\
\hline Ephemeral & Medium & Medium & Medium & High & High & High & 100 & 0 & 0 \\
\hline Ephemeral & Medium & Medium & High & Low & Low & Low & 100 & 0 & 0 \\
\hline Ephemeral & Medium & Medium & High & Low & Low & Medium & 100 & 0 & 0 \\
\hline Ephemeral & Medium & Medium & High & Low & Low & High & 100 & 0 & 0 \\
\hline Ephemeral & Medium & Medium & High & Low & Medium & Low & 100 & 0 & 0 \\
\hline Ephemeral & Medium & Medium & High & Low & Medium & Medium & 100 & 0 & 0 \\
\hline Ephemeral & Medium & Medium & High & Low & Medium & High & 100 & 0 & 0 \\
\hline Ephemeral & Medium & Medium & High & Low & High & Low & 100 & 0 & 0 \\
\hline Ephemeral & Medium & Medium & High & Low & High & Medium & 100 & 0 & 0 \\
\hline Ephemeral & Medium & Medium & High & Low & High & High & 100 & 0 & 0 \\
\hline Ephemeral & Medium & Medium & High & Medium & Low & Low & 100 & 0 & 0 \\
\hline Ephemeral & Medium & Medium & High & Medium & Low & Medium & 100 & 0 & 0 \\
\hline Ephemeral & Medium & Medium & High & Medium & Low & High & 100 & 0 & 0 \\
\hline Ephemeral & Medium & Medium & High & Medium & Medium & Low & 100 & 0 & 0 \\
\hline Ephemeral & Medium & Medium & High & Medium & Medium & Medium & 100 & 0 & 0 \\
\hline Ephemeral & Medium & Medium & High & Medium & Medium & High & 100 & 0 & 0 \\
\hline Ephemeral & Medium & Medium & High & Medium & High & Low & 100 & 0 & 0 \\
\hline
\end{tabular}




\begin{tabular}{|c|c|c|c|c|c|c|c|c|c|}
\hline \multicolumn{7}{|c|}{ Inputs } & \multicolumn{3}{|c|}{ Habitat quality } \\
\hline $\begin{array}{c}\text { Water } \\
\text { availability }\end{array}$ & Emergent & Floating & Terrestrial & $\begin{array}{l}\text { Winter } \\
\text { refuge }\end{array}$ & Submerged & $\begin{array}{l}\text { Water } \\
\text { quality }\end{array}$ & Low & Average & High \\
\hline Ephemeral & Medium & Medium & High & Medium & High & Medium & 100 & 0 & 0 \\
\hline Ephemeral & Medium & Medium & High & Medium & High & High & 100 & 0 & 0 \\
\hline Ephemeral & Medium & Medium & High & High & Low & Low & 100 & 0 & 0 \\
\hline Ephemeral & Medium & Medium & High & High & Low & Medium & 100 & 0 & 0 \\
\hline Ephemeral & Medium & Medium & High & High & Low & High & 100 & 0 & 0 \\
\hline Ephemeral & Medium & Medium & High & High & Medium & Low & 100 & 0 & 0 \\
\hline Ephemeral & Medium & Medium & High & High & Medium & Medium & 100 & 0 & 0 \\
\hline Ephemeral & Medium & Medium & High & High & Medium & High & 100 & 0 & 0 \\
\hline Ephemeral & Medium & Medium & High & High & High & Low & 100 & 0 & 0 \\
\hline Ephemeral & Medium & Medium & High & High & High & Medium & 100 & 0 & 0 \\
\hline Ephemeral & Medium & Medium & High & High & High & High & 100 & 0 & 0 \\
\hline Ephemeral & Medium & High & Low & Low & Low & Low & 100 & 0 & 0 \\
\hline Ephemeral & Medium & High & Low & Low & Low & Medium & 100 & 0 & 0 \\
\hline Ephemeral & Medium & High & Low & Low & Low & High & 100 & 0 & 0 \\
\hline Ephemeral & Medium & High & Low & Low & Medium & Low & 100 & 0 & 0 \\
\hline Ephemeral & Medium & High & Low & Low & Medium & Medium & 100 & 0 & 0 \\
\hline Ephemeral & Medium & High & Low & Low & Medium & High & 100 & 0 & 0 \\
\hline Ephemeral & Medium & High & Low & Low & High & Low & 100 & 0 & 0 \\
\hline Ephemeral & Medium & High & Low & Low & High & Medium & 100 & 0 & 0 \\
\hline Ephemeral & Medium & High & Low & Low & High & High & 100 & 0 & 0 \\
\hline Ephemeral & Medium & High & Low & Medium & Low & Low & 100 & 0 & 0 \\
\hline Ephemeral & Medium & High & Low & Medium & Low & Medium & 100 & 0 & 0 \\
\hline Ephemeral & Medium & High & Low & Medium & Low & High & 100 & 0 & 0 \\
\hline Ephemeral & Medium & High & Low & Medium & Medium & Low & 100 & 0 & 0 \\
\hline Ephemeral & Medium & High & Low & Medium & Medium & Medium & 100 & 0 & 0 \\
\hline Ephemeral & Medium & High & Low & Medium & Medium & High & 100 & 0 & 0 \\
\hline Ephemeral & Medium & High & Low & Medium & High & Low & 100 & 0 & 0 \\
\hline Ephemeral & Medium & High & Low & Medium & High & Medium & 100 & 0 & 0 \\
\hline Ephemeral & Medium & High & Low & Medium & High & High & 100 & 0 & 0 \\
\hline Ephemeral & Medium & High & Low & High & Low & Low & 100 & 0 & 0 \\
\hline Ephemeral & Medium & High & Low & High & Low & Medium & 100 & 0 & 0 \\
\hline Ephemeral & Medium & High & Low & High & Low & High & 100 & 0 & 0 \\
\hline Ephemeral & Medium & High & Low & High & Medium & Low & 100 & 0 & 0 \\
\hline Ephemeral & Medium & High & Low & High & Medium & Medium & 100 & 0 & 0 \\
\hline Ephemeral & Medium & High & Low & High & Medium & High & 100 & 0 & 0 \\
\hline Ephemeral & Medium & High & Low & High & High & Low & 100 & 0 & 0 \\
\hline Ephemeral & Medium & High & Low & High & High & Medium & 100 & 0 & 0 \\
\hline Ephemeral & Medium & High & Low & High & High & High & 100 & 0 & 0 \\
\hline Ephemeral & Medium & High & Medium & Low & Low & Low & 100 & 0 & 0 \\
\hline Ephemeral & Medium & High & Medium & Low & Low & Medium & 100 & 0 & 0 \\
\hline
\end{tabular}




\begin{tabular}{|c|c|c|c|c|c|c|c|c|c|}
\hline \multicolumn{7}{|c|}{ Inputs } & \multicolumn{3}{|c|}{ Habitat quality } \\
\hline $\begin{array}{c}\text { Water } \\
\text { availability }\end{array}$ & Emergent & Floating & Terrestrial & $\begin{array}{l}\text { Winter } \\
\text { refuge }\end{array}$ & Submerged & $\begin{array}{l}\text { Water } \\
\text { quality }\end{array}$ & Low & Average & High \\
\hline Ephemeral & Medium & High & Medium & Low & Low & High & 100 & 0 & 0 \\
\hline Ephemeral & Medium & High & Medium & Low & Medium & Low & 100 & 0 & 0 \\
\hline Ephemeral & Medium & High & Medium & Low & Medium & Medium & 100 & 0 & 0 \\
\hline Ephemeral & Medium & High & Medium & Low & Medium & High & 100 & 0 & 0 \\
\hline Ephemeral & Medium & High & Medium & Low & High & Low & 100 & 0 & 0 \\
\hline Ephemeral & Medium & High & Medium & Low & High & Medium & 100 & 0 & 0 \\
\hline Ephemeral & Medium & High & Medium & Low & High & High & 100 & 0 & 0 \\
\hline Ephemeral & Medium & High & Medium & Medium & Low & Low & 100 & 0 & 0 \\
\hline Ephemeral & Medium & High & Medium & Medium & Low & Medium & 100 & 0 & 0 \\
\hline Ephemeral & Medium & High & Medium & Medium & Low & High & 100 & 0 & 0 \\
\hline Ephemeral & Medium & High & Medium & Medium & Medium & Low & 100 & 0 & 0 \\
\hline Ephemeral & Medium & High & Medium & Medium & Medium & Medium & 100 & 0 & 0 \\
\hline Ephemeral & Medium & High & Medium & Medium & Medium & High & 100 & 0 & 0 \\
\hline Ephemeral & Medium & High & Medium & Medium & High & Low & 100 & 0 & 0 \\
\hline Ephemeral & Medium & High & Medium & Medium & High & Medium & 100 & 0 & 0 \\
\hline Ephemeral & Medium & High & Medium & Medium & High & High & 100 & 0 & 0 \\
\hline Ephemeral & Medium & High & Medium & High & Low & Low & 100 & 0 & 0 \\
\hline Ephemeral & Medium & High & Medium & High & Low & Medium & 100 & 0 & 0 \\
\hline Ephemeral & Medium & High & Medium & High & Low & High & 100 & 0 & 0 \\
\hline Ephemeral & Medium & High & Medium & High & Medium & Low & 100 & 0 & 0 \\
\hline Ephemeral & Medium & High & Medium & High & Medium & Medium & 100 & 0 & 0 \\
\hline Ephemeral & Medium & High & Medium & High & Medium & High & 100 & 0 & 0 \\
\hline Ephemeral & Medium & High & Medium & High & High & Low & 100 & 0 & 0 \\
\hline Ephemeral & Medium & High & Medium & High & High & Medium & 100 & 0 & 0 \\
\hline Ephemeral & Medium & High & Medium & High & High & High & 100 & 0 & 0 \\
\hline Ephemeral & Medium & High & High & Low & Low & Low & 100 & 0 & 0 \\
\hline Ephemeral & Medium & High & High & Low & Low & Medium & 100 & 0 & 0 \\
\hline Ephemeral & Medium & High & High & Low & Low & High & 100 & 0 & 0 \\
\hline Ephemeral & Medium & High & High & Low & Medium & Low & 100 & 0 & 0 \\
\hline Ephemeral & Medium & High & High & Low & Medium & Medium & 100 & 0 & 0 \\
\hline Ephemeral & Medium & High & High & Low & Medium & High & 100 & 0 & 0 \\
\hline Ephemeral & Medium & High & High & Low & High & Low & 100 & 0 & 0 \\
\hline Ephemeral & Medium & High & High & Low & High & Medium & 100 & 0 & 0 \\
\hline Ephemeral & Medium & High & High & Low & High & High & 100 & 0 & 0 \\
\hline Ephemeral & Medium & High & High & Medium & Low & Low & 100 & 0 & 0 \\
\hline Ephemeral & Medium & High & High & Medium & Low & Medium & 100 & 0 & 0 \\
\hline Ephemeral & Medium & High & High & Medium & Low & High & 100 & 0 & 0 \\
\hline Ephemeral & Medium & High & High & Medium & Medium & Low & 100 & 0 & 0 \\
\hline Ephemeral & Medium & High & High & Medium & Medium & Medium & 100 & 0 & 0 \\
\hline Ephemeral & Medium & High & High & Medium & Medium & High & 100 & 0 & 0 \\
\hline
\end{tabular}




\begin{tabular}{|c|c|c|c|c|c|c|c|c|c|}
\hline \multicolumn{7}{|c|}{ Inputs } & \multicolumn{3}{|c|}{ Habitat quality } \\
\hline $\begin{array}{c}\text { Water } \\
\text { availability }\end{array}$ & Emergent & Floating & Terrestrial & $\begin{array}{l}\text { Winter } \\
\text { refuge }\end{array}$ & Submerged & $\begin{array}{l}\text { Water } \\
\text { quality }\end{array}$ & Low & Average & High \\
\hline Ephemeral & Medium & High & High & Medium & High & Low & 100 & 0 & 0 \\
\hline Ephemeral & Medium & High & High & Medium & High & Medium & 100 & 0 & 0 \\
\hline Ephemeral & Medium & High & High & Medium & High & High & 100 & 0 & 0 \\
\hline Ephemeral & Medium & High & High & High & Low & Low & 100 & 0 & 0 \\
\hline Ephemeral & Medium & High & High & High & Low & Medium & 100 & 0 & 0 \\
\hline Ephemeral & Medium & High & High & High & Low & High & 100 & 0 & 0 \\
\hline Ephemeral & Medium & High & High & High & Medium & Low & 100 & 0 & 0 \\
\hline Ephemeral & Medium & High & High & High & Medium & Medium & 100 & 0 & 0 \\
\hline Ephemeral & Medium & High & High & High & Medium & High & 100 & 0 & 0 \\
\hline Ephemeral & Medium & High & High & High & High & Low & 100 & 0 & 0 \\
\hline Ephemeral & Medium & High & High & High & High & Medium & 100 & 0 & 0 \\
\hline Ephemeral & Medium & High & High & High & High & High & 100 & 0 & 0 \\
\hline Ephemeral & High & Low & Low & Low & Low & Low & 100 & 0 & 0 \\
\hline Ephemeral & High & Low & Low & Low & Low & Medium & 100 & 0 & 0 \\
\hline Ephemeral & High & Low & Low & Low & Low & High & 100 & 0 & 0 \\
\hline Ephemeral & High & Low & Low & Low & Medium & Low & 100 & 0 & 0 \\
\hline Ephemeral & High & Low & Low & Low & Medium & Medium & 100 & 0 & 0 \\
\hline Ephemeral & High & Low & Low & Low & Medium & High & 100 & 0 & 0 \\
\hline Ephemeral & High & Low & Low & Low & High & Low & 100 & 0 & 0 \\
\hline Ephemeral & High & Low & Low & Low & High & Medium & 100 & 0 & 0 \\
\hline Ephemeral & High & Low & Low & Low & High & High & 100 & 0 & 0 \\
\hline Ephemeral & High & Low & Low & Medium & Low & Low & 100 & 0 & 0 \\
\hline Ephemeral & High & Low & Low & Medium & Low & Medium & 100 & 0 & 0 \\
\hline Ephemeral & High & Low & Low & Medium & Low & High & 100 & 0 & 0 \\
\hline Ephemeral & High & Low & Low & Medium & Medium & Low & 100 & 0 & 0 \\
\hline Ephemeral & High & Low & Low & Medium & Medium & Medium & 100 & 0 & 0 \\
\hline Ephemeral & High & Low & Low & Medium & Medium & High & 100 & 0 & 0 \\
\hline Ephemeral & High & Low & Low & Medium & High & Low & 100 & 0 & 0 \\
\hline Ephemeral & High & Low & Low & Medium & High & Medium & 100 & 0 & 0 \\
\hline Ephemeral & High & Low & Low & Medium & High & High & 100 & 0 & 0 \\
\hline Ephemeral & High & Low & Low & High & Low & Low & 100 & 0 & 0 \\
\hline Ephemeral & High & Low & Low & High & Low & Medium & 100 & 0 & 0 \\
\hline Ephemeral & High & Low & Low & High & Low & High & 100 & 0 & 0 \\
\hline Ephemeral & High & Low & Low & High & Medium & Low & 100 & 0 & 0 \\
\hline Ephemeral & High & Low & Low & High & Medium & Medium & 100 & 0 & 0 \\
\hline Ephemeral & High & Low & Low & High & Medium & High & 100 & 0 & 0 \\
\hline Ephemeral & High & Low & Low & High & High & Low & 100 & 0 & 0 \\
\hline Ephemeral & High & Low & Low & High & High & Medium & 100 & 0 & 0 \\
\hline Ephemeral & High & Low & Low & High & High & High & 100 & 0 & 0 \\
\hline Ephemeral & High & Low & Medium & Low & Low & Low & 100 & 0 & 0 \\
\hline
\end{tabular}




\begin{tabular}{|c|c|c|c|c|c|c|c|c|c|}
\hline \multicolumn{7}{|c|}{ Inputs } & \multicolumn{3}{|c|}{ Habitat quality } \\
\hline $\begin{array}{c}\text { Water } \\
\text { availability }\end{array}$ & Emergent & Floating & Terrestrial & $\begin{array}{l}\text { Winter } \\
\text { refuge }\end{array}$ & Submerged & $\begin{array}{l}\text { Water } \\
\text { quality }\end{array}$ & Low & Average & High \\
\hline Ephemeral & High & Low & Medium & Low & Low & Medium & 100 & 0 & 0 \\
\hline Ephemeral & High & Low & Medium & Low & Low & High & 100 & 0 & 0 \\
\hline Ephemeral & High & Low & Medium & Low & Medium & Low & 100 & 0 & 0 \\
\hline Ephemeral & High & Low & Medium & Low & Medium & Medium & 100 & 0 & 0 \\
\hline Ephemeral & High & Low & Medium & Low & Medium & High & 100 & 0 & 0 \\
\hline Ephemeral & High & Low & Medium & Low & High & Low & 100 & 0 & 0 \\
\hline Ephemeral & High & Low & Medium & Low & High & Medium & 100 & 0 & 0 \\
\hline Ephemeral & High & Low & Medium & Low & High & High & 100 & 0 & 0 \\
\hline Ephemeral & High & Low & Medium & Medium & Low & Low & 100 & 0 & 0 \\
\hline Ephemeral & High & Low & Medium & Medium & Low & Medium & 100 & 0 & 0 \\
\hline Ephemeral & High & Low & Medium & Medium & Low & High & 100 & 0 & 0 \\
\hline Ephemeral & High & Low & Medium & Medium & Medium & Low & 100 & 0 & 0 \\
\hline Ephemeral & High & Low & Medium & Medium & Medium & Medium & 100 & 0 & 0 \\
\hline Ephemeral & High & Low & Medium & Medium & Medium & High & 100 & 0 & 0 \\
\hline Ephemeral & High & Low & Medium & Medium & High & Low & 100 & 0 & 0 \\
\hline Ephemeral & High & Low & Medium & Medium & High & Medium & 100 & 0 & 0 \\
\hline Ephemeral & High & Low & Medium & Medium & High & High & 100 & 0 & 0 \\
\hline Ephemeral & High & Low & Medium & High & Low & Low & 100 & 0 & 0 \\
\hline Ephemeral & High & Low & Medium & High & Low & Medium & 100 & 0 & 0 \\
\hline Ephemeral & High & Low & Medium & High & Low & High & 100 & 0 & 0 \\
\hline Ephemeral & High & Low & Medium & High & Medium & Low & 100 & 0 & 0 \\
\hline Ephemeral & High & Low & Medium & High & Medium & Medium & 100 & 0 & 0 \\
\hline Ephemeral & High & Low & Medium & High & Medium & High & 100 & 0 & 0 \\
\hline Ephemeral & High & Low & Medium & High & High & Low & 100 & 0 & 0 \\
\hline Ephemeral & High & Low & Medium & High & High & Medium & 100 & 0 & 0 \\
\hline Ephemeral & High & Low & Medium & High & High & High & 100 & 0 & 0 \\
\hline Ephemeral & High & Low & High & Low & Low & Low & 100 & 0 & 0 \\
\hline Ephemeral & High & Low & High & Low & Low & Medium & 100 & 0 & 0 \\
\hline Ephemeral & High & Low & High & Low & Low & High & 100 & 0 & 0 \\
\hline Ephemeral & High & Low & High & Low & Medium & Low & 100 & 0 & 0 \\
\hline Ephemeral & High & Low & High & Low & Medium & Medium & 100 & 0 & 0 \\
\hline Ephemeral & High & Low & High & Low & Medium & High & 100 & 0 & 0 \\
\hline Ephemeral & High & Low & High & Low & High & Low & 100 & 0 & 0 \\
\hline Ephemeral & High & Low & High & Low & High & Medium & 100 & 0 & 0 \\
\hline Ephemeral & High & Low & High & Low & High & High & 100 & 0 & 0 \\
\hline Ephemeral & High & Low & High & Medium & Low & Low & 100 & 0 & 0 \\
\hline Ephemeral & High & Low & High & Medium & Low & Medium & 100 & 0 & 0 \\
\hline Ephemeral & High & Low & High & Medium & Low & High & 100 & 0 & 0 \\
\hline Ephemeral & High & Low & High & Medium & Medium & Low & 100 & 0 & 0 \\
\hline Ephemeral & High & Low & High & Medium & Medium & Medium & 100 & 0 & 0 \\
\hline
\end{tabular}




\begin{tabular}{|c|c|c|c|c|c|c|c|c|c|}
\hline \multicolumn{7}{|c|}{ Inputs } & \multicolumn{3}{|c|}{ Habitat quality } \\
\hline $\begin{array}{c}\text { Water } \\
\text { availability }\end{array}$ & Emergent & Floating & Terrestrial & $\begin{array}{l}\text { Winter } \\
\text { refuge }\end{array}$ & Submerged & $\begin{array}{l}\text { Water } \\
\text { quality }\end{array}$ & Low & Average & High \\
\hline Ephemeral & High & Low & High & Medium & Medium & High & 100 & 0 & 0 \\
\hline Ephemeral & High & Low & High & Medium & High & Low & 100 & 0 & 0 \\
\hline Ephemeral & High & Low & High & Medium & High & Medium & 100 & 0 & 0 \\
\hline Ephemeral & High & Low & High & Medium & High & High & 100 & 0 & 0 \\
\hline Ephemeral & High & Low & High & High & Low & Low & 100 & 0 & 0 \\
\hline Ephemeral & High & Low & High & High & Low & Medium & 100 & 0 & 0 \\
\hline Ephemeral & High & Low & High & High & Low & High & 100 & 0 & 0 \\
\hline Ephemeral & High & Low & High & High & Medium & Low & 100 & 0 & 0 \\
\hline Ephemeral & High & Low & High & High & Medium & Medium & 100 & 0 & 0 \\
\hline Ephemeral & High & Low & High & High & Medium & High & 100 & 0 & 0 \\
\hline Ephemeral & High & Low & High & High & High & Low & 100 & 0 & 0 \\
\hline Ephemeral & High & Low & High & High & High & Medium & 100 & 0 & 0 \\
\hline Ephemeral & High & Low & High & High & High & High & 100 & 0 & 0 \\
\hline Ephemeral & High & Medium & Low & Low & Low & Low & 100 & 0 & 0 \\
\hline Ephemeral & High & Medium & Low & Low & Low & Medium & 100 & 0 & 0 \\
\hline Ephemeral & High & Medium & Low & Low & Low & High & 100 & 0 & 0 \\
\hline Ephemeral & High & Medium & Low & Low & Medium & Low & 100 & 0 & 0 \\
\hline Ephemeral & High & Medium & Low & Low & Medium & Medium & 100 & 0 & 0 \\
\hline Ephemeral & High & Medium & Low & Low & Medium & High & 100 & 0 & 0 \\
\hline Ephemeral & High & Medium & Low & Low & High & Low & 100 & 0 & 0 \\
\hline Ephemeral & High & Medium & Low & Low & High & Medium & 100 & 0 & 0 \\
\hline Ephemeral & High & Medium & Low & Low & High & High & 100 & 0 & 0 \\
\hline Ephemeral & High & Medium & Low & Medium & Low & Low & 100 & 0 & 0 \\
\hline Ephemeral & High & Medium & Low & Medium & Low & Medium & 100 & 0 & 0 \\
\hline Ephemeral & High & Medium & Low & Medium & Low & High & 100 & 0 & 0 \\
\hline Ephemeral & High & Medium & Low & Medium & Medium & Low & 100 & 0 & 0 \\
\hline Ephemeral & High & Medium & Low & Medium & Medium & Medium & 100 & 0 & 0 \\
\hline Ephemeral & High & Medium & Low & Medium & Medium & High & 100 & 0 & 0 \\
\hline Ephemeral & High & Medium & Low & Medium & High & Low & 100 & 0 & 0 \\
\hline Ephemeral & High & Medium & Low & Medium & High & Medium & 100 & 0 & 0 \\
\hline Ephemeral & High & Medium & Low & Medium & High & High & 100 & 0 & 0 \\
\hline Ephemeral & High & Medium & Low & High & Low & Low & 100 & 0 & 0 \\
\hline Ephemeral & High & Medium & Low & High & Low & Medium & 100 & 0 & 0 \\
\hline Ephemeral & High & Medium & Low & High & Low & High & 100 & 0 & 0 \\
\hline Ephemeral & High & Medium & Low & High & Medium & Low & 100 & 0 & 0 \\
\hline Ephemeral & High & Medium & Low & High & Medium & Medium & 100 & 0 & 0 \\
\hline Ephemeral & High & Medium & Low & High & Medium & High & 100 & 0 & 0 \\
\hline Ephemeral & High & Medium & Low & High & High & Low & 100 & 0 & 0 \\
\hline Ephemeral & High & Medium & Low & High & High & Medium & 100 & 0 & 0 \\
\hline Ephemeral & High & Medium & Low & High & High & High & 100 & 0 & 0 \\
\hline
\end{tabular}




\begin{tabular}{|c|c|c|c|c|c|c|c|c|c|}
\hline \multicolumn{7}{|c|}{ Inputs } & \multicolumn{3}{|c|}{ Habitat quality } \\
\hline $\begin{array}{c}\text { Water } \\
\text { availability }\end{array}$ & Emergent & Floating & Terrestrial & $\begin{array}{l}\text { Winter } \\
\text { refuge }\end{array}$ & Submerged & $\begin{array}{l}\text { Water } \\
\text { quality }\end{array}$ & Low & Average & High \\
\hline Ephemeral & High & Medium & Medium & Low & Low & Low & 100 & 0 & 0 \\
\hline Ephemeral & High & Medium & Medium & Low & Low & Medium & 100 & 0 & 0 \\
\hline Ephemeral & High & Medium & Medium & Low & Low & High & 100 & 0 & 0 \\
\hline Ephemeral & High & Medium & Medium & Low & Medium & Low & 100 & 0 & 0 \\
\hline Ephemeral & High & Medium & Medium & Low & Medium & Medium & 100 & 0 & 0 \\
\hline Ephemeral & High & Medium & Medium & Low & Medium & High & 100 & 0 & 0 \\
\hline Ephemeral & High & Medium & Medium & Low & High & Low & 100 & 0 & 0 \\
\hline Ephemeral & High & Medium & Medium & Low & High & Medium & 100 & 0 & 0 \\
\hline Ephemeral & High & Medium & Medium & Low & High & High & 100 & 0 & 0 \\
\hline Ephemeral & High & Medium & Medium & Medium & Low & Low & 100 & 0 & 0 \\
\hline Ephemeral & High & Medium & Medium & Medium & Low & Medium & 100 & 0 & 0 \\
\hline Ephemeral & High & Medium & Medium & Medium & Low & High & 100 & 0 & 0 \\
\hline Ephemeral & High & Medium & Medium & Medium & Medium & Low & 100 & 0 & 0 \\
\hline Ephemeral & High & Medium & Medium & Medium & Medium & Medium & 100 & 0 & 0 \\
\hline Ephemeral & High & Medium & Medium & Medium & Medium & High & 100 & 0 & 0 \\
\hline Ephemeral & High & Medium & Medium & Medium & High & Low & 100 & 0 & 0 \\
\hline Ephemeral & High & Medium & Medium & Medium & High & Medium & 100 & 0 & 0 \\
\hline Ephemeral & High & Medium & Medium & Medium & High & High & 100 & 0 & 0 \\
\hline Ephemeral & High & Medium & Medium & High & Low & Low & 100 & 0 & 0 \\
\hline Ephemeral & High & Medium & Medium & High & Low & Medium & 100 & 0 & 0 \\
\hline Ephemeral & High & Medium & Medium & High & Low & High & 100 & 0 & 0 \\
\hline Ephemeral & High & Medium & Medium & High & Medium & Low & 100 & 0 & 0 \\
\hline Ephemeral & High & Medium & Medium & High & Medium & Medium & 100 & 0 & 0 \\
\hline Ephemeral & High & Medium & Medium & High & Medium & High & 100 & 0 & 0 \\
\hline Ephemeral & High & Medium & Medium & High & High & Low & 100 & 0 & 0 \\
\hline Ephemeral & High & Medium & Medium & High & High & Medium & 100 & 0 & 0 \\
\hline Ephemeral & High & Medium & Medium & High & High & High & 100 & 0 & 0 \\
\hline Ephemeral & High & Medium & High & Low & Low & Low & 100 & 0 & 0 \\
\hline Ephemeral & High & Medium & High & Low & Low & Medium & 100 & 0 & 0 \\
\hline Ephemeral & High & Medium & High & Low & Low & High & 100 & 0 & 0 \\
\hline Ephemeral & High & Medium & High & Low & Medium & Low & 100 & 0 & 0 \\
\hline Ephemeral & High & Medium & High & Low & Medium & Medium & 100 & 0 & 0 \\
\hline Ephemeral & High & Medium & High & Low & Medium & High & 100 & 0 & 0 \\
\hline Ephemeral & High & Medium & High & Low & High & Low & 100 & 0 & 0 \\
\hline Ephemeral & High & Medium & High & Low & High & Medium & 100 & 0 & 0 \\
\hline Ephemeral & High & Medium & High & Low & High & High & 100 & 0 & 0 \\
\hline Ephemeral & High & Medium & High & Medium & Low & Low & 100 & 0 & 0 \\
\hline Ephemeral & High & Medium & High & Medium & Low & Medium & 100 & 0 & 0 \\
\hline Ephemeral & High & Medium & High & Medium & Low & High & 100 & 0 & 0 \\
\hline Ephemeral & High & Medium & High & Medium & Medium & Low & 100 & 0 & 0 \\
\hline
\end{tabular}




\begin{tabular}{|c|c|c|c|c|c|c|c|c|c|}
\hline \multicolumn{7}{|c|}{ Inputs } & \multicolumn{3}{|c|}{ Habitat quality } \\
\hline $\begin{array}{c}\text { Water } \\
\text { availability }\end{array}$ & Emergent & Floating & Terrestrial & $\begin{array}{l}\text { Winter } \\
\text { refuge }\end{array}$ & Submerged & $\begin{array}{l}\text { Water } \\
\text { quality }\end{array}$ & Low & Average & High \\
\hline Ephemeral & High & Medium & High & Medium & Medium & Medium & 100 & 0 & 0 \\
\hline Ephemeral & High & Medium & High & Medium & Medium & High & 100 & 0 & 0 \\
\hline Ephemeral & High & Medium & High & Medium & High & Low & 100 & 0 & 0 \\
\hline Ephemeral & High & Medium & High & Medium & High & Medium & 100 & 0 & 0 \\
\hline Ephemeral & High & Medium & High & Medium & High & High & 100 & 0 & 0 \\
\hline Ephemeral & High & Medium & High & High & Low & Low & 100 & 0 & 0 \\
\hline Ephemeral & High & Medium & High & High & Low & Medium & 100 & 0 & 0 \\
\hline Ephemeral & High & Medium & High & High & Low & High & 100 & 0 & 0 \\
\hline Ephemeral & High & Medium & High & High & Medium & Low & 100 & 0 & 0 \\
\hline Ephemeral & High & Medium & High & High & Medium & Medium & 100 & 0 & 0 \\
\hline Ephemeral & High & Medium & High & High & Medium & High & 100 & 0 & 0 \\
\hline Ephemeral & High & Medium & High & High & High & Low & 100 & 0 & 0 \\
\hline Ephemeral & High & Medium & High & High & High & Medium & 100 & 0 & 0 \\
\hline Ephemeral & High & Medium & High & High & High & High & 100 & 0 & 0 \\
\hline Ephemeral & High & High & Low & Low & Low & Low & 100 & 0 & 0 \\
\hline Ephemeral & High & High & Low & Low & Low & Medium & 100 & 0 & 0 \\
\hline Ephemeral & High & High & Low & Low & Low & High & 100 & 0 & 0 \\
\hline Ephemeral & High & High & Low & Low & Medium & Low & 100 & 0 & 0 \\
\hline Ephemeral & High & High & Low & Low & Medium & Medium & 100 & 0 & 0 \\
\hline Ephemeral & High & High & Low & Low & Medium & High & 100 & 0 & 0 \\
\hline Ephemeral & High & High & Low & Low & High & Low & 100 & 0 & 0 \\
\hline Ephemeral & High & High & Low & Low & High & Medium & 100 & 0 & 0 \\
\hline Ephemeral & High & High & Low & Low & High & High & 100 & 0 & 0 \\
\hline Ephemeral & High & High & Low & Medium & Low & Low & 100 & 0 & 0 \\
\hline Ephemeral & High & High & Low & Medium & Low & Medium & 100 & 0 & 0 \\
\hline Ephemeral & High & High & Low & Medium & Low & High & 100 & 0 & 0 \\
\hline Ephemeral & High & High & Low & Medium & Medium & Low & 100 & 0 & 0 \\
\hline Ephemeral & High & High & Low & Medium & Medium & Medium & 100 & 0 & 0 \\
\hline Ephemeral & High & High & Low & Medium & Medium & High & 100 & 0 & 0 \\
\hline Ephemeral & High & High & Low & Medium & High & Low & 100 & 0 & 0 \\
\hline Ephemeral & High & High & Low & Medium & High & Medium & 100 & 0 & 0 \\
\hline Ephemeral & High & High & Low & Medium & High & High & 100 & 0 & 0 \\
\hline Ephemeral & High & High & Low & High & Low & Low & 100 & 0 & 0 \\
\hline Ephemeral & High & High & Low & High & Low & Medium & 100 & 0 & 0 \\
\hline Ephemeral & High & High & Low & High & Low & High & 100 & 0 & 0 \\
\hline Ephemeral & High & High & Low & High & Medium & Low & 100 & 0 & 0 \\
\hline Ephemeral & High & High & Low & High & Medium & Medium & 100 & 0 & 0 \\
\hline Ephemeral & High & High & Low & High & Medium & High & 100 & 0 & 0 \\
\hline Ephemeral & High & High & Low & High & High & Low & 100 & 0 & 0 \\
\hline Ephemeral & High & High & Low & High & High & Medium & 100 & 0 & 0 \\
\hline
\end{tabular}




\begin{tabular}{|c|c|c|c|c|c|c|c|c|c|}
\hline \multicolumn{7}{|c|}{ Inputs } & \multicolumn{3}{|c|}{ Habitat quality } \\
\hline $\begin{array}{c}\text { Water } \\
\text { availability }\end{array}$ & Emergent & Floating & Terrestrial & $\begin{array}{l}\text { Winter } \\
\text { refuge }\end{array}$ & Submerged & $\begin{array}{l}\text { Water } \\
\text { quality }\end{array}$ & Low & Average & High \\
\hline Ephemeral & High & High & Low & High & High & High & 100 & 0 & 0 \\
\hline Ephemeral & High & High & Medium & Low & Low & Low & 100 & 0 & 0 \\
\hline Ephemeral & High & High & Medium & Low & Low & Medium & 100 & 0 & 0 \\
\hline Ephemeral & High & High & Medium & Low & Low & High & 100 & 0 & 0 \\
\hline Ephemeral & High & High & Medium & Low & Medium & Low & 100 & 0 & 0 \\
\hline Ephemeral & High & High & Medium & Low & Medium & Medium & 100 & 0 & 0 \\
\hline Ephemeral & High & High & Medium & Low & Medium & High & 100 & 0 & 0 \\
\hline Ephemeral & High & High & Medium & Low & High & Low & 100 & 0 & 0 \\
\hline Ephemeral & High & High & Medium & Low & High & Medium & 100 & 0 & 0 \\
\hline Ephemeral & High & High & Medium & Low & High & High & 100 & 0 & 0 \\
\hline Ephemeral & High & High & Medium & Medium & Low & Low & 100 & 0 & 0 \\
\hline Ephemeral & High & High & Medium & Medium & Low & Medium & 100 & 0 & 0 \\
\hline Ephemeral & High & High & Medium & Medium & Low & High & 100 & 0 & 0 \\
\hline Ephemeral & High & High & Medium & Medium & Medium & Low & 100 & 0 & 0 \\
\hline Ephemeral & High & High & Medium & Medium & Medium & Medium & 100 & 0 & 0 \\
\hline Ephemeral & High & High & Medium & Medium & Medium & High & 100 & 0 & 0 \\
\hline Ephemeral & High & High & Medium & Medium & High & Low & 100 & 0 & 0 \\
\hline Ephemeral & High & High & Medium & Medium & High & Medium & 100 & 0 & 0 \\
\hline Ephemeral & High & High & Medium & Medium & High & High & 100 & 0 & 0 \\
\hline Ephemeral & High & High & Medium & High & Low & Low & 100 & 0 & 0 \\
\hline Ephemeral & High & High & Medium & High & Low & Medium & 100 & 0 & 0 \\
\hline Ephemeral & High & High & Medium & High & Low & High & 100 & 0 & 0 \\
\hline Ephemeral & High & High & Medium & High & Medium & Low & 100 & 0 & 0 \\
\hline Ephemeral & High & High & Medium & High & Medium & Medium & 100 & 0 & 0 \\
\hline Ephemeral & High & High & Medium & High & Medium & High & 100 & 0 & 0 \\
\hline Ephemeral & High & High & Medium & High & High & Low & 100 & 0 & 0 \\
\hline Ephemeral & High & High & Medium & High & High & Medium & 100 & 0 & 0 \\
\hline Ephemeral & High & High & Medium & High & High & High & 100 & 0 & 0 \\
\hline Ephemeral & High & High & High & Low & Low & Low & 100 & 0 & 0 \\
\hline Ephemeral & High & High & High & Low & Low & Medium & 100 & 0 & 0 \\
\hline Ephemeral & High & High & High & Low & Low & High & 100 & 0 & 0 \\
\hline Ephemeral & High & High & High & Low & Medium & Low & 100 & 0 & 0 \\
\hline Ephemeral & High & High & High & Low & Medium & Medium & 100 & 0 & 0 \\
\hline Ephemeral & High & High & High & Low & Medium & High & 100 & 0 & 0 \\
\hline Ephemeral & High & High & High & Low & High & Low & 100 & 0 & 0 \\
\hline Ephemeral & High & High & High & Low & High & Medium & 100 & 0 & 0 \\
\hline Ephemeral & High & High & High & Low & High & High & 100 & 0 & 0 \\
\hline Ephemeral & High & High & High & Medium & Low & Low & 100 & 0 & 0 \\
\hline Ephemeral & High & High & High & Medium & Low & Medium & 100 & 0 & 0 \\
\hline Ephemeral & High & High & High & Medium & Low & High & 100 & 0 & 0 \\
\hline
\end{tabular}




\begin{tabular}{|c|c|c|c|c|c|c|c|c|c|}
\hline \multicolumn{7}{|c|}{ Inputs } & \multicolumn{3}{|c|}{ Habitat quality } \\
\hline $\begin{array}{c}\text { Water } \\
\text { availability }\end{array}$ & Emergent & Floating & Terrestrial & $\begin{array}{l}\text { Winter } \\
\text { refuge }\end{array}$ & Submerged & $\begin{array}{l}\text { Water } \\
\text { quality }\end{array}$ & Low & Average & High \\
\hline Ephemeral & High & High & High & Medium & Medium & Low & 100 & 0 & 0 \\
\hline Ephemeral & High & High & High & Medium & Medium & Medium & 100 & 0 & 0 \\
\hline Ephemeral & High & High & High & Medium & Medium & High & 100 & 0 & 0 \\
\hline Ephemeral & High & High & High & Medium & High & Low & 100 & 0 & 0 \\
\hline Ephemeral & High & High & High & Medium & High & Medium & 100 & 0 & 0 \\
\hline Ephemeral & High & High & High & Medium & High & High & 100 & 0 & 0 \\
\hline Ephemeral & High & High & High & High & Low & Low & 100 & 0 & 0 \\
\hline Ephemeral & High & High & High & High & Low & Medium & 100 & 0 & 0 \\
\hline Ephemeral & High & High & High & High & Low & High & 100 & 0 & 0 \\
\hline Ephemeral & High & High & High & High & Medium & Low & 100 & 0 & 0 \\
\hline Ephemeral & High & High & High & High & Medium & Medium & 100 & 0 & 0 \\
\hline Ephemeral & High & High & High & High & Medium & High & 100 & 0 & 0 \\
\hline Ephemeral & High & High & High & High & High & Low & 100 & 0 & 0 \\
\hline Ephemeral & High & High & High & High & High & Medium & 100 & 0 & 0 \\
\hline Ephemeral & High & High & High & High & High & High & 100 & 0 & 0 \\
\hline Winter & Low & Low & Low & Low & Low & Low & 100 & 0 & 0 \\
\hline Winter & Low & Low & Low & Low & Low & Medium & 100 & 0 & 0 \\
\hline Winter & Low & Low & Low & Low & Low & High & 100 & 0 & 0 \\
\hline Winter & Low & Low & Low & Low & Medium & Low & 100 & 0 & 0 \\
\hline Winter & Low & Low & Low & Low & Medium & Medium & 100 & 0 & 0 \\
\hline Winter & Low & Low & Low & Low & Medium & High & 100 & 0 & 0 \\
\hline Winter & Low & Low & Low & Low & High & Low & 100 & 0 & 0 \\
\hline Winter & Low & Low & Low & Low & High & Medium & 100 & 0 & 0 \\
\hline Winter & Low & Low & Low & Low & High & High & 100 & 0 & 0 \\
\hline Winter & Low & Low & Low & Medium & Low & Low & 100 & 0 & 0 \\
\hline Winter & Low & Low & Low & Medium & Low & Medium & 100 & 0 & 0 \\
\hline Winter & Low & Low & Low & Medium & Low & High & 100 & 0 & 0 \\
\hline Winter & Low & Low & Low & Medium & Medium & Low & 100 & 0 & 0 \\
\hline Winter & Low & Low & Low & Medium & Medium & Medium & 100 & 0 & 0 \\
\hline Winter & Low & Low & Low & Medium & Medium & High & 100 & 0 & 0 \\
\hline Winter & Low & Low & Low & Medium & High & Low & 100 & 0 & 0 \\
\hline Winter & Low & Low & Low & Medium & High & Medium & 100 & 0 & 0 \\
\hline Winter & Low & Low & Low & Medium & High & High & 100 & 0 & 0 \\
\hline Winter & Low & Low & Low & High & Low & Low & 100 & 0 & 0 \\
\hline Winter & Low & Low & Low & High & Low & Medium & 100 & 0 & 0 \\
\hline Winter & Low & Low & Low & High & Low & High & 100 & 0 & 0 \\
\hline Winter & Low & Low & Low & High & Medium & Low & 100 & 0 & 0 \\
\hline Winter & Low & Low & Low & High & Medium & Medium & 100 & 0 & 0 \\
\hline Winter & Low & Low & Low & High & Medium & High & 100 & 0 & 0 \\
\hline Winter & Low & Low & Low & High & High & Low & 100 & 0 & 0 \\
\hline
\end{tabular}




\begin{tabular}{|c|c|c|c|c|c|c|c|c|c|}
\hline \multicolumn{7}{|c|}{ Inputs } & \multicolumn{3}{|c|}{ Habitat quality } \\
\hline $\begin{array}{c}\text { Water } \\
\text { availability }\end{array}$ & Emergent & Floating & Terrestrial & $\begin{array}{l}\text { Winter } \\
\text { refuge }\end{array}$ & Submerged & $\begin{array}{l}\text { Water } \\
\text { quality }\end{array}$ & Low & Average & High \\
\hline Winter & Low & Low & Low & High & High & Medium & 100 & 0 & 0 \\
\hline Winter & Low & Low & Low & High & High & High & 100 & 0 & 0 \\
\hline Winter & Low & Low & Medium & Low & Low & Low & 100 & 0 & 0 \\
\hline Winter & Low & Low & Medium & Low & Low & Medium & 100 & 0 & 0 \\
\hline Winter & Low & Low & Medium & Low & Low & High & 100 & 0 & 0 \\
\hline Winter & Low & Low & Medium & Low & Medium & Low & 100 & 0 & 0 \\
\hline Winter & Low & Low & Medium & Low & Medium & Medium & 100 & 0 & 0 \\
\hline Winter & Low & Low & Medium & Low & Medium & High & 100 & 0 & 0 \\
\hline Winter & Low & Low & Medium & Low & High & Low & 100 & 0 & 0 \\
\hline Winter & Low & Low & Medium & Low & High & Medium & 100 & 0 & 0 \\
\hline Winter & Low & Low & Medium & Low & High & High & 100 & 0 & 0 \\
\hline Winter & Low & Low & Medium & Medium & Low & Low & 100 & 0 & 0 \\
\hline Winter & Low & Low & Medium & Medium & Low & Medium & 100 & 0 & 0 \\
\hline Winter & Low & Low & Medium & Medium & Low & High & 100 & 0 & 0 \\
\hline Winter & Low & Low & Medium & Medium & Medium & Low & 100 & 0 & 0 \\
\hline Winter & Low & Low & Medium & Medium & Medium & Medium & 100 & 0 & 0 \\
\hline Winter & Low & Low & Medium & Medium & Medium & High & 100 & 0 & 0 \\
\hline Winter & Low & Low & Medium & Medium & High & Low & 100 & 0 & 0 \\
\hline Winter & Low & Low & Medium & Medium & High & Medium & 100 & 0 & 0 \\
\hline Winter & Low & Low & Medium & Medium & High & High & 100 & 0 & 0 \\
\hline Winter & Low & Low & Medium & High & Low & Low & 100 & 0 & 0 \\
\hline Winter & Low & Low & Medium & High & Low & Medium & 100 & 0 & 0 \\
\hline Winter & Low & Low & Medium & High & Low & High & 100 & 0 & 0 \\
\hline Winter & Low & Low & Medium & High & Medium & Low & 100 & 0 & 0 \\
\hline Winter & Low & Low & Medium & High & Medium & Medium & 100 & 0 & 0 \\
\hline Winter & Low & Low & Medium & High & Medium & High & 100 & 0 & 0 \\
\hline Winter & Low & Low & Medium & High & High & Low & 100 & 0 & 0 \\
\hline Winter & Low & Low & Medium & High & High & Medium & 100 & 0 & 0 \\
\hline Winter & Low & Low & Medium & High & High & High & 100 & 0 & 0 \\
\hline Winter & Low & Low & High & Low & Low & Low & 100 & 0 & 0 \\
\hline Winter & Low & Low & High & Low & Low & Medium & 100 & 0 & 0 \\
\hline Winter & Low & Low & High & Low & Low & High & 100 & 0 & 0 \\
\hline Winter & Low & Low & High & Low & Medium & Low & 100 & 0 & 0 \\
\hline Winter & Low & Low & High & Low & Medium & Medium & 100 & 0 & 0 \\
\hline Winter & Low & Low & High & Low & Medium & High & 100 & 0 & 0 \\
\hline Winter & Low & Low & High & Low & High & Low & 100 & 0 & 0 \\
\hline Winter & Low & Low & High & Low & High & Medium & 100 & 0 & 0 \\
\hline Winter & Low & Low & High & Low & High & High & 100 & 0 & 0 \\
\hline Winter & Low & Low & High & Medium & Low & Low & 100 & 0 & 0 \\
\hline Winter & Low & Low & High & Medium & Low & Medium & 100 & 0 & 0 \\
\hline
\end{tabular}




\begin{tabular}{|c|c|c|c|c|c|c|c|c|c|}
\hline \multicolumn{7}{|c|}{ Inputs } & \multicolumn{3}{|c|}{ Habitat quality } \\
\hline $\begin{array}{c}\text { Water } \\
\text { availability }\end{array}$ & Emergent & Floating & Terrestrial & $\begin{array}{l}\text { Winter } \\
\text { refuge }\end{array}$ & Submerged & $\begin{array}{l}\text { Water } \\
\text { quality }\end{array}$ & Low & Average & High \\
\hline Winter & Low & Low & High & Medium & Low & High & 100 & 0 & 0 \\
\hline Winter & Low & Low & High & Medium & Medium & Low & 100 & 0 & 0 \\
\hline Winter & Low & Low & High & Medium & Medium & Medium & 100 & 0 & 0 \\
\hline Winter & Low & Low & High & Medium & Medium & High & 100 & 0 & 0 \\
\hline Winter & Low & Low & High & Medium & High & Low & 100 & 0 & 0 \\
\hline Winter & Low & Low & High & Medium & High & Medium & 100 & 0 & 0 \\
\hline Winter & Low & Low & High & Medium & High & High & 100 & 0 & 0 \\
\hline Winter & Low & Low & High & High & Low & Low & 100 & 0 & 0 \\
\hline Winter & Low & Low & High & High & Low & Medium & 100 & 0 & 0 \\
\hline Winter & Low & Low & High & High & Low & High & 100 & 0 & 0 \\
\hline Winter & Low & Low & High & High & Medium & Low & 100 & 0 & 0 \\
\hline Winter & Low & Low & High & High & Medium & Medium & 100 & 0 & 0 \\
\hline Winter & Low & Low & High & High & Medium & High & 100 & 0 & 0 \\
\hline Winter & Low & Low & High & High & High & Low & 100 & 0 & 0 \\
\hline Winter & Low & Low & High & High & High & Medium & 100 & 0 & 0 \\
\hline Winter & Low & Low & High & High & High & High & 100 & 0 & 0 \\
\hline Winter & Low & Medium & Low & Low & Low & Low & 100 & 0 & 0 \\
\hline Winter & Low & Medium & Low & Low & Low & Medium & 100 & 0 & 0 \\
\hline Winter & Low & Medium & Low & Low & Low & High & 100 & 0 & 0 \\
\hline Winter & Low & Medium & Low & Low & Medium & Low & 100 & 0 & 0 \\
\hline Winter & Low & Medium & Low & Low & Medium & Medium & 100 & 0 & 0 \\
\hline Winter & Low & Medium & Low & Low & Medium & High & 100 & 0 & 0 \\
\hline Winter & Low & Medium & Low & Low & High & Low & 100 & 0 & 0 \\
\hline Winter & Low & Medium & Low & Low & High & Medium & 100 & 0 & 0 \\
\hline Winter & Low & Medium & Low & Low & High & High & 100 & 0 & 0 \\
\hline Winter & Low & Medium & Low & Medium & Low & Low & 100 & 0 & 0 \\
\hline Winter & Low & Medium & Low & Medium & Low & Medium & 100 & 0 & 0 \\
\hline Winter & Low & Medium & Low & Medium & Low & High & 100 & 0 & 0 \\
\hline Winter & Low & Medium & Low & Medium & Medium & Low & 100 & 0 & 0 \\
\hline Winter & Low & Medium & Low & Medium & Medium & Medium & 100 & 0 & 0 \\
\hline Winter & Low & Medium & Low & Medium & Medium & High & 100 & 0 & 0 \\
\hline Winter & Low & Medium & Low & Medium & High & Low & 100 & 0 & 0 \\
\hline Winter & Low & Medium & Low & Medium & High & Medium & 100 & 0 & 0 \\
\hline Winter & Low & Medium & Low & Medium & High & High & 100 & 0 & 0 \\
\hline Winter & Low & Medium & Low & High & Low & Low & 100 & 0 & 0 \\
\hline Winter & Low & Medium & Low & High & Low & Medium & 100 & 0 & 0 \\
\hline Winter & Low & Medium & Low & High & Low & High & 100 & 0 & 0 \\
\hline Winter & Low & Medium & Low & High & Medium & Low & 100 & 0 & 0 \\
\hline Winter & Low & Medium & Low & High & Medium & Medium & 100 & 0 & 0 \\
\hline Winter & Low & Medium & Low & High & Medium & High & 100 & 0 & 0 \\
\hline
\end{tabular}




\begin{tabular}{|c|c|c|c|c|c|c|c|c|c|}
\hline \multicolumn{7}{|c|}{ Inputs } & \multicolumn{3}{|c|}{ Habitat quality } \\
\hline $\begin{array}{c}\text { Water } \\
\text { availability }\end{array}$ & Emergent & Floating & Terrestrial & $\begin{array}{l}\text { Winter } \\
\text { refuge }\end{array}$ & Submerged & $\begin{array}{l}\text { Water } \\
\text { quality }\end{array}$ & Low & Average & High \\
\hline Winter & Low & Medium & Low & High & High & Low & 100 & 0 & 0 \\
\hline Winter & Low & Medium & Low & High & High & Medium & 100 & 0 & 0 \\
\hline Winter & Low & Medium & Low & High & High & High & 100 & 0 & 0 \\
\hline Winter & Low & Medium & Medium & Low & Low & Low & 100 & 0 & 0 \\
\hline Winter & Low & Medium & Medium & Low & Low & Medium & 100 & 0 & 0 \\
\hline Winter & Low & Medium & Medium & Low & Low & High & 100 & 0 & 0 \\
\hline Winter & Low & Medium & Medium & Low & Medium & Low & 100 & 0 & 0 \\
\hline Winter & Low & Medium & Medium & Low & Medium & Medium & 100 & 0 & 0 \\
\hline Winter & Low & Medium & Medium & Low & Medium & High & 100 & 0 & 0 \\
\hline Winter & Low & Medium & Medium & Low & High & Low & 100 & 0 & 0 \\
\hline Winter & Low & Medium & Medium & Low & High & Medium & 100 & 0 & 0 \\
\hline Winter & Low & Medium & Medium & Low & High & High & 100 & 0 & 0 \\
\hline Winter & Low & Medium & Medium & Medium & Low & Low & 100 & 0 & 0 \\
\hline Winter & Low & Medium & Medium & Medium & Low & Medium & 100 & 0 & 0 \\
\hline Winter & Low & Medium & Medium & Medium & Low & High & 100 & 0 & 0 \\
\hline Winter & Low & Medium & Medium & Medium & Medium & Low & 100 & 0 & 0 \\
\hline Winter & Low & Medium & Medium & Medium & Medium & Medium & 100 & 0 & 0 \\
\hline Winter & Low & Medium & Medium & Medium & Medium & High & 100 & 0 & 0 \\
\hline Winter & Low & Medium & Medium & Medium & High & Low & 100 & 0 & 0 \\
\hline Winter & Low & Medium & Medium & Medium & High & Medium & 100 & 0 & 0 \\
\hline Winter & Low & Medium & Medium & Medium & High & High & 100 & 0 & 0 \\
\hline Winter & Low & Medium & Medium & High & Low & Low & 100 & 0 & 0 \\
\hline Winter & Low & Medium & Medium & High & Low & Medium & 100 & 0 & 0 \\
\hline Winter & Low & Medium & Medium & High & Low & High & 100 & 0 & 0 \\
\hline Winter & Low & Medium & Medium & High & Medium & Low & 100 & 0 & 0 \\
\hline Winter & Low & Medium & Medium & High & Medium & Medium & 100 & 0 & 0 \\
\hline Winter & Low & Medium & Medium & High & Medium & High & 100 & 0 & 0 \\
\hline Winter & Low & Medium & Medium & High & High & Low & 100 & 0 & 0 \\
\hline Winter & Low & Medium & Medium & High & High & Medium & 100 & 0 & 0 \\
\hline Winter & Low & Medium & Medium & High & High & High & 100 & 0 & 0 \\
\hline Winter & Low & Medium & High & Low & Low & Low & 100 & 0 & 0 \\
\hline Winter & Low & Medium & High & Low & Low & Medium & 100 & 0 & 0 \\
\hline Winter & Low & Medium & High & Low & Low & High & 100 & 0 & 0 \\
\hline Winter & Low & Medium & High & Low & Medium & Low & 100 & 0 & 0 \\
\hline Winter & Low & Medium & High & Low & Medium & Medium & 100 & 0 & 0 \\
\hline Winter & Low & Medium & High & Low & Medium & High & 100 & 0 & 0 \\
\hline Winter & Low & Medium & High & Low & High & Low & 100 & 0 & 0 \\
\hline Winter & Low & Medium & High & Low & High & Medium & 100 & 0 & 0 \\
\hline Winter & Low & Medium & High & Low & High & High & 100 & 0 & 0 \\
\hline Winter & Low & Medium & High & Medium & Low & Low & 100 & 0 & 0 \\
\hline
\end{tabular}




\begin{tabular}{|c|c|c|c|c|c|c|c|c|c|}
\hline \multicolumn{7}{|c|}{ Inputs } & \multicolumn{3}{|c|}{ Habitat quality } \\
\hline $\begin{array}{c}\text { Water } \\
\text { availability }\end{array}$ & Emergent & Floating & Terrestrial & $\begin{array}{l}\text { Winter } \\
\text { refuge }\end{array}$ & Submerged & $\begin{array}{l}\text { Water } \\
\text { quality }\end{array}$ & Low & Average & High \\
\hline Winter & Low & Medium & High & Medium & Low & Medium & 100 & 0 & 0 \\
\hline Winter & Low & Medium & High & Medium & Low & High & 100 & 0 & 0 \\
\hline Winter & Low & Medium & High & Medium & Medium & Low & 100 & 0 & 0 \\
\hline Winter & Low & Medium & High & Medium & Medium & Medium & 100 & 0 & 0 \\
\hline Winter & Low & Medium & High & Medium & Medium & High & 100 & 0 & 0 \\
\hline Winter & Low & Medium & High & Medium & High & Low & 100 & 0 & 0 \\
\hline Winter & Low & Medium & High & Medium & High & Medium & 100 & 0 & 0 \\
\hline Winter & Low & Medium & High & Medium & High & High & 100 & 0 & 0 \\
\hline Winter & Low & Medium & High & High & Low & Low & 100 & 0 & 0 \\
\hline Winter & Low & Medium & High & High & Low & Medium & 100 & 0 & 0 \\
\hline Winter & Low & Medium & High & High & Low & High & 100 & 0 & 0 \\
\hline Winter & Low & Medium & High & High & Medium & Low & 100 & 0 & 0 \\
\hline Winter & Low & Medium & High & High & Medium & Medium & 100 & 0 & 0 \\
\hline Winter & Low & Medium & High & High & Medium & High & 100 & 0 & 0 \\
\hline Winter & Low & Medium & High & High & High & Low & 100 & 0 & 0 \\
\hline Winter & Low & Medium & High & High & High & Medium & 100 & 0 & 0 \\
\hline Winter & Low & Medium & High & High & High & High & 100 & 0 & 0 \\
\hline Winter & Low & High & Low & Low & Low & Low & 100 & 0 & 0 \\
\hline Winter & Low & High & Low & Low & Low & Medium & 100 & 0 & 0 \\
\hline Winter & Low & High & Low & Low & Low & High & 100 & 0 & 0 \\
\hline Winter & Low & High & Low & Low & Medium & Low & 100 & 0 & 0 \\
\hline Winter & Low & High & Low & Low & Medium & Medium & 100 & 0 & 0 \\
\hline Winter & Low & High & Low & Low & Medium & High & 100 & 0 & 0 \\
\hline Winter & Low & High & Low & Low & High & Low & 100 & 0 & 0 \\
\hline Winter & Low & High & Low & Low & High & Medium & 100 & 0 & 0 \\
\hline Winter & Low & High & Low & Low & High & High & 100 & 0 & 0 \\
\hline Winter & Low & High & Low & Medium & Low & Low & 100 & 0 & 0 \\
\hline Winter & Low & High & Low & Medium & Low & Medium & 100 & 0 & 0 \\
\hline Winter & Low & High & Low & Medium & Low & High & 100 & 0 & 0 \\
\hline Winter & Low & High & Low & Medium & Medium & Low & 100 & 0 & 0 \\
\hline Winter & Low & High & Low & Medium & Medium & Medium & 100 & 0 & 0 \\
\hline Winter & Low & High & Low & Medium & Medium & High & 100 & 0 & 0 \\
\hline Winter & Low & High & Low & Medium & High & Low & 100 & 0 & 0 \\
\hline Winter & Low & High & Low & Medium & High & Medium & 100 & 0 & 0 \\
\hline Winter & Low & High & Low & Medium & High & High & 100 & 0 & 0 \\
\hline Winter & Low & High & Low & High & Low & Low & 100 & 0 & 0 \\
\hline Winter & Low & High & Low & High & Low & Medium & 100 & 0 & 0 \\
\hline Winter & Low & High & Low & High & Low & High & 100 & 0 & 0 \\
\hline Winter & Low & High & Low & High & Medium & Low & 100 & 0 & 0 \\
\hline Winter & Low & High & Low & High & Medium & Medium & 100 & 0 & 0 \\
\hline
\end{tabular}




\begin{tabular}{|c|c|c|c|c|c|c|c|c|c|}
\hline \multicolumn{7}{|c|}{ Inputs } & \multicolumn{3}{|c|}{ Habitat quality } \\
\hline $\begin{array}{c}\text { Water } \\
\text { availability }\end{array}$ & Emergent & Floating & Terrestrial & $\begin{array}{l}\text { Winter } \\
\text { refuge }\end{array}$ & Submerged & $\begin{array}{l}\text { Water } \\
\text { quality }\end{array}$ & Low & Average & High \\
\hline Winter & Low & High & Low & High & Medium & High & 100 & 0 & 0 \\
\hline Winter & Low & High & Low & High & High & Low & 100 & 0 & 0 \\
\hline Winter & Low & High & Low & High & High & Medium & 100 & 0 & 0 \\
\hline Winter & Low & High & Low & High & High & High & 100 & 0 & 0 \\
\hline Winter & Low & High & Medium & Low & Low & Low & 100 & 0 & 0 \\
\hline Winter & Low & High & Medium & Low & Low & Medium & 100 & 0 & 0 \\
\hline Winter & Low & High & Medium & Low & Low & High & 100 & 0 & 0 \\
\hline Winter & Low & High & Medium & Low & Medium & Low & 100 & 0 & 0 \\
\hline Winter & Low & High & Medium & Low & Medium & Medium & 100 & 0 & 0 \\
\hline Winter & Low & High & Medium & Low & Medium & High & 100 & 0 & 0 \\
\hline Winter & Low & High & Medium & Low & High & Low & 100 & 0 & 0 \\
\hline Winter & Low & High & Medium & Low & High & Medium & 100 & 0 & 0 \\
\hline Winter & Low & High & Medium & Low & High & High & 100 & 0 & 0 \\
\hline Winter & Low & High & Medium & Medium & Low & Low & 100 & 0 & 0 \\
\hline Winter & Low & High & Medium & Medium & Low & Medium & 100 & 0 & 0 \\
\hline Winter & Low & High & Medium & Medium & Low & High & 100 & 0 & 0 \\
\hline Winter & Low & High & Medium & Medium & Medium & Low & 100 & 0 & 0 \\
\hline Winter & Low & High & Medium & Medium & Medium & Medium & 100 & 0 & 0 \\
\hline Winter & Low & High & Medium & Medium & Medium & High & 100 & 0 & 0 \\
\hline Winter & Low & High & Medium & Medium & High & Low & 100 & 0 & 0 \\
\hline Winter & Low & High & Medium & Medium & High & Medium & 100 & 0 & 0 \\
\hline Winter & Low & High & Medium & Medium & High & High & 100 & 0 & 0 \\
\hline Winter & Low & High & Medium & High & Low & Low & 100 & 0 & 0 \\
\hline Winter & Low & High & Medium & High & Low & Medium & 100 & 0 & 0 \\
\hline Winter & Low & High & Medium & High & Low & High & 100 & 0 & 0 \\
\hline Winter & Low & High & Medium & High & Medium & Low & 100 & 0 & 0 \\
\hline Winter & Low & High & Medium & High & Medium & Medium & 100 & 0 & 0 \\
\hline Winter & Low & High & Medium & High & Medium & High & 100 & 0 & 0 \\
\hline Winter & Low & High & Medium & High & High & Low & 100 & 0 & 0 \\
\hline Winter & Low & High & Medium & High & High & Medium & 100 & 0 & 0 \\
\hline Winter & Low & High & Medium & High & High & High & 100 & 0 & 0 \\
\hline Winter & Low & High & High & Low & Low & Low & 100 & 0 & 0 \\
\hline Winter & Low & High & High & Low & Low & Medium & 100 & 0 & 0 \\
\hline Winter & Low & High & High & Low & Low & High & 100 & 0 & 0 \\
\hline Winter & Low & High & High & Low & Medium & Low & 100 & 0 & 0 \\
\hline Winter & Low & High & High & Low & Medium & Medium & 100 & 0 & 0 \\
\hline Winter & Low & High & High & Low & Medium & High & 100 & 0 & 0 \\
\hline Winter & Low & High & High & Low & High & Low & 100 & 0 & 0 \\
\hline Winter & Low & High & High & Low & High & Medium & 100 & 0 & 0 \\
\hline Winter & Low & High & High & Low & High & High & 100 & 0 & 0 \\
\hline
\end{tabular}




\begin{tabular}{|c|c|c|c|c|c|c|c|c|c|}
\hline \multicolumn{7}{|c|}{ Inputs } & \multicolumn{3}{|c|}{ Habitat quality } \\
\hline $\begin{array}{c}\text { Water } \\
\text { availability }\end{array}$ & Emergent & Floating & Terrestrial & $\begin{array}{l}\text { Winter } \\
\text { refuge }\end{array}$ & Submerged & $\begin{array}{l}\text { Water } \\
\text { quality }\end{array}$ & Low & Average & High \\
\hline Winter & Low & High & High & Medium & Low & Low & 100 & 0 & 0 \\
\hline Winter & Low & High & High & Medium & Low & Medium & 100 & 0 & 0 \\
\hline Winter & Low & High & High & Medium & Low & High & 100 & 0 & 0 \\
\hline Winter & Low & High & High & Medium & Medium & Low & 100 & 0 & 0 \\
\hline Winter & Low & High & High & Medium & Medium & Medium & 100 & 0 & 0 \\
\hline Winter & Low & High & High & Medium & Medium & High & 100 & 0 & 0 \\
\hline Winter & Low & High & High & Medium & High & Low & 100 & 0 & 0 \\
\hline Winter & Low & High & High & Medium & High & Medium & 100 & 0 & 0 \\
\hline Winter & Low & High & High & Medium & High & High & 100 & 0 & 0 \\
\hline Winter & Low & High & High & High & Low & Low & 100 & 0 & 0 \\
\hline Winter & Low & High & High & High & Low & Medium & 100 & 0 & 0 \\
\hline Winter & Low & High & High & High & Low & High & 100 & 0 & 0 \\
\hline Winter & Low & High & High & High & Medium & Low & 100 & 0 & 0 \\
\hline Winter & Low & High & High & High & Medium & Medium & 100 & 0 & 0 \\
\hline Winter & Low & High & High & High & Medium & High & 100 & 0 & 0 \\
\hline Winter & Low & High & High & High & High & Low & 100 & 0 & 0 \\
\hline Winter & Low & High & High & High & High & Medium & 100 & 0 & 0 \\
\hline Winter & Low & High & High & High & High & High & 100 & 0 & 0 \\
\hline Winter & Medium & Low & Low & Low & Low & Low & 100 & 0 & 0 \\
\hline Winter & Medium & Low & Low & Low & Low & Medium & 100 & 0 & 0 \\
\hline Winter & Medium & Low & Low & Low & Low & High & 100 & 0 & 0 \\
\hline Winter & Medium & Low & Low & Low & Medium & Low & 100 & 0 & 0 \\
\hline Winter & Medium & Low & Low & Low & Medium & Medium & 100 & 0 & 0 \\
\hline Winter & Medium & Low & Low & Low & Medium & High & 100 & 0 & 0 \\
\hline Winter & Medium & Low & Low & Low & High & Low & 100 & 0 & 0 \\
\hline Winter & Medium & Low & Low & Low & High & Medium & 100 & 0 & 0 \\
\hline Winter & Medium & Low & Low & Low & High & High & 100 & 0 & 0 \\
\hline Winter & Medium & Low & Low & Medium & Low & Low & 100 & 0 & 0 \\
\hline Winter & Medium & Low & Low & Medium & Low & Medium & 100 & 0 & 0 \\
\hline Winter & Medium & Low & Low & Medium & Low & High & 100 & 0 & 0 \\
\hline Winter & Medium & Low & Low & Medium & Medium & Low & 100 & 0 & 0 \\
\hline Winter & Medium & Low & Low & Medium & Medium & Medium & 100 & 0 & 0 \\
\hline Winter & Medium & Low & Low & Medium & Medium & High & 100 & 0 & 0 \\
\hline Winter & Medium & Low & Low & Medium & High & Low & 100 & 0 & 0 \\
\hline Winter & Medium & Low & Low & Medium & High & Medium & 100 & 0 & 0 \\
\hline Winter & Medium & Low & Low & Medium & High & High & 100 & 0 & 0 \\
\hline Winter & Medium & Low & Low & High & Low & Low & 100 & 0 & 0 \\
\hline Winter & Medium & Low & Low & High & Low & Medium & 100 & 0 & 0 \\
\hline Winter & Medium & Low & Low & High & Low & High & 100 & 0 & 0 \\
\hline Winter & Medium & Low & Low & High & Medium & Low & 100 & 0 & 0 \\
\hline
\end{tabular}




\begin{tabular}{|c|c|c|c|c|c|c|c|c|c|}
\hline \multicolumn{7}{|c|}{ Inputs } & \multicolumn{3}{|c|}{ Habitat quality } \\
\hline $\begin{array}{c}\text { Water } \\
\text { availability }\end{array}$ & Emergent & Floating & Terrestrial & $\begin{array}{l}\text { Winter } \\
\text { refuge }\end{array}$ & Submerged & $\begin{array}{l}\text { Water } \\
\text { quality }\end{array}$ & Low & Average & High \\
\hline Winter & Medium & Low & Low & High & Medium & Medium & 100 & 0 & 0 \\
\hline Winter & Medium & Low & Low & High & Medium & High & 100 & 0 & 0 \\
\hline Winter & Medium & Low & Low & High & High & Low & 100 & 0 & 0 \\
\hline Winter & Medium & Low & Low & High & High & Medium & 100 & 0 & 0 \\
\hline Winter & Medium & Low & Low & High & High & High & 100 & 0 & 0 \\
\hline Winter & Medium & Low & Medium & Low & Low & Low & 100 & 0 & 0 \\
\hline Winter & Medium & Low & Medium & Low & Low & Medium & 100 & 0 & 0 \\
\hline Winter & Medium & Low & Medium & Low & Low & High & 100 & 0 & 0 \\
\hline Winter & Medium & Low & Medium & Low & Medium & Low & 100 & 0 & 0 \\
\hline Winter & Medium & Low & Medium & Low & Medium & Medium & 100 & 0 & 0 \\
\hline Winter & Medium & Low & Medium & Low & Medium & High & 100 & 0 & 0 \\
\hline Winter & Medium & Low & Medium & Low & High & Low & 100 & 0 & 0 \\
\hline Winter & Medium & Low & Medium & Low & High & Medium & 100 & 0 & 0 \\
\hline Winter & Medium & Low & Medium & Low & High & High & 100 & 0 & 0 \\
\hline Winter & Medium & Low & Medium & Medium & Low & Low & 100 & 0 & 0 \\
\hline Winter & Medium & Low & Medium & Medium & Low & Medium & 100 & 0 & 0 \\
\hline Winter & Medium & Low & Medium & Medium & Low & High & 100 & 0 & 0 \\
\hline Winter & Medium & Low & Medium & Medium & Medium & Low & 100 & 0 & 0 \\
\hline Winter & Medium & Low & Medium & Medium & Medium & Medium & 100 & 0 & 0 \\
\hline Winter & Medium & Low & Medium & Medium & Medium & High & 100 & 0 & 0 \\
\hline Winter & Medium & Low & Medium & Medium & High & Low & 100 & 0 & 0 \\
\hline Winter & Medium & Low & Medium & Medium & High & Medium & 100 & 0 & 0 \\
\hline Winter & Medium & Low & Medium & Medium & High & High & 100 & 0 & 0 \\
\hline Winter & Medium & Low & Medium & High & Low & Low & 100 & 0 & 0 \\
\hline Winter & Medium & Low & Medium & High & Low & Medium & 100 & 0 & 0 \\
\hline Winter & Medium & Low & Medium & High & Low & High & 100 & 0 & 0 \\
\hline Winter & Medium & Low & Medium & High & Medium & Low & 100 & 0 & 0 \\
\hline Winter & Medium & Low & Medium & High & Medium & Medium & 100 & 0 & 0 \\
\hline Winter & Medium & Low & Medium & High & Medium & High & 100 & 0 & 0 \\
\hline Winter & Medium & Low & Medium & High & High & Low & 100 & 0 & 0 \\
\hline Winter & Medium & Low & Medium & High & High & Medium & 100 & 0 & 0 \\
\hline Winter & Medium & Low & Medium & High & High & High & 100 & 0 & 0 \\
\hline Winter & Medium & Low & High & Low & Low & Low & 100 & 0 & 0 \\
\hline Winter & Medium & Low & High & Low & Low & Medium & 100 & 0 & 0 \\
\hline Winter & Medium & Low & High & Low & Low & High & 100 & 0 & 0 \\
\hline Winter & Medium & Low & High & Low & Medium & Low & 100 & 0 & 0 \\
\hline Winter & Medium & Low & High & Low & Medium & Medium & 100 & 0 & 0 \\
\hline Winter & Medium & Low & High & Low & Medium & High & 100 & 0 & 0 \\
\hline Winter & Medium & Low & High & Low & High & Low & 100 & 0 & 0 \\
\hline Winter & Medium & Low & High & Low & High & Medium & 100 & 0 & 0 \\
\hline
\end{tabular}




\begin{tabular}{|c|c|c|c|c|c|c|c|c|c|}
\hline \multicolumn{7}{|c|}{ Inputs } & \multicolumn{3}{|c|}{ Habitat quality } \\
\hline $\begin{array}{c}\text { Water } \\
\text { availability }\end{array}$ & Emergent & Floating & Terrestrial & $\begin{array}{l}\text { Winter } \\
\text { refuge }\end{array}$ & Submerged & $\begin{array}{l}\text { Water } \\
\text { quality }\end{array}$ & Low & Average & High \\
\hline Winter & Medium & Low & High & Low & High & High & 100 & 0 & 0 \\
\hline Winter & Medium & Low & High & Medium & Low & Low & 100 & 0 & 0 \\
\hline Winter & Medium & Low & High & Medium & Low & Medium & 100 & 0 & 0 \\
\hline Winter & Medium & Low & High & Medium & Low & High & 100 & 0 & 0 \\
\hline Winter & Medium & Low & High & Medium & Medium & Low & 100 & 0 & 0 \\
\hline Winter & Medium & Low & High & Medium & Medium & Medium & 100 & 0 & 0 \\
\hline Winter & Medium & Low & High & Medium & Medium & High & 100 & 0 & 0 \\
\hline Winter & Medium & Low & High & Medium & High & Low & 100 & 0 & 0 \\
\hline Winter & Medium & Low & High & Medium & High & Medium & 100 & 0 & 0 \\
\hline Winter & Medium & Low & High & Medium & High & High & 100 & 0 & 0 \\
\hline Winter & Medium & Low & High & High & Low & Low & 100 & 0 & 0 \\
\hline Winter & Medium & Low & High & High & Low & Medium & 100 & 0 & 0 \\
\hline Winter & Medium & Low & High & High & Low & High & 100 & 0 & 0 \\
\hline Winter & Medium & Low & High & High & Medium & Low & 100 & 0 & 0 \\
\hline Winter & Medium & Low & High & High & Medium & Medium & 100 & 0 & 0 \\
\hline Winter & Medium & Low & High & High & Medium & High & 100 & 0 & 0 \\
\hline Winter & Medium & Low & High & High & High & Low & 100 & 0 & 0 \\
\hline Winter & Medium & Low & High & High & High & Medium & 100 & 0 & 0 \\
\hline Winter & Medium & Low & High & High & High & High & 100 & 0 & 0 \\
\hline Winter & Medium & Medium & Low & Low & Low & Low & 100 & 0 & 0 \\
\hline Winter & Medium & Medium & Low & Low & Low & Medium & 100 & 0 & 0 \\
\hline Winter & Medium & Medium & Low & Low & Low & High & 100 & 0 & 0 \\
\hline Winter & Medium & Medium & Low & Low & Medium & Low & 100 & 0 & 0 \\
\hline Winter & Medium & Medium & Low & Low & Medium & Medium & 100 & 0 & 0 \\
\hline Winter & Medium & Medium & Low & Low & Medium & High & 100 & 0 & 0 \\
\hline Winter & Medium & Medium & Low & Low & High & Low & 100 & 0 & 0 \\
\hline Winter & Medium & Medium & Low & Low & High & Medium & 100 & 0 & 0 \\
\hline Winter & Medium & Medium & Low & Low & High & High & 100 & 0 & 0 \\
\hline Winter & Medium & Medium & Low & Medium & Low & Low & 100 & 0 & 0 \\
\hline Winter & Medium & Medium & Low & Medium & Low & Medium & 100 & 0 & 0 \\
\hline Winter & Medium & Medium & Low & Medium & Low & High & 100 & 0 & 0 \\
\hline Winter & Medium & Medium & Low & Medium & Medium & Low & 100 & 0 & 0 \\
\hline Winter & Medium & Medium & Low & Medium & Medium & Medium & 100 & 0 & 0 \\
\hline Winter & Medium & Medium & Low & Medium & Medium & High & 100 & 0 & 0 \\
\hline Winter & Medium & Medium & Low & Medium & High & Low & 100 & 0 & 0 \\
\hline Winter & Medium & Medium & Low & Medium & High & Medium & 100 & 0 & 0 \\
\hline Winter & Medium & Medium & Low & Medium & High & High & 100 & 0 & 0 \\
\hline Winter & Medium & Medium & Low & High & Low & Low & 100 & 0 & 0 \\
\hline Winter & Medium & Medium & Low & High & Low & Medium & 100 & 0 & 0 \\
\hline Winter & Medium & Medium & Low & High & Low & High & 100 & 0 & 0 \\
\hline
\end{tabular}




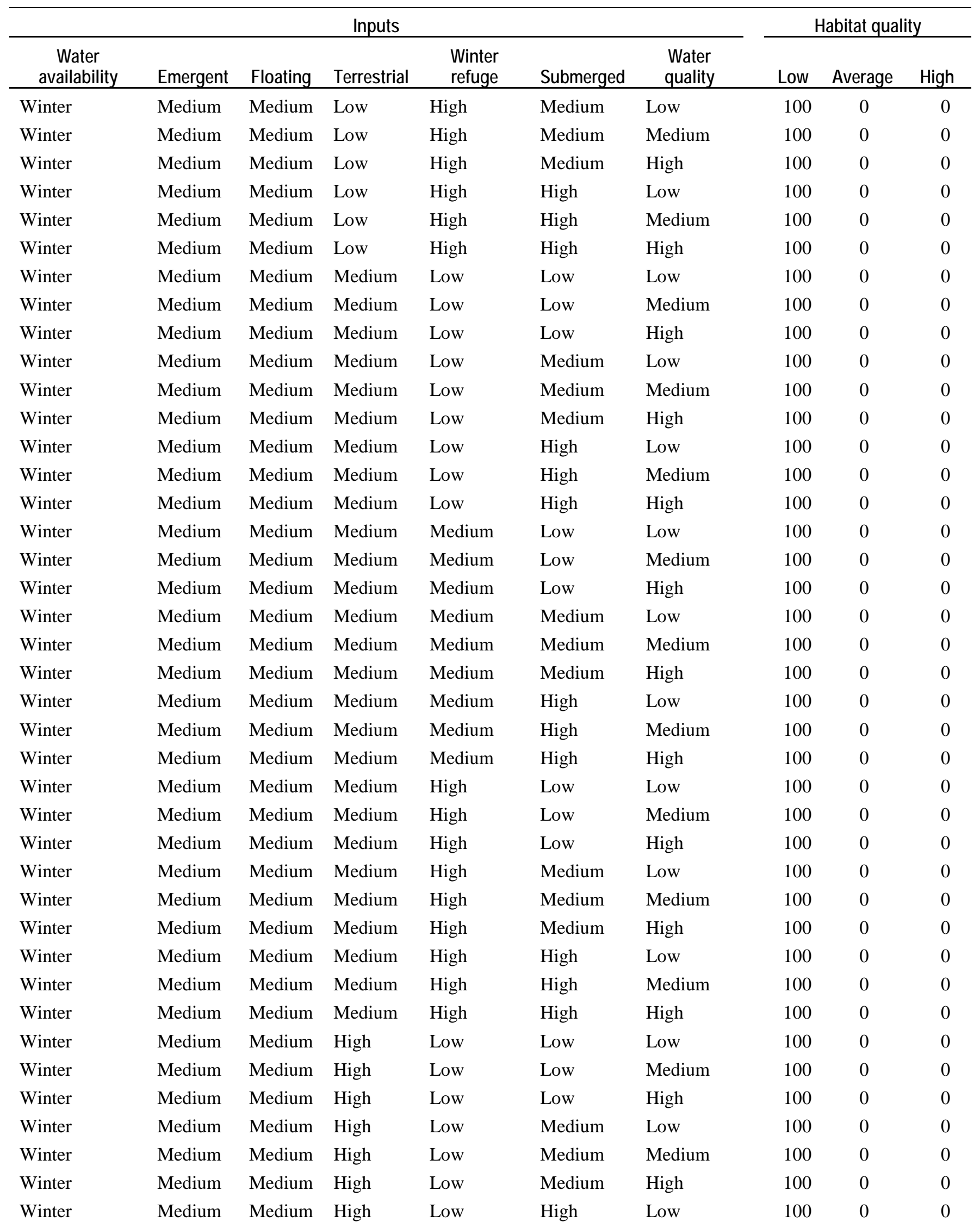




\begin{tabular}{|c|c|c|c|c|c|c|c|c|c|}
\hline \multicolumn{7}{|c|}{ Inputs } & \multicolumn{3}{|c|}{ Habitat quality } \\
\hline $\begin{array}{c}\text { Water } \\
\text { availability }\end{array}$ & Emergent & Floating & Terrestrial & $\begin{array}{l}\text { Winter } \\
\text { refuge }\end{array}$ & Submerged & $\begin{array}{l}\text { Water } \\
\text { quality }\end{array}$ & Low & Average & High \\
\hline Winter & Medium & Medium & High & Low & High & Medium & 100 & 0 & 0 \\
\hline Winter & Medium & Medium & High & Low & High & High & 100 & 0 & 0 \\
\hline Winter & Medium & Medium & High & Medium & Low & Low & 100 & 0 & 0 \\
\hline Winter & Medium & Medium & High & Medium & Low & Medium & 100 & 0 & 0 \\
\hline Winter & Medium & Medium & High & Medium & Low & High & 100 & 0 & 0 \\
\hline Winter & Medium & Medium & High & Medium & Medium & Low & 100 & 0 & 0 \\
\hline Winter & Medium & Medium & High & Medium & Medium & Medium & 100 & 0 & 0 \\
\hline Winter & Medium & Medium & High & Medium & Medium & High & 100 & 0 & 0 \\
\hline Winter & Medium & Medium & High & Medium & High & Low & 100 & 0 & 0 \\
\hline Winter & Medium & Medium & High & Medium & High & Medium & 100 & 0 & 0 \\
\hline Winter & Medium & Medium & High & Medium & High & High & 100 & 0 & 0 \\
\hline Winter & Medium & Medium & High & High & Low & Low & 100 & 0 & 0 \\
\hline Winter & Medium & Medium & High & High & Low & Medium & 100 & 0 & 0 \\
\hline Winter & Medium & Medium & High & High & Low & High & 100 & 0 & 0 \\
\hline Winter & Medium & Medium & High & High & Medium & Low & 100 & 0 & 0 \\
\hline Winter & Medium & Medium & High & High & Medium & Medium & 100 & 0 & 0 \\
\hline Winter & Medium & Medium & High & High & Medium & High & 100 & 0 & 0 \\
\hline Winter & Medium & Medium & High & High & High & Low & 100 & 0 & 0 \\
\hline Winter & Medium & Medium & High & High & High & Medium & 100 & 0 & 0 \\
\hline Winter & Medium & Medium & High & High & High & High & 100 & 0 & 0 \\
\hline Winter & Medium & High & Low & Low & Low & Low & 100 & 0 & 0 \\
\hline Winter & Medium & High & Low & Low & Low & Medium & 100 & 0 & 0 \\
\hline Winter & Medium & High & Low & Low & Low & High & 100 & 0 & 0 \\
\hline Winter & Medium & High & Low & Low & Medium & Low & 100 & 0 & 0 \\
\hline Winter & Medium & High & Low & Low & Medium & Medium & 100 & 0 & 0 \\
\hline Winter & Medium & High & Low & Low & Medium & High & 100 & 0 & 0 \\
\hline Winter & Medium & High & Low & Low & High & Low & 100 & 0 & 0 \\
\hline Winter & Medium & High & Low & Low & High & Medium & 100 & 0 & 0 \\
\hline Winter & Medium & High & Low & Low & High & High & 100 & 0 & 0 \\
\hline Winter & Medium & High & Low & Medium & Low & Low & 100 & 0 & 0 \\
\hline Winter & Medium & High & Low & Medium & Low & Medium & 100 & 0 & 0 \\
\hline Winter & Medium & High & Low & Medium & Low & High & 100 & 0 & 0 \\
\hline Winter & Medium & High & Low & Medium & Medium & Low & 100 & 0 & 0 \\
\hline Winter & Medium & High & Low & Medium & Medium & Medium & 100 & 0 & 0 \\
\hline Winter & Medium & High & Low & Medium & Medium & High & 100 & 0 & 0 \\
\hline Winter & Medium & High & Low & Medium & High & Low & 100 & 0 & 0 \\
\hline Winter & Medium & High & Low & Medium & High & Medium & 100 & 0 & 0 \\
\hline Winter & Medium & High & Low & Medium & High & High & 100 & 0 & 0 \\
\hline Winter & Medium & High & Low & High & Low & Low & 100 & 0 & 0 \\
\hline Winter & Medium & High & Low & High & Low & Medium & 100 & 0 & 0 \\
\hline
\end{tabular}




\begin{tabular}{|c|c|c|c|c|c|c|c|c|c|}
\hline \multicolumn{7}{|c|}{ Inputs } & \multicolumn{3}{|c|}{ Habitat quality } \\
\hline $\begin{array}{c}\text { Water } \\
\text { availability }\end{array}$ & Emergent & Floating & Terrestrial & $\begin{array}{l}\text { Winter } \\
\text { refuge }\end{array}$ & Submerged & $\begin{array}{l}\text { Water } \\
\text { quality }\end{array}$ & Low & Average & High \\
\hline Winter & Medium & High & Low & High & Low & High & 100 & 0 & 0 \\
\hline Winter & Medium & High & Low & High & Medium & Low & 100 & 0 & 0 \\
\hline Winter & Medium & High & Low & High & Medium & Medium & 100 & 0 & 0 \\
\hline Winter & Medium & High & Low & High & Medium & High & 100 & 0 & 0 \\
\hline Winter & Medium & High & Low & High & High & Low & 100 & 0 & 0 \\
\hline Winter & Medium & High & Low & High & High & Medium & 100 & 0 & 0 \\
\hline Winter & Medium & High & Low & High & High & High & 100 & 0 & 0 \\
\hline Winter & Medium & High & Medium & Low & Low & Low & 100 & 0 & 0 \\
\hline Winter & Medium & High & Medium & Low & Low & Medium & 100 & 0 & 0 \\
\hline Winter & Medium & High & Medium & Low & Low & High & 100 & 0 & 0 \\
\hline Winter & Medium & High & Medium & Low & Medium & Low & 100 & 0 & 0 \\
\hline Winter & Medium & High & Medium & Low & Medium & Medium & 100 & 0 & 0 \\
\hline Winter & Medium & High & Medium & Low & Medium & High & 100 & 0 & 0 \\
\hline Winter & Medium & High & Medium & Low & High & Low & 100 & 0 & 0 \\
\hline Winter & Medium & High & Medium & Low & High & Medium & 100 & 0 & 0 \\
\hline Winter & Medium & High & Medium & Low & High & High & 100 & 0 & 0 \\
\hline Winter & Medium & High & Medium & Medium & Low & Low & 100 & 0 & 0 \\
\hline Winter & Medium & High & Medium & Medium & Low & Medium & 100 & 0 & 0 \\
\hline Winter & Medium & High & Medium & Medium & Low & High & 100 & 0 & 0 \\
\hline Winter & Medium & High & Medium & Medium & Medium & Low & 100 & 0 & 0 \\
\hline Winter & Medium & High & Medium & Medium & Medium & Medium & 100 & 0 & 0 \\
\hline Winter & Medium & High & Medium & Medium & Medium & High & 100 & 0 & 0 \\
\hline Winter & Medium & High & Medium & Medium & High & Low & 100 & 0 & 0 \\
\hline Winter & Medium & High & Medium & Medium & High & Medium & 100 & 0 & 0 \\
\hline Winter & Medium & High & Medium & Medium & High & High & 100 & 0 & 0 \\
\hline Winter & Medium & High & Medium & High & Low & Low & 100 & 0 & 0 \\
\hline Winter & Medium & High & Medium & High & Low & Medium & 100 & 0 & 0 \\
\hline Winter & Medium & High & Medium & High & Low & High & 100 & 0 & 0 \\
\hline Winter & Medium & High & Medium & High & Medium & Low & 100 & 0 & 0 \\
\hline Winter & Medium & High & Medium & High & Medium & Medium & 100 & 0 & 0 \\
\hline Winter & Medium & High & Medium & High & Medium & High & 100 & 0 & 0 \\
\hline Winter & Medium & High & Medium & High & High & Low & 100 & 0 & 0 \\
\hline Winter & Medium & High & Medium & High & High & Medium & 100 & 0 & 0 \\
\hline Winter & Medium & High & Medium & High & High & High & 100 & 0 & 0 \\
\hline Winter & Medium & High & High & Low & Low & Low & 100 & 0 & 0 \\
\hline Winter & Medium & High & High & Low & Low & Medium & 100 & 0 & 0 \\
\hline Winter & Medium & High & High & Low & Low & High & 100 & 0 & 0 \\
\hline Winter & Medium & High & High & Low & Medium & Low & 100 & 0 & 0 \\
\hline Winter & Medium & High & High & Low & Medium & Medium & 100 & 0 & 0 \\
\hline Winter & Medium & High & High & Low & Medium & High & 100 & 0 & 0 \\
\hline
\end{tabular}




\begin{tabular}{|c|c|c|c|c|c|c|c|c|c|}
\hline \multicolumn{7}{|c|}{ Inputs } & \multicolumn{3}{|c|}{ Habitat quality } \\
\hline $\begin{array}{c}\text { Water } \\
\text { availability }\end{array}$ & Emergent & Floating & Terrestrial & $\begin{array}{l}\text { Winter } \\
\text { refuge }\end{array}$ & Submerged & $\begin{array}{l}\text { Water } \\
\text { quality }\end{array}$ & Low & Average & High \\
\hline Winter & Medium & High & High & Low & High & Low & 100 & 0 & 0 \\
\hline Winter & Medium & High & High & Low & High & Medium & 100 & 0 & 0 \\
\hline Winter & Medium & High & High & Low & High & High & 100 & 0 & 0 \\
\hline Winter & Medium & High & High & Medium & Low & Low & 100 & 0 & 0 \\
\hline Winter & Medium & High & High & Medium & Low & Medium & 100 & 0 & 0 \\
\hline Winter & Medium & High & High & Medium & Low & High & 100 & 0 & 0 \\
\hline Winter & Medium & High & High & Medium & Medium & Low & 100 & 0 & 0 \\
\hline Winter & Medium & High & High & Medium & Medium & Medium & 100 & 0 & 0 \\
\hline Winter & Medium & High & High & Medium & Medium & High & 100 & 0 & 0 \\
\hline Winter & Medium & High & High & Medium & High & Low & 100 & 0 & 0 \\
\hline Winter & Medium & High & High & Medium & High & Medium & 100 & 0 & 0 \\
\hline Winter & Medium & High & High & Medium & High & High & 100 & 0 & 0 \\
\hline Winter & Medium & High & High & High & Low & Low & 100 & 0 & 0 \\
\hline Winter & Medium & High & High & High & Low & Medium & 100 & 0 & 0 \\
\hline Winter & Medium & High & High & High & Low & High & 100 & 0 & 0 \\
\hline Winter & Medium & High & High & High & Medium & Low & 100 & 0 & 0 \\
\hline Winter & Medium & High & High & High & Medium & Medium & 100 & 0 & 0 \\
\hline Winter & Medium & High & High & High & Medium & High & 100 & 0 & 0 \\
\hline Winter & Medium & High & High & High & High & Low & 100 & 0 & 0 \\
\hline Winter & Medium & High & High & High & High & Medium & 100 & 0 & 0 \\
\hline Winter & Medium & High & High & High & High & High & 100 & 0 & 0 \\
\hline Winter & High & Low & Low & Low & Low & Low & 100 & 0 & 0 \\
\hline Winter & High & Low & Low & Low & Low & Medium & 100 & 0 & 0 \\
\hline Winter & High & Low & Low & Low & Low & High & 100 & 0 & 0 \\
\hline Winter & High & Low & Low & Low & Medium & Low & 100 & 0 & 0 \\
\hline Winter & High & Low & Low & Low & Medium & Medium & 100 & 0 & 0 \\
\hline Winter & High & Low & Low & Low & Medium & High & 100 & 0 & 0 \\
\hline Winter & High & Low & Low & Low & High & Low & 100 & 0 & 0 \\
\hline Winter & High & Low & Low & Low & High & Medium & 100 & 0 & 0 \\
\hline Winter & High & Low & Low & Low & High & High & 100 & 0 & 0 \\
\hline Winter & High & Low & Low & Medium & Low & Low & 100 & 0 & 0 \\
\hline Winter & High & Low & Low & Medium & Low & Medium & 100 & 0 & 0 \\
\hline Winter & High & Low & Low & Medium & Low & High & 100 & 0 & 0 \\
\hline Winter & High & Low & Low & Medium & Medium & Low & 100 & 0 & 0 \\
\hline Winter & High & Low & Low & Medium & Medium & Medium & 100 & 0 & 0 \\
\hline Winter & High & Low & Low & Medium & Medium & High & 100 & 0 & 0 \\
\hline Winter & High & Low & Low & Medium & High & Low & 100 & 0 & 0 \\
\hline Winter & High & Low & Low & Medium & High & Medium & 100 & 0 & 0 \\
\hline Winter & High & Low & Low & Medium & High & High & 100 & 0 & 0 \\
\hline Winter & High & Low & Low & High & Low & Low & 100 & 0 & 0 \\
\hline
\end{tabular}




\begin{tabular}{|c|c|c|c|c|c|c|c|c|c|}
\hline \multicolumn{7}{|c|}{ Inputs } & \multicolumn{3}{|c|}{ Habitat quality } \\
\hline $\begin{array}{c}\text { Water } \\
\text { availability }\end{array}$ & Emergent & Floating & Terrestrial & $\begin{array}{l}\text { Winter } \\
\text { refuge }\end{array}$ & Submerged & $\begin{array}{l}\text { Water } \\
\text { quality }\end{array}$ & Low & Average & High \\
\hline Winter & High & Low & Low & High & Low & Medium & 100 & 0 & 0 \\
\hline Winter & High & Low & Low & High & Low & High & 100 & 0 & 0 \\
\hline Winter & High & Low & Low & High & Medium & Low & 100 & 0 & 0 \\
\hline Winter & High & Low & Low & High & Medium & Medium & 100 & 0 & 0 \\
\hline Winter & High & Low & Low & High & Medium & High & 100 & 0 & 0 \\
\hline Winter & High & Low & Low & High & High & Low & 100 & 0 & 0 \\
\hline Winter & High & Low & Low & High & High & Medium & 100 & 0 & 0 \\
\hline Winter & High & Low & Low & High & High & High & 100 & 0 & 0 \\
\hline Winter & High & Low & Medium & Low & Low & Low & 100 & 0 & 0 \\
\hline Winter & High & Low & Medium & Low & Low & Medium & 100 & 0 & 0 \\
\hline Winter & High & Low & Medium & Low & Low & High & 100 & 0 & 0 \\
\hline Winter & High & Low & Medium & Low & Medium & Low & 100 & 0 & 0 \\
\hline Winter & High & Low & Medium & Low & Medium & Medium & 100 & 0 & 0 \\
\hline Winter & High & Low & Medium & Low & Medium & High & 100 & 0 & 0 \\
\hline Winter & High & Low & Medium & Low & High & Low & 100 & 0 & 0 \\
\hline Winter & High & Low & Medium & Low & High & Medium & 100 & 0 & 0 \\
\hline Winter & High & Low & Medium & Low & High & High & 100 & 0 & 0 \\
\hline Winter & High & Low & Medium & Medium & Low & Low & 100 & 0 & 0 \\
\hline Winter & High & Low & Medium & Medium & Low & Medium & 100 & 0 & 0 \\
\hline Winter & High & Low & Medium & Medium & Low & High & 100 & 0 & 0 \\
\hline Winter & High & Low & Medium & Medium & Medium & Low & 100 & 0 & 0 \\
\hline Winter & High & Low & Medium & Medium & Medium & Medium & 100 & 0 & 0 \\
\hline Winter & High & Low & Medium & Medium & Medium & High & 100 & 0 & 0 \\
\hline Winter & High & Low & Medium & Medium & High & Low & 100 & 0 & 0 \\
\hline Winter & High & Low & Medium & Medium & High & Medium & 100 & 0 & 0 \\
\hline Winter & High & Low & Medium & Medium & High & High & 100 & 0 & 0 \\
\hline Winter & High & Low & Medium & High & Low & Low & 100 & 0 & 0 \\
\hline Winter & High & Low & Medium & High & Low & Medium & 100 & 0 & 0 \\
\hline Winter & High & Low & Medium & High & Low & High & 100 & 0 & 0 \\
\hline Winter & High & Low & Medium & High & Medium & Low & 100 & 0 & 0 \\
\hline Winter & High & Low & Medium & High & Medium & Medium & 100 & 0 & 0 \\
\hline Winter & High & Low & Medium & High & Medium & High & 100 & 0 & 0 \\
\hline Winter & High & Low & Medium & High & High & Low & 100 & 0 & 0 \\
\hline Winter & High & Low & Medium & High & High & Medium & 100 & 0 & 0 \\
\hline Winter & High & Low & Medium & High & High & High & 100 & 0 & 0 \\
\hline Winter & High & Low & High & Low & Low & Low & 100 & 0 & 0 \\
\hline Winter & High & Low & High & Low & Low & Medium & 100 & 0 & 0 \\
\hline Winter & High & Low & High & Low & Low & High & 100 & 0 & 0 \\
\hline Winter & High & Low & High & Low & Medium & Low & 100 & 0 & 0 \\
\hline Winter & High & Low & High & Low & Medium & Medium & 100 & 0 & 0 \\
\hline
\end{tabular}




\begin{tabular}{|c|c|c|c|c|c|c|c|c|c|}
\hline \multicolumn{7}{|c|}{ Inputs } & \multicolumn{3}{|c|}{ Habitat quality } \\
\hline $\begin{array}{c}\text { Water } \\
\text { availability }\end{array}$ & Emergent & Floating & Terrestrial & $\begin{array}{l}\text { Winter } \\
\text { refuge }\end{array}$ & Submerged & $\begin{array}{l}\text { Water } \\
\text { quality }\end{array}$ & Low & Average & High \\
\hline Winter & High & Low & High & Low & Medium & High & 100 & 0 & 0 \\
\hline Winter & High & Low & High & Low & High & Low & 100 & 0 & 0 \\
\hline Winter & High & Low & High & Low & High & Medium & 100 & 0 & 0 \\
\hline Winter & High & Low & High & Low & High & High & 100 & 0 & 0 \\
\hline Winter & High & Low & High & Medium & Low & Low & 100 & 0 & 0 \\
\hline Winter & High & Low & High & Medium & Low & Medium & 100 & 0 & 0 \\
\hline Winter & High & Low & High & Medium & Low & High & 100 & 0 & 0 \\
\hline Winter & High & Low & High & Medium & Medium & Low & 100 & 0 & 0 \\
\hline Winter & High & Low & High & Medium & Medium & Medium & 100 & 0 & 0 \\
\hline Winter & High & Low & High & Medium & Medium & High & 100 & 0 & 0 \\
\hline Winter & High & Low & High & Medium & High & Low & 100 & 0 & 0 \\
\hline Winter & High & Low & High & Medium & High & Medium & 100 & 0 & 0 \\
\hline Winter & High & Low & High & Medium & High & High & 100 & 0 & 0 \\
\hline Winter & High & Low & High & High & Low & Low & 100 & 0 & 0 \\
\hline Winter & High & Low & High & High & Low & Medium & 100 & 0 & 0 \\
\hline Winter & High & Low & High & High & Low & High & 100 & 0 & 0 \\
\hline Winter & High & Low & High & High & Medium & Low & 100 & 0 & 0 \\
\hline Winter & High & Low & High & High & Medium & Medium & 100 & 0 & 0 \\
\hline Winter & High & Low & High & High & Medium & High & 100 & 0 & 0 \\
\hline Winter & High & Low & High & High & High & Low & 100 & 0 & 0 \\
\hline Winter & High & Low & High & High & High & Medium & 100 & 0 & 0 \\
\hline Winter & High & Low & High & High & High & High & 100 & 0 & 0 \\
\hline Winter & High & Medium & Low & Low & Low & Low & 100 & 0 & 0 \\
\hline Winter & High & Medium & Low & Low & Low & Medium & 100 & 0 & 0 \\
\hline Winter & High & Medium & Low & Low & Low & High & 100 & 0 & 0 \\
\hline Winter & High & Medium & Low & Low & Medium & Low & 100 & 0 & 0 \\
\hline Winter & High & Medium & Low & Low & Medium & Medium & 100 & 0 & 0 \\
\hline Winter & High & Medium & Low & Low & Medium & High & 100 & 0 & 0 \\
\hline Winter & High & Medium & Low & Low & High & Low & 100 & 0 & 0 \\
\hline Winter & High & Medium & Low & Low & High & Medium & 100 & 0 & 0 \\
\hline Winter & High & Medium & Low & Low & High & High & 100 & 0 & 0 \\
\hline Winter & High & Medium & Low & Medium & Low & Low & 100 & 0 & 0 \\
\hline Winter & High & Medium & Low & Medium & Low & Medium & 100 & 0 & 0 \\
\hline Winter & High & Medium & Low & Medium & Low & High & 100 & 0 & 0 \\
\hline Winter & High & Medium & Low & Medium & Medium & Low & 100 & 0 & 0 \\
\hline Winter & High & Medium & Low & Medium & Medium & Medium & 100 & 0 & 0 \\
\hline Winter & High & Medium & Low & Medium & Medium & High & 100 & 0 & 0 \\
\hline Winter & High & Medium & Low & Medium & High & Low & 100 & 0 & 0 \\
\hline Winter & High & Medium & Low & Medium & High & Medium & 100 & 0 & 0 \\
\hline Winter & High & Medium & Low & Medium & High & High & 100 & 0 & 0 \\
\hline
\end{tabular}




\begin{tabular}{|c|c|c|c|c|c|c|c|c|c|}
\hline \multicolumn{7}{|c|}{ Inputs } & \multicolumn{3}{|c|}{ Habitat quality } \\
\hline $\begin{array}{c}\text { Water } \\
\text { availability }\end{array}$ & Emergent & Floating & Terrestrial & $\begin{array}{l}\text { Winter } \\
\text { refuge }\end{array}$ & Submerged & $\begin{array}{l}\text { Water } \\
\text { quality }\end{array}$ & Low & Average & High \\
\hline Winter & High & Medium & Low & High & Low & Low & 100 & 0 & 0 \\
\hline Winter & High & Medium & Low & High & Low & Medium & 100 & 0 & 0 \\
\hline Winter & High & Medium & Low & High & Low & High & 100 & 0 & 0 \\
\hline Winter & High & Medium & Low & High & Medium & Low & 100 & 0 & 0 \\
\hline Winter & High & Medium & Low & High & Medium & Medium & 100 & 0 & 0 \\
\hline Winter & High & Medium & Low & High & Medium & High & 100 & 0 & 0 \\
\hline Winter & High & Medium & Low & High & High & Low & 100 & 0 & 0 \\
\hline Winter & High & Medium & Low & High & High & Medium & 100 & 0 & 0 \\
\hline Winter & High & Medium & Low & High & High & High & 100 & 0 & 0 \\
\hline Winter & High & Medium & Medium & Low & Low & Low & 100 & 0 & 0 \\
\hline Winter & High & Medium & Medium & Low & Low & Medium & 100 & 0 & 0 \\
\hline Winter & High & Medium & Medium & Low & Low & High & 100 & 0 & 0 \\
\hline Winter & High & Medium & Medium & Low & Medium & Low & 100 & 0 & 0 \\
\hline Winter & High & Medium & Medium & Low & Medium & Medium & 100 & 0 & 0 \\
\hline Winter & High & Medium & Medium & Low & Medium & High & 100 & 0 & 0 \\
\hline Winter & High & Medium & Medium & Low & High & Low & 100 & 0 & 0 \\
\hline Winter & High & Medium & Medium & Low & High & Medium & 100 & 0 & 0 \\
\hline Winter & High & Medium & Medium & Low & High & High & 100 & 0 & 0 \\
\hline Winter & High & Medium & Medium & Medium & Low & Low & 100 & 0 & 0 \\
\hline Winter & High & Medium & Medium & Medium & Low & Medium & 100 & 0 & 0 \\
\hline Winter & High & Medium & Medium & Medium & Low & High & 100 & 0 & 0 \\
\hline Winter & High & Medium & Medium & Medium & Medium & Low & 100 & 0 & 0 \\
\hline Winter & High & Medium & Medium & Medium & Medium & Medium & 100 & 0 & 0 \\
\hline Winter & High & Medium & Medium & Medium & Medium & High & 100 & 0 & 0 \\
\hline Winter & High & Medium & Medium & Medium & High & Low & 100 & 0 & 0 \\
\hline Winter & High & Medium & Medium & Medium & High & Medium & 100 & 0 & 0 \\
\hline Winter & High & Medium & Medium & Medium & High & High & 100 & 0 & 0 \\
\hline Winter & High & Medium & Medium & High & Low & Low & 100 & 0 & 0 \\
\hline Winter & High & Medium & Medium & High & Low & Medium & 100 & 0 & 0 \\
\hline Winter & High & Medium & Medium & High & Low & High & 100 & 0 & 0 \\
\hline Winter & High & Medium & Medium & High & Medium & Low & 100 & 0 & 0 \\
\hline Winter & High & Medium & Medium & High & Medium & Medium & 100 & 0 & 0 \\
\hline Winter & High & Medium & Medium & High & Medium & High & 100 & 0 & 0 \\
\hline Winter & High & Medium & Medium & High & High & Low & 100 & 0 & 0 \\
\hline Winter & High & Medium & Medium & High & High & Medium & 100 & 0 & 0 \\
\hline Winter & High & Medium & Medium & High & High & High & 100 & 0 & 0 \\
\hline Winter & High & Medium & High & Low & Low & Low & 100 & 0 & 0 \\
\hline Winter & High & Medium & High & Low & Low & Medium & 100 & 0 & 0 \\
\hline Winter & High & Medium & High & Low & Low & High & 100 & 0 & 0 \\
\hline Winter & High & Medium & High & Low & Medium & Low & 100 & 0 & 0 \\
\hline
\end{tabular}




\begin{tabular}{|c|c|c|c|c|c|c|c|c|c|}
\hline \multicolumn{7}{|c|}{ Inputs } & \multicolumn{3}{|c|}{ Habitat quality } \\
\hline $\begin{array}{c}\text { Water } \\
\text { availability }\end{array}$ & Emergent & Floating & Terrestrial & $\begin{array}{l}\text { Winter } \\
\text { refuge }\end{array}$ & Submerged & $\begin{array}{l}\text { Water } \\
\text { quality }\end{array}$ & Low & Average & High \\
\hline Winter & High & Medium & High & Low & Medium & Medium & 100 & 0 & 0 \\
\hline Winter & High & Medium & High & Low & Medium & High & 100 & 0 & 0 \\
\hline Winter & High & Medium & High & Low & High & Low & 100 & 0 & 0 \\
\hline Winter & High & Medium & High & Low & High & Medium & 100 & 0 & 0 \\
\hline Winter & High & Medium & High & Low & High & High & 100 & 0 & 0 \\
\hline Winter & High & Medium & High & Medium & Low & Low & 100 & 0 & 0 \\
\hline Winter & High & Medium & High & Medium & Low & Medium & 100 & 0 & 0 \\
\hline Winter & High & Medium & High & Medium & Low & High & 100 & 0 & 0 \\
\hline Winter & High & Medium & High & Medium & Medium & Low & 100 & 0 & 0 \\
\hline Winter & High & Medium & High & Medium & Medium & Medium & 100 & 0 & 0 \\
\hline Winter & High & Medium & High & Medium & Medium & High & 100 & 0 & 0 \\
\hline Winter & High & Medium & High & Medium & High & Low & 100 & 0 & 0 \\
\hline Winter & High & Medium & High & Medium & High & Medium & 100 & 0 & 0 \\
\hline Winter & High & Medium & High & Medium & High & High & 100 & 0 & 0 \\
\hline Winter & High & Medium & High & High & Low & Low & 100 & 0 & 0 \\
\hline Winter & High & Medium & High & High & Low & Medium & 100 & 0 & 0 \\
\hline Winter & High & Medium & High & High & Low & High & 100 & 0 & 0 \\
\hline Winter & High & Medium & High & High & Medium & Low & 100 & 0 & 0 \\
\hline Winter & High & Medium & High & High & Medium & Medium & 100 & 0 & 0 \\
\hline Winter & High & Medium & High & High & Medium & High & 100 & 0 & 0 \\
\hline Winter & High & Medium & High & High & High & Low & 100 & 0 & 0 \\
\hline Winter & High & Medium & High & High & High & Medium & 100 & 0 & 0 \\
\hline Winter & High & Medium & High & High & High & High & 100 & 0 & 0 \\
\hline Winter & High & High & Low & Low & Low & Low & 100 & 0 & 0 \\
\hline Winter & High & High & Low & Low & Low & Medium & 100 & 0 & 0 \\
\hline Winter & High & High & Low & Low & Low & High & 100 & 0 & 0 \\
\hline Winter & High & High & Low & Low & Medium & Low & 100 & 0 & 0 \\
\hline Winter & High & High & Low & Low & Medium & Medium & 100 & 0 & 0 \\
\hline Winter & High & High & Low & Low & Medium & High & 100 & 0 & 0 \\
\hline Winter & High & High & Low & Low & High & Low & 100 & 0 & 0 \\
\hline Winter & High & High & Low & Low & High & Medium & 100 & 0 & 0 \\
\hline Winter & High & High & Low & Low & High & High & 100 & 0 & 0 \\
\hline Winter & High & High & Low & Medium & Low & Low & 100 & 0 & 0 \\
\hline Winter & High & High & Low & Medium & Low & Medium & 100 & 0 & 0 \\
\hline Winter & High & High & Low & Medium & Low & High & 100 & 0 & 0 \\
\hline Winter & High & High & Low & Medium & Medium & Low & 100 & 0 & 0 \\
\hline Winter & High & High & Low & Medium & Medium & Medium & 100 & 0 & 0 \\
\hline Winter & High & High & Low & Medium & Medium & High & 100 & 0 & 0 \\
\hline Winter & High & High & Low & Medium & High & Low & 100 & 0 & 0 \\
\hline Winter & High & High & Low & Medium & High & Medium & 100 & 0 & 0 \\
\hline
\end{tabular}




\begin{tabular}{|c|c|c|c|c|c|c|c|c|c|}
\hline \multicolumn{7}{|c|}{ Inputs } & \multicolumn{3}{|c|}{ Habitat quality } \\
\hline $\begin{array}{c}\text { Water } \\
\text { availability }\end{array}$ & Emergent & Floating & Terrestrial & $\begin{array}{l}\text { Winter } \\
\text { refuge }\end{array}$ & Submerged & $\begin{array}{l}\text { Water } \\
\text { quality }\end{array}$ & Low & Average & High \\
\hline Winter & High & High & Low & Medium & High & High & 100 & 0 & 0 \\
\hline Winter & High & High & Low & High & Low & Low & 100 & 0 & 0 \\
\hline Winter & High & High & Low & High & Low & Medium & 100 & 0 & 0 \\
\hline Winter & High & High & Low & High & Low & High & 100 & 0 & 0 \\
\hline Winter & High & High & Low & High & Medium & Low & 100 & 0 & 0 \\
\hline Winter & High & High & Low & High & Medium & Medium & 100 & 0 & 0 \\
\hline Winter & High & High & Low & High & Medium & High & 100 & 0 & 0 \\
\hline Winter & High & High & Low & High & High & Low & 100 & 0 & 0 \\
\hline Winter & High & High & Low & High & High & Medium & 100 & 0 & 0 \\
\hline Winter & High & High & Low & High & High & High & 100 & 0 & 0 \\
\hline Winter & High & High & Medium & Low & Low & Low & 100 & 0 & 0 \\
\hline Winter & High & High & Medium & Low & Low & Medium & 100 & 0 & 0 \\
\hline Winter & High & High & Medium & Low & Low & High & 100 & 0 & 0 \\
\hline Winter & High & High & Medium & Low & Medium & Low & 100 & 0 & 0 \\
\hline Winter & High & High & Medium & Low & Medium & Medium & 100 & 0 & 0 \\
\hline Winter & High & High & Medium & Low & Medium & High & 100 & 0 & 0 \\
\hline Winter & High & High & Medium & Low & High & Low & 100 & 0 & 0 \\
\hline Winter & High & High & Medium & Low & High & Medium & 100 & 0 & 0 \\
\hline Winter & High & High & Medium & Low & High & High & 100 & 0 & 0 \\
\hline Winter & High & High & Medium & Medium & Low & Low & 100 & 0 & 0 \\
\hline Winter & High & High & Medium & Medium & Low & Medium & 100 & 0 & 0 \\
\hline Winter & High & High & Medium & Medium & Low & High & 100 & 0 & 0 \\
\hline Winter & High & High & Medium & Medium & Medium & Low & 100 & 0 & 0 \\
\hline Winter & High & High & Medium & Medium & Medium & Medium & 100 & 0 & 0 \\
\hline Winter & High & High & Medium & Medium & Medium & High & 100 & 0 & 0 \\
\hline Winter & High & High & Medium & Medium & High & Low & 100 & 0 & 0 \\
\hline Winter & High & High & Medium & Medium & High & Medium & 100 & 0 & 0 \\
\hline Winter & High & High & Medium & Medium & High & High & 100 & 0 & 0 \\
\hline Winter & High & High & Medium & High & Low & Low & 100 & 0 & 0 \\
\hline Winter & High & High & Medium & High & Low & Medium & 100 & 0 & 0 \\
\hline Winter & High & High & Medium & High & Low & High & 100 & 0 & 0 \\
\hline Winter & High & High & Medium & High & Medium & Low & 100 & 0 & 0 \\
\hline Winter & High & High & Medium & High & Medium & Medium & 100 & 0 & 0 \\
\hline Winter & High & High & Medium & High & Medium & High & 100 & 0 & 0 \\
\hline Winter & High & High & Medium & High & High & Low & 100 & 0 & 0 \\
\hline Winter & High & High & Medium & High & High & Medium & 100 & 0 & 0 \\
\hline Winter & High & High & Medium & High & High & High & 100 & 0 & 0 \\
\hline Winter & High & High & High & Low & Low & Low & 100 & 0 & 0 \\
\hline Winter & High & High & High & Low & Low & Medium & 100 & 0 & 0 \\
\hline Winter & High & High & High & Low & Low & High & 100 & 0 & 0 \\
\hline
\end{tabular}




\begin{tabular}{|c|c|c|c|c|c|c|c|c|c|}
\hline \multicolumn{7}{|c|}{ Inputs } & \multicolumn{3}{|c|}{ Habitat quality } \\
\hline $\begin{array}{c}\text { Water } \\
\text { availability }\end{array}$ & Emergent & Floating & Terrestrial & $\begin{array}{l}\text { Winter } \\
\text { refuge }\end{array}$ & Submerged & $\begin{array}{l}\text { Water } \\
\text { quality }\end{array}$ & Low & Average & High \\
\hline Winter & High & High & High & Low & Medium & Low & 100 & 0 & 0 \\
\hline Winter & High & High & High & Low & Medium & Medium & 100 & 0 & 0 \\
\hline Winter & High & High & High & Low & Medium & High & 100 & 0 & 0 \\
\hline Winter & High & High & High & Low & High & Low & 100 & 0 & 0 \\
\hline Winter & High & High & High & Low & High & Medium & 100 & 0 & 0 \\
\hline Winter & High & High & High & Low & High & High & 100 & 0 & 0 \\
\hline Winter & High & High & High & Medium & Low & Low & 100 & 0 & 0 \\
\hline Winter & High & High & High & Medium & Low & Medium & 100 & 0 & 0 \\
\hline Winter & High & High & High & Medium & Low & High & 100 & 0 & 0 \\
\hline Winter & High & High & High & Medium & Medium & Low & 100 & 0 & 0 \\
\hline Winter & High & High & High & Medium & Medium & Medium & 100 & 0 & 0 \\
\hline Winter & High & High & High & Medium & Medium & High & 100 & 0 & 0 \\
\hline Winter & High & High & High & Medium & High & Low & 100 & 0 & 0 \\
\hline Winter & High & High & High & Medium & High & Medium & 100 & 0 & 0 \\
\hline Winter & High & High & High & Medium & High & High & 100 & 0 & 0 \\
\hline Winter & High & High & High & High & Low & Low & 100 & 0 & 0 \\
\hline Winter & High & High & High & High & Low & Medium & 100 & 0 & 0 \\
\hline Winter & High & High & High & High & Low & High & 100 & 0 & 0 \\
\hline Winter & High & High & High & High & Medium & Low & 100 & 0 & 0 \\
\hline Winter & High & High & High & High & Medium & Medium & 100 & 0 & 0 \\
\hline Winter & High & High & High & High & Medium & High & 100 & 0 & 0 \\
\hline Winter & High & High & High & High & High & Low & 100 & 0 & 0 \\
\hline Winter & High & High & High & High & High & Medium & 100 & 0 & 0 \\
\hline Winter & High & High & High & High & High & High & 100 & 0 & 0 \\
\hline Groundwater & Low & Low & Low & Low & Low & Low & 45 & 55 & 0 \\
\hline Groundwater & Low & Low & Low & Low & Low & Medium & 35 & 55 & 10 \\
\hline Groundwater & Low & Low & Low & Low & Low & High & 25 & 55 & 20 \\
\hline Groundwater & Low & Low & Low & Low & Medium & Low & 50 & 50 & 0 \\
\hline Groundwater & Low & Low & Low & Low & Medium & Medium & 40 & 60 & 0 \\
\hline Groundwater & Low & Low & Low & Low & Medium & High & 30 & 60 & 10 \\
\hline Groundwater & Low & Low & Low & Low & High & Low & 65 & 35 & 0 \\
\hline Groundwater & Low & Low & Low & Low & High & Medium & 50 & 50 & 0 \\
\hline Groundwater & Low & Low & Low & Low & High & High & 40 & 60 & 0 \\
\hline Groundwater & Low & Low & Low & Medium & Low & Low & 40 & 60 & 0 \\
\hline Groundwater & Low & Low & Low & Medium & Low & Medium & 30 & 50 & 20 \\
\hline Groundwater & Low & Low & Low & Medium & Low & High & 20 & 50 & 30 \\
\hline Groundwater & Low & Low & Low & Medium & Medium & Low & 45 & 55 & 0 \\
\hline Groundwater & Low & Low & Low & Medium & Medium & Medium & 35 & 55 & 10 \\
\hline Groundwater & Low & Low & Low & Medium & Medium & High & 25 & 55 & 20 \\
\hline Groundwater & Low & Low & Low & Medium & High & Low & 65 & 35 & 0 \\
\hline
\end{tabular}




\begin{tabular}{|c|c|c|c|c|c|c|c|c|c|}
\hline \multicolumn{7}{|c|}{ Inputs } & \multicolumn{3}{|c|}{ Habitat quality } \\
\hline $\begin{array}{c}\text { Water } \\
\text { availability }\end{array}$ & Emergent & Floating & Terrestrial & $\begin{array}{l}\text { Winter } \\
\text { refuge }\end{array}$ & Submerged & $\begin{array}{l}\text { Water } \\
\text { quality }\end{array}$ & Low & Average & High \\
\hline Groundwater & Low & Low & Low & Medium & High & Medium & 45 & 55 & 0 \\
\hline Groundwater & Low & Low & Low & Medium & High & High & 35 & 65 & 0 \\
\hline Groundwater & Low & Low & Low & High & Low & Low & 40 & 55 & 5 \\
\hline Groundwater & Low & Low & Low & High & Low & Medium & 30 & 45 & 25 \\
\hline Groundwater & Low & Low & Low & High & Low & High & 20 & 45 & 35 \\
\hline Groundwater & Low & Low & Low & High & Medium & Low & 45 & 55 & 0 \\
\hline Groundwater & Low & Low & Low & High & Medium & Medium & 35 & 50 & 15 \\
\hline Groundwater & Low & Low & Low & High & Medium & High & 25 & 50 & 25 \\
\hline Groundwater & Low & Low & Low & High & High & Low & 60 & 40 & 0 \\
\hline Groundwater & Low & Low & Low & High & High & Medium & 45 & 55 & 0 \\
\hline Groundwater & Low & Low & Low & High & High & High & 35 & 60 & 5 \\
\hline Groundwater & Low & Low & Medium & Low & Low & Low & 40 & 60 & 0 \\
\hline Groundwater & Low & Low & Medium & Low & Low & Medium & 30 & 50 & 20 \\
\hline Groundwater & Low & Low & Medium & Low & Low & High & 20 & 50 & 30 \\
\hline Groundwater & Low & Low & Medium & Low & Medium & Low & 45 & 55 & 0 \\
\hline Groundwater & Low & Low & Medium & Low & Medium & Medium & 35 & 55 & 10 \\
\hline Groundwater & Low & Low & Medium & Low & Medium & High & 25 & 55 & 20 \\
\hline Groundwater & Low & Low & Medium & Low & High & Low & 65 & 35 & 0 \\
\hline Groundwater & Low & Low & Medium & Low & High & Medium & 45 & 55 & 0 \\
\hline Groundwater & Low & Low & Medium & Low & High & High & 35 & 65 & 0 \\
\hline Groundwater & Low & Low & Medium & Medium & Low & Low & 35 & 55 & 10 \\
\hline Groundwater & Low & Low & Medium & Medium & Low & Medium & 25 & 45 & 30 \\
\hline Groundwater & Low & Low & Medium & Medium & Low & High & 15 & 45 & 40 \\
\hline Groundwater & Low & Low & Medium & Medium & Medium & Low & 40 & 60 & 0 \\
\hline Groundwater & Low & Low & Medium & Medium & Medium & Medium & 30 & 50 & 20 \\
\hline Groundwater & Low & Low & Medium & Medium & Medium & High & 20 & 50 & 30 \\
\hline Groundwater & Low & Low & Medium & Medium & High & Low & 60 & 40 & 0 \\
\hline Groundwater & Low & Low & Medium & Medium & High & Medium & 40 & 60 & 0 \\
\hline Groundwater & Low & Low & Medium & Medium & High & High & 30 & 60 & 10 \\
\hline Groundwater & Low & Low & Medium & High & Low & Low & 35 & 50 & 15 \\
\hline Groundwater & Low & Low & Medium & High & Low & Medium & 25 & 40 & 35 \\
\hline Groundwater & Low & Low & Medium & High & Low & High & 15 & 40 & 45 \\
\hline Groundwater & Low & Low & Medium & High & Medium & Low & 40 & 55 & 5 \\
\hline Groundwater & Low & Low & Medium & High & Medium & Medium & 30 & 45 & 25 \\
\hline Groundwater & Low & Low & Medium & High & Medium & High & 20 & 45 & 35 \\
\hline Groundwater & Low & Low & Medium & High & High & Low & 55 & 45 & 0 \\
\hline Groundwater & Low & Low & Medium & High & High & Medium & 40 & 55 & 5 \\
\hline Groundwater & Low & Low & Medium & High & High & High & 30 & 55 & 15 \\
\hline Groundwater & Low & Low & High & Low & Low & Low & 40 & 55 & 5 \\
\hline Groundwater & Low & Low & High & Low & Low & Medium & 30 & 45 & 25 \\
\hline
\end{tabular}




\begin{tabular}{|c|c|c|c|c|c|c|c|c|c|}
\hline \multicolumn{7}{|c|}{ Inputs } & \multicolumn{3}{|c|}{ Habitat quality } \\
\hline $\begin{array}{c}\text { Water } \\
\text { availability }\end{array}$ & Emergent & Floating & Terrestrial & $\begin{array}{l}\text { Winter } \\
\text { refuge }\end{array}$ & Submerged & $\begin{array}{l}\text { Water } \\
\text { quality }\end{array}$ & Low & Average & High \\
\hline Groundwater & Low & Low & High & Low & Low & High & 20 & 45 & 35 \\
\hline Groundwater & Low & Low & High & Low & Medium & Low & 45 & 55 & 0 \\
\hline Groundwater & Low & Low & High & Low & Medium & Medium & 35 & 50 & 15 \\
\hline Groundwater & Low & Low & High & Low & Medium & High & 25 & 50 & 25 \\
\hline Groundwater & Low & Low & High & Low & High & Low & 60 & 40 & 0 \\
\hline Groundwater & Low & Low & High & Low & High & Medium & 45 & 55 & 0 \\
\hline Groundwater & Low & Low & High & Low & High & High & 35 & 60 & 5 \\
\hline Groundwater & Low & Low & High & Medium & Low & Low & 35 & 50 & 15 \\
\hline Groundwater & Low & Low & High & Medium & Low & Medium & 25 & 40 & 35 \\
\hline Groundwater & Low & Low & High & Medium & Low & High & 15 & 40 & 45 \\
\hline Groundwater & Low & Low & High & Medium & Medium & Low & 40 & 55 & 5 \\
\hline Groundwater & Low & Low & High & Medium & Medium & Medium & 30 & 45 & 25 \\
\hline Groundwater & Low & Low & High & Medium & Medium & High & 20 & 45 & 35 \\
\hline Groundwater & Low & Low & High & Medium & High & Low & 55 & 45 & 0 \\
\hline Groundwater & Low & Low & High & Medium & High & Medium & 40 & 55 & 5 \\
\hline Groundwater & Low & Low & High & Medium & High & High & 30 & 55 & 15 \\
\hline Groundwater & Low & Low & High & High & Low & Low & 35 & 45 & 20 \\
\hline Groundwater & Low & Low & High & High & Low & Medium & 25 & 35 & 40 \\
\hline Groundwater & Low & Low & High & High & Low & High & 15 & 35 & 50 \\
\hline Groundwater & Low & Low & High & High & Medium & Low & 40 & 50 & 10 \\
\hline Groundwater & Low & Low & High & High & Medium & Medium & 30 & 40 & 30 \\
\hline Groundwater & Low & Low & High & High & Medium & High & 20 & 40 & 40 \\
\hline Groundwater & Low & Low & High & High & High & Low & 50 & 50 & 0 \\
\hline Groundwater & Low & Low & High & High & High & Medium & 40 & 50 & 10 \\
\hline Groundwater & Low & Low & High & High & High & High & 30 & 50 & 20 \\
\hline Groundwater & Low & Medium & Low & Low & Low & Low & 50 & 50 & 0 \\
\hline Groundwater & Low & Medium & Low & Low & Low & Medium & 40 & 55 & 5 \\
\hline Groundwater & Low & Medium & Low & Low & Low & High & 30 & 55 & 15 \\
\hline Groundwater & Low & Medium & Low & Low & Medium & Low & 55 & 45 & 0 \\
\hline Groundwater & Low & Medium & Low & Low & Medium & Medium & 45 & 55 & 0 \\
\hline Groundwater & Low & Medium & Low & Low & Medium & High & 35 & 60 & 5 \\
\hline Groundwater & Low & Medium & Low & Low & High & Low & 70 & 30 & 0 \\
\hline Groundwater & Low & Medium & Low & Low & High & Medium & 55 & 45 & 0 \\
\hline Groundwater & Low & Medium & Low & Low & High & High & 45 & 55 & 0 \\
\hline Groundwater & Low & Medium & Low & Medium & Low & Low & 45 & 55 & 0 \\
\hline Groundwater & Low & Medium & Low & Medium & Low & Medium & 35 & 50 & 15 \\
\hline Groundwater & Low & Medium & Low & Medium & Low & High & 25 & 50 & 25 \\
\hline Groundwater & Low & Medium & Low & Medium & Medium & Low & 50 & 50 & 0 \\
\hline Groundwater & Low & Medium & Low & Medium & Medium & Medium & 40 & 55 & 5 \\
\hline Groundwater & Low & Medium & Low & Medium & Medium & High & 30 & 55 & 15 \\
\hline
\end{tabular}




\begin{tabular}{|c|c|c|c|c|c|c|c|c|c|}
\hline \multicolumn{7}{|c|}{ Inputs } & \multicolumn{3}{|c|}{ Habitat quality } \\
\hline $\begin{array}{c}\text { Water } \\
\text { availability }\end{array}$ & Emergent & Floating & Terrestrial & $\begin{array}{l}\text { Winter } \\
\text { refuge }\end{array}$ & Submerged & $\begin{array}{l}\text { Water } \\
\text { quality }\end{array}$ & Low & Average & High \\
\hline Groundwater & Low & Medium & Low & Medium & High & Low & 70 & 30 & 0 \\
\hline Groundwater & Low & Medium & Low & Medium & High & Medium & 50 & 50 & 0 \\
\hline Groundwater & Low & Medium & Low & Medium & High & High & 40 & 60 & 0 \\
\hline Groundwater & Low & Medium & Low & High & Low & Low & 45 & 55 & 0 \\
\hline Groundwater & Low & Medium & Low & High & Low & Medium & 35 & 45 & 20 \\
\hline Groundwater & Low & Medium & Low & High & Low & High & 25 & 45 & 30 \\
\hline Groundwater & Low & Medium & Low & High & Medium & Low & 50 & 50 & 0 \\
\hline Groundwater & Low & Medium & Low & High & Medium & Medium & 40 & 50 & 10 \\
\hline Groundwater & Low & Medium & Low & High & Medium & High & 30 & 50 & 20 \\
\hline Groundwater & Low & Medium & Low & High & High & Low & 65 & 35 & 0 \\
\hline Groundwater & Low & Medium & Low & High & High & Medium & 50 & 50 & 0 \\
\hline Groundwater & Low & Medium & Low & High & High & High & 40 & 60 & 0 \\
\hline Groundwater & Low & Medium & Medium & Low & Low & Low & 45 & 55 & 0 \\
\hline Groundwater & Low & Medium & Medium & Low & Low & Medium & 35 & 50 & 15 \\
\hline Groundwater & Low & Medium & Medium & Low & Low & High & 25 & 50 & 25 \\
\hline Groundwater & Low & Medium & Medium & Low & Medium & Low & 50 & 50 & 0 \\
\hline Groundwater & Low & Medium & Medium & Low & Medium & Medium & 40 & 55 & 5 \\
\hline Groundwater & Low & Medium & Medium & Low & Medium & High & 30 & 55 & 15 \\
\hline Groundwater & Low & Medium & Medium & Low & High & Low & 70 & 30 & 0 \\
\hline Groundwater & Low & Medium & Medium & Low & High & Medium & 50 & 50 & 0 \\
\hline Groundwater & Low & Medium & Medium & Low & High & High & 40 & 60 & 0 \\
\hline Groundwater & Low & Medium & Medium & Medium & Low & Low & 40 & 55 & 5 \\
\hline Groundwater & Low & Medium & Medium & Medium & Low & Medium & 30 & 45 & 25 \\
\hline Groundwater & Low & Medium & Medium & Medium & Low & High & 20 & 45 & 35 \\
\hline Groundwater & Low & Medium & Medium & Medium & Medium & Low & 45 & 55 & 0 \\
\hline Groundwater & Low & Medium & Medium & Medium & Medium & Medium & 35 & 50 & 15 \\
\hline Groundwater & Low & Medium & Medium & Medium & Medium & High & 25 & 50 & 25 \\
\hline Groundwater & Low & Medium & Medium & Medium & High & Low & 65 & 35 & 0 \\
\hline Groundwater & Low & Medium & Medium & Medium & High & Medium & 45 & 55 & 0 \\
\hline Groundwater & Low & Medium & Medium & Medium & High & High & 35 & 60 & 5 \\
\hline Groundwater & Low & Medium & Medium & High & Low & Low & 40 & 50 & 10 \\
\hline Groundwater & Low & Medium & Medium & High & Low & Medium & 30 & 40 & 30 \\
\hline Groundwater & Low & Medium & Medium & High & Low & High & 20 & 40 & 40 \\
\hline Groundwater & Low & Medium & Medium & High & Medium & Low & 45 & 55 & 0 \\
\hline Groundwater & Low & Medium & Medium & High & Medium & Medium & 35 & 45 & 20 \\
\hline Groundwater & Low & Medium & Medium & High & Medium & High & 25 & 45 & 30 \\
\hline Groundwater & Low & Medium & Medium & High & High & Low & 60 & 40 & 0 \\
\hline Groundwater & Low & Medium & Medium & High & High & Medium & 45 & 55 & 0 \\
\hline Groundwater & Low & Medium & Medium & High & High & High & 35 & 55 & 10 \\
\hline Groundwater & Low & Medium & High & Low & Low & Low & 45 & 55 & 0 \\
\hline
\end{tabular}




\begin{tabular}{|c|c|c|c|c|c|c|c|c|c|}
\hline \multicolumn{7}{|c|}{ Inputs } & \multicolumn{3}{|c|}{ Habitat quality } \\
\hline $\begin{array}{c}\text { Water } \\
\text { availability }\end{array}$ & Emergent & Floating & Terrestrial & $\begin{array}{l}\text { Winter } \\
\text { refuge }\end{array}$ & Submerged & $\begin{array}{l}\text { Water } \\
\text { quality }\end{array}$ & Low & Average & High \\
\hline Groundwater & Low & Medium & High & Low & Low & Medium & 35 & 45 & 20 \\
\hline Groundwater & Low & Medium & High & Low & Low & High & 25 & 45 & 30 \\
\hline Groundwater & Low & Medium & High & Low & Medium & Low & 50 & 50 & 0 \\
\hline Groundwater & Low & Medium & High & Low & Medium & Medium & 40 & 50 & 10 \\
\hline Groundwater & Low & Medium & High & Low & Medium & High & 30 & 50 & 20 \\
\hline Groundwater & Low & Medium & High & Low & High & Low & 65 & 35 & 0 \\
\hline Groundwater & Low & Medium & High & Low & High & Medium & 50 & 50 & 0 \\
\hline Groundwater & Low & Medium & High & Low & High & High & 40 & 60 & 0 \\
\hline Groundwater & Low & Medium & High & Medium & Low & Low & 40 & 50 & 10 \\
\hline Groundwater & Low & Medium & High & Medium & Low & Medium & 30 & 40 & 30 \\
\hline Groundwater & Low & Medium & High & Medium & Low & High & 20 & 40 & 40 \\
\hline Groundwater & Low & Medium & High & Medium & Medium & Low & 45 & 55 & 0 \\
\hline Groundwater & Low & Medium & High & Medium & Medium & Medium & 35 & 45 & 20 \\
\hline Groundwater & Low & Medium & High & Medium & Medium & High & 25 & 45 & 30 \\
\hline Groundwater & Low & Medium & High & Medium & High & Low & 60 & 40 & 0 \\
\hline Groundwater & Low & Medium & High & Medium & High & Medium & 45 & 55 & 0 \\
\hline Groundwater & Low & Medium & High & Medium & High & High & 35 & 55 & 10 \\
\hline Groundwater & Low & Medium & High & High & Low & Low & 40 & 45 & 15 \\
\hline Groundwater & Low & Medium & High & High & Low & Medium & 30 & 35 & 35 \\
\hline Groundwater & Low & Medium & High & High & Low & High & 20 & 35 & 45 \\
\hline Groundwater & Low & Medium & High & High & Medium & Low & 45 & 50 & 5 \\
\hline Groundwater & Low & Medium & High & High & Medium & Medium & 35 & 40 & 25 \\
\hline Groundwater & Low & Medium & High & High & Medium & High & 25 & 40 & 35 \\
\hline Groundwater & Low & Medium & High & High & High & Low & 55 & 45 & 0 \\
\hline Groundwater & Low & Medium & High & High & High & Medium & 45 & 50 & 5 \\
\hline Groundwater & Low & Medium & High & High & High & High & 35 & 50 & 15 \\
\hline Groundwater & Low & High & Low & Low & Low & Low & 55 & 45 & 0 \\
\hline Groundwater & Low & High & Low & Low & Low & Medium & 45 & 55 & 0 \\
\hline Groundwater & Low & High & Low & Low & Low & High & 35 & 60 & 5 \\
\hline Groundwater & Low & High & Low & Low & Medium & Low & 60 & 40 & 0 \\
\hline Groundwater & Low & High & Low & Low & Medium & Medium & 50 & 50 & 0 \\
\hline Groundwater & Low & High & Low & Low & Medium & High & 40 & 60 & 0 \\
\hline Groundwater & Low & High & Low & Low & High & Low & 75 & 25 & 0 \\
\hline Groundwater & Low & High & Low & Low & High & Medium & 60 & 40 & 0 \\
\hline Groundwater & Low & High & Low & Low & High & High & 50 & 50 & 0 \\
\hline Groundwater & Low & High & Low & Medium & Low & Low & 50 & 50 & 0 \\
\hline Groundwater & Low & High & Low & Medium & Low & Medium & 40 & 55 & 5 \\
\hline Groundwater & Low & High & Low & Medium & Low & High & 30 & 55 & 15 \\
\hline Groundwater & Low & High & Low & Medium & Medium & Low & 55 & 45 & 0 \\
\hline Groundwater & Low & High & Low & Medium & Medium & Medium & 45 & 55 & 0 \\
\hline
\end{tabular}




\begin{tabular}{|c|c|c|c|c|c|c|c|c|c|}
\hline \multicolumn{7}{|c|}{ Inputs } & \multicolumn{3}{|c|}{ Habitat quality } \\
\hline $\begin{array}{c}\text { Water } \\
\text { availability }\end{array}$ & Emergent & Floating & Terrestrial & $\begin{array}{l}\text { Winter } \\
\text { refuge }\end{array}$ & Submerged & $\begin{array}{l}\text { Water } \\
\text { quality }\end{array}$ & Low & Average & High \\
\hline Groundwater & Low & High & Low & Medium & Medium & High & 35 & 60 & 5 \\
\hline Groundwater & Low & High & Low & Medium & High & Low & 70 & 30 & 0 \\
\hline Groundwater & Low & High & Low & Medium & High & Medium & 55 & 45 & 0 \\
\hline Groundwater & Low & High & Low & Medium & High & High & 45 & 55 & 0 \\
\hline Groundwater & Low & High & Low & High & Low & Low & 50 & 50 & 0 \\
\hline Groundwater & Low & High & Low & High & Low & Medium & 40 & 50 & 10 \\
\hline Groundwater & Low & High & Low & High & Low & High & 30 & 50 & 20 \\
\hline Groundwater & Low & High & Low & High & Medium & Low & 55 & 45 & 0 \\
\hline Groundwater & Low & High & Low & High & Medium & Medium & 45 & 55 & 0 \\
\hline Groundwater & Low & High & Low & High & Medium & High & 35 & 55 & 10 \\
\hline Groundwater & Low & High & Low & High & High & Low & 65 & 35 & 0 \\
\hline Groundwater & Low & High & Low & High & High & Medium & 55 & 45 & 0 \\
\hline Groundwater & Low & High & Low & High & High & High & 45 & 55 & 0 \\
\hline Groundwater & Low & High & Medium & Low & Low & Low & 50 & 50 & 0 \\
\hline Groundwater & Low & High & Medium & Low & Low & Medium & 40 & 55 & 5 \\
\hline Groundwater & Low & High & Medium & Low & Low & High & 30 & 55 & 15 \\
\hline Groundwater & Low & High & Medium & Low & Medium & Low & 55 & 45 & 0 \\
\hline Groundwater & Low & High & Medium & Low & Medium & Medium & 45 & 55 & 0 \\
\hline Groundwater & Low & High & Medium & Low & Medium & High & 35 & 60 & 5 \\
\hline Groundwater & Low & High & Medium & Low & High & Low & 70 & 30 & 0 \\
\hline Groundwater & Low & High & Medium & Low & High & Medium & 55 & 45 & 0 \\
\hline Groundwater & Low & High & Medium & Low & High & High & 45 & 55 & 0 \\
\hline Groundwater & Low & High & Medium & Medium & Low & Low & 45 & 55 & 0 \\
\hline Groundwater & Low & High & Medium & Medium & Low & Medium & 35 & 50 & 15 \\
\hline Groundwater & Low & High & Medium & Medium & Low & High & 25 & 50 & 25 \\
\hline Groundwater & Low & High & Medium & Medium & Medium & Low & 50 & 50 & 0 \\
\hline Groundwater & Low & High & Medium & Medium & Medium & Medium & 40 & 55 & 5 \\
\hline Groundwater & Low & High & Medium & Medium & Medium & High & 30 & 55 & 15 \\
\hline Groundwater & Low & High & Medium & Medium & High & Low & 65 & 35 & 0 \\
\hline Groundwater & Low & High & Medium & Medium & High & Medium & 50 & 50 & 0 \\
\hline Groundwater & Low & High & Medium & Medium & High & High & 40 & 60 & 0 \\
\hline Groundwater & Low & High & Medium & High & Low & Low & 45 & 55 & 0 \\
\hline Groundwater & Low & High & Medium & High & Low & Medium & 35 & 45 & 20 \\
\hline Groundwater & Low & High & Medium & High & Low & High & 25 & 45 & 30 \\
\hline Groundwater & Low & High & Medium & High & Medium & Low & 50 & 50 & 0 \\
\hline Groundwater & Low & High & Medium & High & Medium & Medium & 40 & 50 & 10 \\
\hline Groundwater & Low & High & Medium & High & Medium & High & 30 & 50 & 20 \\
\hline Groundwater & Low & High & Medium & High & High & Low & 65 & 35 & 0 \\
\hline Groundwater & Low & High & Medium & High & High & Medium & 50 & 50 & 0 \\
\hline Groundwater & Low & High & Medium & High & High & High & 40 & 60 & 0 \\
\hline
\end{tabular}




\begin{tabular}{|c|c|c|c|c|c|c|c|c|c|}
\hline \multicolumn{7}{|c|}{ Inputs } & \multicolumn{3}{|c|}{ Habitat quality } \\
\hline $\begin{array}{c}\text { Water } \\
\text { availability }\end{array}$ & Emergent & Floating & Terrestrial & $\begin{array}{l}\text { Winter } \\
\text { refuge }\end{array}$ & Submerged & $\begin{array}{l}\text { Water } \\
\text { quality }\end{array}$ & Low & Average & High \\
\hline Groundwater & Low & High & High & Low & Low & Low & 50 & 50 & 0 \\
\hline Groundwater & Low & High & High & Low & Low & Medium & 40 & 50 & 10 \\
\hline Groundwater & Low & High & High & Low & Low & High & 30 & 50 & 20 \\
\hline Groundwater & Low & High & High & Low & Medium & Low & 55 & 45 & 0 \\
\hline Groundwater & Low & High & High & Low & Medium & Medium & 45 & 55 & 0 \\
\hline Groundwater & Low & High & High & Low & Medium & High & 35 & 55 & 10 \\
\hline Groundwater & Low & High & High & Low & High & Low & 65 & 35 & 0 \\
\hline Groundwater & Low & High & High & Low & High & Medium & 55 & 45 & 0 \\
\hline Groundwater & Low & High & High & Low & High & High & 45 & 55 & 0 \\
\hline Groundwater & Low & High & High & Medium & Low & Low & 45 & 55 & 0 \\
\hline Groundwater & Low & High & High & Medium & Low & Medium & 35 & 45 & 20 \\
\hline Groundwater & Low & High & High & Medium & Low & High & 25 & 45 & 30 \\
\hline Groundwater & Low & High & High & Medium & Medium & Low & 50 & 50 & 0 \\
\hline Groundwater & Low & High & High & Medium & Medium & Medium & 40 & 50 & 10 \\
\hline Groundwater & Low & High & High & Medium & Medium & High & 30 & 50 & 20 \\
\hline Groundwater & Low & High & High & Medium & High & Low & 65 & 35 & 0 \\
\hline Groundwater & Low & High & High & Medium & High & Medium & 50 & 50 & 0 \\
\hline Groundwater & Low & High & High & Medium & High & High & 40 & 60 & 0 \\
\hline Groundwater & Low & High & High & High & Low & Low & 45 & 50 & 5 \\
\hline Groundwater & Low & High & High & High & Low & Medium & 35 & 40 & 25 \\
\hline Groundwater & Low & High & High & High & Low & High & 25 & 40 & 35 \\
\hline Groundwater & Low & High & High & High & Medium & Low & 50 & 50 & 0 \\
\hline Groundwater & Low & High & High & High & Medium & Medium & 40 & 45 & 15 \\
\hline Groundwater & Low & High & High & High & Medium & High & 30 & 45 & 25 \\
\hline Groundwater & Low & High & High & High & High & Low & 60 & 40 & 0 \\
\hline Groundwater & Low & High & High & High & High & Medium & 50 & 50 & 0 \\
\hline Groundwater & Low & High & High & High & High & High & 40 & 55 & 5 \\
\hline Groundwater & Medium & Low & Low & Low & Low & Low & 40 & 60 & 0 \\
\hline Groundwater & Medium & Low & Low & Low & Low & Medium & 30 & 50 & 20 \\
\hline Groundwater & Medium & Low & Low & Low & Low & High & 20 & 50 & 30 \\
\hline Groundwater & Medium & Low & Low & Low & Medium & Low & 45 & 55 & 0 \\
\hline Groundwater & Medium & Low & Low & Low & Medium & Medium & 35 & 55 & 10 \\
\hline Groundwater & Medium & Low & Low & Low & Medium & High & 25 & 55 & 20 \\
\hline Groundwater & Medium & Low & Low & Low & High & Low & 65 & 35 & 0 \\
\hline Groundwater & Medium & Low & Low & Low & High & Medium & 45 & 55 & 0 \\
\hline Groundwater & Medium & Low & Low & Low & High & High & 35 & 65 & 0 \\
\hline Groundwater & Medium & Low & Low & Medium & Low & Low & 35 & 55 & 10 \\
\hline Groundwater & Medium & Low & Low & Medium & Low & Medium & 25 & 45 & 30 \\
\hline Groundwater & Medium & Low & Low & Medium & Low & High & 15 & 45 & 40 \\
\hline Groundwater & Medium & Low & Low & Medium & Medium & Low & 40 & 60 & 0 \\
\hline
\end{tabular}




\begin{tabular}{|c|c|c|c|c|c|c|c|c|c|}
\hline \multicolumn{7}{|c|}{ Inputs } & \multicolumn{3}{|c|}{ Habitat quality } \\
\hline $\begin{array}{c}\text { Water } \\
\text { availability }\end{array}$ & Emergent & Floating & Terrestrial & $\begin{array}{l}\text { Winter } \\
\text { refuge }\end{array}$ & Submerged & $\begin{array}{l}\text { Water } \\
\text { quality }\end{array}$ & Low & Average & High \\
\hline Groundwater & Medium & Low & Low & Medium & Medium & Medium & 30 & 50 & 20 \\
\hline Groundwater & Medium & Low & Low & Medium & Medium & High & 20 & 50 & 30 \\
\hline Groundwater & Medium & Low & Low & Medium & High & Low & 60 & 40 & 0 \\
\hline Groundwater & Medium & Low & Low & Medium & High & Medium & 40 & 60 & 0 \\
\hline Groundwater & Medium & Low & Low & Medium & High & High & 30 & 60 & 10 \\
\hline Groundwater & Medium & Low & Low & High & Low & Low & 35 & 50 & 15 \\
\hline Groundwater & Medium & Low & Low & High & Low & Medium & 25 & 40 & 35 \\
\hline Groundwater & Medium & Low & Low & High & Low & High & 15 & 40 & 45 \\
\hline Groundwater & Medium & Low & Low & High & Medium & Low & 40 & 55 & 5 \\
\hline Groundwater & Medium & Low & Low & High & Medium & Medium & 30 & 45 & 25 \\
\hline Groundwater & Medium & Low & Low & High & Medium & High & 20 & 45 & 35 \\
\hline Groundwater & Medium & Low & Low & High & High & Low & 55 & 45 & 0 \\
\hline Groundwater & Medium & Low & Low & High & High & Medium & 40 & 55 & 5 \\
\hline Groundwater & Medium & Low & Low & High & High & High & 30 & 55 & 15 \\
\hline Groundwater & Medium & Low & Medium & Low & Low & Low & 35 & 55 & 10 \\
\hline Groundwater & Medium & Low & Medium & Low & Low & Medium & 25 & 45 & 30 \\
\hline Groundwater & Medium & Low & Medium & Low & Low & High & 15 & 45 & 40 \\
\hline Groundwater & Medium & Low & Medium & Low & Medium & Low & 40 & 60 & 0 \\
\hline Groundwater & Medium & Low & Medium & Low & Medium & Medium & 30 & 50 & 20 \\
\hline Groundwater & Medium & Low & Medium & Low & Medium & High & 20 & 50 & 30 \\
\hline Groundwater & Medium & Low & Medium & Low & High & Low & 60 & 40 & 0 \\
\hline Groundwater & Medium & Low & Medium & Low & High & Medium & 40 & 60 & 0 \\
\hline Groundwater & Medium & Low & Medium & Low & High & High & 30 & 60 & 10 \\
\hline Groundwater & Medium & Low & Medium & Medium & Low & Low & 30 & 50 & 20 \\
\hline Groundwater & Medium & Low & Medium & Medium & Low & Medium & 20 & 40 & 40 \\
\hline Groundwater & Medium & Low & Medium & Medium & Low & High & 10 & 40 & 50 \\
\hline Groundwater & Medium & Low & Medium & Medium & Medium & Low & 35 & 55 & 10 \\
\hline Groundwater & Medium & Low & Medium & Medium & Medium & Medium & 25 & 45 & 30 \\
\hline Groundwater & Medium & Low & Medium & Medium & Medium & High & 15 & 45 & 40 \\
\hline Groundwater & Medium & Low & Medium & Medium & High & Low & 55 & 45 & 0 \\
\hline Groundwater & Medium & Low & Medium & Medium & High & Medium & 35 & 55 & 10 \\
\hline Groundwater & Medium & Low & Medium & Medium & High & High & 25 & 55 & 20 \\
\hline Groundwater & Medium & Low & Medium & High & Low & Low & 30 & 45 & 25 \\
\hline Groundwater & Medium & Low & Medium & High & Low & Medium & 20 & 35 & 45 \\
\hline Groundwater & Medium & Low & Medium & High & Low & High & 10 & 35 & 55 \\
\hline Groundwater & Medium & Low & Medium & High & Medium & Low & 35 & 50 & 15 \\
\hline Groundwater & Medium & Low & Medium & High & Medium & Medium & 25 & 40 & 35 \\
\hline Groundwater & Medium & Low & Medium & High & Medium & High & 15 & 40 & 45 \\
\hline Groundwater & Medium & Low & Medium & High & High & Low & 50 & 50 & 0 \\
\hline Groundwater & Medium & Low & Medium & High & High & Medium & 35 & 50 & 15 \\
\hline
\end{tabular}




\begin{tabular}{|c|c|c|c|c|c|c|c|c|c|}
\hline \multicolumn{7}{|c|}{ Inputs } & \multicolumn{3}{|c|}{ Habitat quality } \\
\hline $\begin{array}{c}\text { Water } \\
\text { availability }\end{array}$ & Emergent & Floating & Terrestrial & $\begin{array}{l}\text { Winter } \\
\text { refuge }\end{array}$ & Submerged & $\begin{array}{l}\text { Water } \\
\text { quality }\end{array}$ & Low & Average & High \\
\hline Groundwater & Medium & Low & Medium & High & High & High & 25 & 50 & 25 \\
\hline Groundwater & Medium & Low & High & Low & Low & Low & 35 & 50 & 15 \\
\hline Groundwater & Medium & Low & High & Low & Low & Medium & 25 & 40 & 35 \\
\hline Groundwater & Medium & Low & High & Low & Low & High & 15 & 40 & 45 \\
\hline Groundwater & Medium & Low & High & Low & Medium & Low & 40 & 55 & 5 \\
\hline Groundwater & Medium & Low & High & Low & Medium & Medium & 30 & 45 & 25 \\
\hline Groundwater & Medium & Low & High & Low & Medium & High & 20 & 45 & 35 \\
\hline Groundwater & Medium & Low & High & Low & High & Low & 55 & 45 & 0 \\
\hline Groundwater & Medium & Low & High & Low & High & Medium & 40 & 55 & 5 \\
\hline Groundwater & Medium & Low & High & Low & High & High & 30 & 55 & 15 \\
\hline Groundwater & Medium & Low & High & Medium & Low & Low & 30 & 45 & 25 \\
\hline Groundwater & Medium & Low & High & Medium & Low & Medium & 20 & 35 & 45 \\
\hline Groundwater & Medium & Low & High & Medium & Low & High & 10 & 35 & 55 \\
\hline Groundwater & Medium & Low & High & Medium & Medium & Low & 35 & 50 & 15 \\
\hline Groundwater & Medium & Low & High & Medium & Medium & Medium & 25 & 40 & 35 \\
\hline Groundwater & Medium & Low & High & Medium & Medium & High & 15 & 40 & 45 \\
\hline Groundwater & Medium & Low & High & Medium & High & Low & 50 & 50 & 0 \\
\hline Groundwater & Medium & Low & High & Medium & High & Medium & 35 & 50 & 15 \\
\hline Groundwater & Medium & Low & High & Medium & High & High & 25 & 50 & 25 \\
\hline Groundwater & Medium & Low & High & High & Low & Low & 30 & 40 & 30 \\
\hline Groundwater & Medium & Low & High & High & Low & Medium & 20 & 30 & 50 \\
\hline Groundwater & Medium & Low & High & High & Low & High & 10 & 30 & 60 \\
\hline Groundwater & Medium & Low & High & High & Medium & Low & 35 & 45 & 20 \\
\hline Groundwater & Medium & Low & High & High & Medium & Medium & 25 & 35 & 40 \\
\hline Groundwater & Medium & Low & High & High & Medium & High & 15 & 35 & 50 \\
\hline Groundwater & Medium & Low & High & High & High & Low & 45 & 55 & 0 \\
\hline Groundwater & Medium & Low & High & High & High & Medium & 35 & 45 & 20 \\
\hline Groundwater & Medium & Low & High & High & High & High & 25 & 45 & 30 \\
\hline Groundwater & Medium & Medium & Low & Low & Low & Low & 45 & 55 & 0 \\
\hline Groundwater & Medium & Medium & Low & Low & Low & Medium & 35 & 50 & 15 \\
\hline Groundwater & Medium & Medium & Low & Low & Low & High & 25 & 50 & 25 \\
\hline Groundwater & Medium & Medium & Low & Low & Medium & Low & 50 & 50 & 0 \\
\hline Groundwater & Medium & Medium & Low & Low & Medium & Medium & 40 & 55 & 5 \\
\hline Groundwater & Medium & Medium & Low & Low & Medium & High & 30 & 55 & 15 \\
\hline Groundwater & Medium & Medium & Low & Low & High & Low & 70 & 30 & 0 \\
\hline Groundwater & Medium & Medium & Low & Low & High & Medium & 50 & 50 & 0 \\
\hline Groundwater & Medium & Medium & Low & Low & High & High & 40 & 60 & 0 \\
\hline Groundwater & Medium & Medium & Low & Medium & Low & Low & 40 & 55 & 5 \\
\hline Groundwater & Medium & Medium & Low & Medium & Low & Medium & 30 & 45 & 25 \\
\hline Groundwater & Medium & Medium & Low & Medium & Low & High & 20 & 45 & 35 \\
\hline
\end{tabular}




\begin{tabular}{|c|c|c|c|c|c|c|c|c|c|}
\hline \multicolumn{7}{|c|}{ Inputs } & \multicolumn{3}{|c|}{ Habitat quality } \\
\hline Groundwater & Medium & Medium & Low & Medium & Medium & Medium & 35 & 50 & 15 \\
\hline Groundwater & Medium & Medium & Low & Medium & Medium & High & 25 & 50 & 25 \\
\hline Groundwater & Medium & Medium & Low & Medium & High & Medium & 45 & 55 & 0 \\
\hline Groundwater & Medium & Medium & Low & Medium & High & High & 35 & 60 & 5 \\
\hline Groundwater & Medium & Medium & Low & High & Low & Low & 40 & 50 & 10 \\
\hline Groundwater & Medium & Medium & Low & High & Low & Medium & 30 & 40 & 30 \\
\hline Groundwater & Medium & Medium & Low & High & Medium & High & 25 & 45 & 30 \\
\hline Groundwater & Medium & Medium & Low & High & High & Low & 60 & 40 & 0 \\
\hline Groundwater & Medium & Medium & Low & High & High & Medium & 45 & 55 & 0 \\
\hline Groundwater & Medium & Medium & Low & High & High & High & 35 & 55 & 10 \\
\hline Groundwater & Medium & Medium & Medium & Low & Low & Low & 40 & 55 & 5 \\
\hline Groundwater & Medium & Medium & Medium & Low & Low & Medium & 30 & 45 & 25 \\
\hline Groundwater & Medium & Medium & Medium & Low & Low & High & 20 & 45 & 35 \\
\hline Groundwater & Medium & Medium & Medium & Low & Medium & Low & 45 & 55 & 0 \\
\hline Groundwater & Medium & Medium & Medium & Medium & Low & Medium & 25 & 40 & 35 \\
\hline Groundwater & Medium & Medium & Medium & Medium & Low & High & 15 & 40 & 45 \\
\hline Groundwater & Medium & Medium & Medium & Medium & Medium & Low & 40 & 55 & 5 \\
\hline Groundwater & Medium & Medium & Medium & Medium & Medium & Medium & 30 & 45 & 25 \\
\hline Groundwater & Medium & Medium & Medium & Medium & Medium & High & 20 & 45 & 35 \\
\hline Groundwater & Medium & Medium & Medium & Medium & High & Low & 60 & 40 & 0 \\
\hline Groundwater & Medium & Medium & Medium & Medium & High & Medium & 40 & 55 & 5 \\
\hline Groundwater & Medium & Medium & Medium & Medium & High & High & 30 & 55 & 15 \\
\hline Groundwater & Medium & Medium & Medium & High & Low & Low & 35 & 45 & 20 \\
\hline Groundwater & Medium & Medium & Medium & High & Low & Medium & 25 & 35 & 40 \\
\hline Groundwater & Medium & Medium & Medium & High & Low & High & 15 & 35 & 50 \\
\hline Groundwater & Medium & Medium & Medium & High & Medium & Low & 40 & 50 & 10 \\
\hline Groundwater & Medium & Medium & Medium & High & Medium & Medium & 30 & 40 & 30 \\
\hline Groundwater & Medium & Medium & Medium & High & Medium & High & 20 & 40 & 40 \\
\hline Groundwater & Medium & Medium & Medium & High & High & Low & 55 & 45 & 0 \\
\hline
\end{tabular}




\begin{tabular}{|c|c|c|c|c|c|c|c|c|c|}
\hline \multicolumn{7}{|c|}{ Inputs } & \multicolumn{3}{|c|}{ Habitat quality } \\
\hline $\begin{array}{c}\text { Water } \\
\text { availability }\end{array}$ & Emergent & Floating & Terrestrial & $\begin{array}{l}\text { Winter } \\
\text { refuge }\end{array}$ & Submerged & $\begin{array}{l}\text { Water } \\
\text { quality }\end{array}$ & Low & Average & High \\
\hline Groundwater & Medium & Medium & Medium & High & High & Medium & 40 & 50 & 10 \\
\hline Groundwater & Medium & Medium & Medium & High & High & High & 30 & 50 & 20 \\
\hline Groundwater & Medium & Medium & High & Low & Low & Low & 40 & 50 & 10 \\
\hline Groundwater & Medium & Medium & High & Low & Low & Medium & 30 & 40 & 30 \\
\hline Groundwater & Medium & Medium & High & Low & Low & High & 20 & 40 & 40 \\
\hline Groundwater & Medium & Medium & High & Low & Medium & Low & 45 & 55 & 0 \\
\hline Groundwater & Medium & Medium & High & Low & Medium & Medium & 35 & 45 & 20 \\
\hline Groundwater & Medium & Medium & High & Low & Medium & High & 25 & 45 & 30 \\
\hline Groundwater & Medium & Medium & High & Low & High & Low & 60 & 40 & 0 \\
\hline Groundwater & Medium & Medium & High & Low & High & Medium & 45 & 55 & 0 \\
\hline Groundwater & Medium & Medium & High & Low & High & High & 35 & 55 & 10 \\
\hline Groundwater & Medium & Medium & High & Medium & Low & Low & 35 & 45 & 20 \\
\hline Groundwater & Medium & Medium & High & Medium & Low & Medium & 25 & 35 & 40 \\
\hline Groundwater & Medium & Medium & High & Medium & Low & High & 15 & 35 & 50 \\
\hline Groundwater & Medium & Medium & High & Medium & Medium & Low & 40 & 50 & 10 \\
\hline Groundwater & Medium & Medium & High & Medium & Medium & Medium & 30 & 40 & 30 \\
\hline Groundwater & Medium & Medium & High & Medium & Medium & High & 20 & 40 & 40 \\
\hline Groundwater & Medium & Medium & High & Medium & High & Low & 55 & 45 & 0 \\
\hline Groundwater & Medium & Medium & High & Medium & High & Medium & 40 & 50 & 10 \\
\hline Groundwater & Medium & Medium & High & Medium & High & High & 30 & 50 & 20 \\
\hline Groundwater & Medium & Medium & High & High & Low & Low & 35 & 40 & 25 \\
\hline Groundwater & Medium & Medium & High & High & Low & Medium & 25 & 30 & 45 \\
\hline Groundwater & Medium & Medium & High & High & Low & High & 15 & 30 & 55 \\
\hline Groundwater & Medium & Medium & High & High & Medium & Low & 40 & 45 & 15 \\
\hline Groundwater & Medium & Medium & High & High & Medium & Medium & 30 & 35 & 35 \\
\hline Groundwater & Medium & Medium & High & High & Medium & High & 20 & 35 & 45 \\
\hline Groundwater & Medium & Medium & High & High & High & Low & 50 & 50 & 0 \\
\hline Groundwater & Medium & Medium & High & High & High & Medium & 40 & 45 & 15 \\
\hline Groundwater & Medium & Medium & High & High & High & High & 30 & 45 & 25 \\
\hline Groundwater & Medium & High & Low & Low & Low & Low & 50 & 50 & 0 \\
\hline Groundwater & Medium & High & Low & Low & Low & Medium & 40 & 55 & 5 \\
\hline Groundwater & Medium & High & Low & Low & Low & High & 30 & 55 & 15 \\
\hline Groundwater & Medium & High & Low & Low & Medium & Low & 55 & 45 & 0 \\
\hline Groundwater & Medium & High & Low & Low & Medium & Medium & 45 & 50 & 5 \\
\hline Groundwater & Medium & High & Low & Low & Medium & High & 35 & 60 & 5 \\
\hline Groundwater & Medium & High & Low & Low & High & Low & 75 & 25 & 0 \\
\hline Groundwater & Medium & High & Low & Low & High & Medium & 55 & 45 & 0 \\
\hline Groundwater & Medium & High & Low & Low & High & High & 45 & 55 & 0 \\
\hline Groundwater & Medium & High & Low & Medium & Low & Low & 45 & 55 & 0 \\
\hline Groundwater & Medium & High & Low & Medium & Low & Medium & 35 & 50 & 15 \\
\hline
\end{tabular}




\begin{tabular}{|c|c|c|c|c|c|c|c|c|c|}
\hline \multicolumn{7}{|c|}{ Inputs } & \multicolumn{3}{|c|}{ Habitat quality } \\
\hline $\begin{array}{c}\text { Water } \\
\text { availability }\end{array}$ & Emergent & Floating & Terrestrial & $\begin{array}{l}\text { Winter } \\
\text { refuge }\end{array}$ & Submerged & $\begin{array}{l}\text { Water } \\
\text { quality }\end{array}$ & Low & Average & High \\
\hline Groundwater & Medium & High & Low & Medium & Low & High & 25 & 50 & 25 \\
\hline Groundwater & Medium & High & Low & Medium & Medium & Low & 50 & 50 & 0 \\
\hline Groundwater & Medium & High & Low & Medium & Medium & Medium & 40 & 55 & 5 \\
\hline Groundwater & Medium & High & Low & Medium & Medium & High & 30 & 55 & 15 \\
\hline Groundwater & Medium & High & Low & Medium & High & Low & 70 & 30 & 0 \\
\hline Groundwater & Medium & High & Low & Medium & High & Medium & 50 & 50 & 0 \\
\hline Groundwater & Medium & High & Low & Medium & High & High & 40 & 60 & 0 \\
\hline Groundwater & Medium & High & Low & High & Low & Low & 45 & 55 & 0 \\
\hline Groundwater & Medium & High & Low & High & Low & Medium & 35 & 45 & 20 \\
\hline Groundwater & Medium & High & Low & High & Low & High & 25 & 45 & 30 \\
\hline Groundwater & Medium & High & Low & High & Medium & Low & 50 & 50 & 0 \\
\hline Groundwater & Medium & High & Low & High & Medium & Medium & 40 & 50 & 10 \\
\hline Groundwater & Medium & High & Low & High & Medium & High & 30 & 50 & 20 \\
\hline Groundwater & Medium & High & Low & High & High & Low & 65 & 35 & 0 \\
\hline Groundwater & Medium & High & Low & High & High & Medium & 50 & 50 & 0 \\
\hline Groundwater & Medium & High & Low & High & High & High & 40 & 60 & 0 \\
\hline Groundwater & Medium & High & Medium & Low & Low & Low & 45 & 55 & 0 \\
\hline Groundwater & Medium & High & Medium & Low & Low & Medium & 35 & 50 & 15 \\
\hline Groundwater & Medium & High & Medium & Low & Low & High & 25 & 50 & 25 \\
\hline Groundwater & Medium & High & Medium & Low & Medium & Low & 50 & 50 & 0 \\
\hline Groundwater & Medium & High & Medium & Low & Medium & Medium & 40 & 55 & 5 \\
\hline Groundwater & Medium & High & Medium & Low & Medium & High & 30 & 55 & 15 \\
\hline Groundwater & Medium & High & Medium & Low & High & Low & 70 & 30 & 0 \\
\hline Groundwater & Medium & High & Medium & Low & High & Medium & 50 & 50 & 0 \\
\hline Groundwater & Medium & High & Medium & Low & High & High & 40 & 60 & 0 \\
\hline Groundwater & Medium & High & Medium & Medium & Low & Low & 40 & 55 & 5 \\
\hline Groundwater & Medium & High & Medium & Medium & Low & Medium & 30 & 45 & 25 \\
\hline Groundwater & Medium & High & Medium & Medium & Low & High & 20 & 45 & 35 \\
\hline Groundwater & Medium & High & Medium & Medium & Medium & Low & 45 & 55 & 0 \\
\hline Groundwater & Medium & High & Medium & Medium & Medium & Medium & 35 & 50 & 15 \\
\hline Groundwater & Medium & High & Medium & Medium & Medium & High & 25 & 50 & 25 \\
\hline Groundwater & Medium & High & Medium & Medium & High & Low & 65 & 35 & 0 \\
\hline Groundwater & Medium & High & Medium & Medium & High & Medium & 45 & 55 & 0 \\
\hline Groundwater & Medium & High & Medium & Medium & High & High & 35 & 60 & 5 \\
\hline Groundwater & Medium & High & Medium & High & Low & Low & 40 & 50 & 10 \\
\hline Groundwater & Medium & High & Medium & High & Low & Medium & 30 & 40 & 30 \\
\hline Groundwater & Medium & High & Medium & High & Low & High & 20 & 40 & 40 \\
\hline Groundwater & Medium & High & Medium & High & Medium & Low & 45 & 55 & 0 \\
\hline Groundwater & Medium & High & Medium & High & Medium & Medium & 35 & 45 & 20 \\
\hline Groundwater & Medium & High & Medium & High & Medium & High & 25 & 45 & 30 \\
\hline
\end{tabular}




\begin{tabular}{|c|c|c|c|c|c|c|c|c|c|}
\hline \multicolumn{7}{|c|}{ Inputs } & \multicolumn{3}{|c|}{ Habitat quality } \\
\hline $\begin{array}{c}\text { Water } \\
\text { availability }\end{array}$ & Emergent & Floating & Terrestrial & $\begin{array}{l}\text { Winter } \\
\text { refuge }\end{array}$ & Submerged & $\begin{array}{l}\text { Water } \\
\text { quality }\end{array}$ & Low & Average & High \\
\hline Groundwater & Medium & High & Medium & High & High & Low & 60 & 40 & 0 \\
\hline Groundwater & Medium & High & Medium & High & High & Medium & 45 & 55 & 0 \\
\hline Groundwater & Medium & High & Medium & High & High & High & 35 & 55 & 10 \\
\hline Groundwater & Medium & High & High & Low & Low & Low & 45 & 55 & 0 \\
\hline Groundwater & Medium & High & High & Low & Low & Medium & 35 & 45 & 20 \\
\hline Groundwater & Medium & High & High & Low & Low & High & 25 & 45 & 30 \\
\hline Groundwater & Medium & High & High & Low & Medium & Low & 50 & 50 & 0 \\
\hline Groundwater & Medium & High & High & Low & Medium & Medium & 40 & 50 & 10 \\
\hline Groundwater & Medium & High & High & Low & Medium & High & 30 & 50 & 20 \\
\hline Groundwater & Medium & High & High & Low & High & Low & 65 & 35 & 0 \\
\hline Groundwater & Medium & High & High & Low & High & Medium & 50 & 50 & 0 \\
\hline Groundwater & Medium & High & High & Low & High & High & 40 & 60 & 0 \\
\hline Groundwater & Medium & High & High & Medium & Low & Low & 40 & 50 & 10 \\
\hline Groundwater & Medium & High & High & Medium & Low & Medium & 30 & 40 & 30 \\
\hline Groundwater & Medium & High & High & Medium & Low & High & 20 & 40 & 40 \\
\hline Groundwater & Medium & High & High & Medium & Medium & Low & 45 & 55 & 0 \\
\hline Groundwater & Medium & High & High & Medium & Medium & Medium & 35 & 45 & 20 \\
\hline Groundwater & Medium & High & High & Medium & Medium & High & 25 & 45 & 30 \\
\hline Groundwater & Medium & High & High & Medium & High & Low & 60 & 40 & 0 \\
\hline Groundwater & Medium & High & High & Medium & High & Medium & 45 & 55 & 0 \\
\hline Groundwater & Medium & High & High & Medium & High & High & 35 & 55 & 10 \\
\hline Groundwater & Medium & High & High & High & Low & Low & 40 & 45 & 15 \\
\hline Groundwater & Medium & High & High & High & Low & Medium & 30 & 35 & 35 \\
\hline Groundwater & Medium & High & High & High & Low & High & 20 & 35 & 45 \\
\hline Groundwater & Medium & High & High & High & Medium & Low & 45 & 50 & 5 \\
\hline Groundwater & Medium & High & High & High & Medium & Medium & 35 & 40 & 25 \\
\hline Groundwater & Medium & High & High & High & Medium & High & 25 & 40 & 35 \\
\hline Groundwater & Medium & High & High & High & High & Low & 55 & 45 & 0 \\
\hline Groundwater & Medium & High & High & High & High & Medium & 45 & 50 & 5 \\
\hline Groundwater & Medium & High & High & High & High & High & 35 & 50 & 15 \\
\hline Groundwater & High & Low & Low & Low & Low & Low & 40 & 60 & 0 \\
\hline Groundwater & High & Low & Low & Low & Low & Medium & 30 & 55 & 15 \\
\hline Groundwater & High & Low & Low & Low & Low & High & 20 & 55 & 25 \\
\hline Groundwater & High & Low & Low & Low & Medium & Low & 45 & 55 & 0 \\
\hline Groundwater & High & Low & Low & Low & Medium & Medium & 35 & 60 & 5 \\
\hline Groundwater & High & Low & Low & Low & Medium & High & 25 & 60 & 15 \\
\hline Groundwater & High & Low & Low & Low & High & Low & 65 & 35 & 0 \\
\hline Groundwater & High & Low & Low & Low & High & Medium & 45 & 55 & 0 \\
\hline Groundwater & High & Low & Low & Low & High & High & 35 & 65 & 0 \\
\hline Groundwater & High & Low & Low & Medium & Low & Low & 35 & 60 & 5 \\
\hline
\end{tabular}




\begin{tabular}{|c|c|c|c|c|c|c|c|c|c|}
\hline \multicolumn{7}{|c|}{ Inputs } & \multicolumn{3}{|c|}{ Habitat quality } \\
\hline $\begin{array}{c}\text { Water } \\
\text { availability }\end{array}$ & Emergent & Floating & Terrestrial & $\begin{array}{l}\text { Winter } \\
\text { refuge }\end{array}$ & Submerged & $\begin{array}{l}\text { Water } \\
\text { quality }\end{array}$ & Low & Average & High \\
\hline Groundwater & High & Low & Low & Medium & Low & Medium & 25 & 50 & 25 \\
\hline Groundwater & High & Low & Low & Medium & Low & High & 15 & 50 & 35 \\
\hline Groundwater & High & Low & Low & Medium & Medium & Low & 40 & 60 & 0 \\
\hline Groundwater & High & Low & Low & Medium & Medium & Medium & 30 & 55 & 15 \\
\hline Groundwater & High & Low & Low & Medium & Medium & High & 20 & 55 & 25 \\
\hline Groundwater & High & Low & Low & Medium & High & Low & 60 & 40 & 0 \\
\hline Groundwater & High & Low & Low & Medium & High & Medium & 40 & 60 & 0 \\
\hline Groundwater & High & Low & Low & Medium & High & High & 30 & 65 & 5 \\
\hline Groundwater & High & Low & Low & High & Low & Low & 35 & 55 & 10 \\
\hline Groundwater & High & Low & Low & High & Low & Medium & 25 & 45 & 30 \\
\hline Groundwater & High & Low & Low & High & Low & High & 15 & 45 & 40 \\
\hline Groundwater & High & Low & Low & High & Medium & Low & 40 & 60 & 0 \\
\hline Groundwater & High & Low & Low & High & Medium & Medium & 30 & 50 & 20 \\
\hline Groundwater & High & Low & Low & High & Medium & High & 20 & 50 & 30 \\
\hline Groundwater & High & Low & Low & High & High & Low & 55 & 45 & 0 \\
\hline Groundwater & High & Low & Low & High & High & Medium & 40 & 60 & 0 \\
\hline Groundwater & High & Low & Low & High & High & High & 30 & 60 & 10 \\
\hline Groundwater & High & Low & Medium & Low & Low & Low & 35 & 60 & 5 \\
\hline Groundwater & High & Low & Medium & Low & Low & Medium & 25 & 50 & 25 \\
\hline Groundwater & High & Low & Medium & Low & Low & High & 15 & 50 & 35 \\
\hline Groundwater & High & Low & Medium & Low & Medium & Low & 40 & 60 & 0 \\
\hline Groundwater & High & Low & Medium & Low & Medium & Medium & 30 & 55 & 15 \\
\hline Groundwater & High & Low & Medium & Low & Medium & High & 20 & 55 & 25 \\
\hline Groundwater & High & Low & Medium & Low & High & Low & 60 & 40 & 0 \\
\hline Groundwater & High & Low & Medium & Low & High & Medium & 40 & 60 & 0 \\
\hline Groundwater & High & Low & Medium & Low & High & High & 30 & 65 & 5 \\
\hline Groundwater & High & Low & Medium & Medium & Low & Low & 30 & 55 & 15 \\
\hline Groundwater & High & Low & Medium & Medium & Low & Medium & 20 & 45 & 35 \\
\hline Groundwater & High & Low & Medium & Medium & Low & High & 10 & 45 & 45 \\
\hline Groundwater & High & Low & Medium & Medium & Medium & Low & 35 & 60 & 5 \\
\hline Groundwater & High & Low & Medium & Medium & Medium & Medium & 25 & 50 & 25 \\
\hline Groundwater & High & Low & Medium & Medium & Medium & High & 15 & 50 & 35 \\
\hline Groundwater & High & Low & Medium & Medium & High & Low & 55 & 45 & 0 \\
\hline Groundwater & High & Low & Medium & Medium & High & Medium & 35 & 60 & 5 \\
\hline Groundwater & High & Low & Medium & Medium & High & High & 25 & 60 & 15 \\
\hline Groundwater & High & Low & Medium & High & Low & Low & 30 & 50 & 20 \\
\hline Groundwater & High & Low & Medium & High & Low & Medium & 20 & 40 & 40 \\
\hline Groundwater & High & Low & Medium & High & Low & High & 10 & 40 & 50 \\
\hline Groundwater & High & Low & Medium & High & Medium & Low & 35 & 55 & 10 \\
\hline Groundwater & High & Low & Medium & High & Medium & Medium & 25 & 45 & 30 \\
\hline
\end{tabular}




\begin{tabular}{|c|c|c|c|c|c|c|c|c|c|}
\hline \multicolumn{7}{|c|}{ Inputs } & \multicolumn{3}{|c|}{ Habitat quality } \\
\hline $\begin{array}{c}\text { Water } \\
\text { availability }\end{array}$ & Emergent & Floating & Terrestrial & $\begin{array}{l}\text { Winter } \\
\text { refuge }\end{array}$ & Submerged & $\begin{array}{l}\text { Water } \\
\text { quality }\end{array}$ & Low & Average & High \\
\hline Groundwater & High & Low & Medium & High & Medium & High & 15 & 45 & 40 \\
\hline Groundwater & High & Low & Medium & High & High & Low & 50 & 50 & 0 \\
\hline Groundwater & High & Low & Medium & High & High & Medium & 35 & 55 & 10 \\
\hline Groundwater & High & Low & Medium & High & High & High & 25 & 55 & 20 \\
\hline Groundwater & High & Low & High & Low & Low & Low & 35 & 55 & 10 \\
\hline Groundwater & High & Low & High & Low & Low & Medium & 25 & 45 & 30 \\
\hline Groundwater & High & Low & High & Low & Low & High & 15 & 45 & 40 \\
\hline Groundwater & High & Low & High & Low & Medium & Low & 40 & 60 & 0 \\
\hline Groundwater & High & Low & High & Low & Medium & Medium & 30 & 50 & 20 \\
\hline Groundwater & High & Low & High & Low & Medium & High & 20 & 50 & 30 \\
\hline Groundwater & High & Low & High & Low & High & Low & 55 & 45 & 0 \\
\hline Groundwater & High & Low & High & Low & High & Medium & 40 & 60 & 0 \\
\hline Groundwater & High & Low & High & Low & High & High & 30 & 60 & 10 \\
\hline Groundwater & High & Low & High & Medium & Low & Low & 30 & 50 & 20 \\
\hline Groundwater & High & Low & High & Medium & Low & Medium & 20 & 40 & 40 \\
\hline Groundwater & High & Low & High & Medium & Low & High & 10 & 40 & 50 \\
\hline Groundwater & High & Low & High & Medium & Medium & Low & 35 & 55 & 10 \\
\hline Groundwater & High & Low & High & Medium & Medium & Medium & 25 & 45 & 30 \\
\hline Groundwater & High & Low & High & Medium & Medium & High & 15 & 45 & 40 \\
\hline Groundwater & High & Low & High & Medium & High & Low & 50 & 50 & 0 \\
\hline Groundwater & High & Low & High & Medium & High & Medium & 35 & 55 & 10 \\
\hline Groundwater & High & Low & High & Medium & High & High & 25 & 55 & 20 \\
\hline Groundwater & High & Low & High & High & Low & Low & 30 & 45 & 25 \\
\hline Groundwater & High & Low & High & High & Low & Medium & 20 & 35 & 45 \\
\hline Groundwater & High & Low & High & High & Low & High & 10 & 35 & 55 \\
\hline Groundwater & High & Low & High & High & Medium & Low & 35 & 50 & 15 \\
\hline Groundwater & High & Low & High & High & Medium & Medium & 25 & 40 & 35 \\
\hline Groundwater & High & Low & High & High & Medium & High & 15 & 40 & 45 \\
\hline Groundwater & High & Low & High & High & High & Low & 45 & 55 & 0 \\
\hline Groundwater & High & Low & High & High & High & Medium & 35 & 50 & 15 \\
\hline Groundwater & High & Low & High & High & High & High & 25 & 50 & 25 \\
\hline Groundwater & High & Medium & Low & Low & Low & Low & 45 & 55 & 0 \\
\hline Groundwater & High & Medium & Low & Low & Low & Medium & 35 & 55 & 10 \\
\hline Groundwater & High & Medium & Low & Low & Low & High & 25 & 55 & 20 \\
\hline Groundwater & High & Medium & Low & Low & Medium & Low & 50 & 50 & 0 \\
\hline Groundwater & High & Medium & Low & Low & Medium & Medium & 40 & 60 & 0 \\
\hline Groundwater & High & Medium & Low & Low & Medium & High & 30 & 60 & 10 \\
\hline Groundwater & High & Medium & Low & Low & High & Low & 70 & 30 & 0 \\
\hline Groundwater & High & Medium & Low & Low & High & Medium & 50 & 50 & 0 \\
\hline Groundwater & High & Medium & Low & Low & High & High & 40 & 60 & 0 \\
\hline
\end{tabular}




\begin{tabular}{|c|c|c|c|c|c|c|c|c|c|}
\hline \multicolumn{7}{|c|}{ Inputs } & \multicolumn{3}{|c|}{ Habitat quality } \\
\hline $\begin{array}{c}\text { Water } \\
\text { availability }\end{array}$ & Emergent & Floating & Terrestrial & $\begin{array}{l}\text { Winter } \\
\text { refuge }\end{array}$ & Submerged & $\begin{array}{l}\text { Water } \\
\text { quality }\end{array}$ & Low & Average & High \\
\hline Groundwater & High & Medium & Low & Medium & Low & Low & 40 & 60 & 0 \\
\hline Groundwater & High & Medium & Low & Medium & Low & Medium & 30 & 50 & 20 \\
\hline Groundwater & High & Medium & Low & Medium & Low & High & 20 & 50 & 30 \\
\hline Groundwater & High & Medium & Low & Medium & Medium & Low & 45 & 55 & 0 \\
\hline Groundwater & High & Medium & Low & Medium & Medium & Medium & 35 & 55 & 10 \\
\hline Groundwater & High & Medium & Low & Medium & Medium & High & 25 & 55 & 20 \\
\hline Groundwater & High & Medium & Low & Medium & High & Low & 65 & 35 & 0 \\
\hline Groundwater & High & Medium & Low & Medium & High & Medium & 45 & 55 & 0 \\
\hline Groundwater & High & Medium & Low & Medium & High & High & 35 & 65 & 0 \\
\hline Groundwater & High & Medium & Low & High & Low & Low & 40 & 55 & 5 \\
\hline Groundwater & High & Medium & Low & High & Low & Medium & 30 & 45 & 25 \\
\hline Groundwater & High & Medium & Low & High & Low & High & 20 & 45 & 35 \\
\hline Groundwater & High & Medium & Low & High & Medium & Low & 45 & 55 & 0 \\
\hline Groundwater & High & Medium & Low & High & Medium & Medium & 35 & 50 & 15 \\
\hline Groundwater & High & Medium & Low & High & Medium & High & 25 & 50 & 25 \\
\hline Groundwater & High & Medium & Low & High & High & Low & 60 & 40 & 0 \\
\hline Groundwater & High & Medium & Low & High & High & Medium & 45 & 55 & 0 \\
\hline Groundwater & High & Medium & Low & High & High & High & 35 & 60 & 5 \\
\hline Groundwater & High & Medium & Medium & Low & Low & Low & 40 & 60 & 0 \\
\hline Groundwater & High & Medium & Medium & Low & Low & Medium & 30 & 50 & 20 \\
\hline Groundwater & High & Medium & Medium & Low & Low & High & 20 & 50 & 30 \\
\hline Groundwater & High & Medium & Medium & Low & Medium & Low & 45 & 55 & 0 \\
\hline Groundwater & High & Medium & Medium & Low & Medium & Medium & 35 & 55 & 10 \\
\hline Groundwater & High & Medium & Medium & Low & Medium & High & 25 & 55 & 20 \\
\hline Groundwater & High & Medium & Medium & Low & High & Low & 65 & 35 & 0 \\
\hline Groundwater & High & Medium & Medium & Low & High & Medium & 45 & 55 & 0 \\
\hline Groundwater & High & Medium & Medium & Low & High & High & 35 & 65 & 0 \\
\hline Groundwater & High & Medium & Medium & Medium & Low & Low & 35 & 55 & 10 \\
\hline Groundwater & High & Medium & Medium & Medium & Low & Medium & 25 & 45 & 30 \\
\hline Groundwater & High & Medium & Medium & Medium & Low & High & 15 & 45 & 40 \\
\hline Groundwater & High & Medium & Medium & Medium & Medium & Low & 40 & 60 & 0 \\
\hline Groundwater & High & Medium & Medium & Medium & Medium & Medium & 30 & 50 & 20 \\
\hline Groundwater & High & Medium & Medium & Medium & Medium & High & 20 & 50 & 30 \\
\hline Groundwater & High & Medium & Medium & Medium & High & Low & 60 & 40 & 0 \\
\hline Groundwater & High & Medium & Medium & Medium & High & Medium & 40 & 60 & 0 \\
\hline Groundwater & High & Medium & Medium & Medium & High & High & 30 & 60 & 10 \\
\hline Groundwater & High & Medium & Medium & High & Low & Low & 35 & 50 & 15 \\
\hline Groundwater & High & Medium & Medium & High & Low & Medium & 25 & 40 & 35 \\
\hline Groundwater & High & Medium & Medium & High & Low & High & 15 & 40 & 45 \\
\hline Groundwater & High & Medium & Medium & High & Medium & Low & 40 & 55 & 5 \\
\hline
\end{tabular}




\begin{tabular}{|c|c|c|c|c|c|c|c|c|c|}
\hline \multicolumn{7}{|c|}{ Inputs } & \multicolumn{3}{|c|}{ Habitat quality } \\
\hline $\begin{array}{c}\text { Water } \\
\text { availability }\end{array}$ & Emergent & Floating & Terrestrial & $\begin{array}{l}\text { Winter } \\
\text { refuge }\end{array}$ & Submerged & $\begin{array}{l}\text { Water } \\
\text { quality }\end{array}$ & Low & Average & High \\
\hline Groundwater & High & Medium & Medium & High & Medium & Medium & 30 & 45 & 25 \\
\hline Groundwater & High & Medium & Medium & High & Medium & High & 20 & 45 & 35 \\
\hline Groundwater & High & Medium & Medium & High & High & Low & 55 & 45 & 0 \\
\hline Groundwater & High & Medium & Medium & High & High & Medium & 40 & 55 & 5 \\
\hline Groundwater & High & Medium & Medium & High & High & High & 30 & 55 & 15 \\
\hline Groundwater & High & Medium & High & Low & Low & Low & 40 & 55 & 5 \\
\hline Groundwater & High & Medium & High & Low & Low & Medium & 30 & 45 & 25 \\
\hline Groundwater & High & Medium & High & Low & Low & High & 20 & 45 & 35 \\
\hline Groundwater & High & Medium & High & Low & Medium & Low & 45 & 55 & 0 \\
\hline Groundwater & High & Medium & High & Low & Medium & Medium & 35 & 50 & 15 \\
\hline Groundwater & High & Medium & High & Low & Medium & High & 25 & 50 & 25 \\
\hline Groundwater & High & Medium & High & Low & High & Low & 60 & 40 & 0 \\
\hline Groundwater & High & Medium & High & Low & High & Medium & 45 & 55 & 0 \\
\hline Groundwater & High & Medium & High & Low & High & High & 35 & 60 & 5 \\
\hline Groundwater & High & Medium & High & Medium & Low & Low & 35 & 50 & 15 \\
\hline Groundwater & High & Medium & High & Medium & Low & Medium & 25 & 40 & 35 \\
\hline Groundwater & High & Medium & High & Medium & Low & High & 15 & 40 & 45 \\
\hline Groundwater & High & Medium & High & Medium & Medium & Low & 40 & 55 & 5 \\
\hline Groundwater & High & Medium & High & Medium & Medium & Medium & 30 & 45 & 25 \\
\hline Groundwater & High & Medium & High & Medium & Medium & High & 20 & 45 & 35 \\
\hline Groundwater & High & Medium & High & Medium & High & Low & 55 & 45 & 0 \\
\hline Groundwater & High & Medium & High & Medium & High & Medium & 40 & 55 & 5 \\
\hline Groundwater & High & Medium & High & Medium & High & High & 30 & 55 & 15 \\
\hline Groundwater & High & Medium & High & High & Low & Low & 35 & 45 & 20 \\
\hline Groundwater & High & Medium & High & High & Low & Medium & 25 & 35 & 40 \\
\hline Groundwater & High & Medium & High & High & Low & High & 15 & 35 & 50 \\
\hline Groundwater & High & Medium & High & High & Medium & Low & 40 & 50 & 10 \\
\hline Groundwater & High & Medium & High & High & Medium & Medium & 30 & 40 & 30 \\
\hline Groundwater & High & Medium & High & High & Medium & High & 20 & 40 & 40 \\
\hline Groundwater & High & Medium & High & High & High & Low & 50 & 50 & 0 \\
\hline Groundwater & High & Medium & High & High & High & Medium & 40 & 50 & 10 \\
\hline Groundwater & High & Medium & High & High & High & High & 30 & 50 & 20 \\
\hline Groundwater & High & High & Low & Low & Low & Low & 50 & 50 & 0 \\
\hline Groundwater & High & High & Low & Low & Low & Medium & 40 & 60 & 0 \\
\hline Groundwater & High & High & Low & Low & Low & High & 30 & 60 & 10 \\
\hline Groundwater & High & High & Low & Low & Medium & Low & 55 & 45 & 0 \\
\hline Groundwater & High & High & Low & Low & Medium & Medium & 45 & 55 & 0 \\
\hline Groundwater & High & High & Low & Low & Medium & High & 35 & 65 & 0 \\
\hline Groundwater & High & High & Low & Low & High & Low & 75 & 25 & 0 \\
\hline Groundwater & High & High & Low & Low & High & Medium & 55 & 45 & 0 \\
\hline
\end{tabular}




\begin{tabular}{|c|c|c|c|c|c|c|c|c|c|}
\hline \multicolumn{7}{|c|}{ Inputs } & \multicolumn{3}{|c|}{ Habitat quality } \\
\hline $\begin{array}{c}\text { Water } \\
\text { availability }\end{array}$ & Emergent & Floating & Terrestrial & $\begin{array}{l}\text { Winter } \\
\text { refuge }\end{array}$ & Submerged & $\begin{array}{l}\text { Water } \\
\text { quality }\end{array}$ & Low & Average & High \\
\hline Groundwater & High & High & Low & Low & High & High & 45 & 55 & 0 \\
\hline Groundwater & High & High & Low & Medium & Low & Low & 45 & 55 & 0 \\
\hline Groundwater & High & High & Low & Medium & Low & Medium & 35 & 55 & 10 \\
\hline Groundwater & High & High & Low & Medium & Low & High & 25 & 55 & 20 \\
\hline Groundwater & High & High & Low & Medium & Medium & Low & 50 & 50 & 0 \\
\hline Groundwater & High & High & Low & Medium & Medium & Medium & 40 & 60 & 0 \\
\hline Groundwater & High & High & Low & Medium & Medium & High & 30 & 60 & 10 \\
\hline Groundwater & High & High & Low & Medium & High & Low & 70 & 30 & 0 \\
\hline Groundwater & High & High & Low & Medium & High & Medium & 50 & 50 & 0 \\
\hline Groundwater & High & High & Low & Medium & High & High & 40 & 60 & 0 \\
\hline Groundwater & High & High & Low & High & Low & Low & 45 & 55 & 0 \\
\hline Groundwater & High & High & Low & High & Low & Medium & 35 & 50 & 15 \\
\hline Groundwater & High & High & Low & High & Low & High & 25 & 50 & 25 \\
\hline Groundwater & High & High & Low & High & Medium & Low & 50 & 50 & 0 \\
\hline Groundwater & High & High & Low & High & Medium & Medium & 40 & 55 & 5 \\
\hline Groundwater & High & High & Low & High & Medium & High & 30 & 55 & 15 \\
\hline Groundwater & High & High & Low & High & High & Low & 65 & 35 & 0 \\
\hline Groundwater & High & High & Low & High & High & Medium & 50 & 50 & 0 \\
\hline Groundwater & High & High & Low & High & High & High & 40 & 60 & 0 \\
\hline Groundwater & High & High & Medium & Low & Low & Low & 45 & 55 & 0 \\
\hline Groundwater & High & High & Medium & Low & Low & Medium & 35 & 55 & 10 \\
\hline Groundwater & High & High & Medium & Low & Low & High & 25 & 55 & 20 \\
\hline Groundwater & High & High & Medium & Low & Medium & Low & 50 & 50 & 0 \\
\hline Groundwater & High & High & Medium & Low & Medium & Medium & 40 & 60 & 0 \\
\hline Groundwater & High & High & Medium & Low & Medium & High & 30 & 60 & 10 \\
\hline Groundwater & High & High & Medium & Low & High & Low & 70 & 30 & 0 \\
\hline Groundwater & High & High & Medium & Low & High & Medium & 50 & 50 & 0 \\
\hline Groundwater & High & High & Medium & Low & High & High & 40 & 60 & 0 \\
\hline Groundwater & High & High & Medium & Medium & Low & Low & 40 & 60 & 0 \\
\hline Groundwater & High & High & Medium & Medium & Low & Medium & 30 & 50 & 20 \\
\hline Groundwater & High & High & Medium & Medium & Low & High & 20 & 50 & 30 \\
\hline Groundwater & High & High & Medium & Medium & Medium & Low & 45 & 55 & 0 \\
\hline Groundwater & High & High & Medium & Medium & Medium & Medium & 35 & 55 & 10 \\
\hline Groundwater & High & High & Medium & Medium & Medium & High & 25 & 55 & 20 \\
\hline Groundwater & High & High & Medium & Medium & High & Low & 65 & 35 & 0 \\
\hline Groundwater & High & High & Medium & Medium & High & Medium & 45 & 55 & 0 \\
\hline Groundwater & High & High & Medium & Medium & High & High & 35 & 65 & 0 \\
\hline Groundwater & High & High & Medium & High & Low & Low & 40 & 55 & 5 \\
\hline Groundwater & High & High & Medium & High & Low & Medium & 30 & 45 & 25 \\
\hline Groundwater & High & High & Medium & High & Low & High & 20 & 45 & 35 \\
\hline
\end{tabular}




\begin{tabular}{|c|c|c|c|c|c|c|c|c|c|}
\hline \multicolumn{7}{|c|}{ Inputs } & \multicolumn{3}{|c|}{ Habitat quality } \\
\hline $\begin{array}{c}\text { Water } \\
\text { availability }\end{array}$ & Emergent & Floating & Terrestrial & $\begin{array}{l}\text { Winter } \\
\text { refuge }\end{array}$ & Submerged & $\begin{array}{l}\text { Water } \\
\text { quality }\end{array}$ & Low & Average & High \\
\hline Groundwater & High & High & Medium & High & Medium & Low & 45 & 55 & 0 \\
\hline Groundwater & High & High & Medium & High & Medium & Medium & 35 & 50 & 15 \\
\hline Groundwater & High & High & Medium & High & Medium & High & 25 & 50 & 25 \\
\hline Groundwater & High & High & Medium & High & High & Low & 60 & 40 & 0 \\
\hline Groundwater & High & High & Medium & High & High & Medium & 45 & 55 & 0 \\
\hline Groundwater & High & High & Medium & High & High & High & 35 & 60 & 5 \\
\hline Groundwater & High & High & High & Low & Low & Low & 45 & 55 & 0 \\
\hline Groundwater & High & High & High & Low & Low & Medium & 35 & 50 & 15 \\
\hline Groundwater & High & High & High & Low & Low & High & 25 & 50 & 25 \\
\hline Groundwater & High & High & High & Low & Medium & Low & 50 & 50 & 0 \\
\hline Groundwater & High & High & High & Low & Medium & Medium & 40 & 55 & 5 \\
\hline Groundwater & High & High & High & Low & Medium & High & 30 & 55 & 15 \\
\hline Groundwater & High & High & High & Low & High & Low & 65 & 35 & 0 \\
\hline Groundwater & High & High & High & Low & High & Medium & 50 & 50 & 0 \\
\hline Groundwater & High & High & High & Low & High & High & 40 & 60 & 0 \\
\hline Groundwater & High & High & High & Medium & Low & Low & 40 & 55 & 5 \\
\hline Groundwater & High & High & High & Medium & Low & Medium & 30 & 45 & 25 \\
\hline Groundwater & High & High & High & Medium & Low & High & 20 & 45 & 35 \\
\hline Groundwater & High & High & High & Medium & Medium & Low & 45 & 55 & 0 \\
\hline Groundwater & High & High & High & Medium & Medium & Medium & 35 & 50 & 15 \\
\hline Groundwater & High & High & High & Medium & Medium & High & 25 & 50 & 25 \\
\hline Groundwater & High & High & High & Medium & High & Low & 60 & 40 & 0 \\
\hline Groundwater & High & High & High & Medium & High & Medium & 45 & 55 & 0 \\
\hline Groundwater & High & High & High & Medium & High & High & 35 & 60 & 5 \\
\hline Groundwater & High & High & High & High & Low & Low & 40 & 50 & 10 \\
\hline Groundwater & High & High & High & High & Low & Medium & 30 & 40 & 30 \\
\hline Groundwater & High & High & High & High & Low & High & 20 & 40 & 40 \\
\hline Groundwater & High & High & High & High & Medium & Low & 45 & 55 & 0 \\
\hline Groundwater & High & High & High & High & Medium & Medium & 35 & 45 & 20 \\
\hline Groundwater & High & High & High & High & Medium & High & 25 & 45 & 30 \\
\hline Groundwater & High & High & High & High & High & Low & 55 & 45 & 0 \\
\hline Groundwater & High & High & High & High & High & Medium & 45 & 55 & 0 \\
\hline Groundwater & High & High & High & High & High & High & 35 & 55 & 10 \\
\hline Summer & Low & Low & Low & Low & Low & Low & 35 & 55 & 10 \\
\hline Summer & Low & Low & Low & Low & Low & Medium & 25 & 45 & 30 \\
\hline Summer & Low & Low & Low & Low & Low & High & 15 & 45 & 40 \\
\hline Summer & Low & Low & Low & Low & Medium & Low & 40 & 60 & 0 \\
\hline Summer & Low & Low & Low & Low & Medium & Medium & 30 & 50 & 20 \\
\hline Summer & Low & Low & Low & Low & Medium & High & 20 & 50 & 30 \\
\hline Summer & Low & Low & Low & Low & High & Low & 45 & 55 & 0 \\
\hline
\end{tabular}




\begin{tabular}{|c|c|c|c|c|c|c|c|c|c|}
\hline \multicolumn{7}{|c|}{ Inputs } & \multicolumn{3}{|c|}{ Habitat quality } \\
\hline $\begin{array}{c}\text { Water } \\
\text { availability }\end{array}$ & Emergent & Floating & Terrestrial & $\begin{array}{l}\text { Winter } \\
\text { refuge }\end{array}$ & Submerged & $\begin{array}{l}\text { Water } \\
\text { quality }\end{array}$ & Low & Average & High \\
\hline Summer & Low & Low & Low & Low & High & Medium & 40 & 60 & 0 \\
\hline Summer & Low & Low & Low & Low & High & High & 30 & 60 & 10 \\
\hline Summer & Low & Low & Low & Medium & Low & Low & 30 & 50 & 20 \\
\hline Summer & Low & Low & Low & Medium & Low & Medium & 20 & 40 & 40 \\
\hline Summer & Low & Low & Low & Medium & Low & High & 10 & 40 & 50 \\
\hline Summer & Low & Low & Low & Medium & Medium & Low & 35 & 55 & 10 \\
\hline Summer & Low & Low & Low & Medium & Medium & Medium & 25 & 45 & 30 \\
\hline Summer & Low & Low & Low & Medium & Medium & High & 15 & 45 & 40 \\
\hline Summer & Low & Low & Low & Medium & High & Low & 40 & 60 & 0 \\
\hline Summer & Low & Low & Low & Medium & High & Medium & 35 & 55 & 10 \\
\hline Summer & Low & Low & Low & Medium & High & High & 25 & 55 & 20 \\
\hline Summer & Low & Low & Low & High & Low & Low & 30 & 45 & 25 \\
\hline Summer & Low & Low & Low & High & Low & Medium & 20 & 35 & 45 \\
\hline Summer & Low & Low & Low & High & Low & High & 10 & 35 & 55 \\
\hline Summer & Low & Low & Low & High & Medium & Low & 35 & 50 & 15 \\
\hline Summer & Low & Low & Low & High & Medium & Medium & 25 & 40 & 35 \\
\hline Summer & Low & Low & Low & High & Medium & High & 15 & 40 & 45 \\
\hline Summer & Low & Low & Low & High & High & Low & 45 & 55 & 0 \\
\hline Summer & Low & Low & Low & High & High & Medium & 35 & 50 & 15 \\
\hline Summer & Low & Low & Low & High & High & High & 25 & 50 & 25 \\
\hline Summer & Low & Low & Medium & Low & Low & Low & 30 & 50 & 20 \\
\hline Summer & Low & Low & Medium & Low & Low & Medium & 20 & 40 & 40 \\
\hline Summer & Low & Low & Medium & Low & Low & High & 10 & 40 & 50 \\
\hline Summer & Low & Low & Medium & Low & Medium & Low & 35 & 55 & 10 \\
\hline Summer & Low & Low & Medium & Low & Medium & Medium & 25 & 45 & 30 \\
\hline Summer & Low & Low & Medium & Low & Medium & High & 15 & 45 & 40 \\
\hline Summer & Low & Low & Medium & Low & High & Low & 40 & 60 & 0 \\
\hline Summer & Low & Low & Medium & Low & High & Medium & 35 & 55 & 10 \\
\hline Summer & Low & Low & Medium & Low & High & High & 25 & 55 & 20 \\
\hline Summer & Low & Low & Medium & Medium & Low & Low & 25 & 45 & 30 \\
\hline Summer & Low & Low & Medium & Medium & Low & Medium & 15 & 35 & 50 \\
\hline Summer & Low & Low & Medium & Medium & Low & High & 5 & 35 & 60 \\
\hline Summer & Low & Low & Medium & Medium & Medium & Low & 30 & 50 & 20 \\
\hline Summer & Low & Low & Medium & Medium & Medium & Medium & 20 & 40 & 40 \\
\hline Summer & Low & Low & Medium & Medium & Medium & High & 10 & 40 & 50 \\
\hline Summer & Low & Low & Medium & Medium & High & Low & 40 & 60 & 0 \\
\hline Summer & Low & Low & Medium & Medium & High & Medium & 30 & 50 & 20 \\
\hline Summer & Low & Low & Medium & Medium & High & High & 20 & 50 & 30 \\
\hline Summer & Low & Low & Medium & High & Low & Low & 25 & 40 & 35 \\
\hline Summer & Low & Low & Medium & High & Low & Medium & 15 & 30 & 55 \\
\hline
\end{tabular}




\begin{tabular}{|c|c|c|c|c|c|c|c|c|c|}
\hline \multicolumn{7}{|c|}{ Inputs } & \multicolumn{3}{|c|}{ Habitat quality } \\
\hline $\begin{array}{c}\text { Water } \\
\text { availability }\end{array}$ & Emergent & Floating & Terrestrial & $\begin{array}{l}\text { Winter } \\
\text { refuge }\end{array}$ & Submerged & $\begin{array}{l}\text { Water } \\
\text { quality }\end{array}$ & Low & Average & High \\
\hline Summer & Low & Low & Medium & High & Low & High & 5 & 30 & 65 \\
\hline Summer & Low & Low & Medium & High & Medium & Low & 30 & 45 & 25 \\
\hline Summer & Low & Low & Medium & High & Medium & Medium & 20 & 35 & 45 \\
\hline Summer & Low & Low & Medium & High & Medium & High & 10 & 35 & 55 \\
\hline Summer & Low & Low & Medium & High & High & Low & 40 & 55 & 5 \\
\hline Summer & Low & Low & Medium & High & High & Medium & 30 & 45 & 25 \\
\hline Summer & Low & Low & Medium & High & High & High & 20 & 45 & 35 \\
\hline Summer & Low & Low & High & Low & Low & Low & 30 & 45 & 25 \\
\hline Summer & Low & Low & High & Low & Low & Medium & 20 & 35 & 45 \\
\hline Summer & Low & Low & High & Low & Low & High & 10 & 35 & 55 \\
\hline Summer & Low & Low & High & Low & Medium & Low & 35 & 50 & 15 \\
\hline Summer & Low & Low & High & Low & Medium & Medium & 25 & 40 & 35 \\
\hline Summer & Low & Low & High & Low & Medium & High & 15 & 40 & 45 \\
\hline Summer & Low & Low & High & Low & High & Low & 45 & 55 & 0 \\
\hline Summer & Low & Low & High & Low & High & Medium & 35 & 50 & 15 \\
\hline Summer & Low & Low & High & Low & High & High & 25 & 50 & 25 \\
\hline Summer & Low & Low & High & Medium & Low & Low & 25 & 40 & 35 \\
\hline Summer & Low & Low & High & Medium & Low & Medium & 15 & 30 & 55 \\
\hline Summer & Low & Low & High & Medium & Low & High & 5 & 30 & 65 \\
\hline Summer & Low & Low & High & Medium & Medium & Low & 30 & 45 & 25 \\
\hline Summer & Low & Low & High & Medium & Medium & Medium & 20 & 35 & 45 \\
\hline Summer & Low & Low & High & Medium & Medium & High & 10 & 35 & 55 \\
\hline Summer & Low & Low & High & Medium & High & Low & 40 & 55 & 5 \\
\hline Summer & Low & Low & High & Medium & High & Medium & 30 & 45 & 25 \\
\hline Summer & Low & Low & High & Medium & High & High & 20 & 45 & 35 \\
\hline Summer & Low & Low & High & High & Low & Low & 25 & 35 & 40 \\
\hline Summer & Low & Low & High & High & Low & Medium & 15 & 25 & 60 \\
\hline Summer & Low & Low & High & High & Low & High & 5 & 25 & 70 \\
\hline Summer & Low & Low & High & High & Medium & Low & 30 & 40 & 30 \\
\hline Summer & Low & Low & High & High & Medium & Medium & 20 & 30 & 50 \\
\hline Summer & Low & Low & High & High & Medium & High & 10 & 30 & 60 \\
\hline Summer & Low & Low & High & High & High & Low & 40 & 50 & 10 \\
\hline Summer & Low & Low & High & High & High & Medium & 30 & 40 & 30 \\
\hline Summer & Low & Low & High & High & High & High & 20 & 40 & 40 \\
\hline Summer & Low & Medium & Low & Low & Low & Low & 40 & 55 & 5 \\
\hline Summer & Low & Medium & Low & Low & Low & Medium & 30 & 45 & 25 \\
\hline Summer & Low & Medium & Low & Low & Low & High & 20 & 45 & 35 \\
\hline Summer & Low & Medium & Low & Low & Medium & Low & 45 & 55 & 0 \\
\hline Summer & Low & Medium & Low & Low & Medium & Medium & 35 & 50 & 15 \\
\hline Summer & Low & Medium & Low & Low & Medium & High & 25 & 50 & 25 \\
\hline
\end{tabular}




\begin{tabular}{|c|c|c|c|c|c|c|c|c|c|}
\hline \multicolumn{7}{|c|}{ Inputs } & \multicolumn{3}{|c|}{ Habitat quality } \\
\hline $\begin{array}{c}\text { Water } \\
\text { availability }\end{array}$ & Emergent & Floating & Terrestrial & $\begin{array}{l}\text { Winter } \\
\text { refuge }\end{array}$ & Submerged & $\begin{array}{l}\text { Water } \\
\text { quality }\end{array}$ & Low & Average & High \\
\hline Summer & Low & Medium & Low & Low & High & Low & 50 & 50 & 0 \\
\hline Summer & Low & Medium & Low & Low & High & Medium & 45 & 55 & 0 \\
\hline Summer & Low & Medium & Low & Low & High & High & 35 & 60 & 5 \\
\hline Summer & Low & Medium & Low & Medium & Low & Low & 35 & 50 & 15 \\
\hline Summer & Low & Medium & Low & Medium & Low & Medium & 25 & 40 & 35 \\
\hline Summer & Low & Medium & Low & Medium & Low & High & 15 & 40 & 45 \\
\hline Summer & Low & Medium & Low & Medium & Medium & Low & 40 & 55 & 5 \\
\hline Summer & Low & Medium & Low & Medium & Medium & Medium & 30 & 45 & 25 \\
\hline Summer & Low & Medium & Low & Medium & Medium & High & 20 & 45 & 35 \\
\hline Summer & Low & Medium & Low & Medium & High & Low & 45 & 55 & 0 \\
\hline Summer & Low & Medium & Low & Medium & High & Medium & 40 & 55 & 5 \\
\hline Summer & Low & Medium & Low & Medium & High & High & 30 & 55 & 15 \\
\hline Summer & Low & Medium & Low & High & Low & Low & 35 & 45 & 20 \\
\hline Summer & Low & Medium & Low & High & Low & Medium & 25 & 35 & 40 \\
\hline Summer & Low & Medium & Low & High & Low & High & 15 & 35 & 50 \\
\hline Summer & Low & Medium & Low & High & Medium & Low & 40 & 50 & 10 \\
\hline Summer & Low & Medium & Low & High & Medium & Medium & 30 & 40 & 30 \\
\hline Summer & Low & Medium & Low & High & Medium & High & 20 & 40 & 40 \\
\hline Summer & Low & Medium & Low & High & High & Low & 45 & 55 & 0 \\
\hline Summer & Low & Medium & Low & High & High & Medium & 40 & 50 & 10 \\
\hline Summer & Low & Medium & Low & High & High & High & 30 & 50 & 20 \\
\hline Summer & Low & Medium & Medium & Low & Low & Low & 35 & 50 & 15 \\
\hline Summer & Low & Medium & Medium & Low & Low & Medium & 25 & 40 & 35 \\
\hline Summer & Low & Medium & Medium & Low & Low & High & 15 & 40 & 45 \\
\hline Summer & Low & Medium & Medium & Low & Medium & Low & 40 & 55 & 5 \\
\hline Summer & Low & Medium & Medium & Low & Medium & Medium & 30 & 45 & 25 \\
\hline Summer & Low & Medium & Medium & Low & Medium & High & 20 & 45 & 35 \\
\hline Summer & Low & Medium & Medium & Low & High & Low & 45 & 55 & 0 \\
\hline Summer & Low & Medium & Medium & Low & High & Medium & 40 & 55 & 5 \\
\hline Summer & Low & Medium & Medium & Low & High & High & 30 & 55 & 15 \\
\hline Summer & Low & Medium & Medium & Medium & Low & Low & 30 & 45 & 25 \\
\hline Summer & Low & Medium & Medium & Medium & Low & Medium & 20 & 35 & 45 \\
\hline Summer & Low & Medium & Medium & Medium & Low & High & 10 & 35 & 55 \\
\hline Summer & Low & Medium & Medium & Medium & Medium & Low & 35 & 50 & 15 \\
\hline Summer & Low & Medium & Medium & Medium & Medium & Medium & 25 & 40 & 35 \\
\hline Summer & Low & Medium & Medium & Medium & Medium & High & 15 & 40 & 45 \\
\hline Summer & Low & Medium & Medium & Medium & High & Low & 45 & 55 & 0 \\
\hline Summer & Low & Medium & Medium & Medium & High & Medium & 35 & 50 & 15 \\
\hline Summer & Low & Medium & Medium & Medium & High & High & 25 & 50 & 25 \\
\hline Summer & Low & Medium & Medium & High & Low & Low & 30 & 40 & 30 \\
\hline
\end{tabular}




\begin{tabular}{|c|c|c|c|c|c|c|c|c|c|}
\hline \multicolumn{7}{|c|}{ Inputs } & \multicolumn{3}{|c|}{ Habitat quality } \\
\hline $\begin{array}{c}\text { Water } \\
\text { availability }\end{array}$ & Emergent & Floating & Terrestrial & $\begin{array}{l}\text { Winter } \\
\text { refuge }\end{array}$ & Submerged & $\begin{array}{l}\text { Water } \\
\text { quality }\end{array}$ & Low & Average & High \\
\hline Summer & Low & Medium & Medium & High & Low & Medium & 20 & 30 & 50 \\
\hline Summer & Low & Medium & Medium & High & Low & High & 10 & 30 & 60 \\
\hline Summer & Low & Medium & Medium & High & Medium & Low & 35 & 45 & 20 \\
\hline Summer & Low & Medium & Medium & High & Medium & Medium & 25 & 35 & 40 \\
\hline Summer & Low & Medium & Medium & High & Medium & High & 15 & 35 & 50 \\
\hline Summer & Low & Medium & Medium & High & High & Low & 45 & 55 & 0 \\
\hline Summer & Low & Medium & Medium & High & High & Medium & 35 & 45 & 20 \\
\hline Summer & Low & Medium & Medium & High & High & High & 25 & 45 & 30 \\
\hline Summer & Low & Medium & High & Low & Low & Low & 35 & 45 & 20 \\
\hline Summer & Low & Medium & High & Low & Low & Medium & 25 & 35 & 40 \\
\hline Summer & Low & Medium & High & Low & Low & High & 15 & 35 & 50 \\
\hline Summer & Low & Medium & High & Low & Medium & Low & 40 & 50 & 10 \\
\hline Summer & Low & Medium & High & Low & Medium & Medium & 30 & 40 & 30 \\
\hline Summer & Low & Medium & High & Low & Medium & High & 20 & 40 & 40 \\
\hline Summer & Low & Medium & High & Low & High & Low & 45 & 55 & 0 \\
\hline Summer & Low & Medium & High & Low & High & Medium & 40 & 50 & 10 \\
\hline Summer & Low & Medium & High & Low & High & High & 30 & 50 & 20 \\
\hline Summer & Low & Medium & High & Medium & Low & Low & 30 & 40 & 30 \\
\hline Summer & Low & Medium & High & Medium & Low & Medium & 20 & 30 & 50 \\
\hline Summer & Low & Medium & High & Medium & Low & High & 10 & 30 & 60 \\
\hline Summer & Low & Medium & High & Medium & Medium & Low & 35 & 45 & 20 \\
\hline Summer & Low & Medium & High & Medium & Medium & Medium & 25 & 35 & 40 \\
\hline Summer & Low & Medium & High & Medium & Medium & High & 15 & 35 & 50 \\
\hline Summer & Low & Medium & High & Medium & High & Low & 45 & 55 & 0 \\
\hline Summer & Low & Medium & High & Medium & High & Medium & 35 & 45 & 20 \\
\hline Summer & Low & Medium & High & Medium & High & High & 25 & 45 & 30 \\
\hline Summer & Low & Medium & High & High & Low & Low & 30 & 35 & 35 \\
\hline Summer & Low & Medium & High & High & Low & Medium & 20 & 25 & 55 \\
\hline Summer & Low & Medium & High & High & Low & High & 10 & 25 & 65 \\
\hline Summer & Low & Medium & High & High & Medium & Low & 35 & 40 & 25 \\
\hline Summer & Low & Medium & High & High & Medium & Medium & 25 & 30 & 45 \\
\hline Summer & Low & Medium & High & High & Medium & High & 15 & 30 & 55 \\
\hline Summer & Low & Medium & High & High & High & Low & 45 & 50 & 5 \\
\hline Summer & Low & Medium & High & High & High & Medium & 35 & 40 & 25 \\
\hline Summer & Low & Medium & High & High & High & High & 25 & 40 & 35 \\
\hline Summer & Low & High & Low & Low & Low & Low & 45 & 55 & 0 \\
\hline Summer & Low & High & Low & Low & Low & Medium & 35 & 50 & 15 \\
\hline Summer & Low & High & Low & Low & Low & High & 25 & 50 & 25 \\
\hline Summer & Low & High & Low & Low & Medium & Low & 45 & 55 & 0 \\
\hline Summer & Low & High & Low & Low & Medium & Medium & 40 & 55 & 5 \\
\hline
\end{tabular}




\begin{tabular}{|c|c|c|c|c|c|c|c|c|c|}
\hline \multicolumn{7}{|c|}{ Inputs } & \multicolumn{3}{|c|}{ Habitat quality } \\
\hline $\begin{array}{c}\text { Water } \\
\text { availability }\end{array}$ & Emergent & Floating & Terrestrial & $\begin{array}{l}\text { Winter } \\
\text { refuge }\end{array}$ & Submerged & $\begin{array}{l}\text { Water } \\
\text { quality }\end{array}$ & Low & Average & High \\
\hline Summer & Low & High & Low & Low & Medium & High & 30 & 55 & 15 \\
\hline Summer & Low & High & Low & Low & High & Low & 55 & 45 & 0 \\
\hline Summer & Low & High & Low & Low & High & Medium & 45 & 55 & 0 \\
\hline Summer & Low & High & Low & Low & High & High & 40 & 60 & 0 \\
\hline Summer & Low & High & Low & Medium & Low & Low & 40 & 55 & 5 \\
\hline Summer & Low & High & Low & Medium & Low & Medium & 30 & 45 & 25 \\
\hline Summer & Low & High & Low & Medium & Low & High & 20 & 45 & 35 \\
\hline Summer & Low & High & Low & Medium & Medium & Low & 45 & 55 & 0 \\
\hline Summer & Low & High & Low & Medium & Medium & Medium & 35 & 50 & 15 \\
\hline Summer & Low & High & Low & Medium & Medium & High & 25 & 50 & 25 \\
\hline Summer & Low & High & Low & Medium & High & Low & 45 & 55 & 0 \\
\hline Summer & Low & High & Low & Medium & High & Medium & 45 & 55 & 0 \\
\hline Summer & Low & High & Low & Medium & High & High & 35 & 60 & 5 \\
\hline Summer & Low & High & Low & High & Low & Low & 40 & 50 & 10 \\
\hline Summer & Low & High & Low & High & Low & Medium & 30 & 40 & 30 \\
\hline Summer & Low & High & Low & High & Low & High & 20 & 40 & 40 \\
\hline Summer & Low & High & Low & High & Medium & Low & 45 & 55 & 0 \\
\hline Summer & Low & High & Low & High & Medium & Medium & 35 & 45 & 20 \\
\hline Summer & Low & High & Low & High & Medium & High & 25 & 45 & 30 \\
\hline Summer & Low & High & Low & High & High & Low & 45 & 55 & 0 \\
\hline Summer & Low & High & Low & High & High & Medium & 45 & 55 & 0 \\
\hline Summer & Low & High & Low & High & High & High & 35 & 55 & 10 \\
\hline Summer & Low & High & Medium & Low & Low & Low & 40 & 55 & 5 \\
\hline Summer & Low & High & Medium & Low & Low & Medium & 30 & 45 & 25 \\
\hline Summer & Low & High & Medium & Low & Low & High & 20 & 45 & 35 \\
\hline Summer & Low & High & Medium & Low & Medium & Low & 45 & 55 & 0 \\
\hline Summer & Low & High & Medium & Low & Medium & Medium & 35 & 50 & 15 \\
\hline Summer & Low & High & Medium & Low & Medium & High & 25 & 50 & 25 \\
\hline Summer & Low & High & Medium & Low & High & Low & 45 & 55 & 0 \\
\hline Summer & Low & High & Medium & Low & High & Medium & 45 & 55 & 0 \\
\hline Summer & Low & High & Medium & Low & High & High & 35 & 60 & 5 \\
\hline Summer & Low & High & Medium & Medium & Low & Low & 35 & 50 & 15 \\
\hline Summer & Low & High & Medium & Medium & Low & Medium & 25 & 40 & 35 \\
\hline Summer & Low & High & Medium & Medium & Low & High & 15 & 40 & 45 \\
\hline Summer & Low & High & Medium & Medium & Medium & Low & 40 & 55 & 5 \\
\hline Summer & Low & High & Medium & Medium & Medium & Medium & 30 & 45 & 25 \\
\hline Summer & Low & High & Medium & Medium & Medium & High & 20 & 45 & 35 \\
\hline Summer & Low & High & Medium & Medium & High & Low & 45 & 55 & 0 \\
\hline Summer & Low & High & Medium & Medium & High & Medium & 40 & 55 & 5 \\
\hline Summer & Low & High & Medium & Medium & High & High & 30 & 55 & 15 \\
\hline
\end{tabular}




\begin{tabular}{|c|c|c|c|c|c|c|c|c|c|}
\hline \multicolumn{7}{|c|}{ Inputs } & \multicolumn{3}{|c|}{ Habitat quality } \\
\hline $\begin{array}{c}\text { Water } \\
\text { availability }\end{array}$ & Emergent & Floating & Terrestrial & $\begin{array}{l}\text { Winter } \\
\text { refuge }\end{array}$ & Submerged & $\begin{array}{l}\text { Water } \\
\text { quality }\end{array}$ & Low & Average & High \\
\hline Summer & Low & High & Medium & High & Low & Low & 35 & 45 & 20 \\
\hline Summer & Low & High & Medium & High & Low & Medium & 25 & 35 & 40 \\
\hline Summer & Low & High & Medium & High & Low & High & 15 & 35 & 50 \\
\hline Summer & Low & High & Medium & High & Medium & Low & 40 & 50 & 10 \\
\hline Summer & Low & High & Medium & High & Medium & Medium & 30 & 40 & 30 \\
\hline Summer & Low & High & Medium & High & Medium & High & 20 & 40 & 40 \\
\hline Summer & Low & High & Medium & High & High & Low & 45 & 55 & 0 \\
\hline Summer & Low & High & Medium & High & High & Medium & 40 & 50 & 10 \\
\hline Summer & Low & High & Medium & High & High & High & 30 & 50 & 20 \\
\hline Summer & Low & High & High & Low & Low & Low & 40 & 50 & 10 \\
\hline Summer & Low & High & High & Low & Low & Medium & 30 & 40 & 30 \\
\hline Summer & Low & High & High & Low & Low & High & 20 & 40 & 40 \\
\hline Summer & Low & High & High & Low & Medium & Low & 45 & 55 & 0 \\
\hline Summer & Low & High & High & Low & Medium & Medium & 35 & 45 & 20 \\
\hline Summer & Low & High & High & Low & Medium & High & 25 & 45 & 30 \\
\hline Summer & Low & High & High & Low & High & Low & 45 & 55 & 0 \\
\hline Summer & Low & High & High & Low & High & Medium & 45 & 55 & 0 \\
\hline Summer & Low & High & High & Low & High & High & 35 & 55 & 10 \\
\hline Summer & Low & High & High & Medium & Low & Low & 35 & 45 & 20 \\
\hline Summer & Low & High & High & Medium & Low & Medium & 25 & 35 & 40 \\
\hline Summer & Low & High & High & Medium & Low & High & 15 & 35 & 50 \\
\hline Summer & Low & High & High & Medium & Medium & Low & 40 & 50 & 10 \\
\hline Summer & Low & High & High & Medium & Medium & Medium & 30 & 40 & 30 \\
\hline Summer & Low & High & High & Medium & Medium & High & 20 & 40 & 40 \\
\hline Summer & Low & High & High & Medium & High & Low & 45 & 55 & 0 \\
\hline Summer & Low & High & High & Medium & High & Medium & 40 & 50 & 10 \\
\hline Summer & Low & High & High & Medium & High & High & 30 & 50 & 20 \\
\hline Summer & Low & High & High & High & Low & Low & 35 & 40 & 25 \\
\hline Summer & Low & High & High & High & Low & Medium & 25 & 30 & 45 \\
\hline Summer & Low & High & High & High & Low & High & 15 & 30 & 55 \\
\hline Summer & Low & High & High & High & Medium & Low & 40 & 45 & 15 \\
\hline Summer & Low & High & High & High & Medium & Medium & 30 & 35 & 35 \\
\hline Summer & Low & High & High & High & Medium & High & 20 & 35 & 45 \\
\hline Summer & Low & High & High & High & High & Low & 50 & 50 & 0 \\
\hline Summer & Low & High & High & High & High & Medium & 40 & 45 & 15 \\
\hline Summer & Low & High & High & High & High & High & 30 & 45 & 25 \\
\hline Summer & Medium & Low & Low & Low & Low & Low & 30 & 50 & 20 \\
\hline Summer & Medium & Low & Low & Low & Low & Medium & 20 & 40 & 40 \\
\hline Summer & Medium & Low & Low & Low & Low & High & 10 & 40 & 50 \\
\hline Summer & Medium & Low & Low & Low & Medium & Low & 35 & 55 & 10 \\
\hline
\end{tabular}




\begin{tabular}{|c|c|c|c|c|c|c|c|c|c|}
\hline \multicolumn{7}{|c|}{ Inputs } & \multicolumn{3}{|c|}{ Habitat quality } \\
\hline $\begin{array}{c}\text { Water } \\
\text { availability }\end{array}$ & Emergent & Floating & Terrestrial & $\begin{array}{l}\text { Winter } \\
\text { refuge }\end{array}$ & Submerged & $\begin{array}{l}\text { Water } \\
\text { quality }\end{array}$ & Low & Average & High \\
\hline Summer & Medium & Low & Low & Low & Medium & Medium & 25 & 45 & 30 \\
\hline Summer & Medium & Low & Low & Low & Medium & High & 15 & 45 & 40 \\
\hline Summer & Medium & Low & Low & Low & High & Low & 45 & 55 & 0 \\
\hline Summer & Medium & Low & Low & Low & High & Medium & 35 & 55 & 10 \\
\hline Summer & Medium & Low & Low & Low & High & High & 25 & 55 & 20 \\
\hline Summer & Medium & Low & Low & Medium & Low & Low & 25 & 45 & 30 \\
\hline Summer & Medium & Low & Low & Medium & Low & Medium & 15 & 35 & 50 \\
\hline Summer & Medium & Low & Low & Medium & Low & High & 5 & 35 & 60 \\
\hline Summer & Medium & Low & Low & Medium & Medium & Low & 30 & 50 & 20 \\
\hline Summer & Medium & Low & Low & Medium & Medium & Medium & 20 & 40 & 40 \\
\hline Summer & Medium & Low & Low & Medium & Medium & High & 10 & 40 & 50 \\
\hline Summer & Medium & Low & Low & Medium & High & Low & 40 & 60 & 0 \\
\hline Summer & Medium & Low & Low & Medium & High & Medium & 30 & 50 & 20 \\
\hline Summer & Medium & Low & Low & Medium & High & High & 20 & 50 & 30 \\
\hline Summer & Medium & Low & Low & High & Low & Low & 25 & 40 & 35 \\
\hline Summer & Medium & Low & Low & High & Low & Medium & 15 & 30 & 55 \\
\hline Summer & Medium & Low & Low & High & Low & High & 5 & 30 & 65 \\
\hline Summer & Medium & Low & Low & High & Medium & Low & 30 & 45 & 25 \\
\hline Summer & Medium & Low & Low & High & Medium & Medium & 20 & 35 & 45 \\
\hline Summer & Medium & Low & Low & High & Medium & High & 10 & 35 & 55 \\
\hline Summer & Medium & Low & Low & High & High & Low & 40 & 55 & 5 \\
\hline Summer & Medium & Low & Low & High & High & Medium & 30 & 45 & 25 \\
\hline Summer & Medium & Low & Low & High & High & High & 20 & 45 & 35 \\
\hline Summer & Medium & Low & Medium & Low & Low & Low & 25 & 45 & 30 \\
\hline Summer & Medium & Low & Medium & Low & Low & Medium & 15 & 35 & 50 \\
\hline Summer & Medium & Low & Medium & Low & Low & High & 5 & 35 & 60 \\
\hline Summer & Medium & Low & Medium & Low & Medium & Low & 30 & 50 & 20 \\
\hline Summer & Medium & Low & Medium & Low & Medium & Medium & 20 & 40 & 40 \\
\hline Summer & Medium & Low & Medium & Low & Medium & High & 10 & 40 & 50 \\
\hline Summer & Medium & Low & Medium & Low & High & Low & 40 & 60 & 0 \\
\hline Summer & Medium & Low & Medium & Low & High & Medium & 30 & 50 & 20 \\
\hline Summer & Medium & Low & Medium & Low & High & High & 20 & 50 & 30 \\
\hline Summer & Medium & Low & Medium & Medium & Low & Low & 20 & 40 & 40 \\
\hline Summer & Medium & Low & Medium & Medium & Low & Medium & 10 & 30 & 60 \\
\hline Summer & Medium & Low & Medium & Medium & Low & High & 0 & 30 & 70 \\
\hline Summer & Medium & Low & Medium & Medium & Medium & Low & 25 & 45 & 30 \\
\hline Summer & Medium & Low & Medium & Medium & Medium & Medium & 15 & 35 & 50 \\
\hline Summer & Medium & Low & Medium & Medium & Medium & High & 5 & 35 & 60 \\
\hline Summer & Medium & Low & Medium & Medium & High & Low & 35 & 55 & 10 \\
\hline Summer & Medium & Low & Medium & Medium & High & Medium & 25 & 45 & 30 \\
\hline
\end{tabular}




\begin{tabular}{|c|c|c|c|c|c|c|c|c|c|}
\hline \multicolumn{7}{|c|}{ Inputs } & \multicolumn{3}{|c|}{ Habitat quality } \\
\hline $\begin{array}{c}\text { Water } \\
\text { availability }\end{array}$ & Emergent & Floating & Terrestrial & $\begin{array}{l}\text { Winter } \\
\text { refuge }\end{array}$ & Submerged & $\begin{array}{l}\text { Water } \\
\text { quality }\end{array}$ & Low & Average & High \\
\hline Summer & Medium & Low & Medium & Medium & High & High & 15 & 45 & 40 \\
\hline Summer & Medium & Low & Medium & High & Low & Low & 20 & 35 & 45 \\
\hline Summer & Medium & Low & Medium & High & Low & Medium & 10 & 25 & 65 \\
\hline Summer & Medium & Low & Medium & High & Low & High & 0 & 25 & 75 \\
\hline Summer & Medium & Low & Medium & High & Medium & Low & 25 & 40 & 35 \\
\hline Summer & Medium & Low & Medium & High & Medium & Medium & 15 & 30 & 55 \\
\hline Summer & Medium & Low & Medium & High & Medium & High & 5 & 30 & 65 \\
\hline Summer & Medium & Low & Medium & High & High & Low & 35 & 50 & 15 \\
\hline Summer & Medium & Low & Medium & High & High & Medium & 25 & 40 & 35 \\
\hline Summer & Medium & Low & Medium & High & High & High & 15 & 40 & 45 \\
\hline Summer & Medium & Low & High & Low & Low & Low & 25 & 40 & 35 \\
\hline Summer & Medium & Low & High & Low & Low & Medium & 15 & 30 & 55 \\
\hline Summer & Medium & Low & High & Low & Low & High & 5 & 30 & 65 \\
\hline Summer & Medium & Low & High & Low & Medium & Low & 30 & 45 & 25 \\
\hline Summer & Medium & Low & High & Low & Medium & Medium & 20 & 35 & 45 \\
\hline Summer & Medium & Low & High & Low & Medium & High & 10 & 35 & 55 \\
\hline Summer & Medium & Low & High & Low & High & Low & 40 & 55 & 5 \\
\hline Summer & Medium & Low & High & Low & High & Medium & 30 & 45 & 25 \\
\hline Summer & Medium & Low & High & Low & High & High & 20 & 45 & 35 \\
\hline Summer & Medium & Low & High & Medium & Low & Low & 20 & 35 & 45 \\
\hline Summer & Medium & Low & High & Medium & Low & Medium & 10 & 25 & 65 \\
\hline Summer & Medium & Low & High & Medium & Low & High & 0 & 25 & 75 \\
\hline Summer & Medium & Low & High & Medium & Medium & Low & 25 & 40 & 35 \\
\hline Summer & Medium & Low & High & Medium & Medium & Medium & 15 & 30 & 55 \\
\hline Summer & Medium & Low & High & Medium & Medium & High & 5 & 30 & 65 \\
\hline Summer & Medium & Low & High & Medium & High & Low & 35 & 50 & 15 \\
\hline Summer & Medium & Low & High & Medium & High & Medium & 25 & 40 & 35 \\
\hline Summer & Medium & Low & High & Medium & High & High & 15 & 40 & 45 \\
\hline Summer & Medium & Low & High & High & Low & Low & 20 & 30 & 50 \\
\hline Summer & Medium & Low & High & High & Low & Medium & 10 & 20 & 70 \\
\hline Summer & Medium & Low & High & High & Low & High & 0 & 20 & 80 \\
\hline Summer & Medium & Low & High & High & Medium & Low & 25 & 35 & 40 \\
\hline Summer & Medium & Low & High & High & Medium & Medium & 15 & 25 & 60 \\
\hline Summer & Medium & Low & High & High & Medium & High & 5 & 25 & 70 \\
\hline Summer & Medium & Low & High & High & High & Low & 35 & 45 & 20 \\
\hline Summer & Medium & Low & High & High & High & Medium & 25 & 35 & 40 \\
\hline Summer & Medium & Low & High & High & High & High & 15 & 35 & 50 \\
\hline Summer & Medium & Medium & Low & Low & Low & Low & 35 & 50 & 15 \\
\hline Summer & Medium & Medium & Low & Low & Low & Medium & 25 & 40 & 35 \\
\hline Summer & Medium & Medium & Low & Low & Low & High & 15 & 40 & 45 \\
\hline
\end{tabular}




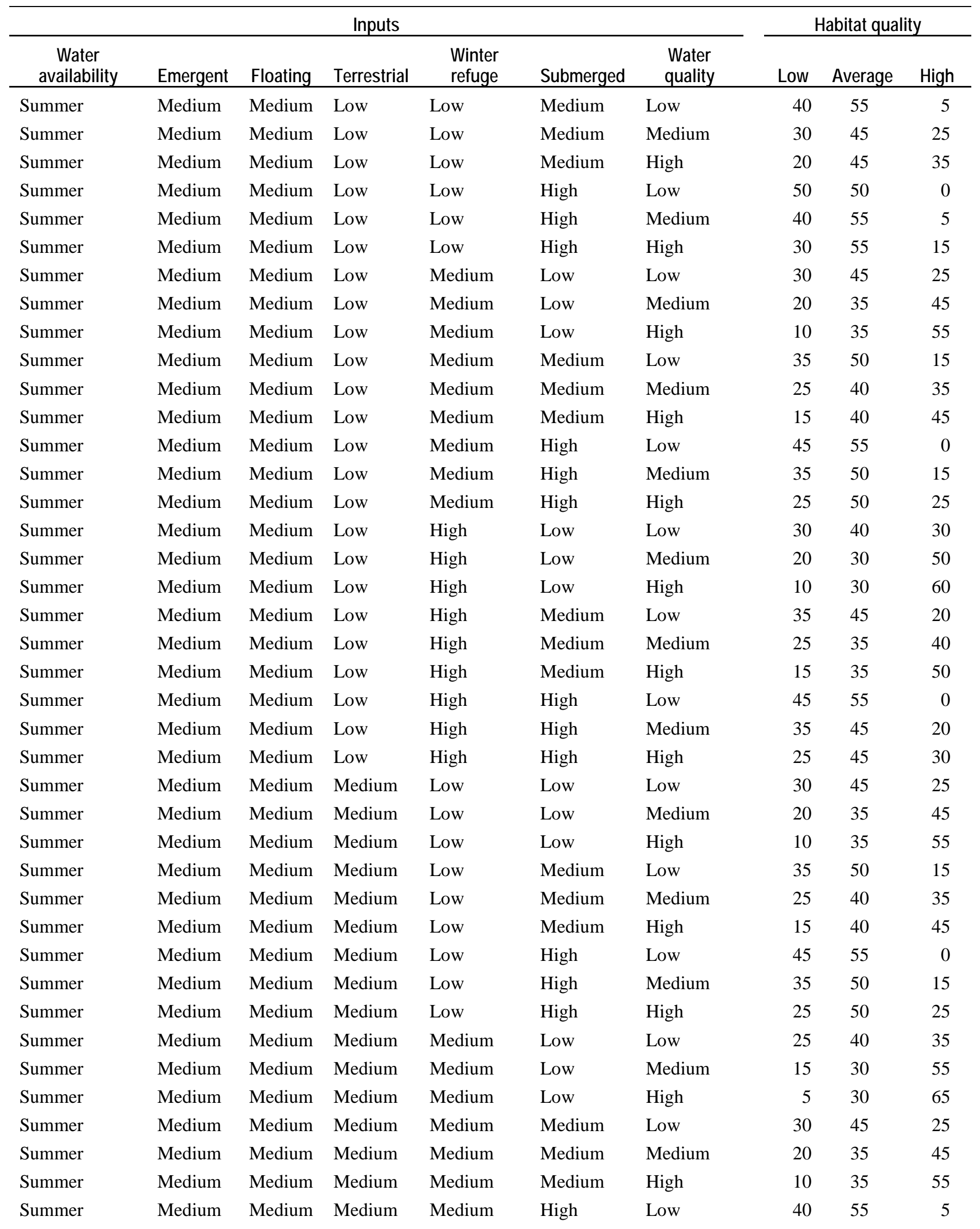




\begin{tabular}{|c|c|c|c|c|c|c|c|c|c|}
\hline \multicolumn{7}{|c|}{ Inputs } & \multicolumn{3}{|c|}{ Habitat quality } \\
\hline $\begin{array}{c}\text { Water } \\
\text { availability }\end{array}$ & Emergent & Floating & Terrestrial & $\begin{array}{l}\text { Winter } \\
\text { refuge }\end{array}$ & Submerged & $\begin{array}{l}\text { Water } \\
\text { quality }\end{array}$ & Low & Average & High \\
\hline Summer & Medium & Medium & Medium & Medium & High & Medium & 30 & 45 & 25 \\
\hline Summer & Medium & Medium & Medium & Medium & High & High & 20 & 45 & 35 \\
\hline Summer & Medium & Medium & Medium & High & Low & Low & 25 & 35 & 40 \\
\hline Summer & Medium & Medium & Medium & High & Low & Medium & 15 & 25 & 60 \\
\hline Summer & Medium & Medium & Medium & High & Low & High & 5 & 25 & 70 \\
\hline Summer & Medium & Medium & Medium & High & Medium & Low & 30 & 40 & 30 \\
\hline Summer & Medium & Medium & Medium & High & Medium & Medium & 20 & 30 & 50 \\
\hline Summer & Medium & Medium & Medium & High & Medium & High & 10 & 30 & 60 \\
\hline Summer & Medium & Medium & Medium & High & High & Low & 40 & 50 & 10 \\
\hline Summer & Medium & Medium & Medium & High & High & Medium & 30 & 40 & 30 \\
\hline Summer & Medium & Medium & Medium & High & High & High & 20 & 40 & 40 \\
\hline Summer & Medium & Medium & High & Low & Low & Low & 30 & 40 & 30 \\
\hline Summer & Medium & Medium & High & Low & Low & Medium & 20 & 30 & 50 \\
\hline Summer & Medium & Medium & High & Low & Low & High & 10 & 30 & 60 \\
\hline Summer & Medium & Medium & High & Low & Medium & Low & 35 & 45 & 20 \\
\hline Summer & Medium & Medium & High & Low & Medium & Medium & 25 & 35 & 40 \\
\hline Summer & Medium & Medium & High & Low & Medium & High & 15 & 35 & 50 \\
\hline Summer & Medium & Medium & High & Low & High & Low & 45 & 55 & 0 \\
\hline Summer & Medium & Medium & High & Low & High & Medium & 35 & 45 & 20 \\
\hline Summer & Medium & Medium & High & Low & High & High & 25 & 45 & 30 \\
\hline Summer & Medium & Medium & High & Medium & Low & Low & 25 & 35 & 40 \\
\hline Summer & Medium & Medium & High & Medium & Low & Medium & 15 & 25 & 60 \\
\hline Summer & Medium & Medium & High & Medium & Low & High & 5 & 25 & 70 \\
\hline Summer & Medium & Medium & High & Medium & Medium & Low & 30 & 40 & 30 \\
\hline Summer & Medium & Medium & High & Medium & Medium & Medium & 20 & 30 & 50 \\
\hline Summer & Medium & Medium & High & Medium & Medium & High & 10 & 30 & 60 \\
\hline Summer & Medium & Medium & High & Medium & High & Low & 40 & 50 & 10 \\
\hline Summer & Medium & Medium & High & Medium & High & Medium & 30 & 40 & 30 \\
\hline Summer & Medium & Medium & High & Medium & High & High & 20 & 40 & 40 \\
\hline Summer & Medium & Medium & High & High & Low & Low & 25 & 30 & 45 \\
\hline Summer & Medium & Medium & High & High & Low & Medium & 15 & 20 & 65 \\
\hline Summer & Medium & Medium & High & High & Low & High & 5 & 20 & 75 \\
\hline Summer & Medium & Medium & High & High & Medium & Low & 30 & 35 & 35 \\
\hline Summer & Medium & Medium & High & High & Medium & Medium & 20 & 25 & 55 \\
\hline Summer & Medium & Medium & High & High & Medium & High & 10 & 25 & 65 \\
\hline Summer & Medium & Medium & High & High & High & Low & 40 & 45 & 15 \\
\hline Summer & Medium & Medium & High & High & High & Medium & 30 & 35 & 35 \\
\hline Summer & Medium & Medium & High & High & High & High & 20 & 35 & 45 \\
\hline Summer & Medium & High & Low & Low & Low & Low & 40 & 55 & 5 \\
\hline Summer & Medium & High & Low & Low & Low & Medium & 30 & 45 & 25 \\
\hline
\end{tabular}




\begin{tabular}{|c|c|c|c|c|c|c|c|c|c|}
\hline \multicolumn{7}{|c|}{ Inputs } & \multicolumn{3}{|c|}{ Habitat quality } \\
\hline $\begin{array}{c}\text { Water } \\
\text { availability }\end{array}$ & Emergent & Floating & Terrestrial & $\begin{array}{l}\text { Winter } \\
\text { refuge }\end{array}$ & Submerged & $\begin{array}{l}\text { Water } \\
\text { quality }\end{array}$ & Low & Average & High \\
\hline Summer & Medium & High & Low & Low & Low & High & 20 & 45 & 35 \\
\hline Summer & Medium & High & Low & Low & Medium & Low & 45 & 55 & 0 \\
\hline Summer & Medium & High & Low & Low & Medium & Medium & 35 & 50 & 15 \\
\hline Summer & Medium & High & Low & Low & Medium & High & 25 & 50 & 25 \\
\hline Summer & Medium & High & Low & Low & High & Low & 55 & 45 & 0 \\
\hline Summer & Medium & High & Low & Low & High & Medium & 45 & 55 & 0 \\
\hline Summer & Medium & High & Low & Low & High & High & 35 & 60 & 5 \\
\hline Summer & Medium & High & Low & Medium & Low & Low & 35 & 50 & 15 \\
\hline Summer & Medium & High & Low & Medium & Low & Medium & 25 & 40 & 35 \\
\hline Summer & Medium & High & Low & Medium & Low & High & 15 & 40 & 45 \\
\hline Summer & Medium & High & Low & Medium & Medium & Low & 40 & 55 & 5 \\
\hline Summer & Medium & High & Low & Medium & Medium & Medium & 30 & 45 & 25 \\
\hline Summer & Medium & High & Low & Medium & Medium & High & 20 & 45 & 35 \\
\hline Summer & Medium & High & Low & Medium & High & Low & 45 & 55 & 0 \\
\hline Summer & Medium & High & Low & Medium & High & Medium & 40 & 55 & 5 \\
\hline Summer & Medium & High & Low & Medium & High & High & 30 & 55 & 15 \\
\hline Summer & Medium & High & Low & High & Low & Low & 35 & 45 & 20 \\
\hline Summer & Medium & High & Low & High & Low & Medium & 25 & 35 & 40 \\
\hline Summer & Medium & High & Low & High & Low & High & 15 & 35 & 50 \\
\hline Summer & Medium & High & Low & High & Medium & Low & 40 & 50 & 10 \\
\hline Summer & Medium & High & Low & High & Medium & Medium & 30 & 40 & 30 \\
\hline Summer & Medium & High & Low & High & Medium & High & 20 & 40 & 40 \\
\hline Summer & Medium & High & Low & High & High & Low & 45 & 55 & 0 \\
\hline Summer & Medium & High & Low & High & High & Medium & 40 & 50 & 10 \\
\hline Summer & Medium & High & Low & High & High & High & 30 & 50 & 20 \\
\hline Summer & Medium & High & Medium & Low & Low & Low & 35 & 50 & 15 \\
\hline Summer & Medium & High & Medium & Low & Low & Medium & 25 & 40 & 35 \\
\hline Summer & Medium & High & Medium & Low & Low & High & 15 & 40 & 45 \\
\hline Summer & Medium & High & Medium & Low & Medium & Low & 40 & 55 & 5 \\
\hline Summer & Medium & High & Medium & Low & Medium & Medium & 30 & 45 & 25 \\
\hline Summer & Medium & High & Medium & Low & Medium & High & 20 & 45 & 35 \\
\hline Summer & Medium & High & Medium & Low & High & Low & 45 & 55 & 0 \\
\hline Summer & Medium & High & Medium & Low & High & Medium & 40 & 55 & 5 \\
\hline Summer & Medium & High & Medium & Low & High & High & 30 & 55 & 15 \\
\hline Summer & Medium & High & Medium & Medium & Low & Low & 30 & 45 & 25 \\
\hline Summer & Medium & High & Medium & Medium & Low & Medium & 20 & 35 & 45 \\
\hline Summer & Medium & High & Medium & Medium & Low & High & 10 & 35 & 55 \\
\hline Summer & Medium & High & Medium & Medium & Medium & Low & 35 & 50 & 15 \\
\hline Summer & Medium & High & Medium & Medium & Medium & Medium & 25 & 40 & 35 \\
\hline Summer & Medium & High & Medium & Medium & Medium & High & 15 & 40 & 45 \\
\hline
\end{tabular}




\begin{tabular}{|c|c|c|c|c|c|c|c|c|c|}
\hline \multicolumn{7}{|c|}{ Inputs } & \multicolumn{3}{|c|}{ Habitat quality } \\
\hline $\begin{array}{c}\text { Water } \\
\text { availability }\end{array}$ & Emergent & Floating & Terrestrial & $\begin{array}{l}\text { Winter } \\
\text { refuge }\end{array}$ & Submerged & $\begin{array}{l}\text { Water } \\
\text { quality }\end{array}$ & Low & Average & High \\
\hline Summer & Medium & High & Medium & Medium & High & Low & 45 & 55 & 0 \\
\hline Summer & Medium & High & Medium & Medium & High & Medium & 35 & 50 & 15 \\
\hline Summer & Medium & High & Medium & Medium & High & High & 25 & 50 & 25 \\
\hline Summer & Medium & High & Medium & High & Low & Low & 30 & 40 & 30 \\
\hline Summer & Medium & High & Medium & High & Low & Medium & 20 & 30 & 50 \\
\hline Summer & Medium & High & Medium & High & Low & High & 10 & 30 & 60 \\
\hline Summer & Medium & High & Medium & High & Medium & Low & 35 & 45 & 20 \\
\hline Summer & Medium & High & Medium & High & Medium & Medium & 25 & 35 & 40 \\
\hline Summer & Medium & High & Medium & High & Medium & High & 15 & 35 & 50 \\
\hline Summer & Medium & High & Medium & High & High & Low & 45 & 55 & 0 \\
\hline Summer & Medium & High & Medium & High & High & Medium & 35 & 45 & 20 \\
\hline Summer & Medium & High & Medium & High & High & High & 25 & 45 & 30 \\
\hline Summer & Medium & High & High & Low & Low & Low & 35 & 45 & 20 \\
\hline Summer & Medium & High & High & Low & Low & Medium & 25 & 35 & 40 \\
\hline Summer & Medium & High & High & Low & Low & High & 15 & 35 & 50 \\
\hline Summer & Medium & High & High & Low & Medium & Low & 40 & 50 & 10 \\
\hline Summer & Medium & High & High & Low & Medium & Medium & 30 & 40 & 30 \\
\hline Summer & Medium & High & High & Low & Medium & High & 20 & 40 & 40 \\
\hline Summer & Medium & High & High & Low & High & Low & 45 & 55 & 0 \\
\hline Summer & Medium & High & High & Low & High & Medium & 40 & 50 & 10 \\
\hline Summer & Medium & High & High & Low & High & High & 30 & 50 & 20 \\
\hline Summer & Medium & High & High & Medium & Low & Low & 30 & 40 & 30 \\
\hline Summer & Medium & High & High & Medium & Low & Medium & 20 & 30 & 50 \\
\hline Summer & Medium & High & High & Medium & Low & High & 10 & 30 & 60 \\
\hline Summer & Medium & High & High & Medium & Medium & Low & 35 & 45 & 20 \\
\hline Summer & Medium & High & High & Medium & Medium & Medium & 25 & 35 & 40 \\
\hline Summer & Medium & High & High & Medium & Medium & High & 15 & 35 & 50 \\
\hline Summer & Medium & High & High & Medium & High & Low & 45 & 55 & 0 \\
\hline Summer & Medium & High & High & Medium & High & Medium & 35 & 45 & 20 \\
\hline Summer & Medium & High & High & Medium & High & High & 25 & 45 & 30 \\
\hline Summer & Medium & High & High & High & Low & Low & 30 & 35 & 35 \\
\hline Summer & Medium & High & High & High & Low & Medium & 20 & 25 & 55 \\
\hline Summer & Medium & High & High & High & Low & High & 10 & 25 & 65 \\
\hline Summer & Medium & High & High & High & Medium & Low & 35 & 40 & 25 \\
\hline Summer & Medium & High & High & High & Medium & Medium & 25 & 30 & 45 \\
\hline Summer & Medium & High & High & High & Medium & High & 15 & 30 & 55 \\
\hline Summer & Medium & High & High & High & High & Low & 45 & 50 & 5 \\
\hline Summer & Medium & High & High & High & High & Medium & 35 & 40 & 25 \\
\hline Summer & Medium & High & High & High & High & High & 25 & 40 & 35 \\
\hline Summer & High & Low & Low & Low & Low & Low & 30 & 55 & 15 \\
\hline
\end{tabular}




\begin{tabular}{|c|c|c|c|c|c|c|c|c|c|}
\hline \multicolumn{7}{|c|}{ Inputs } & \multicolumn{3}{|c|}{ Habitat quality } \\
\hline $\begin{array}{c}\text { Water } \\
\text { availability }\end{array}$ & Emergent & Floating & Terrestrial & $\begin{array}{l}\text { Winter } \\
\text { refuge }\end{array}$ & Submerged & $\begin{array}{l}\text { Water } \\
\text { quality }\end{array}$ & Low & Average & High \\
\hline Summer & High & Low & Low & Low & Low & Medium & 20 & 45 & 35 \\
\hline Summer & High & Low & Low & Low & Low & High & 10 & 45 & 45 \\
\hline Summer & High & Low & Low & Low & Medium & Low & 35 & 60 & 5 \\
\hline Summer & High & Low & Low & Low & Medium & Medium & 25 & 50 & 25 \\
\hline Summer & High & Low & Low & Low & Medium & High & 15 & 50 & 35 \\
\hline Summer & High & Low & Low & Low & High & Low & 45 & 55 & 0 \\
\hline Summer & High & Low & Low & Low & High & Medium & 35 & 60 & 5 \\
\hline Summer & High & Low & Low & Low & High & High & 25 & 60 & 15 \\
\hline Summer & High & Low & Low & Medium & Low & Low & 25 & 50 & 25 \\
\hline Summer & High & Low & Low & Medium & Low & Medium & 15 & 40 & 45 \\
\hline Summer & High & Low & Low & Medium & Low & High & 5 & 40 & 55 \\
\hline Summer & High & Low & Low & Medium & Medium & Low & 30 & 55 & 15 \\
\hline Summer & High & Low & Low & Medium & Medium & Medium & 20 & 45 & 35 \\
\hline Summer & High & Low & Low & Medium & Medium & High & 10 & 45 & 45 \\
\hline Summer & High & Low & Low & Medium & High & Low & 40 & 60 & 0 \\
\hline Summer & High & Low & Low & Medium & High & Medium & 30 & 55 & 15 \\
\hline Summer & High & Low & Low & Medium & High & High & 20 & 55 & 25 \\
\hline Summer & High & Low & Low & High & Low & Low & 25 & 45 & 30 \\
\hline Summer & High & Low & Low & High & Low & Medium & 15 & 35 & 50 \\
\hline Summer & High & Low & Low & High & Low & High & 5 & 35 & 60 \\
\hline Summer & High & Low & Low & High & Medium & Low & 30 & 50 & 20 \\
\hline Summer & High & Low & Low & High & Medium & Medium & 20 & 40 & 40 \\
\hline Summer & High & Low & Low & High & Medium & High & 10 & 40 & 50 \\
\hline Summer & High & Low & Low & High & High & Low & 40 & 60 & 0 \\
\hline Summer & High & Low & Low & High & High & Medium & 30 & 50 & 20 \\
\hline Summer & High & Low & Low & High & High & High & 20 & 50 & 30 \\
\hline Summer & High & Low & Medium & Low & Low & Low & 25 & 50 & 25 \\
\hline Summer & High & Low & Medium & Low & Low & Medium & 15 & 40 & 45 \\
\hline Summer & High & Low & Medium & Low & Low & High & 5 & 40 & 55 \\
\hline Summer & High & Low & Medium & Low & Medium & Low & 30 & 55 & 15 \\
\hline Summer & High & Low & Medium & Low & Medium & Medium & 20 & 45 & 35 \\
\hline Summer & High & Low & Medium & Low & Medium & High & 10 & 45 & 45 \\
\hline Summer & High & Low & Medium & Low & High & Low & 40 & 60 & 0 \\
\hline Summer & High & Low & Medium & Low & High & Medium & 30 & 55 & 15 \\
\hline Summer & High & Low & Medium & Low & High & High & 20 & 55 & 25 \\
\hline Summer & High & Low & Medium & Medium & Low & Low & 20 & 45 & 35 \\
\hline Summer & High & Low & Medium & Medium & Low & Medium & 10 & 35 & 55 \\
\hline Summer & High & Low & Medium & Medium & Low & High & 0 & 35 & 65 \\
\hline Summer & High & Low & Medium & Medium & Medium & Low & 25 & 50 & 25 \\
\hline Summer & High & Low & Medium & Medium & Medium & Medium & 15 & 40 & 45 \\
\hline
\end{tabular}




\begin{tabular}{|c|c|c|c|c|c|c|c|c|c|}
\hline \multicolumn{7}{|c|}{ Inputs } & \multicolumn{3}{|c|}{ Habitat quality } \\
\hline $\begin{array}{c}\text { Water } \\
\text { availability }\end{array}$ & Emergent & Floating & Terrestrial & $\begin{array}{l}\text { Winter } \\
\text { refuge }\end{array}$ & Submerged & $\begin{array}{l}\text { Water } \\
\text { quality }\end{array}$ & Low & Average & High \\
\hline Summer & High & Low & Medium & Medium & Medium & High & 5 & 40 & 55 \\
\hline Summer & High & Low & Medium & Medium & High & Low & 35 & 60 & 5 \\
\hline Summer & High & Low & Medium & Medium & High & Medium & 25 & 50 & 25 \\
\hline Summer & High & Low & Medium & Medium & High & High & 15 & 50 & 35 \\
\hline Summer & High & Low & Medium & High & Low & Low & 20 & 40 & 40 \\
\hline Summer & High & Low & Medium & High & Low & Medium & 10 & 30 & 60 \\
\hline Summer & High & Low & Medium & High & Low & High & 0 & 30 & 70 \\
\hline Summer & High & Low & Medium & High & Medium & Low & 25 & 45 & 30 \\
\hline Summer & High & Low & Medium & High & Medium & Medium & 15 & 35 & 50 \\
\hline Summer & High & Low & Medium & High & Medium & High & 5 & 35 & 60 \\
\hline Summer & High & Low & Medium & High & High & Low & 35 & 55 & 10 \\
\hline Summer & High & Low & Medium & High & High & Medium & 25 & 45 & 30 \\
\hline Summer & High & Low & Medium & High & High & High & 15 & 45 & 40 \\
\hline Summer & High & Low & High & Low & Low & Low & 25 & 45 & 30 \\
\hline Summer & High & Low & High & Low & Low & Medium & 15 & 35 & 50 \\
\hline Summer & High & Low & High & Low & Low & High & 5 & 35 & 60 \\
\hline Summer & High & Low & High & Low & Medium & Low & 30 & 50 & 20 \\
\hline Summer & High & Low & High & Low & Medium & Medium & 20 & 40 & 40 \\
\hline Summer & High & Low & High & Low & Medium & High & 10 & 40 & 50 \\
\hline Summer & High & Low & High & Low & High & Low & 40 & 60 & 0 \\
\hline Summer & High & Low & High & Low & High & Medium & 30 & 50 & 20 \\
\hline Summer & High & Low & High & Low & High & High & 20 & 50 & 30 \\
\hline Summer & High & Low & High & Medium & Low & Low & 20 & 40 & 40 \\
\hline Summer & High & Low & High & Medium & Low & Medium & 10 & 30 & 60 \\
\hline Summer & High & Low & High & Medium & Low & High & 0 & 30 & 70 \\
\hline Summer & High & Low & High & Medium & Medium & Low & 25 & 45 & 30 \\
\hline Summer & High & Low & High & Medium & Medium & Medium & 15 & 35 & 50 \\
\hline Summer & High & Low & High & Medium & Medium & High & 5 & 35 & 60 \\
\hline Summer & High & Low & High & Medium & High & Low & 35 & 55 & 10 \\
\hline Summer & High & Low & High & Medium & High & Medium & 25 & 45 & 30 \\
\hline Summer & High & Low & High & Medium & High & High & 15 & 45 & 40 \\
\hline Summer & High & Low & High & High & Low & Low & 20 & 35 & 45 \\
\hline Summer & High & Low & High & High & Low & Medium & 10 & 25 & 65 \\
\hline Summer & High & Low & High & High & Low & High & 0 & 25 & 75 \\
\hline Summer & High & Low & High & High & Medium & Low & 25 & 40 & 35 \\
\hline Summer & High & Low & High & High & Medium & Medium & 15 & 30 & 55 \\
\hline Summer & High & Low & High & High & Medium & High & 5 & 30 & 65 \\
\hline Summer & High & Low & High & High & High & Low & 35 & 50 & 15 \\
\hline Summer & High & Low & High & High & High & Medium & 25 & 40 & 35 \\
\hline Summer & High & Low & High & High & High & High & 15 & 40 & 45 \\
\hline
\end{tabular}




\begin{tabular}{|c|c|c|c|c|c|c|c|c|c|}
\hline \multicolumn{7}{|c|}{ Inputs } & \multicolumn{3}{|c|}{ Habitat quality } \\
\hline $\begin{array}{c}\text { Water } \\
\text { availability }\end{array}$ & Emergent & Floating & Terrestrial & $\begin{array}{l}\text { Winter } \\
\text { refuge }\end{array}$ & Submerged & $\begin{array}{l}\text { Water } \\
\text { quality }\end{array}$ & Low & Average & High \\
\hline Summer & High & Medium & Low & Low & Low & Low & 35 & 55 & 10 \\
\hline Summer & High & Medium & Low & Low & Low & Medium & 25 & 45 & 30 \\
\hline Summer & High & Medium & Low & Low & Low & High & 15 & 45 & 40 \\
\hline Summer & High & Medium & Low & Low & Medium & Low & 40 & 60 & 0 \\
\hline Summer & High & Medium & Low & Low & Medium & Medium & 30 & 50 & 20 \\
\hline Summer & High & Medium & Low & Low & Medium & High & 20 & 50 & 30 \\
\hline Summer & High & Medium & Low & Low & High & Low & 50 & 50 & 0 \\
\hline Summer & High & Medium & Low & Low & High & Medium & 40 & 60 & 0 \\
\hline Summer & High & Medium & Low & Low & High & High & 30 & 60 & 10 \\
\hline Summer & High & Medium & Low & Medium & Low & Low & 30 & 50 & 20 \\
\hline Summer & High & Medium & Low & Medium & Low & Medium & 20 & 40 & 40 \\
\hline Summer & High & Medium & Low & Medium & Low & High & 10 & 40 & 50 \\
\hline Summer & High & Medium & Low & Medium & Medium & Low & 35 & 55 & 10 \\
\hline Summer & High & Medium & Low & Medium & Medium & Medium & 25 & 45 & 30 \\
\hline Summer & High & Medium & Low & Medium & Medium & High & 15 & 45 & 40 \\
\hline Summer & High & Medium & Low & Medium & High & Low & 45 & 55 & 0 \\
\hline Summer & High & Medium & Low & Medium & High & Medium & 35 & 55 & 10 \\
\hline Summer & High & Medium & Low & Medium & High & High & 25 & 55 & 20 \\
\hline Summer & High & Medium & Low & High & Low & Low & 30 & 45 & 25 \\
\hline Summer & High & Medium & Low & High & Low & Medium & 20 & 35 & 45 \\
\hline Summer & High & Medium & Low & High & Low & High & 10 & 35 & 55 \\
\hline Summer & High & Medium & Low & High & Medium & Low & 35 & 50 & 15 \\
\hline Summer & High & Medium & Low & High & Medium & Medium & 25 & 40 & 35 \\
\hline Summer & High & Medium & Low & High & Medium & High & 15 & 40 & 45 \\
\hline Summer & High & Medium & Low & High & High & Low & 45 & 55 & 0 \\
\hline Summer & High & Medium & Low & High & High & Medium & 35 & 50 & 15 \\
\hline Summer & High & Medium & Low & High & High & High & 25 & 50 & 25 \\
\hline Summer & High & Medium & Medium & Low & Low & Low & 30 & 50 & 20 \\
\hline Summer & High & Medium & Medium & Low & Low & Medium & 20 & 40 & 40 \\
\hline Summer & High & Medium & Medium & Low & Low & High & 10 & 40 & 50 \\
\hline Summer & High & Medium & Medium & Low & Medium & Low & 35 & 55 & 10 \\
\hline Summer & High & Medium & Medium & Low & Medium & Medium & 25 & 45 & 30 \\
\hline Summer & High & Medium & Medium & Low & Medium & High & 15 & 45 & 40 \\
\hline Summer & High & Medium & Medium & Low & High & Low & 45 & 55 & 0 \\
\hline Summer & High & Medium & Medium & Low & High & Medium & 35 & 55 & 10 \\
\hline Summer & High & Medium & Medium & Low & High & High & 25 & 55 & 20 \\
\hline Summer & High & Medium & Medium & Medium & Low & Low & 25 & 45 & 30 \\
\hline Summer & High & Medium & Medium & Medium & Low & Medium & 15 & 35 & 50 \\
\hline Summer & High & Medium & Medium & Medium & Low & High & 5 & 35 & 60 \\
\hline Summer & High & Medium & Medium & Medium & Medium & Low & 30 & 50 & 20 \\
\hline
\end{tabular}




\begin{tabular}{|c|c|c|c|c|c|c|c|c|c|}
\hline \multicolumn{7}{|c|}{ Inputs } & \multicolumn{3}{|c|}{ Habitat quality } \\
\hline $\begin{array}{c}\text { Water } \\
\text { availability }\end{array}$ & Emergent & Floating & Terrestrial & $\begin{array}{l}\text { Winter } \\
\text { refuge }\end{array}$ & Submerged & $\begin{array}{l}\text { Water } \\
\text { quality }\end{array}$ & Low & Average & High \\
\hline Summer & High & Medium & Medium & Medium & Medium & Medium & 20 & 40 & 40 \\
\hline Summer & High & Medium & Medium & Medium & Medium & High & 10 & 40 & 50 \\
\hline Summer & High & Medium & Medium & Medium & High & Low & 40 & 60 & 0 \\
\hline Summer & High & Medium & Medium & Medium & High & Medium & 30 & 50 & 20 \\
\hline Summer & High & Medium & Medium & Medium & High & High & 20 & 50 & 30 \\
\hline Summer & High & Medium & Medium & High & Low & Low & 25 & 40 & 35 \\
\hline Summer & High & Medium & Medium & High & Low & Medium & 15 & 30 & 55 \\
\hline Summer & High & Medium & Medium & High & Low & High & 5 & 30 & 65 \\
\hline Summer & High & Medium & Medium & High & Medium & Low & 30 & 45 & 25 \\
\hline Summer & High & Medium & Medium & High & Medium & Medium & 20 & 35 & 45 \\
\hline Summer & High & Medium & Medium & High & Medium & High & 10 & 35 & 55 \\
\hline Summer & High & Medium & Medium & High & High & Low & 40 & 55 & 5 \\
\hline Summer & High & Medium & Medium & High & High & Medium & 30 & 45 & 25 \\
\hline Summer & High & Medium & Medium & High & High & High & 20 & 45 & 35 \\
\hline Summer & High & Medium & High & Low & Low & Low & 30 & 45 & 25 \\
\hline Summer & High & Medium & High & Low & Low & Medium & 20 & 35 & 45 \\
\hline Summer & High & Medium & High & Low & Low & High & 10 & 35 & 55 \\
\hline Summer & High & Medium & High & Low & Medium & Low & 35 & 50 & 15 \\
\hline Summer & High & Medium & High & Low & Medium & Medium & 25 & 40 & 35 \\
\hline Summer & High & Medium & High & Low & Medium & High & 15 & 40 & 45 \\
\hline Summer & High & Medium & High & Low & High & Low & 45 & 55 & 0 \\
\hline Summer & High & Medium & High & Low & High & Medium & 35 & 50 & 15 \\
\hline Summer & High & Medium & High & Low & High & High & 25 & 50 & 25 \\
\hline Summer & High & Medium & High & Medium & Low & Low & 25 & 40 & 35 \\
\hline Summer & High & Medium & High & Medium & Low & Medium & 15 & 30 & 55 \\
\hline Summer & High & Medium & High & Medium & Low & High & 5 & 30 & 65 \\
\hline Summer & High & Medium & High & Medium & Medium & Low & 30 & 45 & 25 \\
\hline Summer & High & Medium & High & Medium & Medium & Medium & 20 & 35 & 45 \\
\hline Summer & High & Medium & High & Medium & Medium & High & 10 & 35 & 55 \\
\hline Summer & High & Medium & High & Medium & High & Low & 40 & 55 & 5 \\
\hline Summer & High & Medium & High & Medium & High & Medium & 30 & 45 & 25 \\
\hline Summer & High & Medium & High & Medium & High & High & 20 & 45 & 35 \\
\hline Summer & High & Medium & High & High & Low & Low & 25 & 35 & 40 \\
\hline Summer & High & Medium & High & High & Low & Medium & 15 & 25 & 60 \\
\hline Summer & High & Medium & High & High & Low & High & 5 & 25 & 70 \\
\hline Summer & High & Medium & High & High & Medium & Low & 30 & 40 & 30 \\
\hline Summer & High & Medium & High & High & Medium & Medium & 20 & 30 & 50 \\
\hline Summer & High & Medium & High & High & Medium & High & 10 & 30 & 60 \\
\hline Summer & High & Medium & High & High & High & Low & 40 & 50 & 10 \\
\hline Summer & High & Medium & High & High & High & Medium & 30 & 40 & 30 \\
\hline
\end{tabular}




\begin{tabular}{|c|c|c|c|c|c|c|c|c|c|}
\hline \multicolumn{7}{|c|}{ Inputs } & \multicolumn{3}{|c|}{ Habitat quality } \\
\hline $\begin{array}{c}\text { Water } \\
\text { availability }\end{array}$ & Emergent & Floating & Terrestrial & $\begin{array}{l}\text { Winter } \\
\text { refuge }\end{array}$ & Submerged & $\begin{array}{l}\text { Water } \\
\text { quality }\end{array}$ & Low & Average & High \\
\hline Summer & High & Medium & High & High & High & High & 20 & 40 & 40 \\
\hline Summer & High & High & Low & Low & Low & Low & 40 & 60 & 0 \\
\hline Summer & High & High & Low & Low & Low & Medium & 30 & 50 & 20 \\
\hline Summer & High & High & Low & Low & Low & High & 20 & 50 & 30 \\
\hline Summer & High & High & Low & Low & Medium & Low & 45 & 55 & 0 \\
\hline Summer & High & High & Low & Low & Medium & Medium & 35 & 55 & 10 \\
\hline Summer & High & High & Low & Low & Medium & High & 25 & 55 & 20 \\
\hline Summer & High & High & Low & Low & High & Low & 55 & 45 & 0 \\
\hline Summer & High & High & Low & Low & High & Medium & 45 & 55 & 0 \\
\hline Summer & High & High & Low & Low & High & High & 35 & 65 & 0 \\
\hline Summer & High & High & Low & Medium & Low & Low & 35 & 55 & 10 \\
\hline Summer & High & High & Low & Medium & Low & Medium & 25 & 45 & 30 \\
\hline Summer & High & High & Low & Medium & Low & High & 15 & 45 & 40 \\
\hline Summer & High & High & Low & Medium & Medium & Low & 40 & 60 & 0 \\
\hline Summer & High & High & Low & Medium & Medium & Medium & 30 & 50 & 20 \\
\hline Summer & High & High & Low & Medium & Medium & High & 20 & 50 & 30 \\
\hline Summer & High & High & Low & Medium & High & Low & 45 & 55 & 0 \\
\hline Summer & High & High & Low & Medium & High & Medium & 40 & 60 & 0 \\
\hline Summer & High & High & Low & Medium & High & High & 30 & 60 & 10 \\
\hline Summer & High & High & Low & High & Low & Low & 35 & 50 & 15 \\
\hline Summer & High & High & Low & High & Low & Medium & 25 & 40 & 35 \\
\hline Summer & High & High & Low & High & Low & High & 15 & 40 & 45 \\
\hline Summer & High & High & Low & High & Medium & Low & 40 & 55 & 5 \\
\hline Summer & High & High & Low & High & Medium & Medium & 30 & 45 & 25 \\
\hline Summer & High & High & Low & High & Medium & High & 20 & 45 & 35 \\
\hline Summer & High & High & Low & High & High & Low & 45 & 55 & 0 \\
\hline Summer & High & High & Low & High & High & Medium & 40 & 55 & 5 \\
\hline Summer & High & High & Low & High & High & High & 30 & 55 & 15 \\
\hline Summer & High & High & Medium & Low & Low & Low & 35 & 55 & 10 \\
\hline Summer & High & High & Medium & Low & Low & Medium & 25 & 45 & 30 \\
\hline Summer & High & High & Medium & Low & Low & High & 15 & 45 & 40 \\
\hline Summer & High & High & Medium & Low & Medium & Low & 40 & 60 & 0 \\
\hline Summer & High & High & Medium & Low & Medium & Medium & 30 & 50 & 20 \\
\hline Summer & High & High & Medium & Low & Medium & High & 20 & 50 & 30 \\
\hline Summer & High & High & Medium & Low & High & Low & 45 & 55 & 0 \\
\hline Summer & High & High & Medium & Low & High & Medium & 40 & 60 & 0 \\
\hline Summer & High & High & Medium & Low & High & High & 30 & 60 & 10 \\
\hline Summer & High & High & Medium & Medium & Low & Low & 30 & 50 & 20 \\
\hline Summer & High & High & Medium & Medium & Low & Medium & 20 & 40 & 40 \\
\hline Summer & High & High & Medium & Medium & Low & High & 10 & 40 & 50 \\
\hline
\end{tabular}




\begin{tabular}{|c|c|c|c|c|c|c|c|c|c|}
\hline \multicolumn{7}{|c|}{ Inputs } & \multicolumn{3}{|c|}{ Habitat quality } \\
\hline $\begin{array}{c}\text { Water } \\
\text { availability }\end{array}$ & Emergent & Floating & Terrestrial & $\begin{array}{l}\text { Winter } \\
\text { refuge }\end{array}$ & Submerged & $\begin{array}{l}\text { Water } \\
\text { quality }\end{array}$ & Low & Average & High \\
\hline Summer & High & High & Medium & Medium & Medium & Low & 35 & 55 & 10 \\
\hline Summer & High & High & Medium & Medium & Medium & Medium & 25 & 45 & 30 \\
\hline Summer & High & High & Medium & Medium & Medium & High & 15 & 45 & 40 \\
\hline Summer & High & High & Medium & Medium & High & Low & 45 & 55 & 0 \\
\hline Summer & High & High & Medium & Medium & High & Medium & 35 & 55 & 10 \\
\hline Summer & High & High & Medium & Medium & High & High & 25 & 55 & 20 \\
\hline Summer & High & High & Medium & High & Low & Low & 30 & 45 & 25 \\
\hline Summer & High & High & Medium & High & Low & Medium & 20 & 35 & 45 \\
\hline Summer & High & High & Medium & High & Low & High & 10 & 35 & 55 \\
\hline Summer & High & High & Medium & High & Medium & Low & 35 & 50 & 15 \\
\hline Summer & High & High & Medium & High & Medium & Medium & 25 & 40 & 35 \\
\hline Summer & High & High & Medium & High & Medium & High & 15 & 40 & 45 \\
\hline Summer & High & High & Medium & High & High & Low & 45 & 55 & 0 \\
\hline Summer & High & High & Medium & High & High & Medium & 35 & 50 & 15 \\
\hline Summer & High & High & Medium & High & High & High & 25 & 50 & 25 \\
\hline Summer & High & High & High & Low & Low & Low & 35 & 50 & 15 \\
\hline Summer & High & High & High & Low & Low & Medium & 25 & 40 & 35 \\
\hline Summer & High & High & High & Low & Low & High & 15 & 40 & 45 \\
\hline Summer & High & High & High & Low & Medium & Low & 40 & 55 & 5 \\
\hline Summer & High & High & High & Low & Medium & Medium & 30 & 45 & 25 \\
\hline Summer & High & High & High & Low & Medium & High & 20 & 45 & 35 \\
\hline Summer & High & High & High & Low & High & Low & 45 & 55 & 0 \\
\hline Summer & High & High & High & Low & High & Medium & 40 & 55 & 5 \\
\hline Summer & High & High & High & Low & High & High & 30 & 55 & 15 \\
\hline Summer & High & High & High & Medium & Low & Low & 30 & 45 & 25 \\
\hline Summer & High & High & High & Medium & Low & Medium & 20 & 35 & 45 \\
\hline Summer & High & High & High & Medium & Low & High & 10 & 35 & 55 \\
\hline Summer & High & High & High & Medium & Medium & Low & 35 & 50 & 15 \\
\hline Summer & High & High & High & Medium & Medium & Medium & 25 & 40 & 35 \\
\hline Summer & High & High & High & Medium & Medium & High & 15 & 40 & 45 \\
\hline Summer & High & High & High & Medium & High & Low & 45 & 55 & 0 \\
\hline Summer & High & High & High & Medium & High & Medium & 35 & 50 & 15 \\
\hline Summer & High & High & High & Medium & High & High & 25 & 50 & 25 \\
\hline Summer & High & High & High & High & Low & Low & 30 & 40 & 30 \\
\hline Summer & High & High & High & High & Low & Medium & 20 & 30 & 50 \\
\hline Summer & High & High & High & High & Low & High & 10 & 30 & 60 \\
\hline Summer & High & High & High & High & Medium & Low & 35 & 45 & 20 \\
\hline Summer & High & High & High & High & Medium & Medium & 25 & 35 & 40 \\
\hline Summer & High & High & High & High & Medium & High & 15 & 35 & 50 \\
\hline Summer & High & High & High & High & High & Low & 45 & 55 & 0 \\
\hline
\end{tabular}




\begin{tabular}{|c|c|c|c|c|c|c|c|c|c|}
\hline \multicolumn{7}{|c|}{ Inputs } & \multicolumn{3}{|c|}{ Habitat quality } \\
\hline $\begin{array}{c}\text { Water } \\
\text { availability }\end{array}$ & Emergent & Floating & Terrestrial & $\begin{array}{l}\text { Winter } \\
\text { refuge }\end{array}$ & Submerged & $\begin{array}{l}\text { Water } \\
\text { quality }\end{array}$ & Low & Average & High \\
\hline Summer & High & High & High & High & High & Medium & 35 & 45 & 20 \\
\hline Summer & High & High & High & High & High & High & 25 & 45 & 30 \\
\hline Permanent & Low & Low & Low & Low & Low & Low & 35 & 45 & 20 \\
\hline Permanent & Low & Low & Low & Low & Low & Medium & 25 & 35 & 40 \\
\hline Permanent & Low & Low & Low & Low & Low & High & 15 & 35 & 50 \\
\hline Permanent & Low & Low & Low & Low & Medium & Low & 40 & 50 & 10 \\
\hline Permanent & Low & Low & Low & Low & Medium & Medium & 30 & 40 & 30 \\
\hline Permanent & Low & Low & Low & Low & Medium & High & 20 & 40 & 40 \\
\hline Permanent & Low & Low & Low & Low & High & Low & 45 & 55 & 0 \\
\hline Permanent & Low & Low & Low & Low & High & Medium & 40 & 50 & 10 \\
\hline Permanent & Low & Low & Low & Low & High & High & 30 & 50 & 20 \\
\hline Permanent & Low & Low & Low & Medium & Low & Low & 30 & 40 & 30 \\
\hline Permanent & Low & Low & Low & Medium & Low & Medium & 20 & 30 & 50 \\
\hline Permanent & Low & Low & Low & Medium & Low & High & 10 & 30 & 60 \\
\hline Permanent & Low & Low & Low & Medium & Medium & Low & 35 & 45 & 20 \\
\hline Permanent & Low & Low & Low & Medium & Medium & Medium & 25 & 35 & 40 \\
\hline Permanent & Low & Low & Low & Medium & Medium & High & 15 & 35 & 50 \\
\hline Permanent & Low & Low & Low & Medium & High & Low & 45 & 55 & 0 \\
\hline Permanent & Low & Low & Low & Medium & High & Medium & 35 & 45 & 20 \\
\hline Permanent & Low & Low & Low & Medium & High & High & 25 & 45 & 30 \\
\hline Permanent & Low & Low & Low & High & Low & Low & 30 & 35 & 35 \\
\hline Permanent & Low & Low & Low & High & Low & Medium & 20 & 25 & 55 \\
\hline Permanent & Low & Low & Low & High & Low & High & 10 & 25 & 65 \\
\hline Permanent & Low & Low & Low & High & Medium & Low & 35 & 40 & 25 \\
\hline Permanent & Low & Low & Low & High & Medium & Medium & 25 & 30 & 45 \\
\hline Permanent & Low & Low & Low & High & Medium & High & 15 & 30 & 55 \\
\hline Permanent & Low & Low & Low & High & High & Low & 45 & 50 & 5 \\
\hline Permanent & Low & Low & Low & High & High & Medium & 35 & 40 & 25 \\
\hline Permanent & Low & Low & Low & High & High & High & 25 & 40 & 35 \\
\hline Permanent & Low & Low & Medium & Low & Low & Low & 30 & 40 & 30 \\
\hline Permanent & Low & Low & Medium & Low & Low & Medium & 20 & 30 & 50 \\
\hline Permanent & Low & Low & Medium & Low & Low & High & 10 & 30 & 60 \\
\hline Permanent & Low & Low & Medium & Low & Medium & Low & 35 & 45 & 20 \\
\hline Permanent & Low & Low & Medium & Low & Medium & Medium & 25 & 35 & 40 \\
\hline Permanent & Low & Low & Medium & Low & Medium & High & 15 & 35 & 50 \\
\hline Permanent & Low & Low & Medium & Low & High & Low & 45 & 55 & 0 \\
\hline Permanent & Low & Low & Medium & Low & High & Medium & 35 & 45 & 20 \\
\hline Permanent & Low & Low & Medium & Low & High & High & 25 & 45 & 30 \\
\hline Permanent & Low & Low & Medium & Medium & Low & Low & 25 & 35 & 40 \\
\hline Permanent & Low & Low & Medium & Medium & Low & Medium & 15 & 25 & 60 \\
\hline
\end{tabular}




\begin{tabular}{|c|c|c|c|c|c|c|c|c|c|}
\hline \multicolumn{7}{|c|}{ Inputs } & \multicolumn{3}{|c|}{ Habitat quality } \\
\hline $\begin{array}{c}\text { Water } \\
\text { availability }\end{array}$ & Emergent & Floating & Terrestrial & $\begin{array}{l}\text { Winter } \\
\text { refuge }\end{array}$ & Submerged & $\begin{array}{l}\text { Water } \\
\text { quality }\end{array}$ & Low & Average & High \\
\hline Permanent & Low & Low & Medium & Medium & Low & High & 5 & 25 & 70 \\
\hline Permanent & Low & Low & Medium & Medium & Medium & Low & 30 & 40 & 30 \\
\hline Permanent & Low & Low & Medium & Medium & Medium & Medium & 20 & 30 & 50 \\
\hline Permanent & Low & Low & Medium & Medium & Medium & High & 10 & 30 & 60 \\
\hline Permanent & Low & Low & Medium & Medium & High & Low & 40 & 50 & 10 \\
\hline Permanent & Low & Low & Medium & Medium & High & Medium & 30 & 40 & 30 \\
\hline Permanent & Low & Low & Medium & Medium & High & High & 20 & 40 & 40 \\
\hline Permanent & Low & Low & Medium & High & Low & Low & 25 & 30 & 45 \\
\hline Permanent & Low & Low & Medium & High & Low & Medium & 15 & 20 & 65 \\
\hline Permanent & Low & Low & Medium & High & Low & High & 5 & 20 & 75 \\
\hline Permanent & Low & Low & Medium & High & Medium & Low & 30 & 35 & 35 \\
\hline Permanent & Low & Low & Medium & High & Medium & Medium & 20 & 25 & 55 \\
\hline Permanent & Low & Low & Medium & High & Medium & High & 10 & 25 & 65 \\
\hline Permanent & Low & Low & Medium & High & High & Low & 40 & 45 & 15 \\
\hline Permanent & Low & Low & Medium & High & High & Medium & 30 & 35 & 35 \\
\hline Permanent & Low & Low & Medium & High & High & High & 20 & 35 & 45 \\
\hline Permanent & Low & Low & High & Low & Low & Low & 30 & 35 & 35 \\
\hline Permanent & Low & Low & High & Low & Low & Medium & 20 & 25 & 55 \\
\hline Permanent & Low & Low & High & Low & Low & High & 10 & 25 & 65 \\
\hline Permanent & Low & Low & High & Low & Medium & Low & 35 & 40 & 25 \\
\hline Permanent & Low & Low & High & Low & Medium & Medium & 25 & 30 & 45 \\
\hline Permanent & Low & Low & High & Low & Medium & High & 15 & 30 & 55 \\
\hline Permanent & Low & Low & High & Low & High & Low & 45 & 50 & 5 \\
\hline Permanent & Low & Low & High & Low & High & Medium & 35 & 40 & 25 \\
\hline Permanent & Low & Low & High & Low & High & High & 25 & 40 & 35 \\
\hline Permanent & Low & Low & High & Medium & Low & Low & 25 & 30 & 45 \\
\hline Permanent & Low & Low & High & Medium & Low & Medium & 15 & 20 & 65 \\
\hline Permanent & Low & Low & High & Medium & Low & High & 5 & 20 & 75 \\
\hline Permanent & Low & Low & High & Medium & Medium & Low & 30 & 35 & 35 \\
\hline Permanent & Low & Low & High & Medium & Medium & Medium & 20 & 25 & 55 \\
\hline Permanent & Low & Low & High & Medium & Medium & High & 10 & 25 & 65 \\
\hline Permanent & Low & Low & High & Medium & High & Low & 40 & 45 & 15 \\
\hline Permanent & Low & Low & High & Medium & High & Medium & 30 & 35 & 35 \\
\hline Permanent & Low & Low & High & Medium & High & High & 20 & 35 & 45 \\
\hline Permanent & Low & Low & High & High & Low & Low & 25 & 25 & 50 \\
\hline Permanent & Low & Low & High & High & Low & Medium & 15 & 15 & 70 \\
\hline Permanent & Low & Low & High & High & Low & High & 5 & 15 & 80 \\
\hline Permanent & Low & Low & High & High & Medium & Low & 30 & 30 & 40 \\
\hline Permanent & Low & Low & High & High & Medium & Medium & 20 & 20 & 60 \\
\hline Permanent & Low & Low & High & High & Medium & High & 10 & 20 & 70 \\
\hline
\end{tabular}




\begin{tabular}{|c|c|c|c|c|c|c|c|c|c|}
\hline \multicolumn{7}{|c|}{ Inputs } & \multicolumn{3}{|c|}{ Habitat quality } \\
\hline $\begin{array}{c}\text { Water } \\
\text { availability }\end{array}$ & Emergent & Floating & Terrestrial & $\begin{array}{l}\text { Winter } \\
\text { refuge }\end{array}$ & Submerged & $\begin{array}{l}\text { Water } \\
\text { quality }\end{array}$ & Low & Average & High \\
\hline Permanent & Low & Low & High & High & High & Low & 40 & 40 & 20 \\
\hline Permanent & Low & Low & High & High & High & Medium & 30 & 30 & 40 \\
\hline Permanent & Low & Low & High & High & High & High & 20 & 30 & 50 \\
\hline Permanent & Low & Medium & Low & Low & Low & Low & 40 & 45 & 15 \\
\hline Permanent & Low & Medium & Low & Low & Low & Medium & 30 & 35 & 35 \\
\hline Permanent & Low & Medium & Low & Low & Low & High & 20 & 35 & 45 \\
\hline Permanent & Low & Medium & Low & Low & Medium & Low & 45 & 50 & 5 \\
\hline Permanent & Low & Medium & Low & Low & Medium & Medium & 35 & 40 & 25 \\
\hline Permanent & Low & Medium & Low & Low & Medium & High & 25 & 40 & 35 \\
\hline Permanent & Low & Medium & Low & Low & High & Low & 50 & 50 & 0 \\
\hline Permanent & Low & Medium & Low & Low & High & Medium & 45 & 50 & 5 \\
\hline Permanent & Low & Medium & Low & Low & High & High & 35 & 50 & 15 \\
\hline Permanent & Low & Medium & Low & Medium & Low & Low & 35 & 40 & 25 \\
\hline Permanent & Low & Medium & Low & Medium & Low & Medium & 25 & 30 & 45 \\
\hline Permanent & Low & Medium & Low & Medium & Low & High & 15 & 30 & 55 \\
\hline Permanent & Low & Medium & Low & Medium & Medium & Low & 40 & 45 & 15 \\
\hline Permanent & Low & Medium & Low & Medium & Medium & Medium & 30 & 35 & 35 \\
\hline Permanent & Low & Medium & Low & Medium & Medium & High & 20 & 35 & 45 \\
\hline Permanent & Low & Medium & Low & Medium & High & Low & 50 & 50 & 0 \\
\hline Permanent & Low & Medium & Low & Medium & High & Medium & 40 & 45 & 15 \\
\hline Permanent & Low & Medium & Low & Medium & High & High & 30 & 45 & 25 \\
\hline Permanent & Low & Medium & Low & High & Low & Low & 35 & 35 & 30 \\
\hline Permanent & Low & Medium & Low & High & Low & Medium & 25 & 25 & 50 \\
\hline Permanent & Low & Medium & Low & High & Low & High & 15 & 25 & 60 \\
\hline Permanent & Low & Medium & Low & High & Medium & Low & 40 & 40 & 20 \\
\hline Permanent & Low & Medium & Low & High & Medium & Medium & 30 & 30 & 40 \\
\hline Permanent & Low & Medium & Low & High & Medium & High & 20 & 30 & 50 \\
\hline Permanent & Low & Medium & Low & High & High & Low & 50 & 50 & 0 \\
\hline Permanent & Low & Medium & Low & High & High & Medium & 40 & 40 & 20 \\
\hline Permanent & Low & Medium & Low & High & High & High & 30 & 40 & 30 \\
\hline Permanent & Low & Medium & Medium & Low & Low & Low & 35 & 40 & 25 \\
\hline Permanent & Low & Medium & Medium & Low & Low & Medium & 25 & 30 & 45 \\
\hline Permanent & Low & Medium & Medium & Low & Low & High & 15 & 30 & 55 \\
\hline Permanent & Low & Medium & Medium & Low & Medium & Low & 40 & 45 & 15 \\
\hline Permanent & Low & Medium & Medium & Low & Medium & Medium & 30 & 35 & 35 \\
\hline Permanent & Low & Medium & Medium & Low & Medium & High & 20 & 35 & 45 \\
\hline Permanent & Low & Medium & Medium & Low & High & Low & 50 & 50 & 0 \\
\hline Permanent & Low & Medium & Medium & Low & High & Medium & 40 & 45 & 15 \\
\hline Permanent & Low & Medium & Medium & Low & High & High & 30 & 45 & 25 \\
\hline Permanent & Low & Medium & Medium & Medium & Low & Low & 30 & 35 & 35 \\
\hline
\end{tabular}




\begin{tabular}{|c|c|c|c|c|c|c|c|c|c|}
\hline \multicolumn{7}{|c|}{ Inputs } & \multicolumn{3}{|c|}{ Habitat quality } \\
\hline $\begin{array}{c}\text { Water } \\
\text { availability }\end{array}$ & Emergent & Floating & Terrestrial & $\begin{array}{l}\text { Winter } \\
\text { refuge }\end{array}$ & Submerged & $\begin{array}{l}\text { Water } \\
\text { quality }\end{array}$ & Low & Average & High \\
\hline Permanent & Low & Medium & Medium & Medium & Low & Medium & 20 & 25 & 55 \\
\hline Permanent & Low & Medium & Medium & Medium & Low & High & 10 & 25 & 65 \\
\hline Permanent & Low & Medium & Medium & Medium & Medium & Low & 35 & 40 & 25 \\
\hline Permanent & Low & Medium & Medium & Medium & Medium & Medium & 25 & 30 & 45 \\
\hline Permanent & Low & Medium & Medium & Medium & Medium & High & 15 & 30 & 55 \\
\hline Permanent & Low & Medium & Medium & Medium & High & Low & 45 & 50 & 5 \\
\hline Permanent & Low & Medium & Medium & Medium & High & Medium & 35 & 40 & 25 \\
\hline Permanent & Low & Medium & Medium & Medium & High & High & 25 & 40 & 35 \\
\hline Permanent & Low & Medium & Medium & High & Low & Low & 30 & 30 & 40 \\
\hline Permanent & Low & Medium & Medium & High & Low & Medium & 20 & 20 & 60 \\
\hline Permanent & Low & Medium & Medium & High & Low & High & 10 & 20 & 70 \\
\hline Permanent & Low & Medium & Medium & High & Medium & Low & 35 & 35 & 30 \\
\hline Permanent & Low & Medium & Medium & High & Medium & Medium & 25 & 25 & 50 \\
\hline Permanent & Low & Medium & Medium & High & Medium & High & 15 & 25 & 60 \\
\hline Permanent & Low & Medium & Medium & High & High & Low & 45 & 45 & 10 \\
\hline Permanent & Low & Medium & Medium & High & High & Medium & 35 & 35 & 30 \\
\hline Permanent & Low & Medium & Medium & High & High & High & 25 & 35 & 40 \\
\hline Permanent & Low & Medium & High & Low & Low & Low & 35 & 35 & 30 \\
\hline Permanent & Low & Medium & High & Low & Low & Medium & 25 & 25 & 50 \\
\hline Permanent & Low & Medium & High & Low & Low & High & 15 & 25 & 60 \\
\hline Permanent & Low & Medium & High & Low & Medium & Low & 40 & 40 & 20 \\
\hline Permanent & Low & Medium & High & Low & Medium & Medium & 30 & 30 & 40 \\
\hline Permanent & Low & Medium & High & Low & Medium & High & 20 & 30 & 50 \\
\hline Permanent & Low & Medium & High & Low & High & Low & 50 & 50 & 0 \\
\hline Permanent & Low & Medium & High & Low & High & Medium & 40 & 40 & 20 \\
\hline Permanent & Low & Medium & High & Low & High & High & 30 & 40 & 30 \\
\hline Permanent & Low & Medium & High & Medium & Low & Low & 30 & 30 & 40 \\
\hline Permanent & Low & Medium & High & Medium & Low & Medium & 20 & 20 & 60 \\
\hline Permanent & Low & Medium & High & Medium & Low & High & 10 & 20 & 70 \\
\hline Permanent & Low & Medium & High & Medium & Medium & Low & 35 & 35 & 30 \\
\hline Permanent & Low & Medium & High & Medium & Medium & Medium & 25 & 25 & 50 \\
\hline Permanent & Low & Medium & High & Medium & Medium & High & 15 & 25 & 60 \\
\hline Permanent & Low & Medium & High & Medium & High & Low & 45 & 45 & 10 \\
\hline Permanent & Low & Medium & High & Medium & High & Medium & 35 & 35 & 30 \\
\hline Permanent & Low & Medium & High & Medium & High & High & 25 & 35 & 40 \\
\hline Permanent & Low & Medium & High & High & Low & Low & 30 & 25 & 45 \\
\hline Permanent & Low & Medium & High & High & Low & Medium & 20 & 15 & 65 \\
\hline Permanent & Low & Medium & High & High & Low & High & 10 & 15 & 75 \\
\hline Permanent & Low & Medium & High & High & Medium & Low & 35 & 30 & 35 \\
\hline Permanent & Low & Medium & High & High & Medium & Medium & 25 & 20 & 55 \\
\hline
\end{tabular}




\begin{tabular}{|c|c|c|c|c|c|c|c|c|c|}
\hline \multicolumn{7}{|c|}{ Inputs } & \multicolumn{3}{|c|}{ Habitat quality } \\
\hline $\begin{array}{c}\text { Water } \\
\text { availability }\end{array}$ & Emergent & Floating & Terrestrial & $\begin{array}{l}\text { Winter } \\
\text { refuge }\end{array}$ & Submerged & $\begin{array}{l}\text { Water } \\
\text { quality }\end{array}$ & Low & Average & High \\
\hline Permanent & Low & Medium & High & High & Medium & High & 15 & 20 & 65 \\
\hline Permanent & Low & Medium & High & High & High & Low & 45 & 40 & 15 \\
\hline Permanent & Low & Medium & High & High & High & Medium & 35 & 30 & 35 \\
\hline Permanent & Low & Medium & High & High & High & High & 25 & 30 & 45 \\
\hline Permanent & Low & High & Low & Low & Low & Low & 45 & 50 & 5 \\
\hline Permanent & Low & High & Low & Low & Low & Medium & 35 & 40 & 25 \\
\hline Permanent & Low & High & Low & Low & Low & High & 25 & 40 & 35 \\
\hline Permanent & Low & High & Low & Low & Medium & Low & 50 & 50 & 0 \\
\hline Permanent & Low & High & Low & Low & Medium & Medium & 40 & 45 & 15 \\
\hline Permanent & Low & High & Low & Low & Medium & High & 30 & 45 & 25 \\
\hline Permanent & Low & High & Low & Low & High & Low & 55 & 45 & 0 \\
\hline Permanent & Low & High & Low & Low & High & Medium & 50 & 50 & 0 \\
\hline Permanent & Low & High & Low & Low & High & High & 40 & 55 & 5 \\
\hline Permanent & Low & High & Low & Medium & Low & Low & 40 & 45 & 15 \\
\hline Permanent & Low & High & Low & Medium & Low & Medium & 30 & 35 & 35 \\
\hline Permanent & Low & High & Low & Medium & Low & High & 20 & 35 & 45 \\
\hline Permanent & Low & High & Low & Medium & Medium & Low & 45 & 50 & 5 \\
\hline Permanent & Low & High & Low & Medium & Medium & Medium & 35 & 40 & 25 \\
\hline Permanent & Low & High & Low & Medium & Medium & High & 25 & 40 & 35 \\
\hline Permanent & Low & High & Low & Medium & High & Low & 50 & 50 & 0 \\
\hline Permanent & Low & High & Low & Medium & High & Medium & 45 & 50 & 5 \\
\hline Permanent & Low & High & Low & Medium & High & High & 35 & 50 & 15 \\
\hline Permanent & Low & High & Low & High & Low & Low & 40 & 40 & 20 \\
\hline Permanent & Low & High & Low & High & Low & Medium & 30 & 30 & 40 \\
\hline Permanent & Low & High & Low & High & Low & High & 20 & 30 & 50 \\
\hline Permanent & Low & High & Low & High & Medium & Low & 45 & 45 & 10 \\
\hline Permanent & Low & High & Low & High & Medium & Medium & 35 & 35 & 30 \\
\hline Permanent & Low & High & Low & High & Medium & High & 25 & 35 & 40 \\
\hline Permanent & Low & High & Low & High & High & Low & 50 & 50 & 0 \\
\hline Permanent & Low & High & Low & High & High & Medium & 45 & 45 & 10 \\
\hline Permanent & Low & High & Low & High & High & High & 35 & 45 & 20 \\
\hline Permanent & Low & High & Medium & Low & Low & Low & 40 & 45 & 15 \\
\hline Permanent & Low & High & Medium & Low & Low & Medium & 30 & 35 & 35 \\
\hline Permanent & Low & High & Medium & Low & Low & High & 20 & 35 & 45 \\
\hline Permanent & Low & High & Medium & Low & Medium & Low & 45 & 50 & 5 \\
\hline Permanent & Low & High & Medium & Low & Medium & Medium & 35 & 40 & 25 \\
\hline Permanent & Low & High & Medium & Low & Medium & High & 25 & 40 & 35 \\
\hline Permanent & Low & High & Medium & Low & High & Low & 50 & 50 & 0 \\
\hline Permanent & Low & High & Medium & Low & High & Medium & 45 & 50 & 5 \\
\hline Permanent & Low & High & Medium & Low & High & High & 35 & 50 & 15 \\
\hline
\end{tabular}




\begin{tabular}{|c|c|c|c|c|c|c|c|c|c|}
\hline \multicolumn{7}{|c|}{ Inputs } & \multicolumn{3}{|c|}{ Habitat quality } \\
\hline $\begin{array}{c}\text { Water } \\
\text { availability }\end{array}$ & Emergent & Floating & Terrestrial & $\begin{array}{l}\text { Winter } \\
\text { refuge }\end{array}$ & Submerged & $\begin{array}{l}\text { Water } \\
\text { quality }\end{array}$ & Low & Average & High \\
\hline Permanent & Low & High & Medium & Medium & Low & Low & 35 & 40 & 25 \\
\hline Permanent & Low & High & Medium & Medium & Low & Medium & 25 & 30 & 45 \\
\hline Permanent & Low & High & Medium & Medium & Low & High & 15 & 30 & 55 \\
\hline Permanent & Low & High & Medium & Medium & Medium & Low & 40 & 45 & 15 \\
\hline Permanent & Low & High & Medium & Medium & Medium & Medium & 30 & 35 & 35 \\
\hline Permanent & Low & High & Medium & Medium & Medium & High & 20 & 35 & 45 \\
\hline Permanent & Low & High & Medium & Medium & High & Low & 45 & 55 & 0 \\
\hline Permanent & Low & High & Medium & Medium & High & Medium & 40 & 45 & 15 \\
\hline Permanent & Low & High & Medium & Medium & High & High & 30 & 45 & 25 \\
\hline Permanent & Low & High & Medium & High & Low & Low & 35 & 35 & 30 \\
\hline Permanent & Low & High & Medium & High & Low & Medium & 25 & 25 & 50 \\
\hline Permanent & Low & High & Medium & High & Low & High & 15 & 25 & 60 \\
\hline Permanent & Low & High & Medium & High & Medium & Low & 40 & 40 & 20 \\
\hline Permanent & Low & High & Medium & High & Medium & Medium & 30 & 30 & 40 \\
\hline Permanent & Low & High & Medium & High & Medium & High & 20 & 30 & 50 \\
\hline Permanent & Low & High & Medium & High & High & Low & 50 & 50 & 0 \\
\hline Permanent & Low & High & Medium & High & High & Medium & 40 & 40 & 20 \\
\hline Permanent & Low & High & Medium & High & High & High & 30 & 40 & 30 \\
\hline Permanent & Low & High & High & Low & Low & Low & 40 & 40 & 20 \\
\hline Permanent & Low & High & High & Low & Low & Medium & 30 & 30 & 40 \\
\hline Permanent & Low & High & High & Low & Low & High & 20 & 30 & 50 \\
\hline Permanent & Low & High & High & Low & Medium & Low & 45 & 45 & 10 \\
\hline Permanent & Low & High & High & Low & Medium & Medium & 35 & 35 & 30 \\
\hline Permanent & Low & High & High & Low & Medium & High & 25 & 35 & 40 \\
\hline Permanent & Low & High & High & Low & High & Low & 50 & 50 & 0 \\
\hline Permanent & Low & High & High & Low & High & Medium & 45 & 45 & 10 \\
\hline Permanent & Low & High & High & Low & High & High & 35 & 45 & 20 \\
\hline Permanent & Low & High & High & Medium & Low & Low & 35 & 35 & 30 \\
\hline Permanent & Low & High & High & Medium & Low & Medium & 25 & 25 & 50 \\
\hline Permanent & Low & High & High & Medium & Low & High & 15 & 25 & 60 \\
\hline Permanent & Low & High & High & Medium & Medium & Low & 40 & 40 & 20 \\
\hline Permanent & Low & High & High & Medium & Medium & Medium & 30 & 30 & 40 \\
\hline Permanent & Low & High & High & Medium & Medium & High & 20 & 30 & 50 \\
\hline Permanent & Low & High & High & Medium & High & Low & 50 & 50 & 0 \\
\hline Permanent & Low & High & High & Medium & High & Medium & 40 & 40 & 20 \\
\hline Permanent & Low & High & High & Medium & High & High & 30 & 40 & 30 \\
\hline Permanent & Low & High & High & High & Low & Low & 35 & 30 & 35 \\
\hline Permanent & Low & High & High & High & Low & Medium & 25 & 20 & 55 \\
\hline Permanent & Low & High & High & High & Low & High & 15 & 20 & 65 \\
\hline Permanent & Low & High & High & High & Medium & Low & 40 & 35 & 25 \\
\hline
\end{tabular}




\begin{tabular}{|c|c|c|c|c|c|c|c|c|c|}
\hline \multicolumn{7}{|c|}{ Inputs } & \multicolumn{3}{|c|}{ Habitat quality } \\
\hline $\begin{array}{c}\text { Water } \\
\text { availability }\end{array}$ & Emergent & Floating & Terrestrial & $\begin{array}{l}\text { Winter } \\
\text { refuge }\end{array}$ & Submerged & $\begin{array}{l}\text { Water } \\
\text { quality }\end{array}$ & Low & Average & High \\
\hline Permanent & Low & High & High & High & Medium & Medium & 30 & 25 & 45 \\
\hline Permanent & Low & High & High & High & Medium & High & 20 & 25 & 55 \\
\hline Permanent & Low & High & High & High & High & Low & 50 & 45 & 5 \\
\hline Permanent & Low & High & High & High & High & Medium & 40 & 35 & 25 \\
\hline Permanent & Low & High & High & High & High & High & 30 & 35 & 35 \\
\hline Permanent & Medium & Low & Low & Low & Low & Low & 30 & 40 & 30 \\
\hline Permanent & Medium & Low & Low & Low & Low & Medium & 20 & 30 & 50 \\
\hline Permanent & Medium & Low & Low & Low & Low & High & 10 & 30 & 60 \\
\hline Permanent & Medium & Low & Low & Low & Medium & Low & 35 & 45 & 20 \\
\hline Permanent & Medium & Low & Low & Low & Medium & Medium & 25 & 35 & 40 \\
\hline Permanent & Medium & Low & Low & Low & Medium & High & 15 & 35 & 50 \\
\hline Permanent & Medium & Low & Low & Low & High & Low & 45 & 55 & 0 \\
\hline Permanent & Medium & Low & Low & Low & High & Medium & 35 & 45 & 20 \\
\hline Permanent & Medium & Low & Low & Low & High & High & 25 & 45 & 30 \\
\hline Permanent & Medium & Low & Low & Medium & Low & Low & 25 & 35 & 40 \\
\hline Permanent & Medium & Low & Low & Medium & Low & Medium & 15 & 25 & 60 \\
\hline Permanent & Medium & Low & Low & Medium & Low & High & 5 & 25 & 70 \\
\hline Permanent & Medium & Low & Low & Medium & Medium & Low & 30 & 40 & 30 \\
\hline Permanent & Medium & Low & Low & Medium & Medium & Medium & 20 & 30 & 50 \\
\hline Permanent & Medium & Low & Low & Medium & Medium & High & 10 & 30 & 60 \\
\hline Permanent & Medium & Low & Low & Medium & High & Low & 40 & 50 & 10 \\
\hline Permanent & Medium & Low & Low & Medium & High & Medium & 30 & 40 & 30 \\
\hline Permanent & Medium & Low & Low & Medium & High & High & 20 & 40 & 40 \\
\hline Permanent & Medium & Low & Low & High & Low & Low & 25 & 30 & 45 \\
\hline Permanent & Medium & Low & Low & High & Low & Medium & 15 & 20 & 65 \\
\hline Permanent & Medium & Low & Low & High & Low & High & 5 & 20 & 75 \\
\hline Permanent & Medium & Low & Low & High & Medium & Low & 30 & 35 & 35 \\
\hline Permanent & Medium & Low & Low & High & Medium & Medium & 20 & 25 & 55 \\
\hline Permanent & Medium & Low & Low & High & Medium & High & 10 & 25 & 65 \\
\hline Permanent & Medium & Low & Low & High & High & Low & 40 & 45 & 15 \\
\hline Permanent & Medium & Low & Low & High & High & Medium & 30 & 35 & 35 \\
\hline Permanent & Medium & Low & Low & High & High & High & 20 & 35 & 45 \\
\hline Permanent & Medium & Low & Medium & Low & Low & Low & 25 & 35 & 40 \\
\hline Permanent & Medium & Low & Medium & Low & Low & Medium & 15 & 25 & 60 \\
\hline Permanent & Medium & Low & Medium & Low & Low & High & 5 & 25 & 70 \\
\hline Permanent & Medium & Low & Medium & Low & Medium & Low & 30 & 40 & 30 \\
\hline Permanent & Medium & Low & Medium & Low & Medium & Medium & 20 & 30 & 50 \\
\hline Permanent & Medium & Low & Medium & Low & Medium & High & 10 & 30 & 60 \\
\hline Permanent & Medium & Low & Medium & Low & High & Low & 40 & 50 & 10 \\
\hline Permanent & Medium & Low & Medium & Low & High & Medium & 30 & 40 & 30 \\
\hline
\end{tabular}




\begin{tabular}{|c|c|c|c|c|c|c|c|c|c|}
\hline \multicolumn{7}{|c|}{ Inputs } & \multicolumn{3}{|c|}{ Habitat quality } \\
\hline $\begin{array}{c}\text { Water } \\
\text { availability }\end{array}$ & Emergent & Floating & Terrestrial & $\begin{array}{l}\text { Winter } \\
\text { refuge }\end{array}$ & Submerged & $\begin{array}{l}\text { Water } \\
\text { quality }\end{array}$ & Low & Average & High \\
\hline Permanent & Medium & Low & Medium & Low & High & High & 20 & 40 & 40 \\
\hline Permanent & Medium & Low & Medium & Medium & Low & Low & 20 & 30 & 50 \\
\hline Permanent & Medium & Low & Medium & Medium & Low & Medium & 10 & 20 & 70 \\
\hline Permanent & Medium & Low & Medium & Medium & Low & High & 0 & 20 & 80 \\
\hline Permanent & Medium & Low & Medium & Medium & Medium & Low & 25 & 35 & 40 \\
\hline Permanent & Medium & Low & Medium & Medium & Medium & Medium & 15 & 25 & 60 \\
\hline Permanent & Medium & Low & Medium & Medium & Medium & High & 5 & 25 & 70 \\
\hline Permanent & Medium & Low & Medium & Medium & High & Low & 35 & 45 & 20 \\
\hline Permanent & Medium & Low & Medium & Medium & High & Medium & 25 & 35 & 40 \\
\hline Permanent & Medium & Low & Medium & Medium & High & High & 15 & 35 & 50 \\
\hline Permanent & Medium & Low & Medium & High & Low & Low & 20 & 25 & 55 \\
\hline Permanent & Medium & Low & Medium & High & Low & Medium & 10 & 15 & 75 \\
\hline Permanent & Medium & Low & Medium & High & Low & High & 0 & 15 & 85 \\
\hline Permanent & Medium & Low & Medium & High & Medium & Low & 25 & 30 & 45 \\
\hline Permanent & Medium & Low & Medium & High & Medium & Medium & 15 & 20 & 65 \\
\hline Permanent & Medium & Low & Medium & High & Medium & High & 5 & 20 & 75 \\
\hline Permanent & Medium & Low & Medium & High & High & Low & 35 & 40 & 25 \\
\hline Permanent & Medium & Low & Medium & High & High & Medium & 25 & 30 & 45 \\
\hline Permanent & Medium & Low & Medium & High & High & High & 15 & 30 & 55 \\
\hline Permanent & Medium & Low & High & Low & Low & Low & 25 & 30 & 45 \\
\hline Permanent & Medium & Low & High & Low & Low & Medium & 15 & 20 & 65 \\
\hline Permanent & Medium & Low & High & Low & Low & High & 5 & 20 & 75 \\
\hline Permanent & Medium & Low & High & Low & Medium & Low & 30 & 35 & 35 \\
\hline Permanent & Medium & Low & High & Low & Medium & Medium & 20 & 25 & 55 \\
\hline Permanent & Medium & Low & High & Low & Medium & High & 10 & 25 & 65 \\
\hline Permanent & Medium & Low & High & Low & High & Low & 40 & 45 & 15 \\
\hline Permanent & Medium & Low & High & Low & High & Medium & 30 & 35 & 35 \\
\hline Permanent & Medium & Low & High & Low & High & High & 20 & 35 & 45 \\
\hline Permanent & Medium & Low & High & Medium & Low & Low & 20 & 25 & 55 \\
\hline Permanent & Medium & Low & High & Medium & Low & Medium & 10 & 15 & 75 \\
\hline Permanent & Medium & Low & High & Medium & Low & High & 0 & 15 & 85 \\
\hline Permanent & Medium & Low & High & Medium & Medium & Low & 25 & 30 & 45 \\
\hline Permanent & Medium & Low & High & Medium & Medium & Medium & 15 & 20 & 65 \\
\hline Permanent & Medium & Low & High & Medium & Medium & High & 5 & 20 & 75 \\
\hline Permanent & Medium & Low & High & Medium & High & Low & 35 & 40 & 25 \\
\hline Permanent & Medium & Low & High & Medium & High & Medium & 25 & 30 & 45 \\
\hline Permanent & Medium & Low & High & Medium & High & High & 15 & 30 & 55 \\
\hline Permanent & Medium & Low & High & High & Low & Low & 20 & 20 & 60 \\
\hline Permanent & Medium & Low & High & High & Low & Medium & 10 & 10 & 80 \\
\hline Permanent & Medium & Low & High & High & Low & High & 0 & 10 & 90 \\
\hline
\end{tabular}




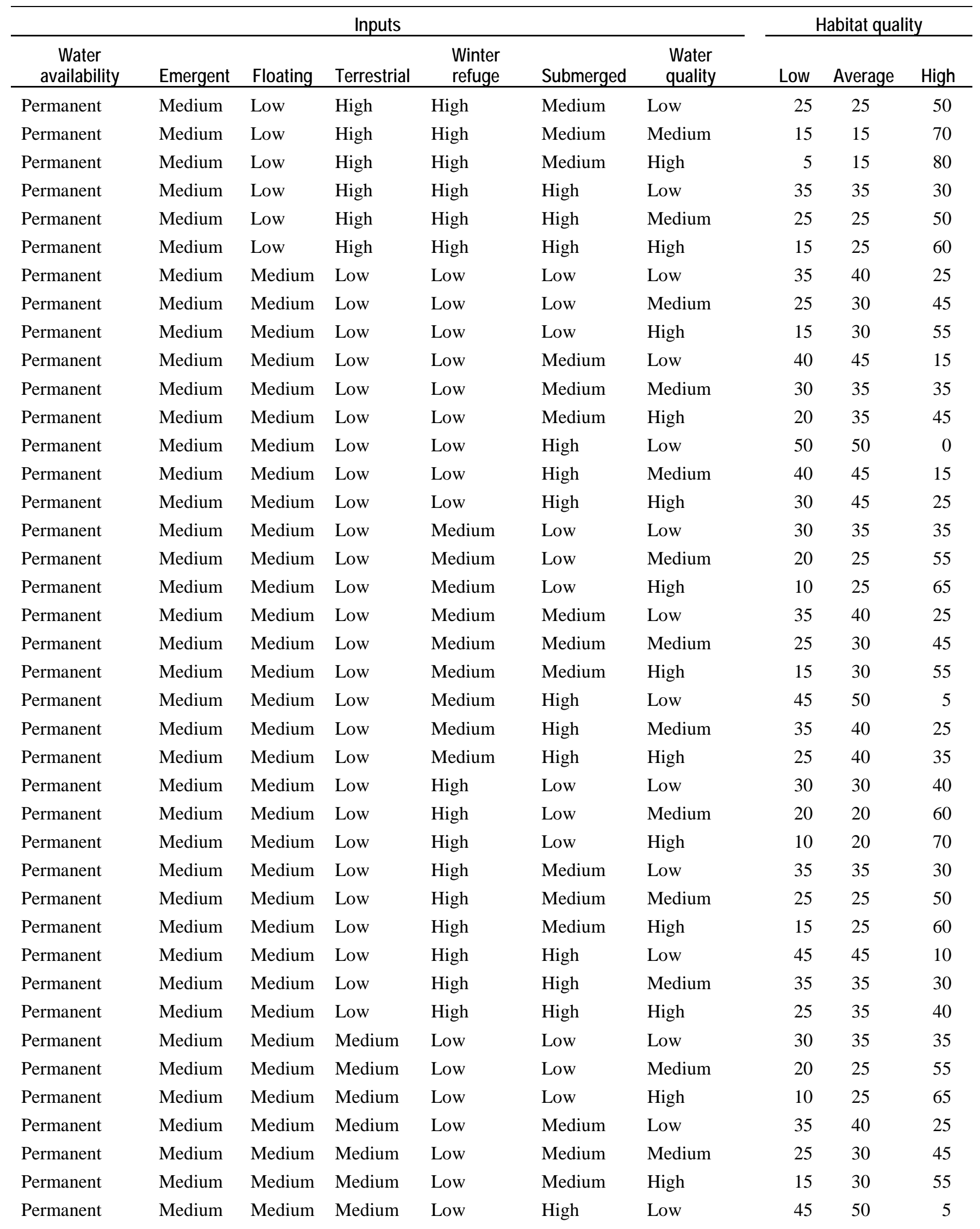




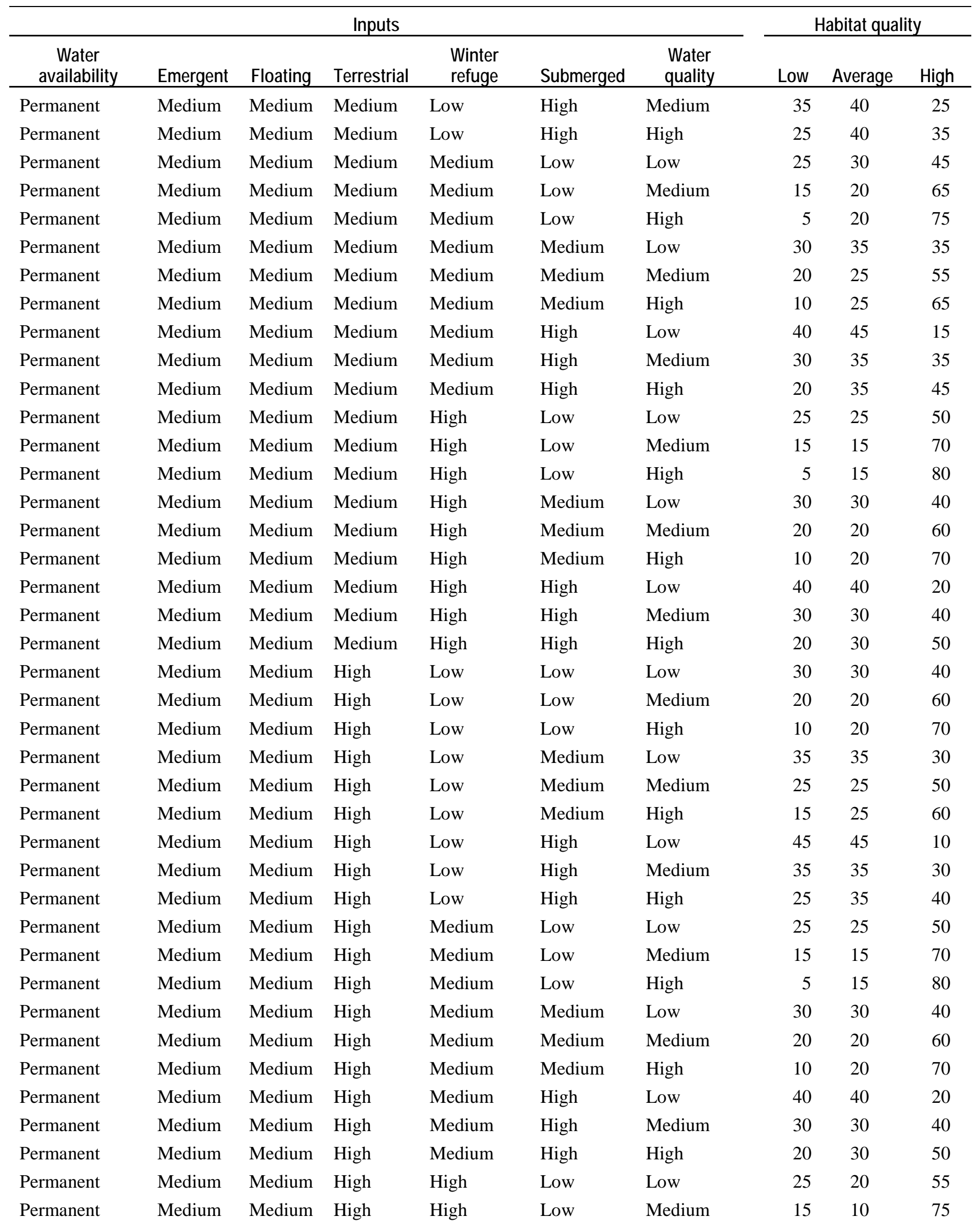




\begin{tabular}{|c|c|c|c|c|c|c|c|c|c|}
\hline \multicolumn{7}{|c|}{ Inputs } & \multicolumn{3}{|c|}{ Habitat quality } \\
\hline $\begin{array}{c}\text { Water } \\
\text { availability }\end{array}$ & Emergent & Floating & Terrestrial & $\begin{array}{l}\text { Winter } \\
\text { refuge }\end{array}$ & Submerged & $\begin{array}{l}\text { Water } \\
\text { quality }\end{array}$ & Low & Average & High \\
\hline Permanent & Medium & Medium & High & High & Low & High & 5 & 10 & 85 \\
\hline Permanent & Medium & Medium & High & High & Medium & Low & 30 & 25 & 45 \\
\hline Permanent & Medium & Medium & High & High & Medium & Medium & 20 & 15 & 65 \\
\hline Permanent & Medium & Medium & High & High & Medium & High & 10 & 15 & 75 \\
\hline Permanent & Medium & Medium & High & High & High & Low & 40 & 35 & 25 \\
\hline Permanent & Medium & Medium & High & High & High & Medium & 30 & 25 & 45 \\
\hline Permanent & Medium & Medium & High & High & High & High & 20 & 25 & 55 \\
\hline Permanent & Medium & High & Low & Low & Low & Low & 40 & 45 & 15 \\
\hline Permanent & Medium & High & Low & Low & Low & Medium & 30 & 35 & 35 \\
\hline Permanent & Medium & High & Low & Low & Low & High & 20 & 35 & 45 \\
\hline Permanent & Medium & High & Low & Low & Medium & Low & 45 & 50 & 5 \\
\hline Permanent & Medium & High & Low & Low & Medium & Medium & 35 & 40 & 25 \\
\hline Permanent & Medium & High & Low & Low & Medium & High & 25 & 40 & 35 \\
\hline Permanent & Medium & High & Low & Low & High & Low & 55 & 45 & 0 \\
\hline Permanent & Medium & High & Low & Low & High & Medium & 45 & 50 & 5 \\
\hline Permanent & Medium & High & Low & Low & High & High & 35 & 50 & 15 \\
\hline Permanent & Medium & High & Low & Medium & Low & Low & 35 & 40 & 25 \\
\hline Permanent & Medium & High & Low & Medium & Low & Medium & 25 & 30 & 45 \\
\hline Permanent & Medium & High & Low & Medium & Low & High & 15 & 30 & 55 \\
\hline Permanent & Medium & High & Low & Medium & Medium & Low & 40 & 45 & 15 \\
\hline Permanent & Medium & High & Low & Medium & Medium & Medium & 30 & 35 & 35 \\
\hline Permanent & Medium & High & Low & Medium & Medium & High & 20 & 35 & 45 \\
\hline Permanent & Medium & High & Low & Medium & High & Low & 50 & 50 & 0 \\
\hline Permanent & Medium & High & Low & Medium & High & Medium & 40 & 45 & 15 \\
\hline Permanent & Medium & High & Low & Medium & High & High & 30 & 45 & 25 \\
\hline Permanent & Medium & High & Low & High & Low & Low & 35 & 35 & 30 \\
\hline Permanent & Medium & High & Low & High & Low & Medium & 25 & 25 & 50 \\
\hline Permanent & Medium & High & Low & High & Low & High & 15 & 25 & 60 \\
\hline Permanent & Medium & High & Low & High & Medium & Low & 40 & 40 & 20 \\
\hline Permanent & Medium & High & Low & High & Medium & Medium & 30 & 30 & 40 \\
\hline Permanent & Medium & High & Low & High & Medium & High & 20 & 30 & 50 \\
\hline Permanent & Medium & High & Low & High & High & Low & 50 & 50 & 0 \\
\hline Permanent & Medium & High & Low & High & High & Medium & 40 & 40 & 20 \\
\hline Permanent & Medium & High & Low & High & High & High & 30 & 40 & 30 \\
\hline Permanent & Medium & High & Medium & Low & Low & Low & 35 & 40 & 25 \\
\hline Permanent & Medium & High & Medium & Low & Low & Medium & 25 & 30 & 45 \\
\hline Permanent & Medium & High & Medium & Low & Low & High & 15 & 30 & 55 \\
\hline Permanent & Medium & High & Medium & Low & Medium & Low & 40 & 45 & 15 \\
\hline Permanent & Medium & High & Medium & Low & Medium & Medium & 30 & 35 & 35 \\
\hline Permanent & Medium & High & Medium & Low & Medium & High & 20 & 35 & 45 \\
\hline
\end{tabular}




\begin{tabular}{|c|c|c|c|c|c|c|c|c|c|}
\hline \multicolumn{7}{|c|}{ Inputs } & \multicolumn{3}{|c|}{ Habitat quality } \\
\hline $\begin{array}{c}\text { Water } \\
\text { availability }\end{array}$ & Emergent & Floating & Terrestrial & $\begin{array}{l}\text { Winter } \\
\text { refuge }\end{array}$ & Submerged & $\begin{array}{l}\text { Water } \\
\text { quality }\end{array}$ & Low & Average & High \\
\hline Permanent & Medium & High & Medium & Low & High & Low & 50 & 50 & 0 \\
\hline Permanent & Medium & High & Medium & Low & High & Medium & 40 & 45 & 15 \\
\hline Permanent & Medium & High & Medium & Low & High & High & 30 & 45 & 25 \\
\hline Permanent & Medium & High & Medium & Medium & Low & Low & 30 & 35 & 35 \\
\hline Permanent & Medium & High & Medium & Medium & Low & Medium & 20 & 25 & 55 \\
\hline Permanent & Medium & High & Medium & Medium & Low & High & 10 & 25 & 65 \\
\hline Permanent & Medium & High & Medium & Medium & Medium & Low & 35 & 40 & 25 \\
\hline Permanent & Medium & High & Medium & Medium & Medium & Medium & 25 & 30 & 45 \\
\hline Permanent & Medium & High & Medium & Medium & Medium & High & 15 & 30 & 55 \\
\hline Permanent & Medium & High & Medium & Medium & High & Low & 45 & 50 & 5 \\
\hline Permanent & Medium & High & Medium & Medium & High & Medium & 35 & 40 & 25 \\
\hline Permanent & Medium & High & Medium & Medium & High & High & 25 & 40 & 35 \\
\hline Permanent & Medium & High & Medium & High & Low & Low & 30 & 30 & 40 \\
\hline Permanent & Medium & High & Medium & High & Low & Medium & 20 & 20 & 60 \\
\hline Permanent & Medium & High & Medium & High & Low & High & 10 & 20 & 70 \\
\hline Permanent & Medium & High & Medium & High & Medium & Low & 35 & 35 & 30 \\
\hline Permanent & Medium & High & Medium & High & Medium & Medium & 25 & 25 & 50 \\
\hline Permanent & Medium & High & Medium & High & Medium & High & 15 & 25 & 60 \\
\hline Permanent & Medium & High & Medium & High & High & Low & 45 & 45 & 10 \\
\hline Permanent & Medium & High & Medium & High & High & Medium & 35 & 35 & 30 \\
\hline Permanent & Medium & High & Medium & High & High & High & 25 & 35 & 40 \\
\hline Permanent & Medium & High & High & Low & Low & Low & 35 & 35 & 30 \\
\hline Permanent & Medium & High & High & Low & Low & Medium & 25 & 25 & 50 \\
\hline Permanent & Medium & High & High & Low & Low & High & 15 & 25 & 60 \\
\hline Permanent & Medium & High & High & Low & Medium & Low & 40 & 40 & 20 \\
\hline Permanent & Medium & High & High & Low & Medium & Medium & 30 & 30 & 40 \\
\hline Permanent & Medium & High & High & Low & Medium & High & 20 & 30 & 50 \\
\hline Permanent & Medium & High & High & Low & High & Low & 50 & 50 & 0 \\
\hline Permanent & Medium & High & High & Low & High & Medium & 40 & 40 & 20 \\
\hline Permanent & Medium & High & High & Low & High & High & 30 & 40 & 30 \\
\hline Permanent & Medium & High & High & Medium & Low & Low & 30 & 30 & 40 \\
\hline Permanent & Medium & High & High & Medium & Low & Medium & 20 & 20 & 60 \\
\hline Permanent & Medium & High & High & Medium & Low & High & 10 & 20 & 70 \\
\hline Permanent & Medium & High & High & Medium & Medium & Low & 35 & 35 & 30 \\
\hline Permanent & Medium & High & High & Medium & Medium & Medium & 25 & 25 & 50 \\
\hline Permanent & Medium & High & High & Medium & Medium & High & 15 & 25 & 60 \\
\hline Permanent & Medium & High & High & Medium & High & Low & 45 & 45 & 10 \\
\hline Permanent & Medium & High & High & Medium & High & Medium & 35 & 35 & 30 \\
\hline Permanent & Medium & High & High & Medium & High & High & 25 & 35 & 40 \\
\hline Permanent & Medium & High & High & High & Low & Low & 30 & 25 & 45 \\
\hline
\end{tabular}




\begin{tabular}{|c|c|c|c|c|c|c|c|c|c|}
\hline \multicolumn{7}{|c|}{ Inputs } & \multicolumn{3}{|c|}{ Habitat quality } \\
\hline $\begin{array}{c}\text { Water } \\
\text { availability }\end{array}$ & Emergent & Floating & Terrestrial & $\begin{array}{l}\text { Winter } \\
\text { refuge }\end{array}$ & Submerged & $\begin{array}{l}\text { Water } \\
\text { quality }\end{array}$ & Low & Average & High \\
\hline Permanent & Medium & High & High & High & Low & Medium & 20 & 15 & 65 \\
\hline Permanent & Medium & High & High & High & Low & High & 10 & 15 & 75 \\
\hline Permanent & Medium & High & High & High & Medium & Low & 35 & 30 & 35 \\
\hline Permanent & Medium & High & High & High & Medium & Medium & 25 & 20 & 55 \\
\hline Permanent & Medium & High & High & High & Medium & High & 15 & 20 & 65 \\
\hline Permanent & Medium & High & High & High & High & Low & 45 & 40 & 15 \\
\hline Permanent & Medium & High & High & High & High & Medium & 35 & 30 & 35 \\
\hline Permanent & Medium & High & High & High & High & High & 25 & 30 & 45 \\
\hline Permanent & High & Low & Low & Low & Low & Low & 30 & 45 & 25 \\
\hline Permanent & High & Low & Low & Low & Low & Medium & 20 & 35 & 45 \\
\hline Permanent & High & Low & Low & Low & Low & High & 10 & 35 & 55 \\
\hline Permanent & High & Low & Low & Low & Medium & Low & 35 & 50 & 15 \\
\hline Permanent & High & Low & Low & Low & Medium & Medium & 25 & 40 & 35 \\
\hline Permanent & High & Low & Low & Low & Medium & High & 15 & 40 & 45 \\
\hline Permanent & High & Low & Low & Low & High & Low & 45 & 55 & 0 \\
\hline Permanent & High & Low & Low & Low & High & Medium & 35 & 50 & 15 \\
\hline Permanent & High & Low & Low & Low & High & High & 25 & 50 & 25 \\
\hline Permanent & High & Low & Low & Medium & Low & Low & 25 & 40 & 35 \\
\hline Permanent & High & Low & Low & Medium & Low & Medium & 15 & 30 & 55 \\
\hline Permanent & High & Low & Low & Medium & Low & High & 5 & 30 & 65 \\
\hline Permanent & High & Low & Low & Medium & Medium & Low & 30 & 45 & 25 \\
\hline Permanent & High & Low & Low & Medium & Medium & Medium & 20 & 35 & 45 \\
\hline Permanent & High & Low & Low & Medium & Medium & High & 10 & 35 & 55 \\
\hline Permanent & High & Low & Low & Medium & High & Low & 40 & 55 & 5 \\
\hline Permanent & High & Low & Low & Medium & High & Medium & 30 & 45 & 25 \\
\hline Permanent & High & Low & Low & Medium & High & High & 20 & 45 & 35 \\
\hline Permanent & High & Low & Low & High & Low & Low & 25 & 35 & 40 \\
\hline Permanent & High & Low & Low & High & Low & Medium & 15 & 25 & 60 \\
\hline Permanent & High & Low & Low & High & Low & High & 5 & 25 & 70 \\
\hline Permanent & High & Low & Low & High & Medium & Low & 30 & 40 & 30 \\
\hline Permanent & High & Low & Low & High & Medium & Medium & 20 & 30 & 50 \\
\hline Permanent & High & Low & Low & High & Medium & High & 10 & 30 & 60 \\
\hline Permanent & High & Low & Low & High & High & Low & 40 & 50 & 10 \\
\hline Permanent & High & Low & Low & High & High & Medium & 30 & 40 & 30 \\
\hline Permanent & High & Low & Low & High & High & High & 20 & 40 & 40 \\
\hline Permanent & High & Low & Medium & Low & Low & Low & 25 & 40 & 35 \\
\hline Permanent & High & Low & Medium & Low & Low & Medium & 15 & 30 & 55 \\
\hline Permanent & High & Low & Medium & Low & Low & High & 5 & 30 & 65 \\
\hline Permanent & High & Low & Medium & Low & Medium & Low & 30 & 45 & 25 \\
\hline Permanent & High & Low & Medium & Low & Medium & Medium & 20 & 35 & 45 \\
\hline
\end{tabular}




\begin{tabular}{|c|c|c|c|c|c|c|c|c|c|}
\hline \multicolumn{7}{|c|}{ Inputs } & \multicolumn{3}{|c|}{ Habitat quality } \\
\hline $\begin{array}{c}\text { Water } \\
\text { availability }\end{array}$ & Emergent & Floating & Terrestrial & $\begin{array}{l}\text { Winter } \\
\text { refuge }\end{array}$ & Submerged & $\begin{array}{l}\text { Water } \\
\text { quality }\end{array}$ & Low & Average & High \\
\hline Permanent & High & Low & Medium & Low & Medium & High & 10 & 35 & 55 \\
\hline Permanent & High & Low & Medium & Low & High & Low & 40 & 55 & 5 \\
\hline Permanent & High & Low & Medium & Low & High & Medium & 30 & 45 & 25 \\
\hline Permanent & High & Low & Medium & Low & High & High & 20 & 45 & 35 \\
\hline Permanent & High & Low & Medium & Medium & Low & Low & 20 & 35 & 45 \\
\hline Permanent & High & Low & Medium & Medium & Low & Medium & 10 & 25 & 65 \\
\hline Permanent & High & Low & Medium & Medium & Low & High & 0 & 25 & 75 \\
\hline Permanent & High & Low & Medium & Medium & Medium & Low & 25 & 40 & 35 \\
\hline Permanent & High & Low & Medium & Medium & Medium & Medium & 15 & 30 & 55 \\
\hline Permanent & High & Low & Medium & Medium & Medium & High & 5 & 30 & 65 \\
\hline Permanent & High & Low & Medium & Medium & High & Low & 35 & 50 & 15 \\
\hline Permanent & High & Low & Medium & Medium & High & Medium & 25 & 40 & 35 \\
\hline Permanent & High & Low & Medium & Medium & High & High & 15 & 40 & 45 \\
\hline Permanent & High & Low & Medium & High & Low & Low & 20 & 30 & 50 \\
\hline Permanent & High & Low & Medium & High & Low & Medium & 10 & 20 & 70 \\
\hline Permanent & High & Low & Medium & High & Low & High & 0 & 20 & 80 \\
\hline Permanent & High & Low & Medium & High & Medium & Low & 25 & 35 & 40 \\
\hline Permanent & High & Low & Medium & High & Medium & Medium & 15 & 25 & 60 \\
\hline Permanent & High & Low & Medium & High & Medium & High & 5 & 25 & 70 \\
\hline Permanent & High & Low & Medium & High & High & Low & 35 & 45 & 20 \\
\hline Permanent & High & Low & Medium & High & High & Medium & 25 & 35 & 40 \\
\hline Permanent & High & Low & Medium & High & High & High & 15 & 35 & 50 \\
\hline Permanent & High & Low & High & Low & Low & Low & 25 & 35 & 40 \\
\hline Permanent & High & Low & High & Low & Low & Medium & 15 & 25 & 60 \\
\hline Permanent & High & Low & High & Low & Low & High & 5 & 25 & 70 \\
\hline Permanent & High & Low & High & Low & Medium & Low & 30 & 40 & 30 \\
\hline Permanent & High & Low & High & Low & Medium & Medium & 20 & 30 & 50 \\
\hline Permanent & High & Low & High & Low & Medium & High & 10 & 30 & 60 \\
\hline Permanent & High & Low & High & Low & High & Low & 40 & 50 & 10 \\
\hline Permanent & High & Low & High & Low & High & Medium & 30 & 40 & 30 \\
\hline Permanent & High & Low & High & Low & High & High & 20 & 40 & 40 \\
\hline Permanent & High & Low & High & Medium & Low & Low & 20 & 30 & 50 \\
\hline Permanent & High & Low & High & Medium & Low & Medium & 10 & 20 & 70 \\
\hline Permanent & High & Low & High & Medium & Low & High & 0 & 20 & 80 \\
\hline Permanent & High & Low & High & Medium & Medium & Low & 25 & 35 & 40 \\
\hline Permanent & High & Low & High & Medium & Medium & Medium & 15 & 25 & 60 \\
\hline Permanent & High & Low & High & Medium & Medium & High & 5 & 25 & 70 \\
\hline Permanent & High & Low & High & Medium & High & Low & 35 & 45 & 20 \\
\hline Permanent & High & Low & High & Medium & High & Medium & 25 & 35 & 40 \\
\hline Permanent & High & Low & High & Medium & High & High & 15 & 35 & 50 \\
\hline
\end{tabular}




\begin{tabular}{|c|c|c|c|c|c|c|c|c|c|}
\hline \multicolumn{7}{|c|}{ Inputs } & \multicolumn{3}{|c|}{ Habitat quality } \\
\hline $\begin{array}{c}\text { Water } \\
\text { availability }\end{array}$ & Emergent & Floating & Terrestrial & $\begin{array}{l}\text { Winter } \\
\text { refuge }\end{array}$ & Submerged & $\begin{array}{l}\text { Water } \\
\text { quality }\end{array}$ & Low & Average & High \\
\hline Permanent & High & Low & High & High & Low & Low & 20 & 25 & 55 \\
\hline Permanent & High & Low & High & High & Low & Medium & 10 & 15 & 75 \\
\hline Permanent & High & Low & High & High & Low & High & 0 & 15 & 85 \\
\hline Permanent & High & Low & High & High & Medium & Low & 25 & 30 & 45 \\
\hline Permanent & High & Low & High & High & Medium & Medium & 15 & 20 & 65 \\
\hline Permanent & High & Low & High & High & Medium & High & 5 & 20 & 75 \\
\hline Permanent & High & Low & High & High & High & Low & 35 & 40 & 25 \\
\hline Permanent & High & Low & High & High & High & Medium & 25 & 30 & 45 \\
\hline Permanent & High & Low & High & High & High & High & 15 & 30 & 55 \\
\hline Permanent & High & Medium & Low & Low & Low & Low & 35 & 45 & 20 \\
\hline Permanent & High & Medium & Low & Low & Low & Medium & 25 & 35 & 40 \\
\hline Permanent & High & Medium & Low & Low & Low & High & 15 & 35 & 50 \\
\hline Permanent & High & Medium & Low & Low & Medium & Low & 40 & 50 & 10 \\
\hline Permanent & High & Medium & Low & Low & Medium & Medium & 30 & 40 & 30 \\
\hline Permanent & High & Medium & Low & Low & Medium & High & 20 & 40 & 40 \\
\hline Permanent & High & Medium & Low & Low & High & Low & 50 & 50 & 0 \\
\hline Permanent & High & Medium & Low & Low & High & Medium & 40 & 50 & 10 \\
\hline Permanent & High & Medium & Low & Low & High & High & 30 & 50 & 20 \\
\hline Permanent & High & Medium & Low & Medium & Low & Low & 30 & 40 & 30 \\
\hline Permanent & High & Medium & Low & Medium & Low & Medium & 20 & 30 & 50 \\
\hline Permanent & High & Medium & Low & Medium & Low & High & 10 & 30 & 60 \\
\hline Permanent & High & Medium & Low & Medium & Medium & Low & 35 & 45 & 20 \\
\hline Permanent & High & Medium & Low & Medium & Medium & Medium & 25 & 35 & 40 \\
\hline Permanent & High & Medium & Low & Medium & Medium & High & 15 & 35 & 50 \\
\hline Permanent & High & Medium & Low & Medium & High & Low & 45 & 55 & 0 \\
\hline Permanent & High & Medium & Low & Medium & High & Medium & 35 & 45 & 20 \\
\hline Permanent & High & Medium & Low & Medium & High & High & 25 & 45 & 30 \\
\hline Permanent & High & Medium & Low & High & Low & Low & 30 & 35 & 35 \\
\hline Permanent & High & Medium & Low & High & Low & Medium & 20 & 25 & 55 \\
\hline Permanent & High & Medium & Low & High & Low & High & 10 & 25 & 65 \\
\hline Permanent & High & Medium & Low & High & Medium & Low & 35 & 40 & 25 \\
\hline Permanent & High & Medium & Low & High & Medium & Medium & 25 & 30 & 45 \\
\hline Permanent & High & Medium & Low & High & Medium & High & 15 & 30 & 55 \\
\hline Permanent & High & Medium & Low & High & High & Low & 45 & 50 & 5 \\
\hline Permanent & High & Medium & Low & High & High & Medium & 35 & 40 & 25 \\
\hline Permanent & High & Medium & Low & High & High & High & 25 & 40 & 35 \\
\hline Permanent & High & Medium & Medium & Low & Low & Low & 30 & 40 & 30 \\
\hline Permanent & High & Medium & Medium & Low & Low & Medium & 20 & 30 & 50 \\
\hline Permanent & High & Medium & Medium & Low & Low & High & 10 & 30 & 60 \\
\hline Permanent & High & Medium & Medium & Low & Medium & Low & 35 & 45 & 20 \\
\hline
\end{tabular}




\begin{tabular}{|c|c|c|c|c|c|c|c|c|c|}
\hline \multicolumn{7}{|c|}{ Inputs } & \multicolumn{3}{|c|}{ Habitat quality } \\
\hline $\begin{array}{c}\text { Water } \\
\text { availability }\end{array}$ & Emergent & Floating & Terrestrial & $\begin{array}{l}\text { Winter } \\
\text { refuge }\end{array}$ & Submerged & $\begin{array}{l}\text { Water } \\
\text { quality }\end{array}$ & Low & Average & High \\
\hline Permanent & High & Medium & Medium & Low & Medium & Medium & 25 & 35 & 40 \\
\hline Permanent & High & Medium & Medium & Low & Medium & High & 15 & 35 & 50 \\
\hline Permanent & High & Medium & Medium & Low & High & Low & 45 & 55 & 0 \\
\hline Permanent & High & Medium & Medium & Low & High & Medium & 35 & 45 & 20 \\
\hline Permanent & High & Medium & Medium & Low & High & High & 25 & 45 & 30 \\
\hline Permanent & High & Medium & Medium & Medium & Low & Low & 25 & 35 & 40 \\
\hline Permanent & High & Medium & Medium & Medium & Low & Medium & 15 & 25 & 60 \\
\hline Permanent & High & Medium & Medium & Medium & Low & High & 5 & 25 & 70 \\
\hline Permanent & High & Medium & Medium & Medium & Medium & Low & 30 & 40 & 30 \\
\hline Permanent & High & Medium & Medium & Medium & Medium & Medium & 20 & 30 & 50 \\
\hline Permanent & High & Medium & Medium & Medium & Medium & High & 10 & 30 & 60 \\
\hline Permanent & High & Medium & Medium & Medium & High & Low & 40 & 50 & 10 \\
\hline Permanent & High & Medium & Medium & Medium & High & Medium & 30 & 40 & 30 \\
\hline Permanent & High & Medium & Medium & Medium & High & High & 20 & 40 & 40 \\
\hline Permanent & High & Medium & Medium & High & Low & Low & 25 & 30 & 45 \\
\hline Permanent & High & Medium & Medium & High & Low & Medium & 15 & 20 & 65 \\
\hline Permanent & High & Medium & Medium & High & Low & High & 5 & 20 & 75 \\
\hline Permanent & High & Medium & Medium & High & Medium & Low & 30 & 35 & 35 \\
\hline Permanent & High & Medium & Medium & High & Medium & Medium & 20 & 25 & 55 \\
\hline Permanent & High & Medium & Medium & High & Medium & High & 10 & 25 & 65 \\
\hline Permanent & High & Medium & Medium & High & High & Low & 40 & 45 & 15 \\
\hline Permanent & High & Medium & Medium & High & High & Medium & 30 & 35 & 35 \\
\hline Permanent & High & Medium & Medium & High & High & High & 20 & 35 & 45 \\
\hline Permanent & High & Medium & High & Low & Low & Low & 30 & 35 & 35 \\
\hline Permanent & High & Medium & High & Low & Low & Medium & 20 & 25 & 55 \\
\hline Permanent & High & Medium & High & Low & Low & High & 10 & 25 & 65 \\
\hline Permanent & High & Medium & High & Low & Medium & Low & 35 & 40 & 25 \\
\hline Permanent & High & Medium & High & Low & Medium & Medium & 25 & 30 & 45 \\
\hline Permanent & High & Medium & High & Low & Medium & High & 15 & 30 & 55 \\
\hline Permanent & High & Medium & High & Low & High & Low & 45 & 50 & 5 \\
\hline Permanent & High & Medium & High & Low & High & Medium & 35 & 40 & 25 \\
\hline Permanent & High & Medium & High & Low & High & High & 25 & 40 & 35 \\
\hline Permanent & High & Medium & High & Medium & Low & Low & 25 & 30 & 45 \\
\hline Permanent & High & Medium & High & Medium & Low & Medium & 15 & 20 & 65 \\
\hline Permanent & High & Medium & High & Medium & Low & High & 5 & 20 & 75 \\
\hline Permanent & High & Medium & High & Medium & Medium & Low & 30 & 35 & 35 \\
\hline Permanent & High & Medium & High & Medium & Medium & Medium & 20 & 25 & 55 \\
\hline Permanent & High & Medium & High & Medium & Medium & High & 10 & 25 & 65 \\
\hline Permanent & High & Medium & High & Medium & High & Low & 40 & 45 & 15 \\
\hline Permanent & High & Medium & High & Medium & High & Medium & 30 & 35 & 35 \\
\hline
\end{tabular}




\begin{tabular}{|c|c|c|c|c|c|c|c|c|c|}
\hline \multicolumn{7}{|c|}{ Inputs } & \multicolumn{3}{|c|}{ Habitat quality } \\
\hline $\begin{array}{c}\text { Water } \\
\text { availability }\end{array}$ & Emergent & Floating & Terrestrial & $\begin{array}{l}\text { Winter } \\
\text { refuge }\end{array}$ & Submerged & $\begin{array}{l}\text { Water } \\
\text { quality }\end{array}$ & Low & Average & High \\
\hline Permanent & High & Medium & High & Medium & High & High & 20 & 35 & 45 \\
\hline Permanent & High & Medium & High & High & Low & Low & 25 & 25 & 50 \\
\hline Permanent & High & Medium & High & High & Low & Medium & 15 & 15 & 70 \\
\hline Permanent & High & Medium & High & High & Low & High & 5 & 15 & 80 \\
\hline Permanent & High & Medium & High & High & Medium & Low & 30 & 30 & 40 \\
\hline Permanent & High & Medium & High & High & Medium & Medium & 20 & 20 & 60 \\
\hline Permanent & High & Medium & High & High & Medium & High & 10 & 20 & 70 \\
\hline Permanent & High & Medium & High & High & High & Low & 40 & 40 & 20 \\
\hline Permanent & High & Medium & High & High & High & Medium & 30 & 30 & 40 \\
\hline Permanent & High & Medium & High & High & High & High & 20 & 30 & 50 \\
\hline Permanent & High & High & Low & Low & Low & Low & 40 & 50 & 10 \\
\hline Permanent & High & High & Low & Low & Low & Medium & 30 & 40 & 30 \\
\hline Permanent & High & High & Low & Low & Low & High & 20 & 40 & 40 \\
\hline Permanent & High & High & Low & Low & Medium & Low & 45 & 55 & 0 \\
\hline Permanent & High & High & Low & Low & Medium & Medium & 35 & 45 & 20 \\
\hline Permanent & High & High & Low & Low & Medium & High & 25 & 45 & 30 \\
\hline Permanent & High & High & Low & Low & High & Low & 55 & 45 & 0 \\
\hline Permanent & High & High & Low & Low & High & Medium & 45 & 55 & 0 \\
\hline Permanent & High & High & Low & Low & High & High & 35 & 55 & 10 \\
\hline Permanent & High & High & Low & Medium & Low & Low & 35 & 45 & 20 \\
\hline Permanent & High & High & Low & Medium & Low & Medium & 25 & 35 & 40 \\
\hline Permanent & High & High & Low & Medium & Low & High & 15 & 35 & 50 \\
\hline Permanent & High & High & Low & Medium & Medium & Low & 40 & 50 & 10 \\
\hline Permanent & High & High & Low & Medium & Medium & Medium & 30 & 40 & 30 \\
\hline Permanent & High & High & Low & Medium & Medium & High & 20 & 40 & 40 \\
\hline Permanent & High & High & Low & Medium & High & Low & 50 & 50 & 0 \\
\hline Permanent & High & High & Low & Medium & High & Medium & 40 & 50 & 10 \\
\hline Permanent & High & High & Low & Medium & High & High & 30 & 50 & 20 \\
\hline Permanent & High & High & Low & High & Low & Low & 35 & 40 & 25 \\
\hline Permanent & High & High & Low & High & Low & Medium & 25 & 30 & 45 \\
\hline Permanent & High & High & Low & High & Low & High & 15 & 30 & 55 \\
\hline Permanent & High & High & Low & High & Medium & Low & 40 & 45 & 15 \\
\hline Permanent & High & High & Low & High & Medium & Medium & 30 & 35 & 35 \\
\hline Permanent & High & High & Low & High & Medium & High & 20 & 35 & 45 \\
\hline Permanent & High & High & Low & High & High & Low & 50 & 50 & 0 \\
\hline Permanent & High & High & Low & High & High & Medium & 40 & 45 & 15 \\
\hline Permanent & High & High & Low & High & High & High & 30 & 45 & 25 \\
\hline Permanent & High & High & Medium & Low & Low & Low & 35 & 45 & 20 \\
\hline Permanent & High & High & Medium & Low & Low & Medium & 25 & 35 & 40 \\
\hline Permanent & High & High & Medium & Low & Low & High & 15 & 35 & 50 \\
\hline
\end{tabular}




\begin{tabular}{|c|c|c|c|c|c|c|c|c|c|}
\hline \multicolumn{7}{|c|}{ Inputs } & \multicolumn{3}{|c|}{ Habitat quality } \\
\hline $\begin{array}{c}\text { Water } \\
\text { availability }\end{array}$ & Emergent & Floating & Terrestrial & $\begin{array}{l}\text { Winter } \\
\text { refuge }\end{array}$ & Submerged & $\begin{array}{l}\text { Water } \\
\text { quality }\end{array}$ & Low & Average & High \\
\hline Permanent & High & High & Medium & Low & Medium & Low & 40 & 50 & 10 \\
\hline Permanent & High & High & Medium & Low & Medium & Medium & 30 & 40 & 30 \\
\hline Permanent & High & High & Medium & Low & Medium & High & 20 & 40 & 40 \\
\hline Permanent & High & High & Medium & Low & High & Low & 50 & 50 & 0 \\
\hline Permanent & High & High & Medium & Low & High & Medium & 40 & 50 & 10 \\
\hline Permanent & High & High & Medium & Low & High & High & 30 & 50 & 20 \\
\hline Permanent & High & High & Medium & Medium & Low & Low & 30 & 40 & 30 \\
\hline Permanent & High & High & Medium & Medium & Low & Medium & 20 & 30 & 50 \\
\hline Permanent & High & High & Medium & Medium & Low & High & 10 & 30 & 60 \\
\hline Permanent & High & High & Medium & Medium & Medium & Low & 35 & 45 & 20 \\
\hline Permanent & High & High & Medium & Medium & Medium & Medium & 25 & 35 & 40 \\
\hline Permanent & High & High & Medium & Medium & Medium & High & 15 & 35 & 50 \\
\hline Permanent & High & High & Medium & Medium & High & Low & 45 & 55 & 0 \\
\hline Permanent & High & High & Medium & Medium & High & Medium & 35 & 45 & 20 \\
\hline Permanent & High & High & Medium & Medium & High & High & 25 & 45 & 30 \\
\hline Permanent & High & High & Medium & High & Low & Low & 30 & 35 & 35 \\
\hline Permanent & High & High & Medium & High & Low & Medium & 20 & 25 & 55 \\
\hline Permanent & High & High & Medium & High & Low & High & 10 & 25 & 65 \\
\hline Permanent & High & High & Medium & High & Medium & Low & 35 & 40 & 25 \\
\hline Permanent & High & High & Medium & High & Medium & Medium & 25 & 30 & 45 \\
\hline Permanent & High & High & Medium & High & Medium & High & 15 & 30 & 55 \\
\hline Permanent & High & High & Medium & High & High & Low & 45 & 50 & 5 \\
\hline Permanent & High & High & Medium & High & High & Medium & 35 & 40 & 25 \\
\hline Permanent & High & High & Medium & High & High & High & 25 & 40 & 35 \\
\hline Permanent & High & High & High & Low & Low & Low & 35 & 40 & 25 \\
\hline Permanent & High & High & High & Low & Low & Medium & 25 & 30 & 45 \\
\hline Permanent & High & High & High & Low & Low & High & 15 & 30 & 55 \\
\hline Permanent & High & High & High & Low & Medium & Low & 40 & 45 & 15 \\
\hline Permanent & High & High & High & Low & Medium & Medium & 30 & 35 & 35 \\
\hline Permanent & High & High & High & Low & Medium & High & 20 & 35 & 45 \\
\hline Permanent & High & High & High & Low & High & Low & 50 & 50 & 0 \\
\hline Permanent & High & High & High & Low & High & Medium & 40 & 45 & 15 \\
\hline Permanent & High & High & High & Low & High & High & 30 & 45 & 25 \\
\hline Permanent & High & High & High & Medium & Low & Low & 30 & 35 & 35 \\
\hline Permanent & High & High & High & Medium & Low & Medium & 20 & 25 & 55 \\
\hline Permanent & High & High & High & Medium & Low & High & 10 & 25 & 65 \\
\hline Permanent & High & High & High & Medium & Medium & Low & 35 & 40 & 25 \\
\hline Permanent & High & High & High & Medium & Medium & Medium & 25 & 30 & 45 \\
\hline Permanent & High & High & High & Medium & Medium & High & 15 & 30 & 55 \\
\hline Permanent & High & High & High & Medium & High & Low & 45 & 50 & 5 \\
\hline
\end{tabular}




\begin{tabular}{|c|c|c|c|c|c|c|c|c|c|}
\hline \multicolumn{7}{|c|}{ Inputs } & \multicolumn{3}{|c|}{ Habitat quality } \\
\hline $\begin{array}{c}\text { Water } \\
\text { availability }\end{array}$ & Emergent & Floating & Terrestrial & $\begin{array}{l}\text { Winter } \\
\text { refuge }\end{array}$ & Submerged & $\begin{array}{l}\text { Water } \\
\text { quality }\end{array}$ & Low & Average & High \\
\hline Permanent & High & High & High & Medium & High & Medium & 35 & 40 & 25 \\
\hline Permanent & High & High & High & Medium & High & High & 25 & 40 & 35 \\
\hline Permanent & High & High & High & High & Low & Low & 30 & 30 & 40 \\
\hline Permanent & High & High & High & High & Low & Medium & 20 & 20 & 60 \\
\hline Permanent & High & High & High & High & Low & High & 10 & 20 & 70 \\
\hline Permanent & High & High & High & High & Medium & Low & 35 & 35 & 30 \\
\hline Permanent & High & High & High & High & Medium & Medium & 25 & 25 & 50 \\
\hline Permanent & High & High & High & High & Medium & High & 15 & 25 & 60 \\
\hline Permanent & High & High & High & High & High & Low & 45 & 45 & 10 \\
\hline Permanent & High & High & High & High & High & Medium & 35 & 35 & 30 \\
\hline Permanent & High & High & High & High & High & High & 25 & 35 & 40 \\
\hline
\end{tabular}


Table B4. Conditional probability table (CPT) for the predator, parasite, and pathogen effects node.

[Numbers in the table represent probabilities as percentages (that is, $10=10 \%$ or 0.10 ) that the response node will take the value in the column (low, average, high), given the value of the parent node (habitat quality)]

\begin{tabular}{lllll}
\hline & \multicolumn{4}{c}{ Predator effects } \\
\cline { 2 - 5 } \multicolumn{1}{c}{ Habitat quality } & & Low & Average & High \\
\hline Low & 10 & 40 & 50 \\
Average & 30 & 40 & 30 \\
High & 50 & 40 & 10 \\
\hline
\end{tabular}

Table B5. Conditional probability table (CPT) for the giant gartersnake (Thamnophis gigas) fecundity node.

[Numbers in the table represent probabilities as percentages (that is, $45=45 \%$ or 0.45 ) that the response node will take the value in the column (none, few, average, many), given the value of the parent nodes (prey availability and water quality)]

\begin{tabular}{|c|c|c|c|c|c|}
\hline \multicolumn{2}{|c|}{ Inputs } & \multicolumn{4}{|c|}{ Number of young/Adult female } \\
\hline Prey availability & Water quality & None & Few & Average & Many \\
\hline Low & Low & 45 & 30 & 25 & 0 \\
\hline Low & Medium & 35 & 30 & 35 & 0 \\
\hline Low & High & 30 & 30 & 40 & 0 \\
\hline Medium & Low & 20 & 35 & 40 & 5 \\
\hline Medium & Medium & 15 & 25 & 45 & 15 \\
\hline Medium & High & 10 & 20 & 50 & 20 \\
\hline High & Low & 15 & 25 & 45 & 15 \\
\hline High & Medium & 10 & 15 & 50 & 25 \\
\hline High & High & 5 & 10 & 55 & 30 \\
\hline
\end{tabular}


Table B6. Conditional probability table (CPT) for the giant gartersnake (Thamnophis gigas) first-year survival node.

[Numbers in the table represent probabilities as percentages (that is, $40=40 \%$ or 0.40 ) that the response node will take the value in the column (low, average, high), given the value of the parent nodes (predator, parasite, and pathogen effects; habitat quality; prey availability; water quality; and other sources of mortality)]

\begin{tabular}{|c|c|c|c|c|c|c|c|}
\hline \multicolumn{5}{|c|}{ Inputs } & \multicolumn{3}{|c|}{ First year survival } \\
\hline Predators & $\begin{array}{l}\text { Habitat } \\
\text { quality }\end{array}$ & $\begin{array}{c}\text { Prey } \\
\text { availability }\end{array}$ & $\begin{array}{l}\text { Water } \\
\text { quality }\end{array}$ & $\begin{array}{c}\text { Other } \\
\text { mortality }\end{array}$ & Low & Average & High \\
\hline Low & Low & Low & Low & Low & 40 & 40 & 20 \\
\hline Low & Low & Low & Low & Medium & 40 & 45 & 15 \\
\hline Low & Low & Low & Low & High & 45 & 45 & 10 \\
\hline Low & Low & Low & Medium & Low & 40 & 35 & 25 \\
\hline Low & Low & Low & Medium & Medium & 40 & 40 & 20 \\
\hline Low & Low & Low & Medium & High & 45 & 40 & 15 \\
\hline Low & Low & Low & High & Low & 35 & 35 & 30 \\
\hline Low & Low & Low & High & Medium & 35 & 40 & 25 \\
\hline Low & Low & Low & High & High & 40 & 40 & 20 \\
\hline Low & Low & Average & Low & Low & 35 & 35 & 30 \\
\hline Low & Low & Average & Low & Medium & 35 & 40 & 25 \\
\hline Low & Low & Average & Low & High & 40 & 40 & 20 \\
\hline Low & Low & Average & Medium & Low & 35 & 30 & 35 \\
\hline Low & Low & Average & Medium & Medium & 35 & 35 & 30 \\
\hline Low & Low & Average & Medium & High & 40 & 35 & 25 \\
\hline Low & Low & Average & High & Low & 30 & 30 & 40 \\
\hline Low & Low & Average & High & Medium & 30 & 35 & 35 \\
\hline Low & Low & Average & High & High & 35 & 35 & 30 \\
\hline Low & Low & High & Low & Low & 30 & 30 & 40 \\
\hline Low & Low & High & Low & Medium & 30 & 35 & 35 \\
\hline Low & Low & High & Low & High & 35 & 35 & 30 \\
\hline Low & Low & High & Medium & Low & 30 & 25 & 45 \\
\hline Low & Low & High & Medium & Medium & 30 & 30 & 40 \\
\hline Low & Low & High & Medium & High & 35 & 30 & 35 \\
\hline Low & Low & High & High & Low & 25 & 25 & 50 \\
\hline Low & Low & High & High & Medium & 25 & 30 & 45 \\
\hline Low & Low & High & High & High & 30 & 30 & 40 \\
\hline Low & Average & Low & Low & Low & 20 & 40 & 40 \\
\hline Low & Average & Low & Low & Medium & 20 & 45 & 35 \\
\hline Low & Average & Low & Low & High & 25 & 45 & 30 \\
\hline Low & Average & Low & Medium & Low & 20 & 35 & 45 \\
\hline Low & Average & Low & Medium & Medium & 20 & 40 & 40 \\
\hline Low & Average & Low & Medium & High & 25 & 40 & 35 \\
\hline
\end{tabular}




\begin{tabular}{|c|c|c|c|c|c|c|c|}
\hline \multicolumn{5}{|c|}{ Inputs } & \multicolumn{3}{|c|}{ First year survival } \\
\hline Predators & $\begin{array}{l}\text { Habitat } \\
\text { quality }\end{array}$ & $\begin{array}{c}\text { Prey } \\
\text { availability }\end{array}$ & $\begin{array}{l}\text { Water } \\
\text { quality }\end{array}$ & $\begin{array}{c}\text { Other } \\
\text { mortality }\end{array}$ & Low & Average & High \\
\hline Low & Average & Low & High & Low & 15 & 35 & 50 \\
\hline Low & Average & Low & High & Medium & 15 & 40 & 45 \\
\hline Low & Average & Low & High & High & 20 & 40 & 40 \\
\hline Low & Average & Average & Low & Low & 15 & 35 & 50 \\
\hline Low & Average & Average & Low & Medium & 15 & 40 & 45 \\
\hline Low & Average & Average & Low & High & 20 & 40 & 40 \\
\hline Low & Average & Average & Medium & Low & 15 & 30 & 55 \\
\hline Low & Average & Average & Medium & Medium & 15 & 35 & 50 \\
\hline Low & Average & Average & Medium & High & 20 & 35 & 45 \\
\hline Low & Average & Average & High & Low & 10 & 30 & 60 \\
\hline Low & Average & Average & High & Medium & 10 & 35 & 55 \\
\hline Low & Average & Average & High & High & 15 & 35 & 50 \\
\hline Low & Average & High & Low & Low & 10 & 30 & 60 \\
\hline Low & Average & High & Low & Medium & 10 & 35 & 55 \\
\hline Low & Average & High & Low & High & 15 & 35 & 50 \\
\hline Low & Average & High & Medium & Low & 10 & 25 & 65 \\
\hline Low & Average & High & Medium & Medium & 10 & 30 & 60 \\
\hline Low & Average & High & Medium & High & 15 & 30 & 55 \\
\hline Low & Average & High & High & Low & 5 & 25 & 70 \\
\hline Low & Average & High & High & Medium & 5 & 30 & 65 \\
\hline Low & Average & High & High & High & 10 & 30 & 60 \\
\hline Low & High & Low & Low & Low & 15 & 25 & 60 \\
\hline Low & High & Low & Low & Medium & 15 & 30 & 55 \\
\hline Low & High & Low & Low & High & 20 & 30 & 50 \\
\hline Low & High & Low & Medium & Low & 15 & 20 & 65 \\
\hline Low & High & Low & Medium & Medium & 15 & 25 & 60 \\
\hline Low & High & Low & Medium & High & 20 & 25 & 55 \\
\hline Low & High & Low & High & Low & 10 & 20 & 70 \\
\hline Low & High & Low & High & Medium & 10 & 25 & 65 \\
\hline Low & High & Low & High & High & 15 & 25 & 60 \\
\hline Low & High & Average & Low & Low & 10 & 20 & 70 \\
\hline Low & High & Average & Low & Medium & 10 & 25 & 65 \\
\hline Low & High & Average & Low & High & 15 & 25 & 60 \\
\hline Low & High & Average & Medium & Low & 10 & 15 & 75 \\
\hline Low & High & Average & Medium & Medium & 10 & 20 & 70 \\
\hline Low & High & Average & Medium & High & 15 & 20 & 65 \\
\hline Low & High & Average & High & Low & 5 & 15 & 80 \\
\hline Low & High & Average & High & Medium & 5 & 20 & 75 \\
\hline Low & High & Average & High & High & 10 & 20 & 70 \\
\hline
\end{tabular}




\begin{tabular}{|c|c|c|c|c|c|c|c|}
\hline \multicolumn{5}{|c|}{ Inputs } & \multicolumn{3}{|c|}{ First year survival } \\
\hline Predators & $\begin{array}{l}\text { Habitat } \\
\text { quality }\end{array}$ & $\begin{array}{c}\text { Prey } \\
\text { availability }\end{array}$ & $\begin{array}{l}\text { Water } \\
\text { quality }\end{array}$ & $\begin{array}{c}\text { Other } \\
\text { mortality }\end{array}$ & Low & Average & High \\
\hline Low & High & High & Low & Low & 5 & 15 & 80 \\
\hline Low & High & High & Low & Medium & 5 & 20 & 75 \\
\hline Low & High & High & Low & High & 10 & 20 & 70 \\
\hline Low & High & High & Medium & Low & 5 & 10 & 85 \\
\hline Low & High & High & Medium & Medium & 5 & 15 & 80 \\
\hline Low & High & High & Medium & High & 10 & 15 & 75 \\
\hline Low & High & High & High & Low & 0 & 10 & 90 \\
\hline Low & High & High & High & Medium & 0 & 15 & 85 \\
\hline Low & High & High & High & High & 5 & 15 & 80 \\
\hline Average & Low & Low & Low & Low & 50 & 50 & 0 \\
\hline Average & Low & Low & Low & Medium & 50 & 50 & 0 \\
\hline Average & Low & Low & Low & High & 55 & 45 & 0 \\
\hline Average & Low & Low & Medium & Low & 50 & 45 & 5 \\
\hline Average & Low & Low & Medium & Medium & 50 & 50 & 0 \\
\hline Average & Low & Low & Medium & High & 55 & 45 & 0 \\
\hline Average & Low & Low & High & Low & 45 & 45 & 10 \\
\hline Average & Low & Low & High & Medium & 45 & 50 & 5 \\
\hline Average & Low & Low & High & High & 50 & 50 & 0 \\
\hline Average & Low & Average & Low & Low & 45 & 45 & 10 \\
\hline Average & Low & Average & Low & Medium & 45 & 50 & 5 \\
\hline Average & Low & Average & Low & High & 50 & 50 & 0 \\
\hline Average & Low & Average & Medium & Low & 45 & 40 & 15 \\
\hline Average & Low & Average & Medium & Medium & 45 & 45 & 10 \\
\hline Average & Low & Average & Medium & High & 50 & 45 & 5 \\
\hline Average & Low & Average & High & Low & 40 & 40 & 20 \\
\hline Average & Low & Average & High & Medium & 40 & 45 & 15 \\
\hline Average & Low & Average & High & High & 45 & 45 & 10 \\
\hline Average & Low & High & Low & Low & 40 & 40 & 20 \\
\hline Average & Low & High & Low & Medium & 40 & 45 & 15 \\
\hline Average & Low & High & Low & High & 45 & 45 & 10 \\
\hline Average & Low & High & Medium & Low & 40 & 35 & 25 \\
\hline Average & Low & High & Medium & Medium & 40 & 40 & 20 \\
\hline Average & Low & High & Medium & High & 45 & 40 & 15 \\
\hline Average & Low & High & High & Low & 35 & 35 & 30 \\
\hline Average & Low & High & High & Medium & 35 & 40 & 25 \\
\hline Average & Low & High & High & High & 40 & 40 & 20 \\
\hline Average & Average & Low & Low & Low & 30 & 50 & 20 \\
\hline Average & Average & Low & Low & Medium & 30 & 55 & 15 \\
\hline Average & Average & Low & Low & High & 35 & 55 & 10 \\
\hline
\end{tabular}




\begin{tabular}{|c|c|c|c|c|c|c|c|}
\hline \multicolumn{5}{|c|}{ Inputs } & \multicolumn{3}{|c|}{ First year survival } \\
\hline Predators & $\begin{array}{l}\text { Habitat } \\
\text { quality }\end{array}$ & $\begin{array}{c}\text { Prey } \\
\text { availability }\end{array}$ & $\begin{array}{l}\text { Water } \\
\text { quality }\end{array}$ & $\begin{array}{c}\text { Other } \\
\text { mortality }\end{array}$ & Low & Average & High \\
\hline Average & Average & Low & Medium & Low & 30 & 45 & 25 \\
\hline Average & Average & Low & Medium & Medium & 30 & 50 & 20 \\
\hline Average & Average & Low & Medium & High & 35 & 50 & 15 \\
\hline Average & Average & Low & High & Low & 25 & 45 & 30 \\
\hline Average & Average & Low & High & Medium & 25 & 50 & 25 \\
\hline Average & Average & Low & High & High & 30 & 50 & 20 \\
\hline Average & Average & Average & Low & Low & 25 & 45 & 30 \\
\hline Average & Average & Average & Low & Medium & 25 & 50 & 25 \\
\hline Average & Average & Average & Low & High & 30 & 50 & 20 \\
\hline Average & Average & Average & Medium & Low & 25 & 40 & 35 \\
\hline Average & Average & Average & Medium & Medium & 25 & 45 & 30 \\
\hline Average & Average & Average & Medium & High & 30 & 45 & 25 \\
\hline Average & Average & Average & High & Low & 20 & 40 & 40 \\
\hline Average & Average & Average & High & Medium & 20 & 45 & 35 \\
\hline Average & Average & Average & High & High & 25 & 45 & 30 \\
\hline Average & Average & High & Low & Low & 20 & 40 & 40 \\
\hline Average & Average & High & Low & Medium & 20 & 45 & 35 \\
\hline Average & Average & High & Low & High & 25 & 45 & 30 \\
\hline Average & Average & High & Medium & Low & 20 & 35 & 45 \\
\hline Average & Average & High & Medium & Medium & 20 & 40 & 40 \\
\hline Average & Average & High & Medium & High & 25 & 40 & 35 \\
\hline Average & Average & High & High & Low & 15 & 35 & 50 \\
\hline Average & Average & High & High & Medium & 15 & 40 & 45 \\
\hline Average & Average & High & High & High & 20 & 40 & 40 \\
\hline Average & High & Low & Low & Low & 25 & 35 & 40 \\
\hline Average & High & Low & Low & Medium & 25 & 40 & 35 \\
\hline Average & High & Low & Low & High & 30 & 40 & 30 \\
\hline Average & High & Low & Medium & Low & 25 & 30 & 45 \\
\hline Average & High & Low & Medium & Medium & 25 & 35 & 40 \\
\hline Average & High & Low & Medium & High & 30 & 35 & 35 \\
\hline Average & High & Low & High & Low & 20 & 30 & 50 \\
\hline Average & High & Low & High & Medium & 20 & 35 & 45 \\
\hline Average & High & Low & High & High & 25 & 35 & 40 \\
\hline Average & High & Average & Low & Low & 20 & 30 & 50 \\
\hline Average & High & Average & Low & Medium & 20 & 35 & 45 \\
\hline Average & High & Average & Low & High & 25 & 35 & 40 \\
\hline Average & High & Average & Medium & Low & 20 & 25 & 55 \\
\hline Average & High & Average & Medium & Medium & 20 & 30 & 50 \\
\hline Average & High & Average & Medium & High & 25 & 30 & 45 \\
\hline
\end{tabular}




\begin{tabular}{|c|c|c|c|c|c|c|c|}
\hline \multicolumn{5}{|c|}{ Inputs } & \multicolumn{3}{|c|}{ First year survival } \\
\hline Predators & $\begin{array}{l}\text { Habitat } \\
\text { quality }\end{array}$ & $\begin{array}{c}\text { Prey } \\
\text { availability }\end{array}$ & $\begin{array}{l}\text { Water } \\
\text { quality }\end{array}$ & $\begin{array}{c}\text { Other } \\
\text { mortality }\end{array}$ & Low & Average & High \\
\hline Average & High & Average & High & Low & 15 & 25 & 60 \\
\hline Average & High & Average & High & Medium & 15 & 30 & 55 \\
\hline Average & High & Average & High & High & 20 & 30 & 50 \\
\hline Average & High & High & Low & Low & 15 & 25 & 60 \\
\hline Average & High & High & Low & Medium & 15 & 30 & 55 \\
\hline Average & High & High & Low & High & 20 & 30 & 50 \\
\hline Average & High & High & Medium & Low & 15 & 20 & 65 \\
\hline Average & High & High & Medium & Medium & 15 & 25 & 60 \\
\hline Average & High & High & Medium & High & 20 & 25 & 55 \\
\hline Average & High & High & High & Low & 10 & 20 & 70 \\
\hline Average & High & High & High & Medium & 10 & 25 & 65 \\
\hline Average & High & High & High & High & 15 & 25 & 60 \\
\hline High & Low & Low & Low & Low & 80 & 20 & 0 \\
\hline High & Low & Low & Low & Medium & 80 & 20 & 0 \\
\hline High & Low & Low & Low & High & 85 & 15 & 0 \\
\hline High & Low & Low & Medium & Low & 80 & 20 & 0 \\
\hline High & Low & Low & Medium & Medium & 80 & 20 & 0 \\
\hline High & Low & Low & Medium & High & 85 & 15 & 0 \\
\hline High & Low & Low & High & Low & 75 & 25 & 0 \\
\hline High & Low & Low & High & Medium & 75 & 25 & 0 \\
\hline High & Low & Low & High & High & 80 & 20 & 0 \\
\hline High & Low & Average & Low & Low & 75 & 25 & 0 \\
\hline High & Low & Average & Low & Medium & 75 & 25 & 0 \\
\hline High & Low & Average & Low & High & 80 & 20 & 0 \\
\hline High & Low & Average & Medium & Low & 75 & 25 & 0 \\
\hline High & Low & Average & Medium & Medium & 75 & 25 & 0 \\
\hline High & Low & Average & Medium & High & 80 & 20 & 0 \\
\hline High & Low & Average & High & Low & 70 & 30 & 0 \\
\hline High & Low & Average & High & Medium & 70 & 30 & 0 \\
\hline High & Low & Average & High & High & 75 & 25 & 0 \\
\hline High & Low & High & Low & Low & 70 & 30 & 0 \\
\hline High & Low & High & Low & Medium & 70 & 30 & 0 \\
\hline High & Low & High & Low & High & 75 & 25 & 0 \\
\hline High & Low & High & Medium & Low & 70 & 25 & 5 \\
\hline High & Low & High & Medium & Medium & 70 & 30 & 0 \\
\hline High & Low & High & Medium & High & 75 & 25 & 0 \\
\hline High & Low & High & High & Low & 65 & 25 & 10 \\
\hline High & Low & High & High & Medium & 65 & 30 & 5 \\
\hline High & Low & High & High & High & 70 & 30 & 0 \\
\hline
\end{tabular}




\begin{tabular}{|c|c|c|c|c|c|c|c|}
\hline \multicolumn{5}{|c|}{ Inputs } & \multicolumn{3}{|c|}{ First year survival } \\
\hline Predators & $\begin{array}{l}\text { Habitat } \\
\text { quality }\end{array}$ & $\begin{array}{c}\text { Prey } \\
\text { availability }\end{array}$ & $\begin{array}{l}\text { Water } \\
\text { quality }\end{array}$ & $\begin{array}{c}\text { Other } \\
\text { mortality }\end{array}$ & Low & Average & High \\
\hline High & Average & Low & Low & Low & 60 & 40 & 0 \\
\hline High & Average & Low & Low & Medium & 60 & 40 & 0 \\
\hline High & Average & Low & Low & High & 65 & 35 & 0 \\
\hline High & Average & Low & Medium & Low & 60 & 35 & 5 \\
\hline High & Average & Low & Medium & Medium & 60 & 40 & 0 \\
\hline High & Average & Low & Medium & High & 65 & 35 & 0 \\
\hline High & Average & Low & High & Low & 55 & 35 & 10 \\
\hline High & Average & Low & High & Medium & 55 & 40 & 5 \\
\hline High & Average & Low & High & High & 60 & 40 & 0 \\
\hline High & Average & Average & Low & Low & 55 & 35 & 10 \\
\hline High & Average & Average & Low & Medium & 55 & 40 & 5 \\
\hline High & Average & Average & Low & High & 60 & 40 & 0 \\
\hline High & Average & Average & Medium & Low & 55 & 30 & 15 \\
\hline High & Average & Average & Medium & Medium & 55 & 35 & 10 \\
\hline High & Average & Average & Medium & High & 60 & 35 & 5 \\
\hline High & Average & Average & High & Low & 50 & 30 & 20 \\
\hline High & Average & Average & High & Medium & 50 & 35 & 15 \\
\hline High & Average & Average & High & High & 55 & 35 & 10 \\
\hline High & Average & High & Low & Low & 50 & 30 & 20 \\
\hline High & Average & High & Low & Medium & 50 & 35 & 15 \\
\hline High & Average & High & Low & High & 55 & 35 & 10 \\
\hline High & Average & High & Medium & Low & 50 & 25 & 25 \\
\hline High & Average & High & Medium & Medium & 50 & 30 & 20 \\
\hline High & Average & High & Medium & High & 55 & 30 & 15 \\
\hline High & Average & High & High & Low & 45 & 25 & 30 \\
\hline High & Average & High & High & Medium & 45 & 30 & 25 \\
\hline High & Average & High & High & High & 50 & 30 & 20 \\
\hline High & High & Low & Low & Low & 55 & 25 & 20 \\
\hline High & High & Low & Low & Medium & 55 & 30 & 15 \\
\hline High & High & Low & Low & High & 60 & 30 & 10 \\
\hline High & High & Low & Medium & Low & 55 & 20 & 25 \\
\hline High & High & Low & Medium & Medium & 55 & 25 & 20 \\
\hline High & High & Low & Medium & High & 60 & 25 & 15 \\
\hline High & High & Low & High & Low & 50 & 20 & 30 \\
\hline High & High & Low & High & Medium & 50 & 25 & 25 \\
\hline High & High & Low & High & High & 55 & 25 & 20 \\
\hline High & High & Average & Low & Low & 50 & 20 & 30 \\
\hline High & High & Average & Low & Medium & 50 & 25 & 25 \\
\hline High & High & Average & Low & High & 55 & 25 & 20 \\
\hline
\end{tabular}




\begin{tabular}{|c|c|c|c|c|c|c|c|}
\hline \multicolumn{5}{|c|}{ Inputs } & \multicolumn{3}{|c|}{ First year survival } \\
\hline Predators & $\begin{array}{l}\text { Habitat } \\
\text { quality }\end{array}$ & $\begin{array}{c}\text { Prey } \\
\text { availability }\end{array}$ & $\begin{array}{l}\text { Water } \\
\text { quality }\end{array}$ & $\begin{array}{c}\text { Other } \\
\text { mortality }\end{array}$ & Low & Average & High \\
\hline High & High & Average & Medium & Low & 50 & 15 & 35 \\
\hline High & High & Average & Medium & Medium & 50 & 20 & 30 \\
\hline High & High & Average & Medium & High & 55 & 20 & 25 \\
\hline High & High & Average & High & Low & 45 & 15 & 40 \\
\hline High & High & Average & High & Medium & 45 & 20 & 35 \\
\hline High & High & Average & High & High & 50 & 20 & 30 \\
\hline High & High & High & Low & Low & 45 & 15 & 40 \\
\hline High & High & High & Low & Medium & 45 & 20 & 35 \\
\hline High & High & High & Low & High & 50 & 20 & 30 \\
\hline High & High & High & Medium & Low & 45 & 10 & 45 \\
\hline High & High & High & Medium & Medium & 45 & 15 & 40 \\
\hline High & High & High & Medium & High & 50 & 15 & 35 \\
\hline High & High & High & High & Low & 40 & 10 & 50 \\
\hline High & High & High & High & Medium & 40 & 15 & 45 \\
\hline High & High & High & High & High & 45 & 15 & 40 \\
\hline
\end{tabular}


Table B7. Conditional probability table (CPT) for the giant gartersnake (Thamnophis gigas) adult survival node.

[Numbers in the table represent probabilities as percentages (that is, $30=30 \%$ or 0.30 ) that the response node will take the value in the column (low, average, high), given the value of the parent nodes (predator, parasite, and pathogen effects; habitat quality; prey availability; water quality; and other sources of mortality)]

\begin{tabular}{|c|c|c|c|c|c|c|c|}
\hline \multicolumn{5}{|c|}{ Inputs } & \multicolumn{3}{|c|}{ Adult Survival } \\
\hline $\begin{array}{c}\text { Prey } \\
\text { availability }\end{array}$ & $\begin{array}{c}\text { Predator } \\
\text { effects }\end{array}$ & $\begin{array}{l}\text { Habitat } \\
\text { quality }\end{array}$ & $\begin{array}{l}\text { Water } \\
\text { quality }\end{array}$ & $\begin{array}{c}\text { Other } \\
\text { mortality }\end{array}$ & Low & Average & High \\
\hline Low & Low & Low & Low & Low & 30 & 40 & 30 \\
\hline Low & Low & Low & Low & Medium & 30 & 45 & 25 \\
\hline Low & Low & Low & Low & High & 35 & 45 & 20 \\
\hline Low & Low & Low & Medium & Low & 25 & 40 & 35 \\
\hline Low & Low & Low & Medium & Medium & 25 & 45 & 30 \\
\hline Low & Low & Low & Medium & High & 30 & 45 & 25 \\
\hline Low & Low & Low & High & Low & 25 & 35 & 40 \\
\hline Low & Low & Low & High & Medium & 25 & 40 & 35 \\
\hline Low & Low & Low & High & High & 30 & 40 & 30 \\
\hline Low & Low & Average & Low & Low & 15 & 35 & 50 \\
\hline Low & Low & Average & Low & Medium & 15 & 40 & 45 \\
\hline Low & Low & Average & Low & High & 20 & 40 & 40 \\
\hline Low & Low & Average & Medium & Low & 10 & 35 & 55 \\
\hline Low & Low & Average & Medium & Medium & 10 & 40 & 50 \\
\hline Low & Low & Average & Medium & High & 15 & 40 & 45 \\
\hline Low & Low & Average & High & Low & 10 & 30 & 60 \\
\hline Low & Low & Average & High & Medium & 10 & 35 & 55 \\
\hline Low & Low & Average & High & High & 15 & 35 & 50 \\
\hline Low & Low & High & Low & Low & 10 & 20 & 70 \\
\hline Low & Low & High & Low & Medium & 10 & 25 & 65 \\
\hline Low & Low & High & Low & High & 15 & 25 & 60 \\
\hline Low & Low & High & Medium & Low & 5 & 20 & 75 \\
\hline Low & Low & High & Medium & Medium & 5 & 25 & 70 \\
\hline Low & Low & High & Medium & High & 10 & 25 & 65 \\
\hline Low & Low & High & High & Low & 5 & 15 & 80 \\
\hline Low & Low & High & High & Medium & 5 & 20 & 75 \\
\hline Low & Low & High & High & High & 10 & 20 & 70 \\
\hline Low & Average & Low & Low & Low & 35 & 50 & 15 \\
\hline Low & Average & Low & Low & Medium & 35 & 55 & 10 \\
\hline Low & Average & Low & Low & High & 40 & 55 & 5 \\
\hline Low & Average & Low & Medium & Low & 30 & 50 & 20 \\
\hline Low & Average & Low & Medium & Medium & 30 & 55 & 15 \\
\hline Low & Average & Low & Medium & High & 35 & 55 & 10 \\
\hline Low & Average & Low & High & Low & 30 & 45 & 25 \\
\hline
\end{tabular}




\begin{tabular}{|c|c|c|c|c|c|c|c|}
\hline \multicolumn{5}{|c|}{ Inputs } & \multicolumn{3}{|c|}{ Adult Survival } \\
\hline $\begin{array}{c}\text { Prey } \\
\text { availability }\end{array}$ & $\begin{array}{c}\text { Predator } \\
\text { effects }\end{array}$ & $\begin{array}{l}\text { Habitat } \\
\text { quality }\end{array}$ & $\begin{array}{l}\text { Water } \\
\text { quality }\end{array}$ & $\begin{array}{c}\text { Other } \\
\text { mortality }\end{array}$ & Low & Average & High \\
\hline Low & Average & Low & High & Medium & 30 & 50 & 20 \\
\hline Low & Average & Low & High & High & 35 & 50 & 15 \\
\hline Low & Average & Average & Low & Low & 20 & 45 & 35 \\
\hline Low & Average & Average & Low & Medium & 20 & 50 & 30 \\
\hline Low & Average & Average & Low & High & 25 & 50 & 25 \\
\hline Low & Average & Average & Medium & Low & 15 & 45 & 40 \\
\hline Low & Average & Average & Medium & Medium & 15 & 50 & 35 \\
\hline Low & Average & Average & Medium & High & 20 & 50 & 30 \\
\hline Low & Average & Average & High & Low & 15 & 40 & 45 \\
\hline Low & Average & Average & High & Medium & 15 & 45 & 40 \\
\hline Low & Average & Average & High & High & 20 & 45 & 35 \\
\hline Low & Average & High & Low & Low & 15 & 30 & 55 \\
\hline Low & Average & High & Low & Medium & 15 & 35 & 50 \\
\hline Low & Average & High & Low & High & 20 & 35 & 45 \\
\hline Low & Average & High & Medium & Low & 10 & 30 & 60 \\
\hline Low & Average & High & Medium & Medium & 10 & 35 & 55 \\
\hline Low & Average & High & Medium & High & 15 & 35 & 50 \\
\hline Low & Average & High & High & Low & 10 & 25 & 65 \\
\hline Low & Average & High & High & Medium & 10 & 30 & 60 \\
\hline Low & Average & High & High & High & 15 & 30 & 55 \\
\hline Low & High & Low & Low & Low & 50 & 50 & 0 \\
\hline Low & High & Low & Low & Medium & 45 & 55 & 0 \\
\hline Low & High & Low & Low & High & 45 & 55 & 0 \\
\hline Low & High & Low & Medium & Low & 45 & 50 & 5 \\
\hline Low & High & Low & Medium & Medium & 45 & 55 & 0 \\
\hline Low & High & Low & Medium & High & 45 & 55 & 0 \\
\hline Low & High & Low & High & Low & 45 & 45 & 10 \\
\hline Low & High & Low & High & Medium & 45 & 50 & 5 \\
\hline Low & High & Low & High & High & 50 & 50 & 0 \\
\hline Low & High & Average & Low & Low & 35 & 45 & 20 \\
\hline Low & High & Average & Low & Medium & 35 & 50 & 15 \\
\hline Low & High & Average & Low & High & 40 & 50 & 10 \\
\hline Low & High & Average & Medium & Low & 30 & 45 & 25 \\
\hline Low & High & Average & Medium & Medium & 30 & 50 & 20 \\
\hline Low & High & Average & Medium & High & 35 & 50 & 15 \\
\hline Low & High & Average & High & Low & 30 & 40 & 30 \\
\hline Low & High & Average & High & Medium & 30 & 45 & 25 \\
\hline Low & High & Average & High & High & 35 & 45 & 20 \\
\hline Low & High & High & Low & Low & 30 & 30 & 40 \\
\hline
\end{tabular}




\begin{tabular}{|c|c|c|c|c|c|c|c|}
\hline \multicolumn{5}{|c|}{ Inputs } & \multicolumn{3}{|c|}{ Adult Survival } \\
\hline $\begin{array}{c}\text { Prey } \\
\text { availability }\end{array}$ & $\begin{array}{c}\text { Predator } \\
\text { effects }\end{array}$ & $\begin{array}{l}\text { Habitat } \\
\text { quality }\end{array}$ & $\begin{array}{l}\text { Water } \\
\text { quality }\end{array}$ & $\begin{array}{c}\text { Other } \\
\text { mortality }\end{array}$ & Low & Average & High \\
\hline Low & High & High & Low & Medium & 30 & 35 & 35 \\
\hline Low & High & High & Low & High & 35 & 35 & 30 \\
\hline Low & High & High & Medium & Low & 25 & 30 & 45 \\
\hline Low & High & High & Medium & Medium & 25 & 35 & 40 \\
\hline Low & High & High & Medium & High & 30 & 35 & 35 \\
\hline Low & High & High & High & Low & 25 & 25 & 50 \\
\hline Low & High & High & High & Medium & 25 & 30 & 45 \\
\hline Low & High & High & High & High & 30 & 30 & 40 \\
\hline Average & Low & Low & Low & Low & 30 & 35 & 35 \\
\hline Average & Low & Low & Low & Medium & 30 & 40 & 30 \\
\hline Average & Low & Low & Low & High & 35 & 40 & 25 \\
\hline Average & Low & Low & Medium & Low & 25 & 35 & 40 \\
\hline Average & Low & Low & Medium & Medium & 25 & 40 & 35 \\
\hline Average & Low & Low & Medium & High & 30 & 40 & 30 \\
\hline Average & Low & Low & High & Low & 25 & 30 & 45 \\
\hline Average & Low & Low & High & Medium & 25 & 35 & 40 \\
\hline Average & Low & Low & High & High & 30 & 35 & 35 \\
\hline Average & Low & Average & Low & Low & 15 & 30 & 55 \\
\hline Average & Low & Average & Low & Medium & 15 & 35 & 50 \\
\hline Average & Low & Average & Low & High & 20 & 35 & 45 \\
\hline Average & Low & Average & Medium & Low & 10 & 30 & 60 \\
\hline Average & Low & Average & Medium & Medium & 10 & 35 & 55 \\
\hline Average & Low & Average & Medium & High & 15 & 35 & 50 \\
\hline Average & Low & Average & High & Low & 10 & 25 & 65 \\
\hline Average & Low & Average & High & Medium & 10 & 30 & 60 \\
\hline Average & Low & Average & High & High & 15 & 30 & 55 \\
\hline Average & Low & High & Low & Low & 10 & 15 & 75 \\
\hline Average & Low & High & Low & Medium & 10 & 20 & 70 \\
\hline Average & Low & High & Low & High & 15 & 20 & 65 \\
\hline Average & Low & High & Medium & Low & 5 & 15 & 80 \\
\hline Average & Low & High & Medium & Medium & 5 & 20 & 75 \\
\hline Average & Low & High & Medium & High & 10 & 20 & 70 \\
\hline Average & Low & High & High & Low & 5 & 10 & 85 \\
\hline Average & Low & High & High & Medium & 5 & 15 & 80 \\
\hline Average & Low & High & High & High & 10 & 15 & 75 \\
\hline Average & Average & Low & Low & Low & 35 & 45 & 20 \\
\hline Average & Average & Low & Low & Medium & 35 & 50 & 15 \\
\hline Average & Average & Low & Low & High & 40 & 50 & 10 \\
\hline Average & Average & Low & Medium & Low & 30 & 45 & 25 \\
\hline
\end{tabular}




\begin{tabular}{|c|c|c|c|c|c|c|c|}
\hline \multicolumn{5}{|c|}{ Inputs } & \multicolumn{3}{|c|}{ Adult Survival } \\
\hline $\begin{array}{c}\text { Prey } \\
\text { availability }\end{array}$ & $\begin{array}{c}\text { Predator } \\
\text { effects }\end{array}$ & $\begin{array}{l}\text { Habitat } \\
\text { quality }\end{array}$ & $\begin{array}{l}\text { Water } \\
\text { quality }\end{array}$ & $\begin{array}{c}\text { Other } \\
\text { mortality }\end{array}$ & Low & Average & High \\
\hline Average & Average & Low & Medium & Medium & 30 & 50 & 20 \\
\hline Average & Average & Low & Medium & High & 35 & 50 & 15 \\
\hline Average & Average & Low & High & Low & 30 & 40 & 30 \\
\hline Average & Average & Low & High & Medium & 30 & 45 & 25 \\
\hline Average & Average & Low & High & High & 35 & 45 & 20 \\
\hline Average & Average & Average & Low & Low & 20 & 40 & 40 \\
\hline Average & Average & Average & Low & Medium & 20 & 45 & 35 \\
\hline Average & Average & Average & Low & High & 25 & 45 & 30 \\
\hline Average & Average & Average & Medium & Low & 15 & 40 & 45 \\
\hline Average & Average & Average & Medium & Medium & 15 & 45 & 40 \\
\hline Average & Average & Average & Medium & High & 20 & 45 & 35 \\
\hline Average & Average & Average & High & Low & 15 & 35 & 50 \\
\hline Average & Average & Average & High & Medium & 15 & 40 & 45 \\
\hline Average & Average & Average & High & High & 20 & 40 & 40 \\
\hline Average & Average & High & Low & Low & 15 & 25 & 60 \\
\hline Average & Average & High & Low & Medium & 15 & 30 & 55 \\
\hline Average & Average & High & Low & High & 20 & 30 & 50 \\
\hline Average & Average & High & Medium & Low & 10 & 25 & 65 \\
\hline Average & Average & High & Medium & Medium & 10 & 30 & 60 \\
\hline Average & Average & High & Medium & High & 15 & 30 & 55 \\
\hline Average & Average & High & High & Low & 10 & 20 & 70 \\
\hline Average & Average & High & High & Medium & 10 & 25 & 65 \\
\hline Average & Average & High & High & High & 15 & 25 & 60 \\
\hline Average & High & Low & Low & Low & 50 & 45 & 5 \\
\hline Average & High & Low & Low & Medium & 50 & 50 & 0 \\
\hline Average & High & Low & Low & High & 50 & 50 & 0 \\
\hline Average & High & Low & Medium & Low & 45 & 45 & 10 \\
\hline Average & High & Low & Medium & Medium & 45 & 50 & 5 \\
\hline Average & High & Low & Medium & High & 50 & 50 & 0 \\
\hline Average & High & Low & High & Low & 45 & 40 & 15 \\
\hline Average & High & Low & High & Medium & 45 & 45 & 10 \\
\hline Average & High & Low & High & High & 50 & 45 & 5 \\
\hline Average & High & Average & Low & Low & 35 & 40 & 25 \\
\hline Average & High & Average & Low & Medium & 35 & 45 & 20 \\
\hline Average & High & Average & Low & High & 40 & 45 & 15 \\
\hline Average & High & Average & Medium & Low & 30 & 40 & 30 \\
\hline Average & High & Average & Medium & Medium & 30 & 45 & 25 \\
\hline Average & High & Average & Medium & High & 35 & 45 & 20 \\
\hline Average & High & Average & High & Low & 30 & 35 & 35 \\
\hline
\end{tabular}




\begin{tabular}{|c|c|c|c|c|c|c|c|}
\hline \multicolumn{5}{|c|}{ Inputs } & \multicolumn{3}{|c|}{ Adult Survival } \\
\hline $\begin{array}{c}\text { Prey } \\
\text { availability }\end{array}$ & $\begin{array}{c}\text { Predator } \\
\text { effects }\end{array}$ & $\begin{array}{l}\text { Habitat } \\
\text { quality }\end{array}$ & $\begin{array}{l}\text { Water } \\
\text { quality }\end{array}$ & $\begin{array}{c}\text { Other } \\
\text { mortality }\end{array}$ & Low & Average & High \\
\hline Average & High & Average & High & Medium & 30 & 40 & 30 \\
\hline Average & High & Average & High & High & 35 & 40 & 25 \\
\hline Average & High & High & Low & Low & 30 & 25 & 45 \\
\hline Average & High & High & Low & Medium & 30 & 30 & 40 \\
\hline Average & High & High & Low & High & 35 & 30 & 35 \\
\hline Average & High & High & Medium & Low & 25 & 25 & 50 \\
\hline Average & High & High & Medium & Medium & 25 & 30 & 45 \\
\hline Average & High & High & Medium & High & 30 & 30 & 40 \\
\hline Average & High & High & High & Low & 25 & 20 & 55 \\
\hline Average & High & High & High & Medium & 25 & 25 & 50 \\
\hline Average & High & High & High & High & 30 & 25 & 45 \\
\hline High & Low & Low & Low & Low & 25 & 35 & 40 \\
\hline High & Low & Low & Low & Medium & 25 & 40 & 35 \\
\hline High & Low & Low & Low & High & 30 & 40 & 30 \\
\hline High & Low & Low & Medium & Low & 20 & 35 & 45 \\
\hline High & Low & Low & Medium & Medium & 20 & 40 & 40 \\
\hline High & Low & Low & Medium & High & 25 & 40 & 35 \\
\hline High & Low & Low & High & Low & 20 & 30 & 50 \\
\hline High & Low & Low & High & Medium & 20 & 35 & 45 \\
\hline High & Low & Low & High & High & 25 & 35 & 40 \\
\hline High & Low & Average & Low & Low & 10 & 30 & 60 \\
\hline High & Low & Average & Low & Medium & 10 & 35 & 55 \\
\hline High & Low & Average & Low & High & 15 & 35 & 50 \\
\hline High & Low & Average & Medium & Low & 5 & 30 & 65 \\
\hline High & Low & Average & Medium & Medium & 5 & 35 & 60 \\
\hline High & Low & Average & Medium & High & 10 & 35 & 55 \\
\hline High & Low & Average & High & Low & 5 & 25 & 70 \\
\hline High & Low & Average & High & Medium & 5 & 30 & 65 \\
\hline High & Low & Average & High & High & 10 & 30 & 60 \\
\hline High & Low & High & Low & Low & 5 & 15 & 80 \\
\hline High & Low & High & Low & Medium & 5 & 20 & 75 \\
\hline High & Low & High & Low & High & 10 & 20 & 70 \\
\hline High & Low & High & Medium & Low & 0 & 15 & 85 \\
\hline High & Low & High & Medium & Medium & 0 & 20 & 80 \\
\hline High & Low & High & Medium & High & 5 & 20 & 75 \\
\hline High & Low & High & High & Low & 0 & 10 & 90 \\
\hline High & Low & High & High & Medium & 0 & 15 & 85 \\
\hline High & Low & High & High & High & 5 & 15 & 80 \\
\hline High & Average & Low & Low & Low & 30 & 45 & 25 \\
\hline
\end{tabular}




\begin{tabular}{|c|c|c|c|c|c|c|c|}
\hline \multicolumn{5}{|c|}{ Inputs } & \multicolumn{3}{|c|}{ Adult Survival } \\
\hline $\begin{array}{c}\text { Prey } \\
\text { availability }\end{array}$ & $\begin{array}{c}\text { Predator } \\
\text { effects }\end{array}$ & $\begin{array}{l}\text { Habitat } \\
\text { quality }\end{array}$ & $\begin{array}{l}\text { Water } \\
\text { quality }\end{array}$ & $\begin{array}{c}\text { Other } \\
\text { mortality }\end{array}$ & Low & Average & High \\
\hline High & Average & Low & Low & Medium & 30 & 50 & 20 \\
\hline High & Average & Low & Low & High & 35 & 50 & 15 \\
\hline High & Average & Low & Medium & Low & 25 & 45 & 30 \\
\hline High & Average & Low & Medium & Medium & 25 & 50 & 25 \\
\hline High & Average & Low & Medium & High & 30 & 50 & 20 \\
\hline High & Average & Low & High & Low & 25 & 40 & 35 \\
\hline High & Average & Low & High & Medium & 25 & 45 & 30 \\
\hline High & Average & Low & High & High & 30 & 45 & 25 \\
\hline High & Average & Average & Low & Low & 15 & 40 & 45 \\
\hline High & Average & Average & Low & Medium & 15 & 45 & 40 \\
\hline High & Average & Average & Low & High & 20 & 45 & 35 \\
\hline High & Average & Average & Medium & Low & 10 & 40 & 50 \\
\hline High & Average & Average & Medium & Medium & 10 & 45 & 45 \\
\hline High & Average & Average & Medium & High & 15 & 45 & 40 \\
\hline High & Average & Average & High & Low & 10 & 35 & 55 \\
\hline High & Average & Average & High & Medium & 10 & 40 & 50 \\
\hline High & Average & Average & High & High & 15 & 40 & 45 \\
\hline High & Average & High & Low & Low & 10 & 25 & 65 \\
\hline High & Average & High & Low & Medium & 10 & 30 & 60 \\
\hline High & Average & High & Low & High & 15 & 30 & 55 \\
\hline High & Average & High & Medium & Low & 5 & 25 & 70 \\
\hline High & Average & High & Medium & Medium & 5 & 30 & 65 \\
\hline High & Average & High & Medium & High & 10 & 30 & 60 \\
\hline High & Average & High & High & Low & 5 & 20 & 75 \\
\hline High & Average & High & High & Medium & 5 & 25 & 70 \\
\hline High & Average & High & High & High & 10 & 25 & 65 \\
\hline High & High & Low & Low & Low & 45 & 45 & 10 \\
\hline High & High & Low & Low & Medium & 45 & 50 & 5 \\
\hline High & High & Low & Low & High & 50 & 50 & 0 \\
\hline High & High & Low & Medium & Low & 40 & 45 & 15 \\
\hline High & High & Low & Medium & Medium & 40 & 50 & 10 \\
\hline High & High & Low & Medium & High & 45 & 50 & 5 \\
\hline High & High & Low & High & Low & 40 & 40 & 20 \\
\hline High & High & Low & High & Medium & 40 & 45 & 15 \\
\hline High & High & Low & High & High & 45 & 45 & 10 \\
\hline High & High & Average & Low & Low & 30 & 40 & 30 \\
\hline High & High & Average & Low & Medium & 30 & 45 & 25 \\
\hline High & High & Average & Low & High & 35 & 45 & 20 \\
\hline High & High & Average & Medium & Low & 25 & 40 & 35 \\
\hline
\end{tabular}




\begin{tabular}{|c|c|c|c|c|c|c|c|}
\hline \multicolumn{5}{|c|}{ Inputs } & \multicolumn{3}{|c|}{ Adult Survival } \\
\hline $\begin{array}{c}\text { Prey } \\
\text { availability }\end{array}$ & $\begin{array}{c}\text { Predator } \\
\text { effects }\end{array}$ & $\begin{array}{l}\text { Habitat } \\
\text { quality }\end{array}$ & $\begin{array}{l}\text { Water } \\
\text { quality }\end{array}$ & $\begin{array}{c}\text { Other } \\
\text { mortality }\end{array}$ & Low & Average & High \\
\hline High & High & Average & Medium & Medium & 25 & 45 & 30 \\
\hline High & High & Average & Medium & High & 30 & 45 & 25 \\
\hline High & High & Average & High & Low & 25 & 35 & 40 \\
\hline High & High & Average & High & Medium & 25 & 40 & 35 \\
\hline High & High & Average & High & High & 30 & 40 & 30 \\
\hline High & High & High & Low & Low & 25 & 25 & 50 \\
\hline High & High & High & Low & Medium & 25 & 30 & 45 \\
\hline High & High & High & Low & High & 30 & 30 & 40 \\
\hline High & High & High & Medium & Low & 20 & 25 & 55 \\
\hline High & High & High & Medium & Medium & 20 & 30 & 50 \\
\hline High & High & High & Medium & High & 25 & 30 & 45 \\
\hline High & High & High & High & Low & 20 & 20 & 60 \\
\hline High & High & High & High & Medium & 20 & 25 & 55 \\
\hline High & High & High & High & High & 25 & 25 & 50 \\
\hline
\end{tabular}


Table B8. Conditional probability table (CPT) for the giant gartersnake (Thamnophis gigas) population growth node.

[Numbers in the table represent probabilities as percentages (that is, $45=45 \%$ or 0.45 ) that the response node will take the value in the column (none, few, average, many), given the value of the parent nodes (prey availability and water quality)]

\begin{tabular}{|c|c|c|c|c|c|}
\hline \multicolumn{3}{|c|}{ Inputs } & \multicolumn{3}{|c|}{ Population growth rate } \\
\hline Adult survival & Juvenile survival & Fecundity & Decreasing & Stable & Increasing \\
\hline Low & Low & None & 100 & 0 & 0 \\
\hline Low & Low & Few & 90 & 10 & 0 \\
\hline Low & Low & Average & 80 & 20 & 0 \\
\hline Low & Low & Many & 70 & 20 & 10 \\
\hline Low & Average & None & 95 & 5 & 0 \\
\hline Low & Average & Few & 85 & 15 & 0 \\
\hline Low & Average & Average & 75 & 20 & 5 \\
\hline Low & Average & Many & 60 & 25 & 15 \\
\hline Low & High & None & 90 & 10 & 0 \\
\hline Low & High & Few & 80 & 20 & 0 \\
\hline Low & High & Average & 70 & 20 & 10 \\
\hline Low & High & Many & 60 & 25 & 15 \\
\hline Medium & Low & None & 80 & 20 & 0 \\
\hline Medium & Low & Few & 70 & 30 & 0 \\
\hline Medium & Low & Average & 30 & 50 & 20 \\
\hline Medium & Low & Many & 25 & 50 & 25 \\
\hline Medium & Average & None & 60 & 40 & 0 \\
\hline Medium & Average & Few & 40 & 50 & 10 \\
\hline Medium & Average & Average & 25 & 50 & 25 \\
\hline Medium & Average & Many & 15 & 50 & 35 \\
\hline Medium & High & None & 70 & 30 & 0 \\
\hline Medium & High & Few & 50 & 40 & 10 \\
\hline Medium & High & Average & 20 & 50 & 30 \\
\hline Medium & High & Many & 0 & 60 & 40 \\
\hline High & Low & None & 70 & 30 & 0 \\
\hline High & Low & Few & 40 & 40 & 20 \\
\hline High & Low & Average & 20 & 40 & 40 \\
\hline High & Low & Many & 0 & 20 & 80 \\
\hline High & Average & None & 70 & 30 & 0 \\
\hline High & Average & Few & 20 & 40 & 40 \\
\hline High & Average & Average & 10 & 20 & 70 \\
\hline High & Average & Many & 0 & 10 & 90 \\
\hline High & High & None & 60 & 40 & 0 \\
\hline High & High & Few & 10 & 30 & 60 \\
\hline High & High & Average & 0 & 20 & 80 \\
\hline High & High & Many & 0 & 0 & 100 \\
\hline
\end{tabular}


Publishing support provided by the U.S. Geological Survey

Science Publishing Network, Tacoma Publishing Service Center

For more information concerning the research in this report, contact the Director, Western Ecological Research Center

U.S. Geological Survey

3020 State University Drive East

Sacramento, California 95819

http://werc.usgs.gov/ 
